\title{
Diffusion weighted magnetic resonance imaging : validation, correction and applications
}

Citation for published version (APA):

Pullens, W. L. (2012). Diffusion weighted magnetic resonance imaging : validation, correction and applications. [Doctoral Thesis, Maastricht University]. Maastricht University. https://doi.org/10.26481/dis.20120706wp

Document status and date:

Published: 01/01/2012

DOI:

10.26481/dis.20120706wp

Document Version:

Publisher's PDF, also known as Version of record

\section{Please check the document version of this publication:}

- A submitted manuscript is the version of the article upon submission and before peer-review. There can be important differences between the submitted version and the official published version of record. People interested in the research are advised to contact the author for the final version of the publication, or visit the DOI to the publisher's website.

- The final author version and the galley proof are versions of the publication after peer review.

- The final published version features the final layout of the paper including the volume, issue and page numbers.

Link to publication

\footnotetext{
General rights rights.

- You may freely distribute the URL identifying the publication in the public portal. please follow below link for the End User Agreement:

www.umlib.nl/taverne-license

Take down policy

If you believe that this document breaches copyright please contact us at:

repository@maastrichtuniversity.nl

providing details and we will investigate your claim.
}

Copyright and moral rights for the publications made accessible in the public portal are retained by the authors and/or other copyright owners and it is a condition of accessing publications that users recognise and abide by the legal requirements associated with these

- Users may download and print one copy of any publication from the public portal for the purpose of private study or research.

- You may not further distribute the material or use it for any profit-making activity or commercial gain

If the publication is distributed under the terms of Article $25 \mathrm{fa}$ of the Dutch Copyright Act, indicated by the "Taverne" license above, 
DIFFUSION WEIGHTED MAGNETIC RESONANCE IMAGING

VALIDATION, CORRECTION AND APPLICATIONS

PIM PULLENS 
Pim Pullens Diffusion Weighted Magnetic Resonance Imaging: Validation, Correction and Applications (C) May, 2012

ISBN: 978-90-5335-566-4

\section{COLOPHON}

This document was typeset by the author using the typographical look-and-feel classicthesis in LATEX developed by André Miede www. miede. de.

Cover design by the author. Yanone Kaffeesatz font by Yanone www. yanone. de.

Printed by Ridderprint, Ridderkerk, NL 
DIFFUSION WEIGHTED MAGNETIC RESONANCE IMAGING VALIDATION, CORRECTION AND APPLICATIONS

\section{Proefschrift}

Ter verkrijging van de graad van doctor aan de Universiteit Maastricht, op gezag van de Rector Magnificus, Prof. mr. G.P.M.F. Mols volgens het besluit van het College van Decanen, in het openbaar te verdedigen op vrijdag 6 juli 2012 om 16.00 uur

door

Wilhelmus lambertus PETRUS MARIA PULlens

geboren op 19 mei 1979 te 's-Hertogenbosch 
Promotor:

Prof. dr. Rainer Goebel

Copromotor:

Dr. Alard Roebroeck

Reading committee:

Prof. dr. Elia Formisano (chair)

Dr. Alexander Leemans (University of Utrecht)

Prof. dr. Klaas Nicolay (Eindhoven University of Technology)

Prof. dr. N. Jon Shah (Forschungszentrum Jülich, Germany)

Prof. dr. Stefan Sunaert (Katholieke Universiteit Leuven, Belgium) 


\section{CONTENTS}

I PROBING STRUCTURE IN THE BRAIN WITH DIFFUSION MRI

I VALIDATION \& CORRECTION OF DIFFUSION MRI ACQUISITION 19

2 HARDWARE PHANTOMS FOR DWI 21

3 PHANTOM ORIENTATION AFFECTS DIFFUSION MEASURES 37

4 EDDY CURRENT AND MOTION CORRECTION FOR DW-MRI 57

II APPLICATIONS OF DW-MRI $\quad 75$

5 FMRI OF LETTER-SOUND INTEGRATION IN LKS 77

6 LANGUAGE PATHWAYS IN LKS 97

7 SUB-CORTICAL CONNECTIVITY FOLLOWING VISUAL CORTEX DE$\begin{array}{ll}\text { STRUCTION } & 115\end{array}$

8 GENERAL DISCUSSION 135

$\begin{array}{lr}\text { SUMMARY } & 145\end{array}$

$\begin{array}{lr}\text { SAMENVATTING } & 148\end{array}$

$\begin{array}{lr}\text { DANKWOORD } & 151\end{array}$

$\begin{array}{lr}\text { CURRICULUM VITAE } & 154\end{array}$

$\begin{array}{lr}\text { LIST OF PUBLICATIONS } & 155\end{array}$ 



\section{PROBING STRUCTURE IN THE BRAIN WITH DIFFUSION MRI}

\section{I.I STRUCTURE AND FUNCTION IN THE HUMAN BRAIN}

The human brain is a highly complex organ. If we look a little closer inside the brain, we observe that it is not an amorphous mass, but it is organized in gray and white matter and sub-cortical structures. The gray matter, or the cortex, contains the neurons needed for brain function, while the white matter consists of axons organized in fasciculi or fibers and connects several parts of the brain and spinal cord. The sub cortical structures such as the thalamus in figure 1.1 act as relay stations between the cortex and brain stem or between different brain areas.

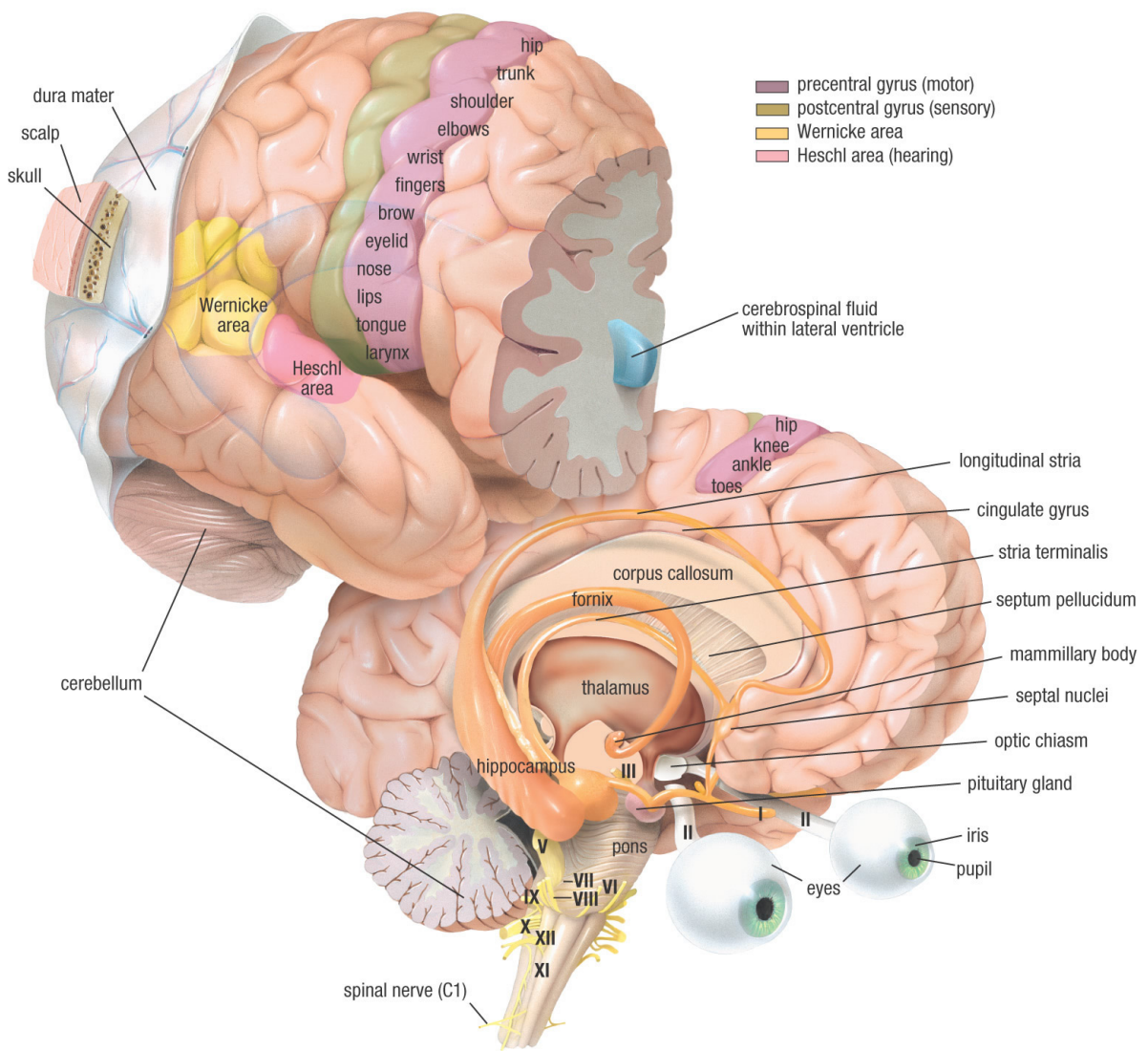

Figure 1.1: A gross overview of the human brain. Image from http://svhrad.com. 


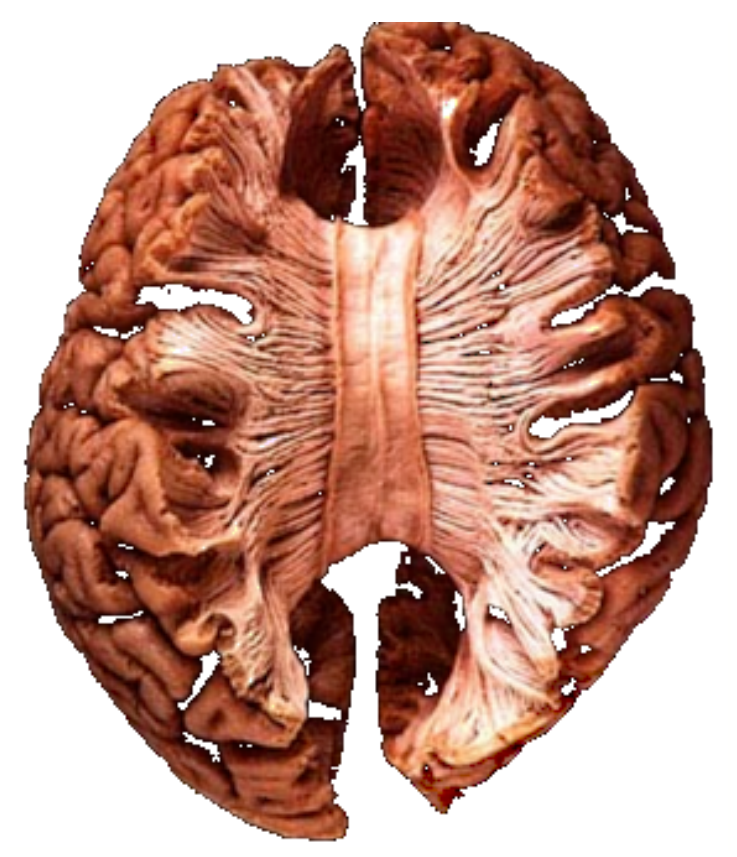

Figure 1.2: Dissection of the brain, showing various bundles in the white matter. Image from http://www.dmi. usherb.ca/ descotea/.

The neurons in the cortex are connected with the rest of the brain and the spinal cord via the white matter. White matter in turn consists axons, some heavily myelinated, organized in fasciculi or fibers. Macroscopically the white matter looks as a homogeneous mass. If we zoom in, we will find that the white matter is organized in bundles, connecting various brain regions, see figure 1.2. Using ex-vivo dissections this bundle organization was made visible already as early as 1672 by Willis [1]. These white matter bundles, some of which are even visible to the naked eye, are in turn made up of tightly packed and highly organized myelinated axons, see figure 1.3.

With magnetic resonance imaging (MRI) it is possible to image structure and function in the brain. In this thesis, we will focus mainly on diffusion-weighted MRI (DW-MRI) to probe for white matter structure of the brain. Because fMRI is used in chapter 5 to probe brain function a short discussion is included next.

\subsection{IMAGING BRAIN FUNCTION WITH FMRI}

BOLD imaging is a well-established, widely used technique to image brain function. The basic principle is that because of neuronal activity the delivery of oxygenated blood to the active region increases, thereby decreasing the amount of de-oxygenated haemoglobin $(\mathrm{Hb})$. Oxygenated blood contains oxy-haemoglobin, which is a diamagnetic substance and has a small effect on the regional magnetic field. Deoxy-haemoglobin on the other hand, is a paramagnetic substance and 

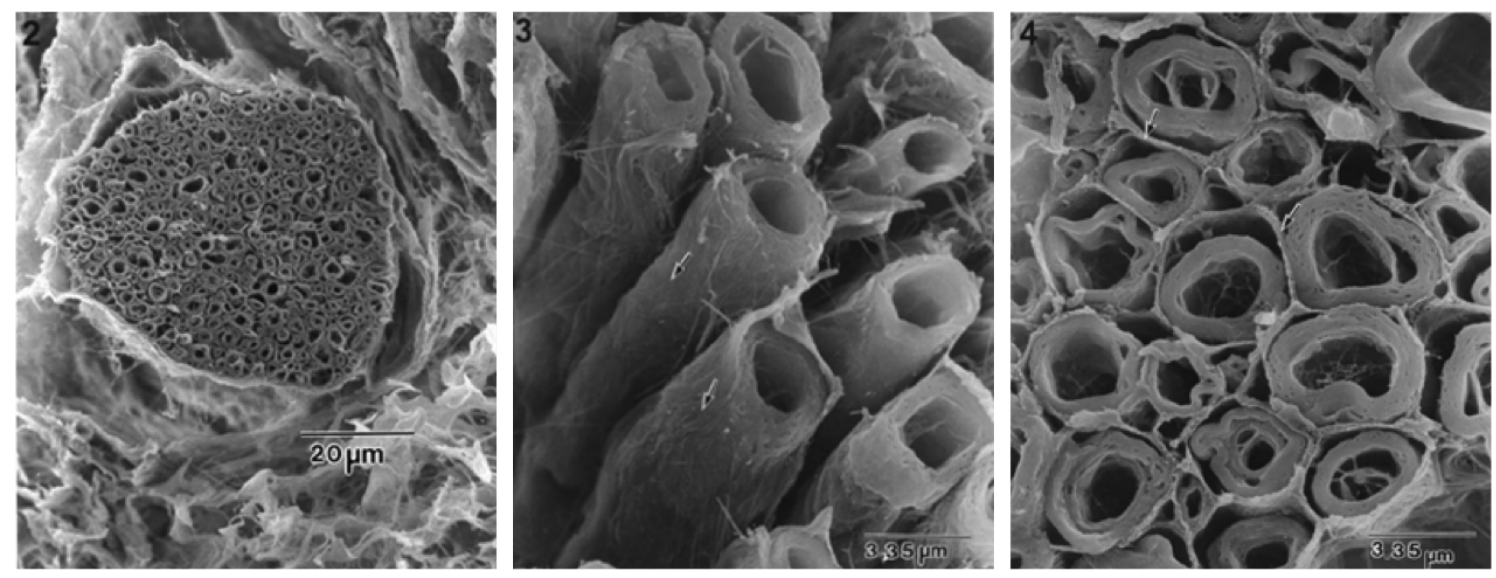

Figure 1.3: Axonal organization in the white matter. The left image shows a tightly packed bundle of axons. Zooming in we can appreciate the tubular structure of the axons.

disturbs the local magnetic field, leading to a significant $T_{2}^{*}$ decrease. The balance of oxy- and de-oxygenated haemoglobin in a voxel determines the voxel's $T_{2}^{*}$ induced image intensity. In summary, local neuronal activation leads to an increase in oxygenated $\mathrm{Hb}$ and a decrease of de-oxygenated $\mathrm{Hb}$, leading to a net $1-10 \%$ image intensity increase.

During an fMRI experiment stimuli are presented to the subject, for instance visually via a screen in the MRI scanner or auditory stimuli via headphones. The exact timing of the stimuli is known to the experimenter and is used later on to perform a statistical analysis on the acquired data. Each echo time (TE, typically 1.5-3 seconds) a whole brain EPI scan is made. The intensity changes in a voxel over time thus form a time course which can be used for the analysis.

The internal white matter structure, connecting different brain regions, can be studied in-vivo since about 15 years with diffusion-weighted MRI, which is discussed in the next section.

\subsection{IMAGING BRAIN STRUCTURE WITH DIFFUSION-WEIGHTED MRI}

The basis of DW-MRI [2] is diffusion of water molecules in the brain. Although not visible to the observer, water molecules in a glass of water move randomly and collide frequently with each other. This phenomenon, self-diffusion of water, was first observed by Brown (1828) and was thereafter termed Brownian motion. Albert Einstein quantified the macroscopic behaviour of water self-diffusion:

$$
\left\langle x^{2}\right\rangle=2 D t
$$

where $\left\langle x^{2}\right\rangle$ is the mean squared displacement along a single dimension $x$ of the water molecules during the diffusion time $t$ and $D$ is the diffusion coefficient. In 


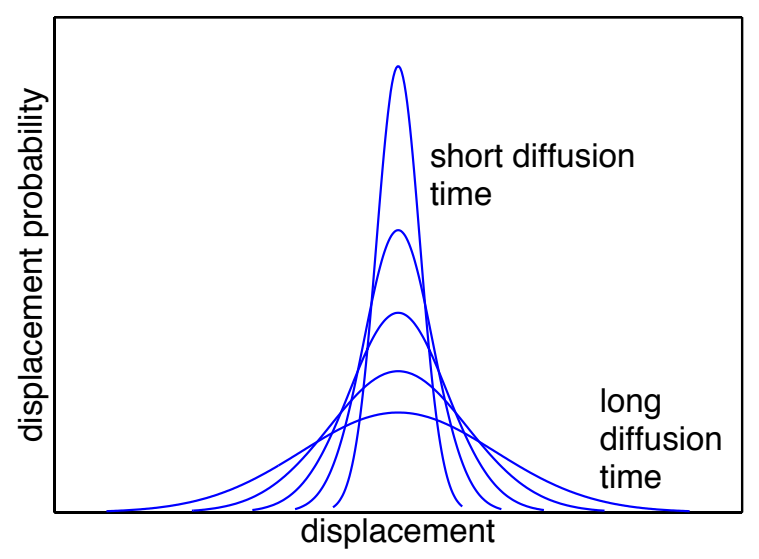

Figure 1.4: The Gaussian diffusion profile in one dimension. For longer diffusion times, the displacement will be larger and the probability of finding a molecule at a location near the starting point of the diffusion path (center of the graph) will be smaller.

an isotropic environment, where the environment is the same in each direction (such as in the center of a glass of water) the diffusion will have a Gaussian profile shown in figure 1.4 in the 1 -dimensional case. If the diffusion time is short, molecules have not moved a long distance on average, but when we wait longer the displacement for many molecules will be larger as well, which implies that the variance in position increases. Assuming a Gaussian diffusion profile, we can describe the probability density function (PDF), or diffusion propagator $\mathcal{P}$ of a water molecule being present at a certain location $x$ at time $t$ :

$$
\mathcal{P}(x, t)=\frac{1}{\sqrt{(4 \pi D t)}} \mathrm{e}^{-x^{2} / 4 D t}
$$

\subsubsection{Diffusion and axons}

The water molecules inside and surrounding the axons are responsible for the signal in DW-MRI. Because of the tubular structure of the axons -an axon is basically a long myelinated tube with an inner diameter in the range of 1 to $20 \mu \mathrm{m}-$, water molecules have a higher probability to diffuse along the long axis of the axon, while the probability of diffusing along the perpendicular axes is lower, see figure 1.5. Many factors such as local structure, axonal membrane integrity, fiber density, degree of myelinisation etc. may contribute to local anisotropic diffusion in the brain. Figure 1.6 shows examples of various diffusion profiles to be found in the brain. The exact mechanism underlying water diffusion in and around axons is not yet clearly understood, but it is shown that the largest factor contributing to anisotropic diffusion is the cell membrane [3]. 


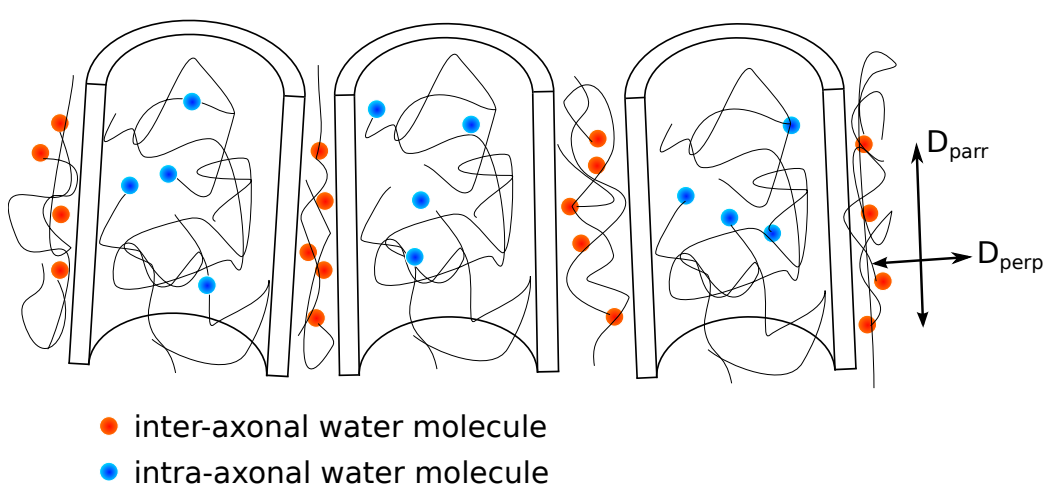

Figure 1.5: Schematic view of inter- and intra-axonal diffusion. Simplified, the axon is a hollow tube covered by myelin sheaths. Water molecules are trapped inside the tube, or in between the axons. A very small amount of water molecules can diffuse through the cell membrane. It is estimated that approximately $20 \%$ of the diffusion signal is caused by inter-axonal diffusion, while $80 \%$ is caused by intra-axonal diffusion [4]. The diffusion constant parallel to the axons is larger than the diffusion constant perpendicular to the axons, because the water molecules can travel further parallel than perpendicular to the axons in a given time period.

\subsubsection{Diffusion and MRI}

The MR signal can be sensitized for diffusion by applying a pair of gradients; the first gradient to de-phase the spins, the second one to re-phase them. If spins do not move during the time between the two gradients, the gradients' effect on the signal is re-phased and $T_{2}$ decay mainly affects the signal. However, when spins do move (diffuse) during this short time, the gradients' effect on the signal is not perfectly re-phased and additional signal is lost, hence we have diffusion weighting in the MR signal.

The Pulsed Gradient Spin Echo (PGSE) or Stejskal-Tanner experiment [6] is the basic pulse sequence in diffusion MR and it is described in figure 1.7. The diffusion gradients can be played out in different directions to sample the diffusion along different directions in space. The diffusion gradient vector is therefore written as a product of the gradient strength and its normalized direction:

$$
\mathbf{g}=G \cdot \hat{\mathbf{g}}
$$

The PGSE signal $E$ is a superposition of the transverse magnetizations with phase $\varphi$

$$
E=E_{0}\left\langle\mathrm{e}^{i \varphi}\right\rangle,
$$




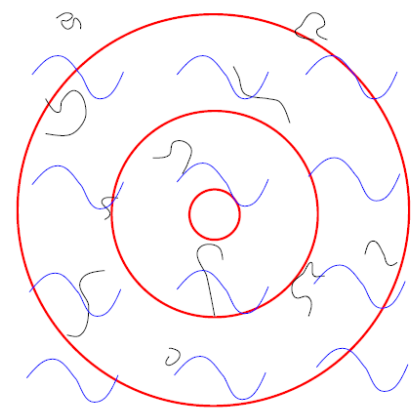

(a)

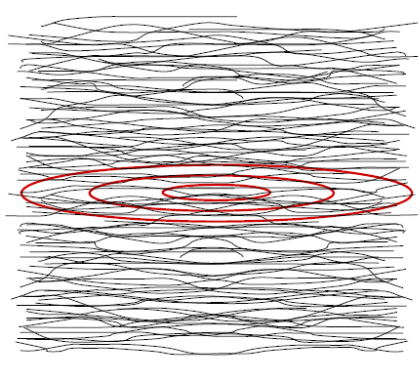

(c)

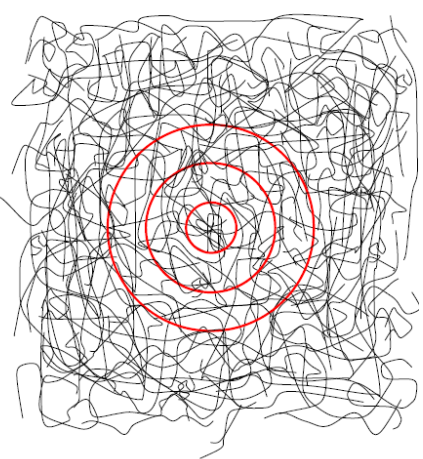

(b)

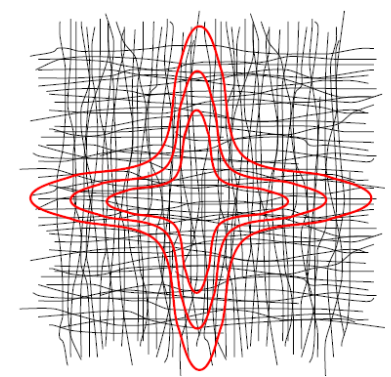

(d)

Figure 1.6: Schematic diagrams of microstructures and diffusion profiles found in the brain. a) shows a fluid filled region (e.g. a ventricle) with very little structure causing isotropic water diffusion; b) shows gray matter, with random oriented structures, causing an macroscopic isotropic diffusion profile c) and d) show highly structured white matter with one or two fiber orientations. Figure reproduced with permission from [5].

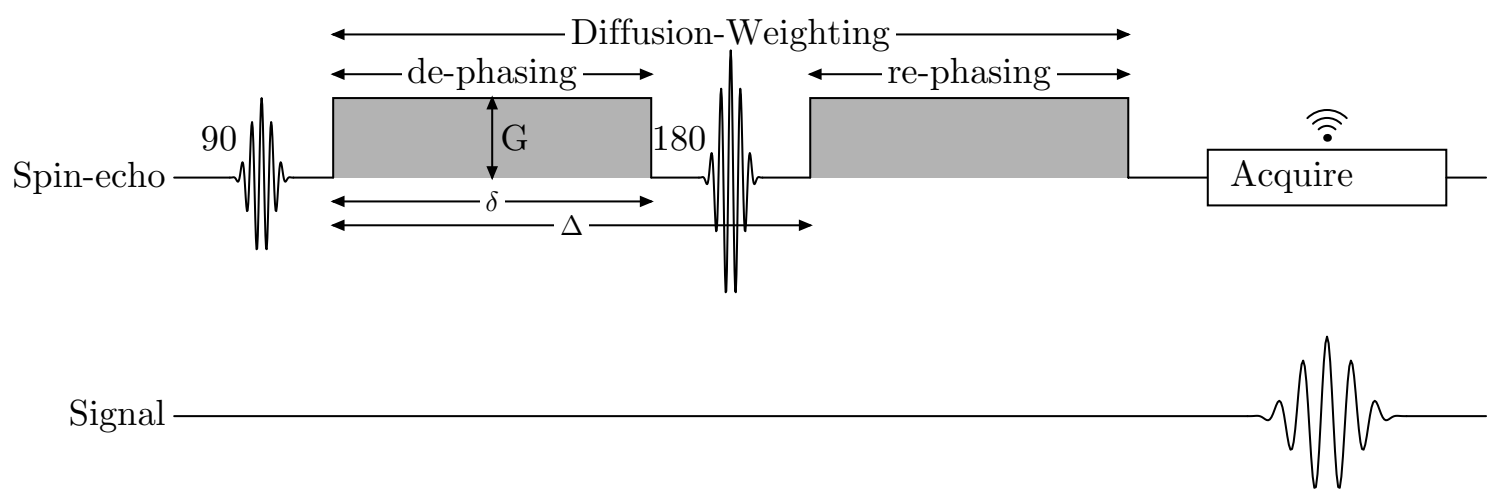

Figure 1.7: The PGSE sequence with two diffusion gradients. $\delta$ : gradient duration [s], G: gradient amplitude $[\mathrm{mT} / \mathrm{m}], \Delta$ effective diffusion time $[\mathrm{s}]$. 
where $E_{0}$ is the signal without diffusion weighting. The spin phase $\varphi$ accumulated during each pulse in a PGSE experiment can described by

$$
\varphi=\gamma \int_{0}^{\delta} \mathbf{g}^{T} \mathbf{x} \mathrm{d} t=\gamma \delta \mathbf{g}^{T} \mathbf{x}
$$

with $\mathbf{g}$ the diffusion gradient vector with strength $G, \mathbf{x}$ the three-dimensional spin position, and $\gamma$ the gyromagnetic ratio $(42.58 \mathrm{MHz} / \mathrm{T})$. During the entire experiment, the total accumulated phase is then

$$
\begin{aligned}
\varphi & =\gamma \delta \mathbf{g}^{T}(\mathbf{x}(0)-\mathbf{x}(t=\Delta)) \\
\text { substituting } \mathbf{r} & =\mathbf{x}(0)-\mathbf{x}(t=\Delta) \\
\varphi & =\gamma \delta \mathbf{g}^{T} \mathbf{r},
\end{aligned}
$$

The spin position is assumed to be constant in the time period $\delta$ when the gradient is turned on, so $\mathbf{x}(0)$ is the spin position during the first gradient and $\mathbf{x}(t=\Delta)$ the spin position during the second gradient pulse. From this it immediately follows that a change in phase can only occur if spins move during the time between the two gradients.

The signal in a DW-MRI experiment is then the product of the signal phase, given above, and the population of spins, given by equation. 1.2, but now in three dimensions and integrated over all positions [7]:

$$
S=\int \mathcal{P}(\mathbf{r}, t) \varphi(\mathbf{r}) \mathrm{d} \mathbf{r}=\frac{1}{\sqrt{4 \pi D \Delta}} \int \mathrm{e}^{-\mathbf{r}^{2} / 4 D \Delta} \mathrm{e}^{i \gamma \mathbf{g} \delta \mathbf{r}} \mathrm{d} \mathbf{r}
$$

From the above can be observed that the largest change in phase is obtained when spins move in the same direction as the gradient direction. Contrast is determined by the product of spin displacement and the product of the gradient strength $G$ and its duration $\delta$. Therefore, it is desirable to have gradients switched on as long as possible (increase $\delta$ ) or to increase the gradient strength.

In the brain spin motions are obviously not aligned along the gradient direction, so if we want to measure the diffusion of spins in the brain, we have to sample along multiple gradient directions. In order to reconstruct the diffusion profile in a particular voxel, the direction of the gradient $\mathbf{g}$ is changed. For diffusion tensor imaging (DTI) [8] at least 6 unique gradient directions are required, but to improve the robustness of tensor estimation, it is advised to use at least 30 unique gradient directions [9]. Sampling with more than 30 unique gradient directions does not significantly improve data quality for DTI applications, but for more advanced reconstruction algorithms, this is beneficial. Sampling with a lot of gradient directions is commonly called High Angular Resolution Diffusion Imaging (HARDI). 


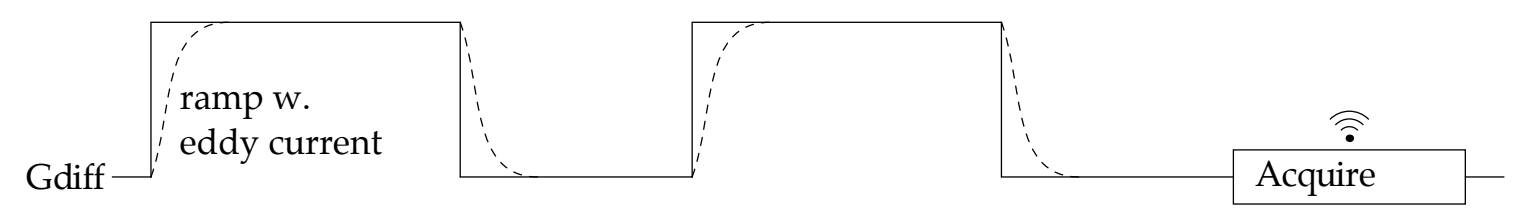

Figure 1.8: Gradient waveform due to eddy currents (figure adapted from [10].

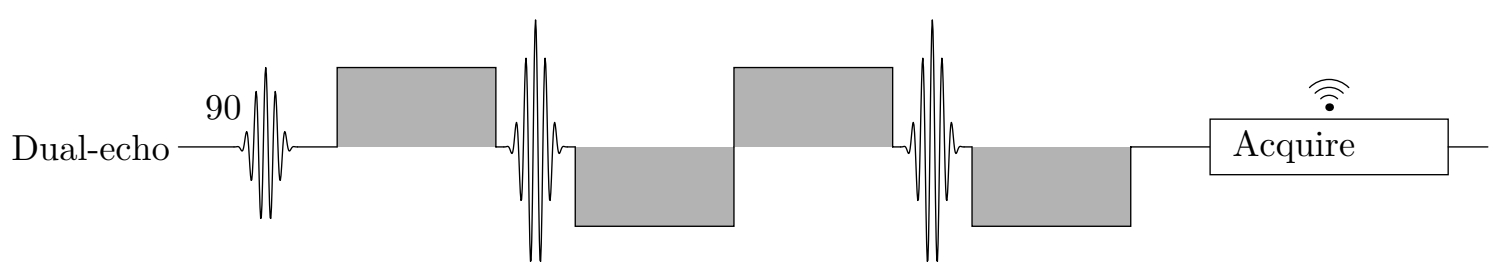

Figure 1.9: Dual echo or Double-refocussed diffusion weighted spin-echo sequence to reduce the effects of eddy currents by introducing bipolar gradient pairs.

\section{Practical Aspects of DW-MRI Acquisition}

The rapid switching of the large gradients used in DW-MRI induces currents in conducting elements (cryostat, RF coils, cooling elements etc) in the MR scanner [10] called eddy currents. More exactly, it is the gradient slew (change) that causes these currents. Deformation of the gradient ramp is shown in figure 1.8 as a deviation from the boxcar shape which is ideally desired. Eddy currents are roughly proportional to the slew rate and slew duration, which is equal to the amount of change in gradient amplitude [3]. The result of eddy currents is that the actual gradient played out by the scanner looks different from the one that was programmed in the sequence. Figure 1.8 shows an exaggerated view of the gradient shape (dashed line) as a result of eddy currents.

The result of long time constant eddy currents that overlap with the EPI readout train is a shape deformation -scaling, translation or shear- of the image and can be corrected for in the post-processing of the data, see chapter 4 of this thesis. Another solution is to improve the DW-MRI acquisition protocol, and a widely used solution is to use positive and negative (i.e. bipolar) gradient pairs, see figure 1.9, which tend to cancel out each other's eddy currents [3].

GRADIENT NON-LINEARITIES A second source of errors in DW-MRI arises from gradient non-linearities, that is the fidelity of the spatial gradient shape. A gradient coil is built to have a linearly changing magnetic field within the fieldof-view (FOV) which is required. Outside the FOV, the field should reduce as fast as possible [3]. Inside the FOV, the gradient is not perfectly linear, which has consequences for the $b$-value, which is related to the square of the gradient strength $G^{2}$, see equation 1.14. The three gradients $G_{x}, G_{y}, G_{z}$ present in the MR 

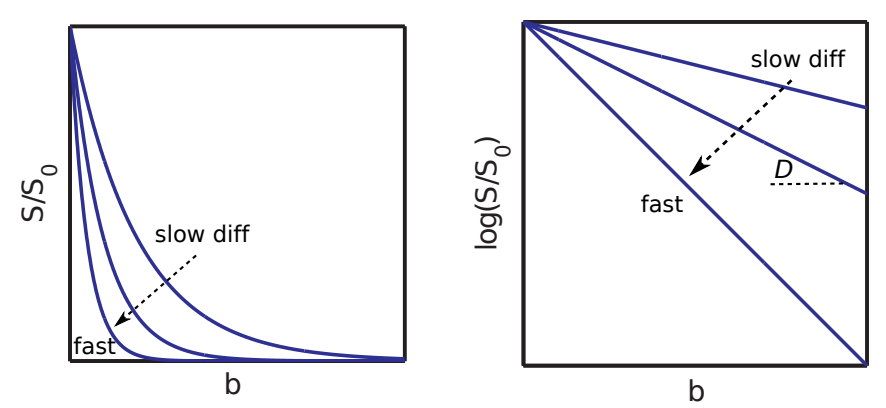

Figure 1.10: The signal as function of the $b$-value. The signal decreases exponentially as the $b$-value is increased. The diffusion constant $D$ is the slope of the logarithm of the signal.

system are likely to differ slightly from each other, causing slight differences in the $b$ - value and direction of the diffusion gradient.

\section{Diffusion MRI Reconstruction Algorithms}

THE DIFFUSION TENSOR If we assume a Gaussian diffusion profile, we can combine Eqs. 1.2 and 1.9 to obtain the diffusion coefficient $D$. Note that the diffusion coefficient is not the 'real' diffusion coefficient, but a calculated, apparent diffusion coefficient $(A D C)$.

$$
\begin{aligned}
\frac{S}{S_{0}} & =\mathrm{e}^{-\gamma^{2} G^{2} \delta^{2}(\Delta-\delta / 3) D} \\
\text { substituting } b & =\gamma^{2} G^{2} \delta^{2}(\Delta-\delta / 3) \\
S & =S_{0} \mathrm{e}^{-b D} \\
\ln \left(\frac{S}{S_{0}}\right) & =-b D \\
\ln (S) & =\ln \left(S_{0}\right)-b D
\end{aligned}
$$

where we have come across the all-important $b$-factor

$$
b=\gamma^{2} G^{2} \delta^{2}(\Delta-\delta / 3)
$$

By changing the $b$-value -that is, by changing $\delta, \Delta$ or $G$ - we can change the amount of diffusion weighting, and the signal we will measure will be different. As illustrated in figure 1.10, a higher b-value will result in a faster signal decay. When $b=0$, we do not apply any diffusion weighting. The choice of $b$ has important consequences for post-processing of the data as we will see in a later section. 
The symmetric three-by-three diffusion tensor $\mathbf{D}$ describes the Gaussian diffusion profile

$$
\mathbf{D}=\left[\begin{array}{lll}
D_{x x} & D_{x y} & D_{x z} \\
D_{y x} & D_{y y} & D_{y z} \\
D_{z x} & D_{z y} & D_{z z}
\end{array}\right] .
$$

To fill $\mathbf{D}$ we need at least six measurements, plus a measurement without diffusion weighting. In practice usually more than six measurements are acquired, and the tensor $\mathbf{D}$ is obtained in the simplest case from linear least-squares estimation of Eq. 1.13. If $\mathbf{X}$ is a vector containing the log-transformed signal intensities for $N$ gradient directions, that is $\mathbf{X}_{i}=\ln \left(S_{i} / S_{0}\right), i=1 . . N$, the $b$-matrix a combination of the gradient directions and the $b$-value [3],

$$
\begin{aligned}
\mathbf{B}= & b \cdot\left[\begin{array}{cccccc}
g_{x}^{1} g_{x}^{1} & 2 g_{x}^{1} g_{y}^{1} & 2 g_{x}^{1} g_{z}^{1} & g_{y}^{1} g_{y}^{1} & 2 g_{y}^{1} g_{y}^{1} & g_{z}^{1} g_{z}^{1} \\
g_{x}^{2} g_{x}^{2} & 2 g_{x}^{2} g_{y}^{2} & 2 g_{x}^{2} g_{z}^{2} & g_{y}^{2} g_{y}^{2} & 2 g_{y}^{2} g_{y}^{2} & g_{z}^{2} g_{z}^{2} \\
\vdots & \vdots & \vdots & \vdots & \vdots & \vdots \\
g_{x}^{N} g_{x}^{N} & 2 g_{x}^{N} g_{y}^{N} & 2 g_{x}^{N} g_{z}^{N} & g_{y}^{N} g_{y}^{N} & 2 g_{y}^{N} g_{y}^{N} & g_{z}^{N} g_{z}^{N}
\end{array}\right] \\
= & {\left[\begin{array}{cccccc}
b_{x x}^{1} & b_{x y}^{1} & b_{x z}^{1} & b_{y y}^{1} & b_{y z}^{1} & b_{z z}^{1} \\
b_{x x}^{2} & b_{x y}^{2} & b_{x z}^{2} & b_{y y}^{2} & b_{y z}^{2} & b_{z z}^{2} \\
\vdots & \vdots & \vdots & \vdots & \vdots & \vdots \\
b_{x x}^{N} & b_{x y}^{N} & b_{x z}^{N} & b_{y y}^{N} & b_{y z}^{N} & b_{z z}^{N}
\end{array}\right] }
\end{aligned}
$$

then we can solve for $\mathbf{D}$ according to the expression

$$
\begin{aligned}
& \mathbf{X}=\mathbf{B D} \\
& \mathbf{D}=\mathbf{B}^{-1} \mathbf{X} \text { for } N=6 \\
& \mathbf{D}=\left(\mathbf{B}^{T} \mathbf{B}\right)^{-1} \mathbf{B}^{T} \mathbf{X} \text { for } N>6 .
\end{aligned}
$$

The eigenvalues $\lambda_{1} \geq \lambda_{2} \geq \lambda_{3}$ of $\mathbf{D}$ represent the shape of the tensor. The corresponding eigenvectors $\mathbf{v}_{1}, \mathbf{v}_{2}, \mathbf{v}_{3}$ represent the orientation of the tensor in three-dimensional space.

To highlight specific features of the tensor scalar measures were introduced. The trace of the diffusion tensor

$$
\operatorname{Tr}(\mathbf{D})=D_{x x}+D_{y y}+D_{z z}
$$

indicates the absolute amount of diffusion in that voxel. The related measure Mean Diffusivity is defined as

$$
M D=\langle\operatorname{Tr}(\mathbf{D})\rangle=\operatorname{Tr}(\mathbf{D}) / 3 .
$$



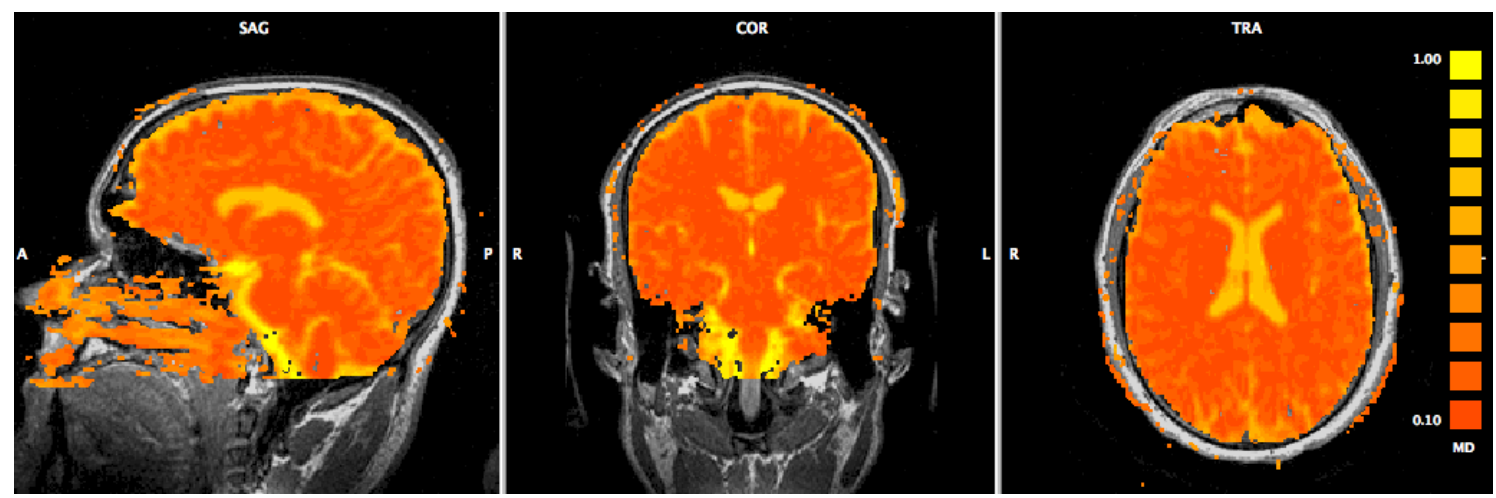

Figure 1.11: MD map. Diffusivity is higher in regions where diffusion is free, e.g. the ventricles and lower in regions where it is more restricted, e.g. in white matter.
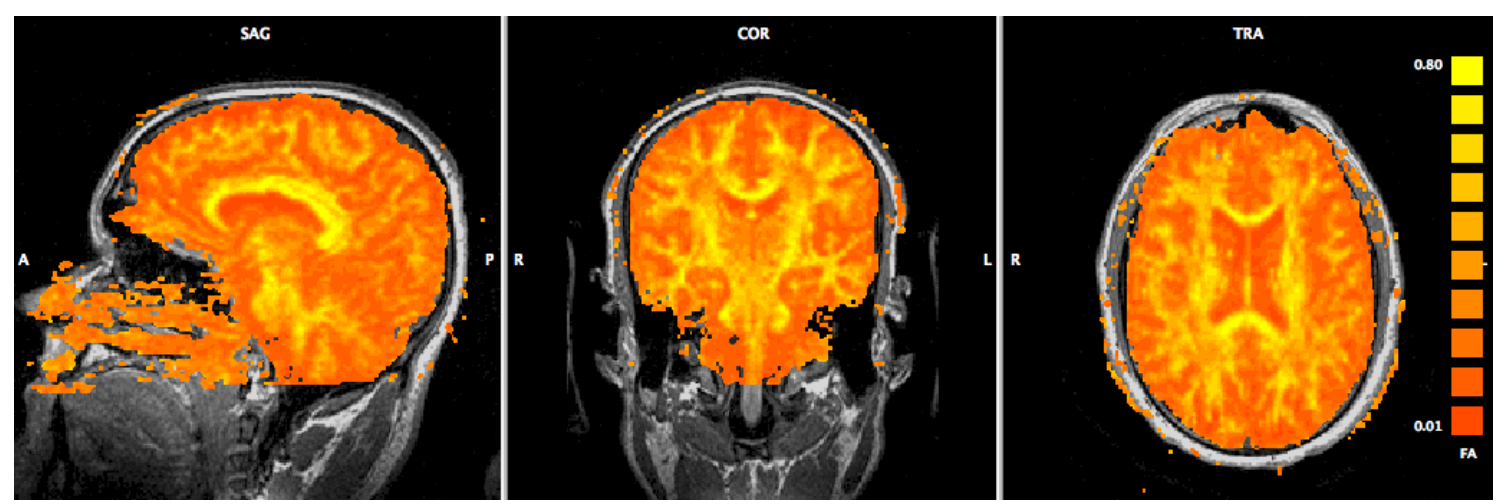

Figure 1.12: FA map. Higher FA values indicate more anisotropic diffusion in a voxel.

Fractional anisotropy $(F A)$ is the normalized standard deviation of the eigenvalues, and defined as

$$
F A=\sqrt{\frac{1}{2}} \frac{\sqrt{\left(\left(\lambda_{1}-\lambda_{2}\right)^{2}+\left(\lambda_{2}-\lambda_{3}\right)^{2}+\left(\lambda_{3}-\lambda_{1}\right)^{2}\right)}}{\sqrt{\lambda_{1}^{2}+\lambda_{2}^{2}+\lambda_{3}^{2}}}
$$

If we color code the FA map by mapping the direction of the principal eigenvector to an RGB color map, we obtain the familiar FA image in figure 1.13. If the largest eigenvector points in the left-right $(x)$ direction, color is red; if it points in the anterior-posterior $(y)$ direction, color is green; if it points in the inferiorsuperior $(z)$ direction it is blue. Finally, the shape of the tensor can be described 

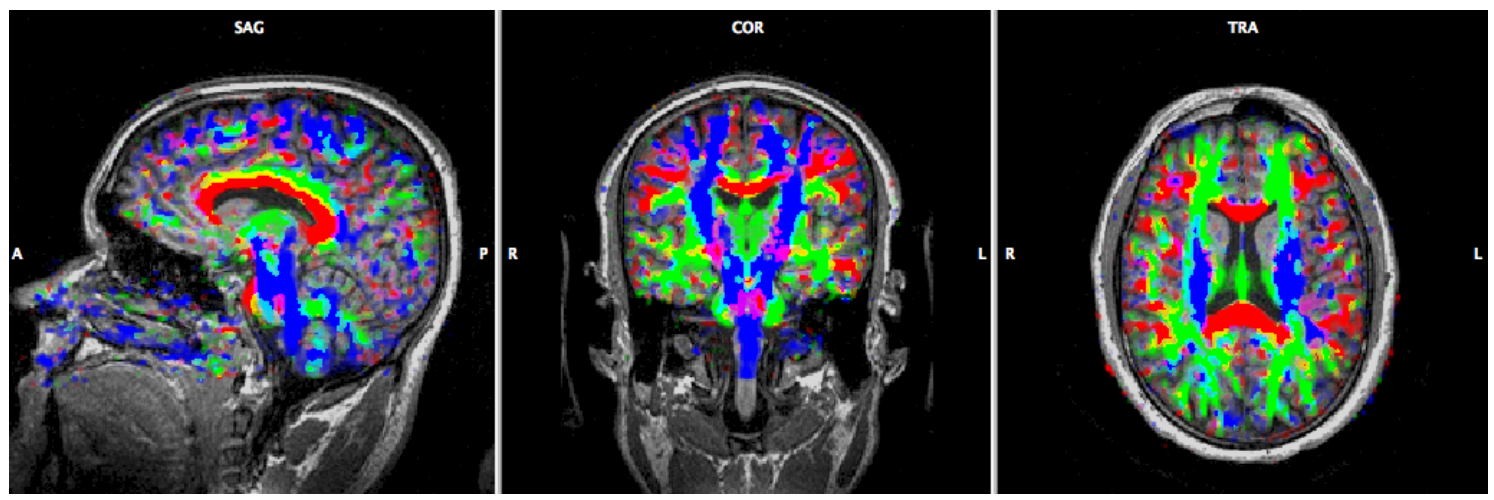

Figure 1.13: RGB color coded FA map. To emphasize structure, the colors in the map are weighted by the FA value.
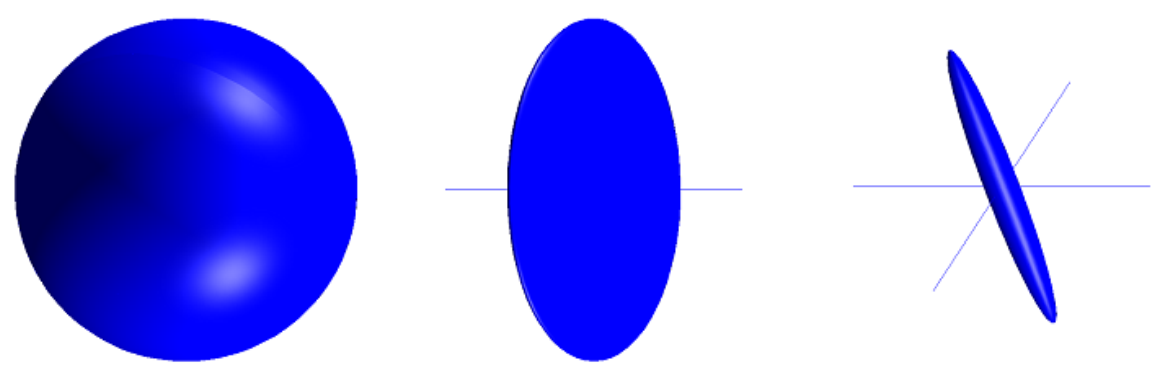

Figure 1.14: Spherical (high $c_{s}$ ), planar (high $c_{p}$ ) and linear (high $c_{l}$ ) tensor shapes.

by shape indices, introduced by Westin et al. [11]:

$$
\begin{aligned}
\text { linear: } c_{l} & =\frac{\lambda_{1}-\lambda_{2}}{\lambda_{1}} \\
\text { planar: } c_{p} & =\frac{\lambda_{2}-\lambda_{3}}{\lambda_{1}} \\
\text { spherical: } c_{s} & =\frac{\lambda_{3}}{\lambda_{1}} .
\end{aligned}
$$

Q-BALL IMAGING The Orientation Density Function (ODF) is a radial projection of the PDF $\mathcal{P}$ onto the unit sphere. It expresses the probability of a spin displacing in a certain unit normalized fiber direction $\hat{\mathbf{x}}$ [12]. The ODF has maxima in the directions where $\mathcal{P}$ has most mass and therefore it is assumed that these are the fiber directions [13].

$$
\psi(\hat{\mathbf{x}})=\int_{0}^{\infty} \mathcal{P}(\alpha \hat{\mathbf{x}}) \mathrm{d} \alpha,
$$


In Diffusion Spectrum Imaging (DSI) [14] the MR signal is acquired on a large grid of points in $q$-space. $q$-space spans the whole space where a measurement can be made. Every point is defined by a wave vector $\mathbf{q}=\gamma \delta \mathbf{g}$, which can be expressed as a wave number $|\mathbf{q}|$ and a diffusion encoding direction $\hat{\mathbf{q}}=$ $\mathbf{q} /|\mathbf{q}|$. In each point a value of $\mathcal{P}$ is obtained from the signal via a fast Fourier transform. The ODF $\psi$ is computed directly from an interpolation of the PDF $\mathcal{P}$. The disadvantage of DSI is that it requires a large number of measurements and therefore has an acquisition time which is unsuitable for clinical and neuroscientific applications.

Q-ball imaging solves the problem of a large number of measurements by approximating the ODF by a Funk transform (FRT) at a fixed sphere in $q$-space. The value of the Funk transform at a point $\hat{\mathbf{x}}$ is the integral over the great circle perpendicular to $\hat{\mathbf{x}}[13]$. The ODF $\psi$ and the FRT $\mathcal{G}$ are related via

$$
\psi(\hat{\mathbf{x}})=\mathcal{G}_{q^{\prime}}[E(\mathbf{q})]=2 \pi q^{\prime} \int \mathcal{P}(r, \theta, z) J_{0}\left(2 \pi q^{\prime} r\right) r \mathrm{~d} r \mathrm{~d} \theta \mathrm{d} z,
$$

where $J_{0}$ is the zeroth-order Bessel function [15].

DIFFUSION ORIENTATION TRANSFORM (DOT) In DOT, the ODF is not a radial projection of the diffusion propagator, but corresponds to the amplitude of the propagator at a particular displacement $R_{0}$ [16]. The Fourier transform of the water displacement probability as introduced in equation 1.9, can be re-written as

$$
\mathcal{P}(\mathbf{r})=\int E(\mathbf{q}) \mathrm{e}^{-2 i \pi \mathbf{q r}} \mathrm{d} \mathbf{q} .
$$

The DOT transform is then written as a Laplace series expansion of $\mathcal{P}\left(R_{0} \mathbf{r}\right)$ (for derivation see [17]):

$$
\mathcal{P}\left(R_{0} \mathbf{r}\right)=\sum_{l=0}^{\infty} \sum_{m=-l}^{l}(-1)^{1 / 2} \alpha_{l l m}
$$

where the coefficients $\alpha_{l l m}$ are given by a spherical harmonics transform [17].

\section{DTI Based Fiber Tracking}

In DTI we assume that the diffusion tensor represents the underlying fiber structure. Therefore the direction of the diffusion tensor (the direction of the largest eigenvector) represents the direction of a macroscopic neuronal structure. Fiber tracking or tractography is the technique to reconstruct these virtual 3 -D fiber tracts by connecting the eigenvectors in some way.

STREAMLINE TRACTOGRAPHY: THEORY The most widely used and simplest form of fiber tracking simply connects the first eigenvectors. The eigenvectors in 
each voxel in the imaging volume can be regarded as a vector field. The fiber orientation in a voxel is assumed to be the tangent of the streamline, so we can write the streamline $\mathbf{r}(s)$, where $s$ is the location along the streamline, as a differential equation

$$
\frac{\mathrm{d} \mathbf{r}(s)}{\mathrm{d} s}=F(\mathbf{D}) \cdot \mathbf{r}(s),
$$

where $F(\mathbf{D})$ is a function that maps the eigenvalues.

To obtain a smooth line, the vector field is interpolated (i.e. by Euler integration) and a streamline is started from a seed point $\mathbf{x}_{0}=\left(x_{0}, y_{0}, z_{0}\right)$. A tract $\left\{\mathbf{x}_{0}, \mathbf{x}_{1}, \ldots\right\}$ is obtained iteratively (in two directions from the seed point, because the tensor does not have a polarity) as follows [11]:

$$
\begin{aligned}
\mathbf{x}_{k+1} & =\mathbf{x}_{k}+\alpha \mathbf{u}_{k} \\
\mathbf{D}_{k+1} & =F\left(\mathbf{D}\left(\mathbf{x}_{k+1}\right)\right) \\
\mathbf{u}_{k+1} & =\mathbf{D}_{k+1} \mathbf{u}_{k}
\end{aligned}
$$

where $\mathbf{D}\left(\mathbf{x}_{k+1}\right)$ is the diffusion tensor at spatial position $\mathbf{x}_{k+1}$ and $\mathbf{u}_{k+1}$ the direction of the tract. We can for instance follow the major eigenvector in each voxel by setting

$$
F(\mathbf{D})=\lambda_{1} \mathbf{v}_{1} \mathbf{v}_{1}^{T},
$$

this projects any vector onto the direction of the major eigenvector. However, we run into trouble when the tensor is planar instead of prolate, that is when $\lambda_{1}=\lambda_{2}$. If a smarter projection operator is chosen [11],

$$
F(\mathbf{D})= \begin{cases}c_{l} \mathbf{D}_{l} & \text { if } c_{l}>c_{p}, c_{s} \\ c_{p} \mathbf{D}_{p} & \text { if } c_{p}>c_{l}, c_{s} \\ c_{s} \mathbf{D}_{s} & \text { if } c_{s}>c_{l}, c_{p}\end{cases}
$$

the tensor is forced into one of three possible shapes of the eigenvector, which enables the tract to proceed through regions where the tensor is planar or spherical.

STREAMLINE FIBER TRACKING: PRACTICE As we have seen in the previous section, a streamline fiber tract is started from a seed point and propagates bidirectionally in the imaged volume. Locating the right tract can be achieved in two ways: either by calculating all tracts in advance, by setting one or more seed points in every voxel, or by calculating the tracts from regions-of-interest (ROIs) containing seed points. In both cases, the positioning of the ROI is vital for a satisfying result. At best, the ROI encompasses a single fasciculus in the brain, but this is a very difficult task, even for a skilled tractographer. In most cases, multiple ROIs are used and boolean operators AND, NOT are applied to the tracts 


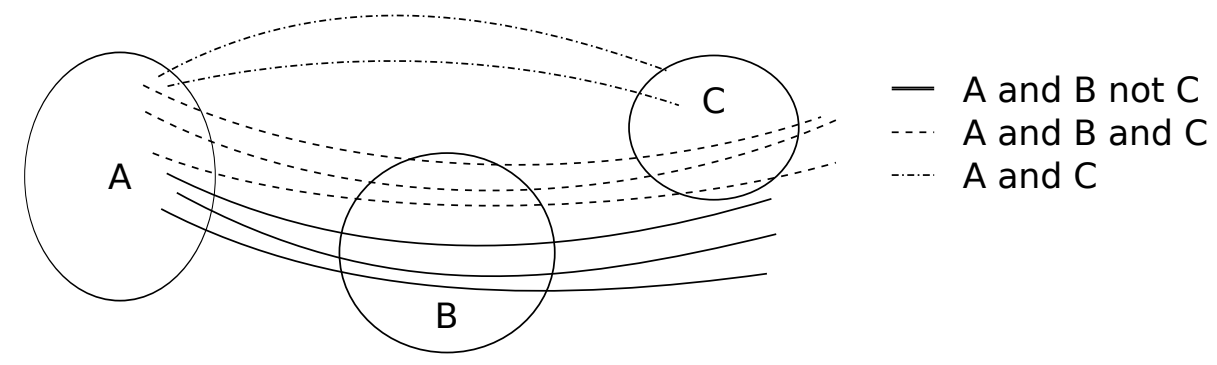

Figure 1.15: Illustration of AND and NOT operations. The fibers originating from area A form a bundle in the direction of areas B and C. Boolean operations are used to pick out the three different fiber bundles.

to separate them, which is illustrated in figure 1.15. An AND operation is used to find tracts between two areas, and a NOT operation is used to exclude tracts passing through a certain area. Since DTI-based fiber tracking has no directionality, it is common practice to combine (A AND B) with (B AND A) operations.

To constrain the tractography stopping criteria are applied. The stopping threshold prevents the tracts from entering the grey matter and is usually defined as an FA threshold $(F A>0.15-0.20)$. The angular threshold prevents the tract from making "infeasible" angles, i.e. by making a short distance 180 degree turn. There is, however, no evidence in the literature at this moment that this is a realistic criterion.

PROBABILISTIC FIBER TRACKING Instead of starting a single fiber tract from a seed point, probabilistic methods sample a large number of possible tracts and then characterize the average properties of that set. The general idea is to, at each iteration of the algorithm, draw the next direction of the tract from a distribution of possible directions [18]. A widely used method [19] assumes that the uncertainty in fiber orientation can be estimated by a simple partial volume model and a noise model. The fiber orientation distribution is then estimated using a Bayesian approach and Monte Carlo sampling [19].

The result of probabilistic tracking is a visitation map. That is, the map simply represents the number of times a voxel is visited by a path in the sampled set and it characterizes both the most visited path between the seed voxel and other voxels in the volume and the uncertainty of variation in that path.

\section{Validation of DW-MRI acquisition and processing}

As we have seen in the previous sections, DW-MRI acquisition is not free from errors. Some of the issues such as the influence of eddy currents can be resolved during the acquisition or by post-processing, wile others such as gradient nonlinearities are currently not corrected for in a clinical setting [3]. Limitations in 
patient safety, scanner hardware, and the choice of $b$-value, number of diffusion directions and usage of parallel imaging complicate the acquisition even further.

The reconstruction of the diffusion profile requires a choice between the available models; to start with, the choice between a Gaussian or a non-Gaussian diffusion model. Even when a well-founded choice is made it remains unclear to what degree the model reflects the true tissue micro-structure.

In case of fiber tracking, the reconstructed fiber tracts may not fully represent the underlying nerve tissue structure, since the amount of signal in a voxel corresponds to the macroscopic, gross summation of all diffusion occurring at a scale several orders of magnitude smaller than the voxel size. This limits the tensor-based fiber tracking technique from resolving complex white matter cytoarchitecture, such as diverging or crossing nerve fibers. Furthermore, the tracts are reconstructed, virtual representations of nerve fiber bundles. The tracts are reconstructed upon constraints set by the user and depend on data quality as well as tracking algorithm. It is, thus, important to assess the quality of fiber tracking results not only qualitatively, but also to be able to quantify precision, reproducibility and accuracy [20]. Because of these issues, validation of DW-MRI acquisition, processing and DW-MRI based fiber tracking is of great importance.

In conclusion we can state that given the various acquisition parameters, diffusion models and tractography methods, there is an important need to verify the before mentioned methods using hardware or software models, comparative post-mortem anatomical studies, and in an ideal world, a combination of the techniques.

\subsection{THESIS OUTLINE}

This thesis is made up of two distinct parts; in the first part, we will discuss validation and correction of DWI acquisition. In Chapter 2, we present an anisotropic hardware phantom for validation of DW-MRI acquisition and processing. An anisotropic diffusion phantom is used in chapter 3 to investigate the effect on DW-MRI measures of orientation of an anisotropic object relative to the main magnetic field. In chapter 4 , we propose a post-processing method to correct for subject motion and distortions due to eddy currents in DW-MRI data.

In the second part of this thesis, we present application studies: in chapter 5 the letter-sound integration in a recovered Landau-Kleffner patient was investigated. The next chapter, chapter 6, focusses on the underlying white matter tracts of the language system of the same patient and were compared to a reference atlas build on a population of healthy subjects. In chapter 7 , fiber tracts in the visual system of a blindsight patient were identified and compared to five control subjects.

Finally, the last chapter provides a general discussion followed by recommendations and future directions. 


\section{REFERENCES}

[1] JD Schmahmann and DN Pandya. Cerebral white matter-historical evolution of facts and notions concerning the organization of the fiber pathways of the brain. Journal of the history of the neurosciences, 16(3):237-67, 2007.

[2] D Le Bihan, E Breton, D Lallemand, P Grenier, E Cabanis, and M LavalJeantet. MR imaging of intravoxel incoherent motions: application to diffusion and perfusion in neurologic disorders. Radiology, 161:401-407, 1986.

[3] H Johansen-Berg and TEJ Behrens. Diffusion MRI. Academic Press, London, 2009.

[4] C Beaulieu. The basis of anisotropic water diffusion in the nervous system-a technical review. NMR Biomed., 15(7-8):435-455, 2002.

[5] DC Alexander. Visualization and image processing of tensor fields, chapter An introduction to computational diffusion MRI: the diffusion tensor and beyond. Springer, 2006.

[6] EO Stejskal and JE Tanner. Spin diffusion measurements: Spin echoes in the presence of a time-dependent field gradient. J Chemical Physics, 42:288-292, 1965.

[7] S Mori. Introduction to Diffusion Tensor Imaging. Elsevier, 2007.

[8] PJ Basser, J Mattiello, and D Lebihan. MR Diffusion Tensor Spectroscopy and Imaging. Biophysical Journal, 66(1):259-267, 1994.

[9] DK Jones. The effect of gradient sampling schemes on measures derived from diffusion tensor MRI: a Monte Carlo study. Magn Reson Med, 51:807815, 2004.

[10] D Le Bihan, C Poupon, A Amadon, and F Lethimonnier. Artifacts and pitfalls in diffusion MRI. Journal of Magnetic Resonance Imaging, 24(3):478$88,2006$.

[11] C Westin, SE Maier, H Mamata, A Nabavi, FA Jolesz, and R Kikinis. Processing and visualization for diffusion tensor MRI. Medical Image Analysis, 6:93-108, 2002.

[12] D Tuch. Diffusion MRI of complex tissue structure. PhD thesis, Harvard-MIT Division of Health Sciences and Technology, 2002.

[13] DC Alexander. Multiple-fiber reconstruction algorithms for diffusion MRI. Annals of the New York Academy of Sciences, 1064:113-33, December 2005. 
[14] VJ Wedeen, P Hagmann, WY Tseng, TG Reese, and RM Weisskoff. Mapping complex tissue architecture with diffusion spectrum magnetic resonance imaging. Magn. Reson. Med., 54:1377-1386, 2005.

[15] D Tuch. Q-ball imaging. Magn. Reson. Med., 52:1358-1372, 2004.

[16] J-D Tournier, S Mori, and A Leemans. Diffusion tensor imaging and beyond. Magn Reson Med, 2011.

[17] E Ozarslan, TM Shepherd, BC Vemuri, SJ Blackband, and TH Mareci. Resolution of complex tissue microarchitecture using the diffusion orientation transform (DOT). Neuroimage, 31:1086-1103, 2006.

[18] DK Jones. Studying connections in the living human brain with diffusion MRI. Cortex, 44:936-952, 2008.

[19] TEJ Behrens, MW Woolrich, M Jenkinson, RG Nunes, S Clare, PM Matthews, JM Brady, and SM Smith. Characterization and Propagation of Uncertainty in Diffusion-Weighted MR Imaging. Magn Reson Med, 1088:1077-1088, 2003.

[20] A Leemans, J Sijbers, M Verhoye, A Van Der Linden, and D Van Dyck. Mathematical framework for simulating diffusion tensor MR neural fiber bundles. Magn Reson Med, 53(4):944-53, 2005. 
Part I

VALIDATION \& CORRECTION OF DIFFUSION MRI ACQUISITION 

GROUND TRUTH HARDWARE PHANTOMS FOR VALIDATION OF DIFFUSION-WEIGHTED MRI APPLICATIONS

This Chapter is based on P Pullens, A Roebroeck and R Goebel. Ground Truth Hardware Phantoms for Validation of Diffusion-Weighted MRI Applications. J Magn Reson Imag, 32:482-488, 2010 


\section{ABSTRACT}

To quantitatively validate diffusion-weighted MR imaging (DW-MRI) applications, a hardware phantom containing crossing fibers at a sub-voxel level is presented. It is suitable for validation of a large spectrum of DW-MRI applications from acquisition to fiber tracking, which is an important recurrent issue in the field.

Phantom properties were optimized to resemble properties of human white matter in terms of anisotropy, Fractional Anisotropy and $T_{2}$. Sub-voxel crossings were constructed at angles of 30, 50 and 65 degrees, by wrapping polyester fibers, with a diameter close to axon diameter, into heat shrink tubes. We show our phantoms are suitable for the acquisition of DW-MRI data using a clinical protocol.

The phantoms can be used to succesfully estimate both the diffusion tensor and non-gaussian diffusion models, and perform streamline fiber tracking. DOT (Diffusion Orientation Transform) and Q-ball reconstruction of the diffusion profiles acquired at $b=3000 \mathrm{~s} / \mathrm{mm}^{2}$ and 132 diffusion directions reveal multimodal diffusion profiles in voxels containing crossing yarn strands.

The highly-purpose adaptable phantoms provide a DW-MRI validation platform: applications include optimisation of acquisition schemes, validation of non-gaussian diffusion models, comparison and validation of fiber tracking algorithms and quality control in multi-center DWI studies.

\subsection{INTRODUCTION}

Diffusion tensor-based fiber tracking is limited in resolving complex white matter cytoarchitecture, such as diverging or crossing nerve fibers. Furthermore, the tracts are virtual representations of nerve fiber bundles, depending on data quality as well as tracking algorithm and its user-set parameters. It is important to assess the quality of fiber tracking results not only qualitatively, but also to be able to quantify precision, reproducibility and accuracy [1].

Hardware diffusion phantoms have the important advantage of a controllable but realistic gold-standard, that can also be used to generate data on a clinical scanner. Vegetables with fibrous structures such as asparagus have been used [2] as well as prepared rat spinal cords [3] or other animal nerve structures i.e. garfish or lobster nerves [4]. However, these studies lack the possibility to construct custom geometries; most importantly, interdigitated crossing fibers cannot be constructed. Finally, the properties of such phantoms might change over time.

Therefore, hardware phantoms made from capillaries or artificial fibers have proven to be an extremely valuable tool. Since glass capillaries e.g. $[5,6]$ are rigid and PTFE (Teflon $R$ ) [5] capillaries have a large wall thickness (300 $\mu \mathrm{m})$, they impose limits to the geometry of phantom design. In contrast to capilaries, 
artificial fiber phantoms $[7,8,9,10,11]$ are more adaptable to the geometry and configuration needed for validation and testing. Hydrophobic fiber materials (e.g. Dyneema $(\mathbb{R}$, polyamide) have desirable properties, because fractional anisotropy (FA) is reported to be higher than in hydrophilic materials such as viscose, linen and hemp [10].

Recently, a phantom was developed [11] using acrylic fibers packed at 1900 fibers $/ \mathrm{mm}^{2}$. The authors report a signal-to-noise ratio (SNR) greater than 4 at $b$-values up to $10,000 \mathrm{~s} / \mathrm{mm}^{2}$; however, the employed voxel volume was greater dan $1400 \mathrm{~mm}^{3}$, which is far beyond the voxel size of approx $8 \mathrm{~mm}^{3}$ used in clinical practice. Furthermore, fiber packing density achieved by enclosing fibers tightly in a tube has an important effect on diffusivity and anisotropy measured in the phantom $[8,9]$. Shrink-wrapping fibers can increases anisotropy up to maximum FA values of 0.4 and 0.6 respectively $[8,9]$, compared to a FA of 0.2 [7] or 0.3-0.4 [11] in a non shrink-wrapped phantom.

Here, the aim is to construct and test a phantom suitable for data acquisition using a clinical protocol, with a $T_{2}$ and anisotropy values similar to human white matter, by packing fiber bundles tightly in heat shrink tubes. The phantoms presented in this paper are suitable for validating diffusion models and fiber tracking algorithms, since complex, sub-voxel interdigitated fiber bundles, representing crossing, kissing or combined geometries are possible.

First a preliminary study was performed to investigate the effect of fiber packing density on diffusion anisotropy. Based on the results, crossing phantoms were built with fiber organization similar to white matter architecture, by crossing a larger number of small diameter $(<0.4 \mathrm{~mm})$ fiber bundles. The phantoms were specifically constructed to produce intra-voxel crossings in an interdigitating fashion. We were able to perform DTI based fiber tracking and to fit nongaussian diffusion models on the phantom data.

\subsection{METHODS}

\subsubsection{Determination of optimal yarn packing density}

Fiber packing density $\rho_{f}$ is crucial for optimal phantom design, since it determines the amount of anisotropic diffusion that can be established. Secondly, the water content inside the phantom needs to be sufficient in order to get enough signal.

The experiments were performed at room temperature $\left(21-23^{\circ} \mathrm{C}\right)$. It should be considered that at higher (body) temperature, and at a given fiber packing density, diffusion parameters might differ slightly, for instance ADC and FA are expected to be slightly increased at higher temperatures, as demonstrated in a temperature-controlled diffusion phantom [12]. Therefore, we have optimized fiber packing density for usage at room temperature. Optimal fiber density is 
assessed by evaluating the diffusive properties -parallel versus perpendicular diffusion, and FA- of tubular phantoms while varying yarn packing density inside the phantoms.

\subsubsection{Phantom construction}

STRAIGHT PHANTOMS A tubular phantom is built by wrapping a bundle of yarns in shrink wrap tubing (Farnell InOne, NL). The yarn material used is Kuag Diolen $^{\mathrm{TM}_{22}}$ dtex f 18 (KUAG Elana $\mathrm{GmbH}$, Heinsberg-Oberbruch, Germany), a polyester yarn. Each yarn strand is composed of 18 filaments ("fibers") with a circular diameter of $10 \mu \mathrm{m}$. Phantom construction consists of three stages:

1. Winding a number of yarns to a bundle. A reel driven by a drill operating at $\approx 600 \mathrm{rpm}$ is used to wind yarns. The revolutions of the drill are counted by a switch connected to a PC running custom counting software.

2. Once an appropriate amount of yarns is wound, the bundle is taken off the reel and transferred to water or an aqueous solution

3. A piece of shrink tube is slid over the bundle, and the whole is transferred to a >95 degrees Celsius water/solution bath. The phantom is left there for at least 5 mins to ensure maximum shrinkage of the shrink tube. 5 Straight phantoms were constructed with a range of $7,000-11,000$ yarns in a $14 \mathrm{~mm}$ -pre-shrinking diameter- tube.

Shrinking reduces the diameter of the tube to $4 \cdot 7-4.8 \mathrm{~mm}$, this leads to $(7200-$ 10,800 fibers $/ \mathrm{mm}^{2}$ ) after shrinking. The shrinking stage was performed in tap water or de-mineralized water doped with $0.03 \mathrm{~g} / 1 \mathrm{MnCl}_{2} \cdot 4 \mathrm{H}_{2} \mathrm{O}$ to adjust $T_{2}$ to a value comparable to human white matter $T_{2}$ and $2.4 \mathrm{~g} / 1 \mathrm{NaCl}$ for resistive coil loading.

Phantoms were kept in their respective solution during the entire experiment. The results of the optimal fiber density experiment can be found in the Results section. Combining the findings of the anisotropy ratio and the FA and ADC values, we choose a yarn density of 10,000 yarns $\left(\approx 470\right.$ yarns $/ \mathrm{mm}^{2}$ or $8.5 \cdot 10^{3}$ fibers $/ \mathrm{mm}^{2}$ ) to be optimal.

CONSTRUCtion of CROSSing Phantoms A crossing " $X$ " shaped phantom is constructed by interdigitating smaller bundles, until the required yarn density is reached: The process is shown schematically in figure 2.1. Based on the results in the straight phantoms, crossing phantoms consist of 10,000 yarns per leg. 25 Bundles of 400 yarns ( 7200 fibers) were interdigitated to form crossings. Together, these o. $4 \mathrm{~mm}$ thick bundles account for a phantom leg diameter of $5.7 \pm 0.9 \mathrm{~mm}$ and a fiber density of $8.5 \cdot 10^{3}$ fibers $/ \mathrm{mm}^{2}$. The legs of the " $X$ " shape were fixed at 30,50 and 65 degrees respectively. The $14 \mathrm{~mm}$ shrink-wrap tube was slid over 

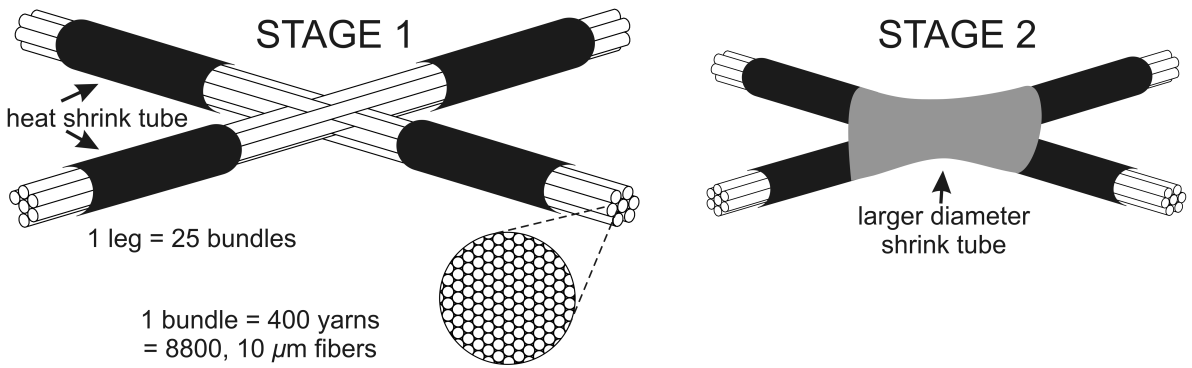

Figure 2.1: Schematics of crossing phantom construction. Stage 1: Bundles of yarns are placed interdigitating on top of each other. The legs of the âĂIXâĂİ are wrapped with a shrink-wrap tube. The tube is slid over the fibers up to the crossing, leaving only a short portion of the crossing fibers uncovered, and put into a heated water bath. Stage 2: the center of the $X$ is covered by another piece of shrink wrap tube and heated again.

the legs tightly up to the crossing area and was shrunk over the legs and a piece of $20 \mathrm{~mm}$ tube was shrunk over the center of the crossing. The crossings have dimensions (width $\times$ height) of $9.3 \pm 0.2$ by $10.1 \pm 0.2 \mathrm{~mm}$. All phantoms were fixated in the container filled with $0.03 \mathrm{~g} / 1 \mathrm{MnCl}_{2} \cdot 4 \mathrm{H}_{2} \mathrm{O}$ and $2.4 \mathrm{~g} / 1 \mathrm{NaCl}$, see figure 2.2.

DATA ACQUiSition The phantoms were aligned parallel to the bore (z-axis) of a 3 T Siemens Allegra scanner (Siemens, Erlangen, Germany) equipped with a standard birdcage head coil; maximum gradient strength $40 \mathrm{mT} / \mathrm{m}$. Experiments were done at room temperature $21-23^{\circ} \mathrm{C}$.

The experiments below were performed on the tubular phantoms. A standard twice refocused spin-echo echo-planar imaging diffusion-weighted sequence was used. In all measurements, 50 slices with $2 \times 2 \times 2 \mathrm{~mm}$ voxels in a $128 \times 128 \mathrm{ma}-$ trix were acquired, which are clinically relevant acquisition parameters. DW-MRI was performed in 3 orthogonal directions (slice, read-out and phase-encoding directions) plus a $b_{0}$ image, with $b$ varying from $250-3000 \mathrm{~s} / \mathrm{mm}^{2}$. TR/TE was kept constant across measurements at 8700/104 ms. DW-MRI with 48 diffusion directions $+6 b_{0}$ volumes (interleaved at every 12 diffusion volumes) at $b=1000$ $\mathrm{s} / \mathrm{mm}^{2}$ was acquired. 50 slices, $2 \times 2 \times 2 \mathrm{~mm}$ voxels, $128 \times 128$ matrix.

The crossing phantoms were scanned with 54 and 132 diffusion directions schemes, derived from [13]. A $b$-value of $1000 \mathrm{~s} / \mathrm{mm}^{2}, \mathrm{TR} / \mathrm{TE}=6300 / 83 \mathrm{~ms}$. $\Delta=32.44 \mathrm{~ms}, \delta=25.34 \mathrm{~ms}$, was applied for standard DTI. SNR is 11.9 in the $b_{0}$ image, and 5.0 in the diffusion weighted image (estimated from a VOI in the leg of the $50 \mathrm{deg}$ phantom). A b-value of $3000 \mathrm{~s} / \mathrm{mm}^{2}$, TR/TE $7400 / 105 \mathrm{~ms}$. $\Delta=43.44 \mathrm{~ms}, \delta=36.34 \mathrm{~ms}$, was used to enable q-ball [14] and Diffusion Orientation Transform (DOT) [15] reconstructions. SNR is 2.0 in the diffusion weighted image. 


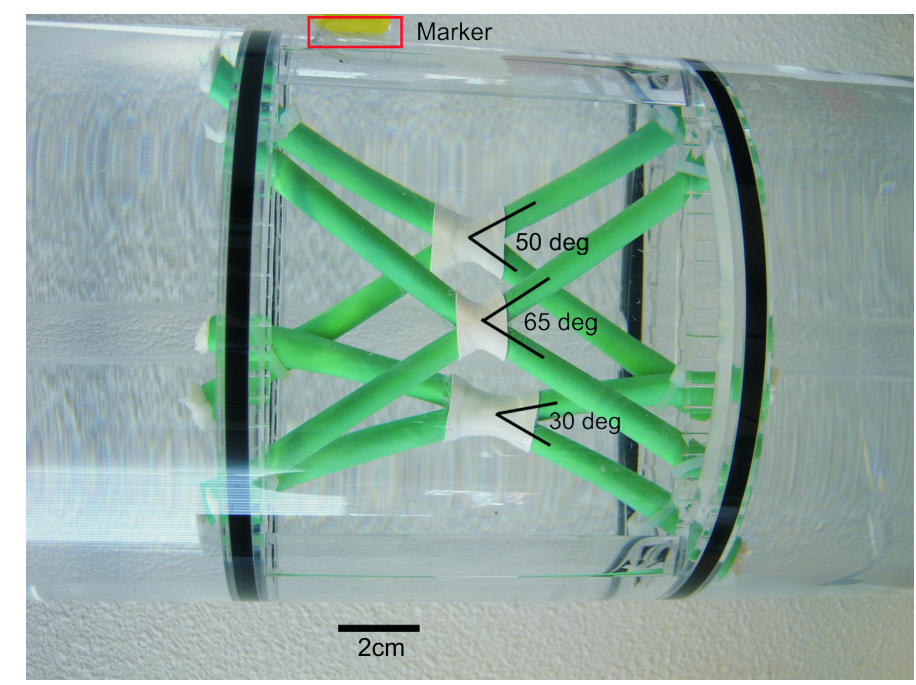

Figure 2.2: Overview of the crossing phantoms with the respective crossing angles. The container is a perspex cylinder and the phantoms are fixed with tie-wraps to prevent vibration artefacts.

DATA PROCESSING The anisotropy in each representative voxel of the tubular phantoms was computed by calculating the parallel and perpendicular diffusion; anisotropy $=\mathrm{ADC}_{\|} / \mathrm{ADC}_{\perp}$. Since the long axis of the phantoms is aligned to the $\mathrm{z}$-axis of the scanner, this is the parallel signal.

Tensor calculation and computation of FA/ADC values was done in BrainVoyagerQX 1.9.10 (Brain Innovation, NL) and Matlab R2007b (The Mathworks, USA), together with a in house developed toolbox (available from http://support. brainvoyager. com/available-tools/).

Fiber tracking was performed with a streamline algorithm using a local projection operator, as proposed by [16], implemented in-house. The tracing was terminated in regions were FA $<$ o.2. Fiber tracking was also terminated when the angle between the current and the previous tracing step was smaller than 40 degrees. The linear measure shape $C_{l}$ as introduced in [16]

$$
C_{l}=\frac{\lambda_{1}-\lambda_{2}}{\sqrt{\lambda_{1}^{2}+\lambda_{2}^{2}+\lambda_{3}^{2}}}
$$

with $\lambda_{1}>\lambda_{2}>\lambda_{3}$ the three eigenvectors of the diffusion tensor, was also computed. In regions of kissing or crossing fibers it is expected that $C_{l}$ is reduced with respect to the value of $C_{l}$ in coherent fiber bundles [17]. Q-ball and Diffusion Orientation Transform (DOT) reconstructions were done in DTItool vi.o [BIOMIM, Eindhoven University of Technology, NL]. Q-ball reconstruction was performed with 6th order spherical harmonics and a Laplace-Beltrami smoothing factor of 0.0006 [18]. The DOT reconstructions were made with 6th order spherical harmonics and $t=25 \mathrm{~ms}, R_{0}=20 \mu \mathrm{m}$. 

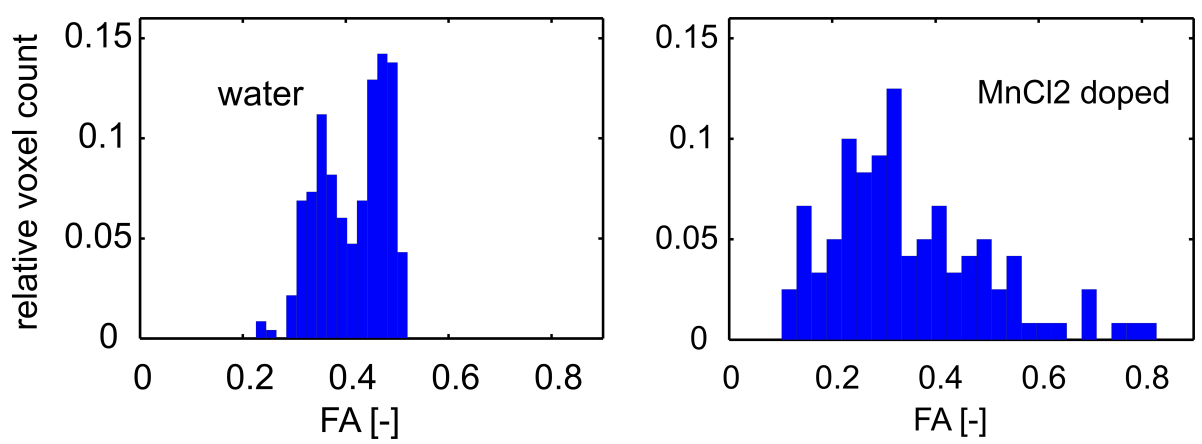

Figure 2.3: FA distributions of water and $\mathrm{MnCl}_{2} \cdot 4 \mathrm{H}_{2} \mathrm{O}$ doped 10,000 yarn phantoms taken from representative voxels at $b=1000 \mathrm{~s} / \mathrm{mm}^{2}$ and 54 diffusion directions. The distributions have been normalized for easy comparison. a: FA distribution of the water-based 10,000 yarn phantom at a $b$-value of $1000 \mathrm{~s} / \mathrm{mm}^{2}$. b: FA distribution of the $\mathrm{MnCl}_{2} \cdot 4 \mathrm{H}_{2} \mathrm{O}$ doped 10,000 yarn phantom at a $b$ value of $1000 \mathrm{~s} / \mathrm{mm}^{2}$. The distribution of water-based phantoms is restricted to a more narrow range than the distribution of the $\mathrm{MnCl}_{2}$ doped phantoms, the latter being more close to the range in human white matter FA distribution.

\subsection{RESULTS}

\subsubsection{Fiber packing density experiment}

Results are taken from representative, selected voxels inside phantoms. Across yarn densities, the anisotropy is relatively stable, ranging from 2.58-3.25 in the $\mathrm{MnCl}_{2}$ doped phantoms, see Table 2.1. On average, across increasing $b$-values and doped with $\mathrm{MnCl}_{2} \cdot 4 \mathrm{H}_{2} \mathrm{O}, 10$,ooo yarns offer the highest most stable anisotropy ratio of 2.9 .

Mean ADC (trace(D)/3) values $\left(0.376-0.491 \cdot 10^{-3} \mathrm{~mm}^{2} / \mathrm{s}\right)$ are in the range of, but slightly higher than, mean ADC values found in healthy human white matter, which is reported to be $0.27-0.33 \cdot 10^{-3} \mathrm{~mm}^{2} / \mathrm{s}$ [19] at a $b$-value of $1000 \mathrm{~s} / \mathrm{mm}^{2}$. Mean FA values (0.272-0.361, with maximum FA up to o.6-0.8; see Fig. 2.3 and Table 2.2) are in accordance with FA values found in large parts of human white matter. As can be seen in Table 2.2, a lower FA is observed for the phantoms with 7,8 or 11,000 yarns as compared to the phantoms containing 9,000 and 10,000 yarns. The packing density might be too low in the $7-8,000$ yarns phantoms, while water content is probably too low in the 11,000 yarns phantom, possibly causing a lower FA. A difference in ADC value is observed between $\mathrm{MnCl}_{2}$ doped and water only phantoms. This might be due to the presence of paramagnetic manganese ions, which cause the $T_{2}$ to be lowered and also decrease the apparent diffusion constant, as demonstrated in [20]. The relatively large standard deviation of FA values may be attributed to measurement noise, and potential partial volume effects in some voxels on the border between phantom tube and water. 


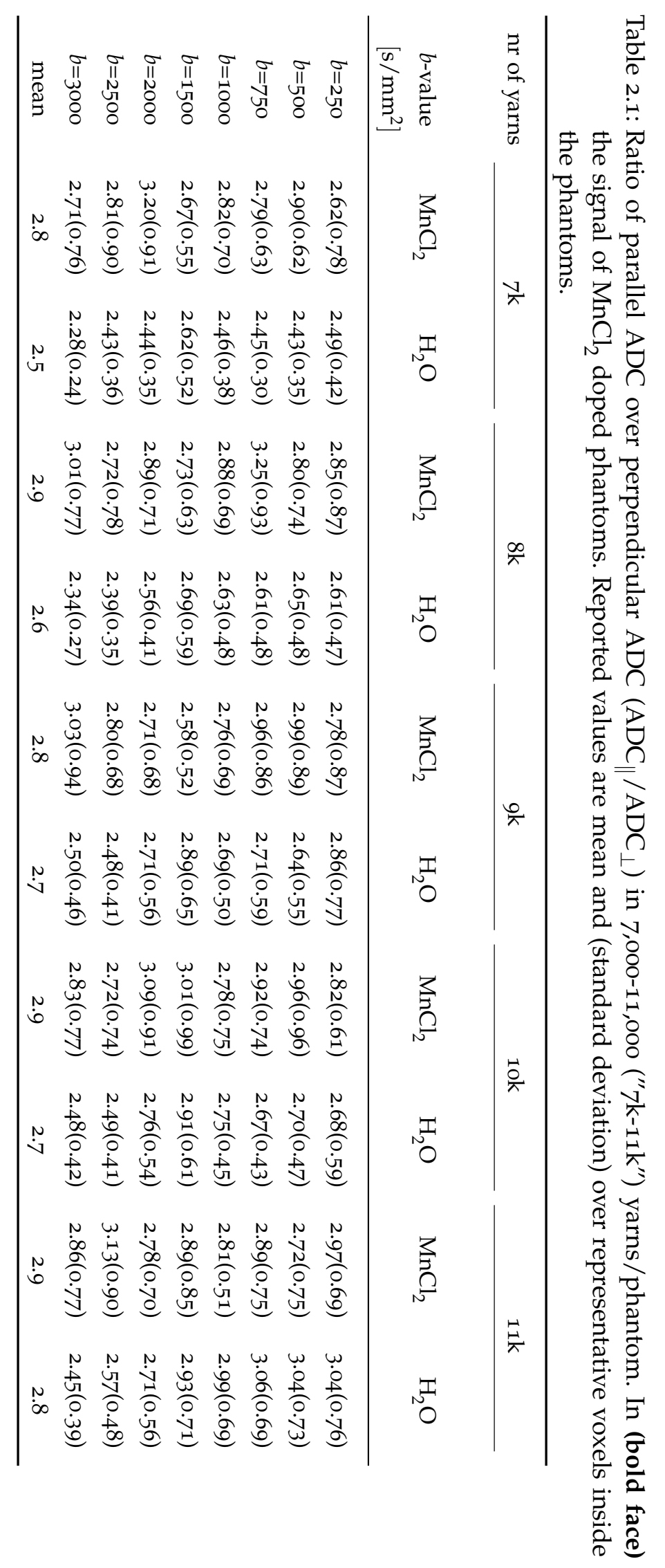


Table 2.2: Mean(standard deviation) of FA and mean ADC $\left[10^{-3} \mathrm{~mm} / \mathrm{s}^{2}\right]$ values of straight phantoms with 7,000-11,000 ("7k-11k") yarns/phantom at $b=1000\left[\mathrm{~s} / \mathrm{mm}^{2}\right], 48$ diffusion directions, $6 b_{0} \mathrm{~s}$.

\begin{tabular}{ccccccc}
\hline \multicolumn{2}{c}{ Nr of yarns } & $7 \mathrm{k}$ & $8 \mathrm{k}$ & $9 \mathrm{k}$ & $10 \mathrm{k}$ & $11 \mathrm{k}$ \\
\hline water & $F A$ & $0.345(0.04)$ & $0.345(0.06)$ & $0.401(0.06)$ & $0.414(0.07)$ & $0.346(0.16)$ \\
water & $A D C$ & $0.482(0.17)$ & $0.474(0.16)$ & $0.434(0.14)$ & $0.449(0.14)$ & $0.478(0.15)$ \\
$\mathrm{MnCl}_{2}$ & $F A$ & $0.272(0.14)$ & $0.293(0.10)$ & $0.361(0.18)$ & $0.347(0.15)$ & $0.335(0.11)$ \\
$\mathrm{MnCl}_{2}$ & $A D C$ & $0.491(0.10)$ & $0.406(0.11)$ & $0.409(0.15)$ & $0.376(0.13)$ & $0.383(0.10)$ \\
\hline
\end{tabular}

\subsubsection{Diffusion Tensor estimation}

In Fig. 2.4a the tensor boxoids are shown of 50 degree crossing phantom. It reflects the true 3-dimensional crossing, unlike the sheet-like crossings constructed by stacking layers of capillaries. Boxoids have a more elongated shape in the legs of the " $X$ " and the dominant direction of the diffusion tensor is along the direction of the yarn-fibers, while a more cubic shape is observed in the crossing region of the phantoms, indicating lower modelled diffusion anisotropy and showing the known inability of the diffusion tensor to model the multimodal diffusion profile. a detail, is shown. In Fig. 2.4b, The linear shape measure $C_{l}$ is shown. Higher values of $C_{l}$, indicating an increase in the linear shape of the tensor ellipsoid, are observed in the legs of the phantom, while a decrease in $C_{l}$, common for crossing, kissing or diverging fibers, is observed in the crossing of the phantom.

\subsubsection{DTI Fiber tracking}

ROIs were placed in the legs of the phantoms at slices 10 and 40 (of total 50). Seed density was adjusted empirically to $7 \times 7$ seeds per voxel in-plane. Results are shown in figure 2.5 for the most challenging case, the 30 degree crossing. As can clearly be observed in figure 2.5a (FA threshold o.2), all tracked fibers stay on the same side, while the true fibers cross. When applying a forced AND operation, like commonly used in fiber tracking studies [21], and lowering the FA threshold (FA>0.05), tracked fibers cross (Fig. 2.5b), though with a seemingly unrealistic curved path through the crossing.

\subsubsection{Q-ball and DOT reconstructions}

Q-ball and DOT reconstructions were made from the data set acquired with 132 directions at a b-value of $3000 \mathrm{~s} / \mathrm{mm}^{2}$. Fig. 2.6 is an example of the potential 

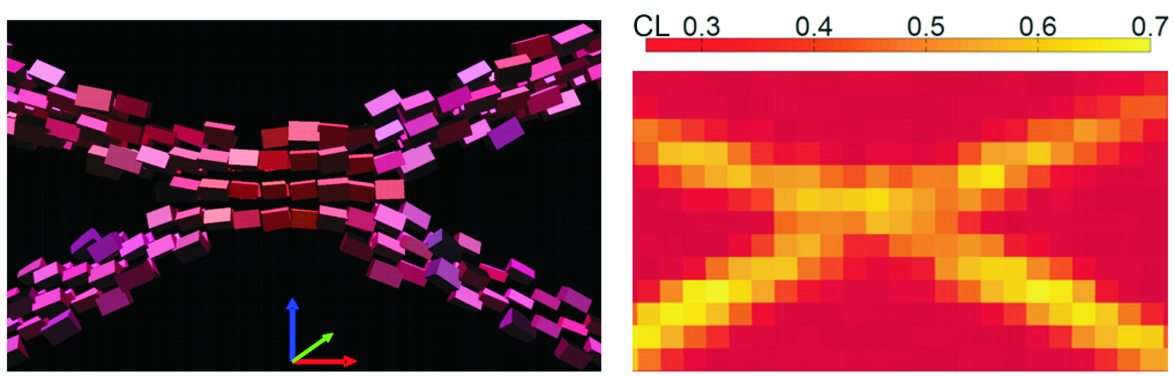

Figure 2.4: Tensor boxoids of the crossing phantom at $b=1000 \mathrm{~mm}^{2} / \mathrm{s}, 54$ diffusion directions. a: Lateral view of the 50 degree phantom. Direction color-coded boxoids (red: left-right, blue: inferior superior, green: anterior-posterior) in the 50 degree fiber crossing phantom. The color coding depends on the orientation of the phantom in the scanner, which is: longest axis in the direction of the Bo field in the current setup. The cuboids show a more cubic shape where fibers cross. This indicates decreased anisotropy common for crossing voxels. More elongated boxoids are observed in the areas where fibers run parallel, indicating increased anisotropy in that area. b: Map of linear shape measure $C_{l}$ of the 50 degree crossing phantom, showing higher values (yellow colors) in the legs of the phantom, and lower values (red colors) in the crossing region. This confirms the observation of a difference in shape in the boxoids in the crossing (lower $C_{l}$ ) versus the areas where fibers run in parallel (higher $C_{l}$ ).
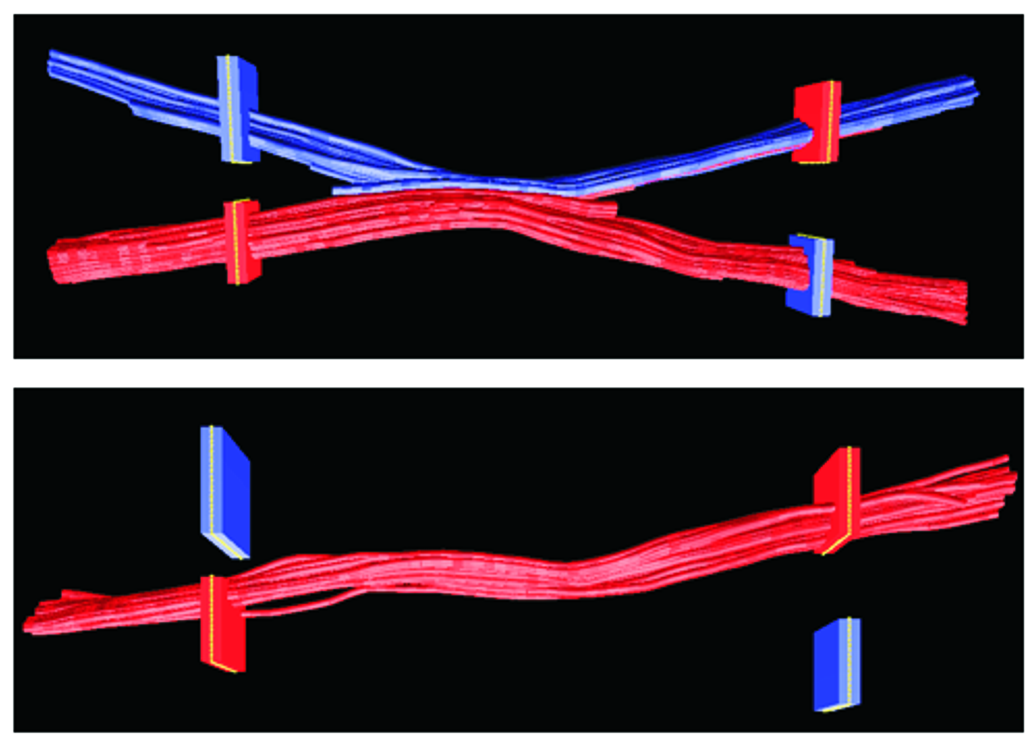

Figure 2.5: Examples of fiber tracking on the 30 degree phantom. Data set used: 54 diffusion directions, $b=1000 \mathrm{~s} / \mathrm{mm}^{2}$. a: Fibers tracked from both ROIs on the left with FA $>0.2$ threshold. Phantom fibers are running contralateral, but the tracked reconstructed fibers stay ipsilateral. This behavior was observed in all three phantoms. b: Fibers forced contra-laterally by tracking with an AND operation and low FA $>0.05$ threshold. 

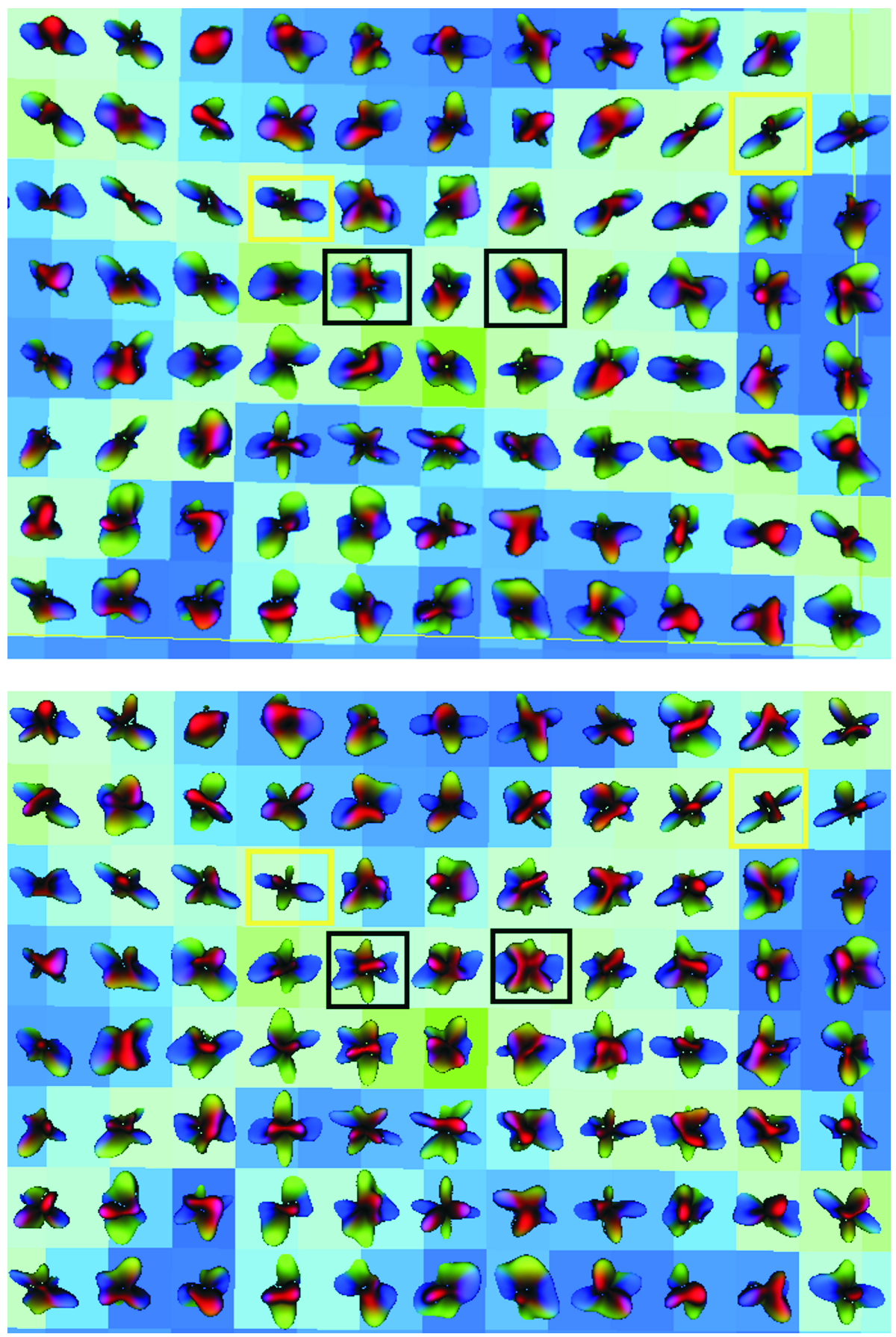

Figure 2.6: Q-ball and DOT reconstructions of the 65 degree crossing phantom. The reconstructions are RGB color coded and overlayed on a FA map. (top): Q-ball reconstruction of the 65 degree crossing phantom at $b=3000 \mathrm{~s} / \mathrm{mm}^{2}, 132$ diffusion directions. Yellow boxes show examples of a single-fiber ODF. In boxes enclosed with black boxes, examples of multiple fiber ODFs. (bottom): DOT reconstruction of the 65 degree crossing phantom at $b=3000 \mathrm{~s} / \mathrm{mm}^{2}$, 132 diffusion directions. DOT parameters: $t=25 \mathrm{~ms}, R_{0}=15 \mu \mathrm{m}$. In yellow boxes examples of a single-fiber probability profile. In black enclosed boxes, examples of multiple fiber probability profiles. 
use of the phantom for comparison of HARDI methods and validation of their improvement of the diffusion tensor in modelling multi-modal diffusion profiles. In Fig. 2.6a the normalized Q-ball reconstructions are shown in RGB color coding, overlaid on the FA map. The " $\mathrm{X}$ " shape of the phantom is clearly recognizable. In the legs of the phantom, where fibers are arranged in parallel, a single-lobe ODF is observed (yellow boxes). The center of the phantom contains multi-modal ODFs consisting of more than one orientation in the black boxes. In Fig. 2.6b, the DOT reconstructions of the data are shown. Again, we observe single lobe ODFs in the legs of the phantom and multiple lobe-ODFs in the center of the phantom containing the crossing fibers.

\subsection{DISCUSSION}

In this paper we have presented a phantom that can be used with clinical MR protocols, suitable for validation of a large spectrum of DW-MRI applications, from acquisition to fiber tracking and with realistic white matter (WM) properties in terms of relaxivity and diffusive properties.

Previous studies [9, 10], where a similar phantom design was used, did not consider yarn packing density. It was shown with Monte Carlo simulations that FA is positively related to yarn packing density [8]. In our experiment, optimal yarn packing density was empirically determined and optimized by FA and the anisotropy ratio $A D C_{\|} / A D C_{\perp}$. The yarn density of approx. $8.5 \cdot 10^{3}$ fibers $/ \mathrm{mm}^{2}$ was found to be optimal. The resulting anisotropy ratio was 2.9, close to the ratio of 2.8 found in the non-myelinated walking leg nerve of the lobster at a $b$-value of $1000 \mathrm{~s} / \mathrm{mm}^{2}$ [4], and a FA distribution close to the distribution found in human white matter [19] producing an average FA of 0.35, with maximum FA of $0.6-0.8$ in several voxels. It should be noted that, since the fibers are hydrophobic, only intercellular diffusion is modeled with these types of phantom materials. $T_{2}$ relaxation of the phantom is adapted closely to $W M T_{2}$ by doping it with a $\mathrm{MnCl}_{2} \cdot 4 \mathrm{H}_{2} \mathrm{O}$ solution. $T_{1}$ relaxation is not included in the present study, because DW-MRI is a technique mainly based on $T_{2}$ contrast.

Streamline fiber tracking on a 54 direction DTI scan with a $b$-value of 1000 $\mathrm{s} / \mathrm{mm}^{2}$ is feasible on the crossing phantoms and shows the incapability of DTI to resolve crossing fibers DOT and Q-ball reconstruction of the diffusion profiles acquired at $b=3000$ and 132 diffusion directions reveal multi-modal diffusion profiles in voxels containing crossing yarn strands. A clear difference can be observed between voxels containing multiple fiber orientation and voxels containing a single fiber orientation.

The presented phantoms provide a platform for highly purpose-adaptable phantoms. The phantom in itself is flexible and may be bent into a circular or helical configuration. The fibers inside the phantom may be twisted, crossed or kissed to create more challenging data sets to evaluate and improve fiber track- 
ing algorithms. Other possible applications include: comparison and optimization of acquisition schemes (e.g. diffusion direction gradient schemes, $b$-values, q-space sampling), quality control in multi-center studies, and validation and comparison of non-Gaussian diffusion models.

The raw data from DW-MRI scans will be made available to the community at http://www. brainvoyager. com/diffusionphantoms/.

\section{ACKNOWLEDGEMENTS}

PP would like to thank Erik van den Bergh (Siemens Healthcare, NL) for initial project ideas; Sven Gijsen (Maastricht Brain Imaging Center, NL) for the custom counting software; Marjanne Markerink and Hellen Steinbusch (School for Mental Health and Neuroscience, Maastricht University, NL) for use of the lab facilities.

\section{REFERENCES}

[1] A Leemans, J Sijbers, M Verhoye, A Van Der Linden, and D Van Dyck. Mathematical framework for simulating diffusion tensor MR neural fiber bundles. Magn Reson Med, 53(4):944-53, 2005.

[2] S Boujraf, R Luypaert, H Eisendrath, and M Osteaux. Echo planar magnetic resonance imaging of anisotropic diffusion in asparagus stems. Magnetic Resonance Materials in Biology, Physics, and Medicine, 13:82-90, 2001.

[3] JSW Campbell, K Siddiqi, VV Rymar, AF Sadikot, and GB Pike. Flow-based fiber tracking with diffusion tensor and q-ball data: Validation and comparison to principal diffusion direction techniques. NeuroImage, 27:725-736, 2005.

[4] C Beaulieu. The basis of anisotropic water diffusion in the nervous system-a technical review. NMR Biomed., 15(7-8):435-455, 2002.

[5] CP Lin, VJ Wedeen, JH Chen, C Yao, and WY Tseng. Validation of diffusion spectrum magnetic resonance imaging with manganese-enhanced rat optic tracts and ex vivo phantoms. Neuroimage, 19:482-495, 2003.

[6] N Yanasak and J Allison. Use of capillaries in the construction of an MRI phantom for the assessment of diffusion tensor imaging: demonstration of performance. Magn. Reson. Imaging, 24:1349-1361, 2006.

[7] M Perrin, C Poupon, B Rieul, P Leroux, A Constantinesco, JF Mangin, and D Le Bihan. Validation of q-ball imaging with a diffusion fibre-crossing phantom on a clinical scanner. Philos Trans R Soc Lond B Biol Sci, 36o:881$891,2005$. 
[8] E Fieremans, Y De Deene, S Delputte, MS Ozdemir, Y D'Asseler, J Vlassenbroeck, K Deblaere, E Achten, and I Lemahieu. Simulation and experimental verification of the diffusion in an anisotropic fiber phantom. Journal of Magnetic Resonance, 190:189-99, 2008.

[9] M Watanabe, S Aoki, Y Masutani, O Abe, N Hayashi, T Masumoto, H Mori, $\mathrm{H}$ Kabasawa, and K Ohtomo. Flexible ex vivo phantoms for validation of diffusion tensor tractography on a clinical scanner. Radiat. Med., 24(9):605609, 2006.

[10] R. Lorenz, M. E. Bellemann, J. Hennig, and K. A. Il'yasov. Anisotropic Phantoms for Quantitative Diffusion Tensor Imaging and Fiber-Tracking Validation. Applied Magnetic Resonance, 33(4):419-429, May 2008.

[11] C Poupon, B Rieul, I Kezele, M Perrin, F Poupon, and J-F Mangin. New Diffusion Phantoms Dedicated to the Study and Validation of High-AngularResolution Diffusion Imaging ( HARDI ) Models. Magnetic Resonance in Medicine, 1283(October 2007):1276-1283, 2008.

[12] C Reischauer, P Staempfli, T Jaermann, and Ps Boesiger. Construction of a Temperature-Controlled Diffusion Phantom for Quality Control of Diffusion Measurements. Journal of Magnetic Resonance Imaging, 698:692- 698, 2009 .

[13] DK Jones, MA Horsfield, and A Simmons. Optimal strategies for measuring diffusion in anisotropic systems by magnetic resonance imaging. Magn. Reson. Med., 42:515-525, 1999.

[14] D Tuch. Q-ball imaging. Magn. Reson. Med., 52:1358-1372, 2004.

[15] E Ozarslan, TM Shepherd, BC Vemuri, SJ Blackband, and TH Mareci. Resolution of complex tissue microarchitecture using the diffusion orientation transform (DOT). Neuroimage, 31:1086-1103, 2006.

[16] C Westin, SE Maier, H Mamata, A Nabavi, FA Jolesz, and R Kikinis. Processing and visualization for diffusion tensor MRI. Medical Image Analysis, 6:93-108, 2002.

[17] A Roebroeck, R Galuske, E Formisano, O Chiry, H Bratzke, I Ronen, D-S Kim, and R Goebel. High-resolution diffusion tensor imaging and tractography of the human optic chiasm at 9.4T. NeuroImage, 39:157-168, 2008.

[18] M Descoteaux, E Angelino, S Fitzgibbons, and R Deriche. Apparent diffusion coefficients from high angular resolution diffusion imaging: Estimation and applications. Mag Reson Med, 56:395-410, 2006.

[19] S Mori. Introduction to Diffusion Tensor Imaging. Elsevier, 2007. 
[20] J Zhong, RP Kennan, and JC Gore. Effects of Susceptibility Variations on NMR Measurements of Diffusion. J Magn Reson, 95:267-280, 1991.

[21] S Wakana, H Jiang, LM Nagae-Poetscher, and S Mori. Fiber tract-based atlas of human white matter anatomy. Radiology, 230:77-87, 2004. 

PHYSICAL ORIENTATION AFFECTS DIFFUSION MEASURES: A HARDWARE PHANTOM STUDY

this chapter is based on Pim Pullens, Alard Roebroeck, Matteo Bastiani, Rainer Goebel and Kâmil Uludağ. Physical orientation affects diffusion measures: a hardware phantom study. In preparation 


\section{ABSTRACT}

It was shown recently in an isotropic water phantom that ADC values are not equal in each measured diffusion direction due to gradient mis-calibrations. MR resonance frequency is also dependent on the orientation of anisotropic structures in the magnetic field, which possibly contributes to deviations in the diffusion measurement.

The effect of orientation of an anisotropic diffusion phantom on the non-DW (b0) signal, FA and the first eigenvalue $\left(\lambda_{1}\right)$ was investigated. The results show considerable variations in $b 0$, FA and $\lambda_{1}$ in the anisotropic phantom at different orientations in the magnetic field. The free water $\lambda_{1}$ in the phantom is not affected by the rotation. The observed $b 0$, FA and $\lambda_{1}$ signal changes might therefore originate from susceptibility changes due orientation change alone as demonstrated earlier in bone and white matter with gradient-echo imaging. Gradient mis-calibration likely adds to this effect as ADC is affected by miscalibrated gradients.

Further studies including Monte-Carlo simultations are needed to investigate the source of the variations. Our results consequently indicate that tissue characterization with DW-MRI in tissue with varying fiber structure orientation (e.g. white matter) will probably be affected by this phenomenon.

\subsection{INTRODUCTION}

The diffusion tensor and derived measures obtained from a diffusion-weighted MRI (DW-MRI) experiment are assumed to be rotationally invariant with respect to fiber orientation in the scanner's reference frame [1]. In DW-MRI studies using multiple subjects, where equal positioning of the subjects is not guaranteed, researchers have to rely on diffusion measures (such as tensor eigenvalues, fractional anisotropy (FA) and mean diffusivity (MD)) being independent of orientation of the subject in the scanner. Consequently, in any anisotropic object we would expect diffusion anisotropy values that are similar independent of the orientation of the object, provided that a sufficient amount of unique gradient directions is used [2].

The measured apparent diffusion constant $D_{\text {app }}$ is likely to be different from the real diffusion constant $D$ if there is a difference between $T_{2}$ caused by molecular interactions and $T_{2}^{*}$ caused by local susceptibility changes [3]. Magnetic susceptibility, as can be characterized by examining $T_{2}^{*}$, changes with the orientation of anisotropic structures in the main magnetic field [4]. It was demonstrated in a recent paper [5], that white matter (WM) fiber bundles oriented along the main magnetic field $\left(B_{0}\right)$ have lower relaxation rates $\left(R_{2}^{*}\right.$ or $\left.1 / T_{2}^{*}\right)$ than WM fibers running perpendicular to $\mathrm{B}_{0}$. The authors also investigated the relation between $R_{2}^{*}$ 


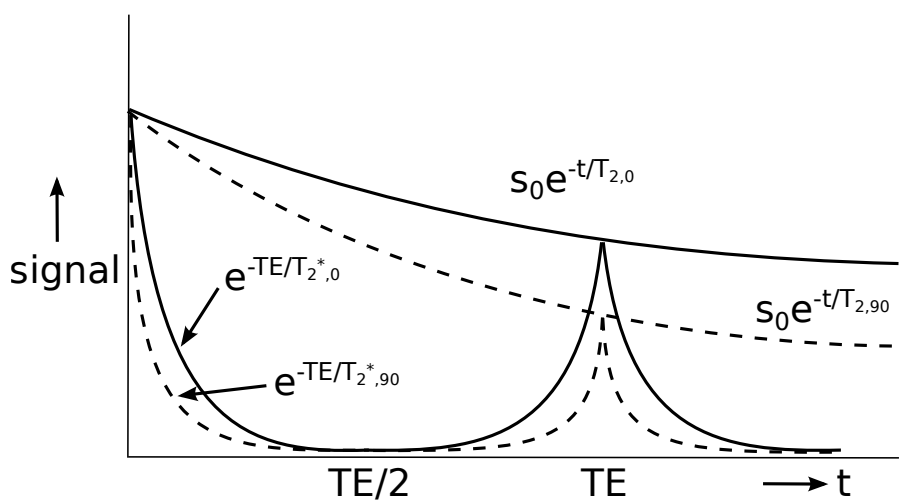

Figure 3.1: Effect of an orientation dependent change in $T_{2}^{*}$ on the spin-echo signal decay (o, solid line: phantom parallel to $z$-axis; 90, dashed line: phantom perpendicular to the $z$-axis). An increase in $T_{2}^{*}$ will cause the signal to decrease faster, thereby decreasing $T_{2}$.

and FA and could not find a significant relation between $R_{2}^{*}$ and FA. However, in some tracts a positive but no significant relation was found.

This gives rise to the question if there exists a relation between orientation of an anisotropic structure and diffusion measures. In this paper, we explored the effect of orientation of an anisotropic diffusion phantom on the DW-MRI signal without diffusion weighting ( $b 0$ signal), FA and MD values. The use of a phantom enables us to induce orientation-dependent susceptibility changes and sample diffusion parameters (diffusion tensor and derived measures) at different orientations. Because the phantom contains anisotropic structures in three orientations, it enables us to compare diffusion parameters in perpendicular oriented tubes in one measurement.

\subsection{THEORY}

In the presence of a susceptibility change in a spin-echo acquisition, $T_{2}^{*}$ will be different and thereby affecting the $T_{2}$ value in the sample. The effect is demonstrated in figure 3.1. The measured signal $S$ is a function of the diffusion constant, $f(D)$, and the orientation-dependent susceptibility effect $T_{2, \theta}^{*}$. The signal $S_{1}, S_{2}$ with the phantom aligned parallel and at an angle $\theta$ to the $z$-axis respectively is then given by

$$
\begin{aligned}
& S_{1}=f(D) \cdot e^{-T E / T_{2,0}^{*}} \\
& S_{2}=f(D) \cdot e^{-T E / T_{2, \theta}^{*}} \quad \theta \neq 0 .
\end{aligned}
$$


With $R_{2}^{*}=1 / T_{2}^{*}$, we can then describe the effect of susceptibility $\Delta R_{2}^{*}$ on the signal:

$$
\begin{aligned}
\frac{S_{1}}{S_{2}} & =e^{-T E\left(R_{2,0}^{*}-R_{2, \theta}^{*}\right)} \\
R_{2, \theta}^{*} & =R_{2,0}^{*}+\Delta R_{2}^{*} \\
\Delta R_{2}^{*} & =R_{2, \theta}^{*}-R_{2,0}^{*} \\
\frac{S_{1}}{S_{2}} & =e^{T E\left(\Delta R_{2}^{*}\right)}
\end{aligned}
$$

The effect of anisotropic structure orientation on magnetic susceptibility was demonstrated experimentally by rotating uniaxial trabechular bone in the magnetic field [4]. Local field inhomogeneities cause a change in the signal relaxation rate $R_{2}^{\prime}=1 / T_{2}^{\prime}$. Anisotropic structures aligned parallel to the main magnetic field $B_{0}$ show very little susceptibility effects (low $R_{2}^{\prime}$ ), while structures aligned perpendicular to $\mathrm{B}_{0}$ have a higher $R_{2}^{\prime}$. It was shown that there exists a relation between the rotation angle $\theta$ and $R_{2}^{\prime}$ [4]:

$$
R_{2}^{\prime} \propto \sin ^{2} \theta \text {. }
$$

Since the relaxation $R_{2}^{\prime}$ is related to $T_{2}^{*}$ via the following relation

$$
R_{2}^{*}=R_{2}+R_{2}^{\prime}=\frac{1}{T_{2}^{*}}=\frac{1}{T_{2}}+\frac{1}{T_{2}^{\prime}},
$$

it is sensible to investigate the $T_{2}^{*}$ effects in anisotropic structures. Expanding the work presented in [6], it was demonstrated in [7] that in the whole brain there is a strong $T_{2}^{*}$ effect depending on the orientation of white matter fiber orientation. Average $T_{2}^{*}$ was found to be lower in fibers oriented perpendicular to $B_{0}$, while in fibers oriented parallel to $\mathrm{B}_{0}$, a higher $T_{2}^{*}$ was found. A similar relation as earlier in [4] was found for white matter: $T_{2}^{*} \propto 1 / \sin ^{2} \theta$.

The effect of $\Delta R_{2}^{*}$ on the diffusion signal in a diffusion-weighted SE-EPI acquisition with multiple gradient directions is complex and hard to predict. For a PGSE experiment the effect of a background gradient on the signal can be described by $[3,8]$

$$
\begin{aligned}
\frac{S(\mathbf{G})}{S(0)} & =\exp \left[-(\gamma \delta G)^{2}(\Delta-\delta / 3) D\left(1+\frac{T E-\Delta / 2}{\delta G^{2}} \mathbf{G} \cdot \mathbf{G}_{0}\right)\right] \\
& =\exp \left[\left(-b_{a}+b_{c}\right) D\right],
\end{aligned}
$$

with $b_{a}$ the b-value, $b_{c}=\gamma^{2} \delta(\Delta-\delta / 3)(T E-\Delta / 2) \mathbf{G} \cdot \mathbf{G}_{0}$, and $\mathbf{G} \cdot \mathbf{G}_{\mathbf{0}}$ the dot product between diffusion-encoding and background gradient. In our case, the internal gradient is not constant, but dependent on the angle of rotation $\theta$ with the $z$-axis of the scanner, so equation 3.10 changes into

$$
\frac{S(\mathbf{G}, \theta)}{S(0, \theta)}=\exp \left[-\left(b_{a}-b_{c}(\theta)\right) D\right] \text {. }
$$


Equation 3.11 indicates that if the factor $b_{c}(\theta)$ is increased, the signal of faster moving spins is suppressed, increasing the weighting of the slower moving spins, resulting in a lower $D_{\text {app }}[3]$.

\subsection{METHODS}

\section{Anisotropic diffusion phantom}

A commercially available diffusion phantom (Brain Innovation BV, Maastricht NL [9]) containing nine anisotropic tubular structures in three planes was used in the experiments. The tubular phantoms are placed three near-orthogonal planes. Each tubular phantom consists of 10,000 tightly packed parallel yarns each consisting of 18 fibers ( 180,000 polyester fibers with a $10 \mu \mathrm{m}$ diameter) constrained by a shrink tube. A detailed description of phantom construction can by found in [10]. This type of phantom has a high degree of anisotropy (FA o.6-0.8) and can be scanned using a clinical DW-MRI protocol.

The nine tubular phantoms were fixed using tie-wraps on acrylic support rods in a spherical plastic container (diameter $16 \mathrm{~cm}$ ). The sphere was filled with demineralized water with $4 \mathrm{~g} / 1 \mathrm{NaCl}$ to ensure sufficient coil loading, $0.03 \mathrm{~g} / \mathrm{l}$ $\mathrm{MnCl}_{2} \cdot 4 \mathrm{H}_{2} \mathrm{O}$ to reduce $\mathrm{T}_{2}$ to around $80 \mathrm{~ms}$ and $0.5 \mathrm{~g} / 1 \mathrm{NaN}_{3}$ for conservation. The sphere was marked with points spaced every 45 degrees in each orthogonal plane. Figure 3.2 shows a photograph of the phantom and the positions of the marks used for positioning the phantom in the scanner.

\section{Acquisition}

The phantom was placed on a phantom holder (Siemens, Erlangen, D) which enables precise positioning in the iso-center. By aligning a grid point to a fixed point in the scanner we are able to accurately orient the phantom in the scanner. The phantom was rotated around the 3 scanner principal axes $x$ (in the $Y-Z$ plane), $y$ (in the $X-Z$ plane) and $z$ (in the $X-Y$ plane) in steps of 45 degrees. The $x$ axes corresponds with the left-right axis, the $y$ axis with the anterior-posterior and the $z$ axis with the inferior-superior axis in a human subject.

Figure 3.3 shows an example of the rotating procedure around one principal axis. At each orientation a DW-MRI scan was made with the following parameters: Siemens Allegra 3T birdcage single coil, double-refocussed SE-EPI diffusion sequence with FOV $192 \times 192 \mathrm{~mm}$, 70 slices, 2x2x2 mm voxel size. b-value 1000 $\mathrm{s} / \mathrm{mm}^{2}, 25$ diffusion directions and 360 images, with TR/TE $8000 / 79 \mathrm{~ms}$. The FOV was chosen as small as possible on our scanner to shorten the EPI readout. None of the diffusion directions is exactly aligned with any phantom orientation.

Slice positions were equal in each acquisition, and a preparation phase was performed before each acquisition. In total we obtained 24 different datasets. 


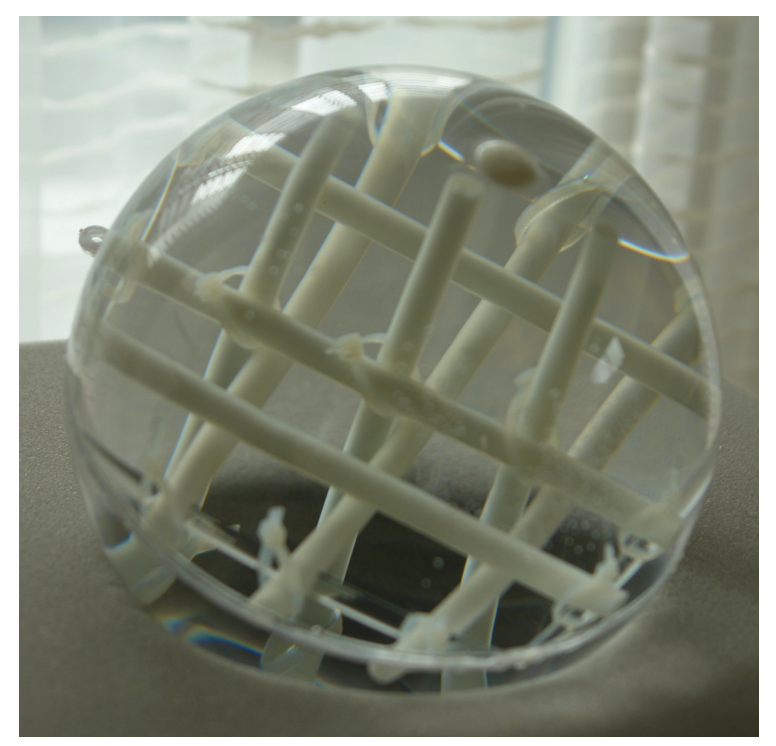

(a) Photograph of the phantom with 9 tubular phantoms in 3 near-orthogonal planes.

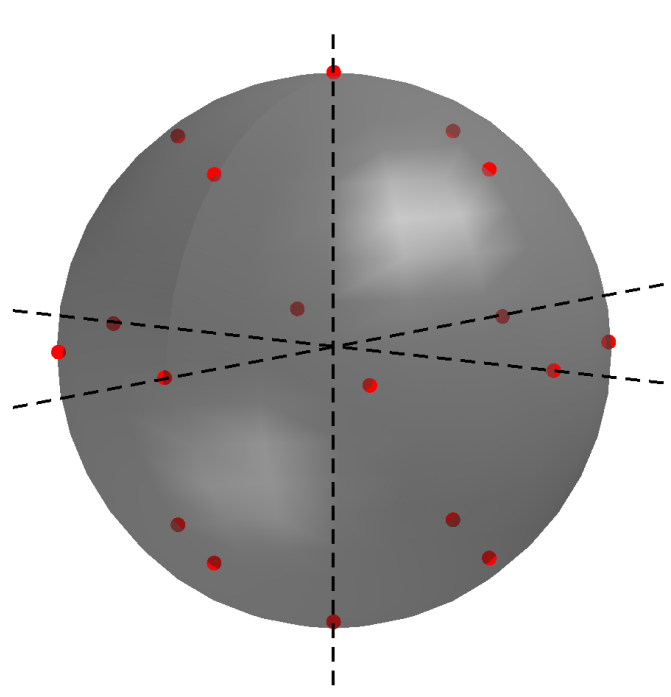

(b) Rendering of the angular grid marks, every 45 degrees in each orthogonal plane, used for positioning the phantom.

Figure 3.2: The phantom used in this study.

Temperature in the scanner room was measured with a digital thermometer in a water bottle, which is kept in the scanner room constantly, and was 21.8 degrees Celcius.

\section{Data Analysis}

Data analysis was performed in FSL [11]. The diffusion tensor, FA, first eigenvalue $\left(\lambda_{1}\right)$ and mean diffusivity (MD) were estimated in native space.

To be able to compare the data from each orientation, each dataset was coregistered to the reference image (tubular phantoms aligned to the $x, y$ and $z$ axes of the scanner). Alignment of the $b 0$ images caused a mis-registration, that is, the registration algorithm finds a perfect registration of the outer edge of the phantom to the reference image, but the internal structures were not aligned. Therefore, we chose to do an edge-detection in the $b 0$ images of each orientation using the edge command, and removed the outer edge by a masking procedure. A mask image was generated with the bet tool in FSL. Next we performed a 12parameter affine registration using FLIRT. The transformation matrix from the registration procedure was then applied using sinc interpolation to the FA, $\lambda_{1}$, $\mathrm{MD}$ and $b 0$ images, including the images from the reference orientation.

We defined 4 regions of interest in the reference image. The ROI voxels were manually chosen and consist of voxels which are completely inside the phantom tubes. One ROI was defined on the tubular phantoms pointing in the $x$ direction 


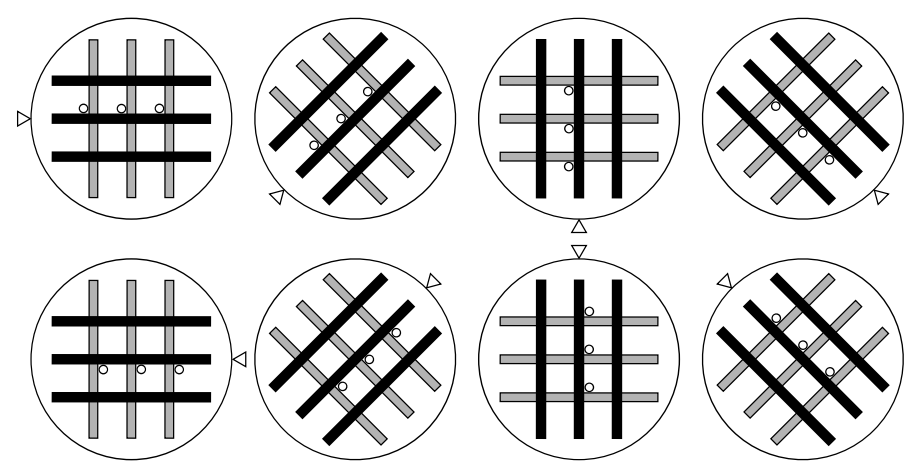

Figure 3.3: Illustration of the rotation procedure around the $x$ axis. The $\triangleright$ is a marker for reference. In the initial position, the $X$ (circle), $Y$ (gray) and Z (black) phantoms are aligned to the $x, y, z$ scanner axes. We define the rotation angle as follows: For the X-phantom, the rotation is along its own long axis, the Y-phantom is rotated $90-135-180-225-270-315-0-45$ degrees and the Z-phantom is rotated o-45-135-180-225-270-315 degrees.

containing 345 voxels, one on the tubular phantoms pointing in the $y$ direction (389 voxels) and one in the $z$ direction ( 383 voxels). Thus, each ROI encompasses voxels from 3 tubular phantoms. A fourth ROI (143 voxels) was defined in a region with water.

The co-registered images were multiplied with each ROI to obtain FA, $\lambda_{1}$, MD and $b 0$ intensity values in each phantom orientation. The results were fed into Matlab R2008b (The Mathworks USA) for further analysis.

To investigate the effect of reorientation and interpolation, a simulation of the reorientation procedure in Matlab was performed. Figure 3.5a shows "phantom" images created with signal intensity 200 [a.u] against o background. Rician noise was added to the image. The image was then rotated in steps of 45 degrees and the data was masked with a ROI encompassing the phantom. The signal intensity deviates around $5 \%$ from the initial intensity when the image is rotated, see figure $3 \cdot 5 \mathrm{~b}$.

\section{$3 \cdot 4$ RESULTS}

In figure 3.6 the $b 0$ images of the phantom in different orientations are shown. Signal intensity inside the phantom pointing up-down in the first orientation compared to the other orientations is changing considerably.

Figure 3.7 shows a rendering of the ROIs in the phantom. The colors represent the orientation of the phantom at the initial position with phantoms aligned to the main $x, y$ and $z$ axes of the scanner. Similar to the rendering in figure 3.7, each color in figures 3.8 and 3.10 represents a set of three phantoms as they were oriented in the initial position. The red color represents the X-phantoms, green 


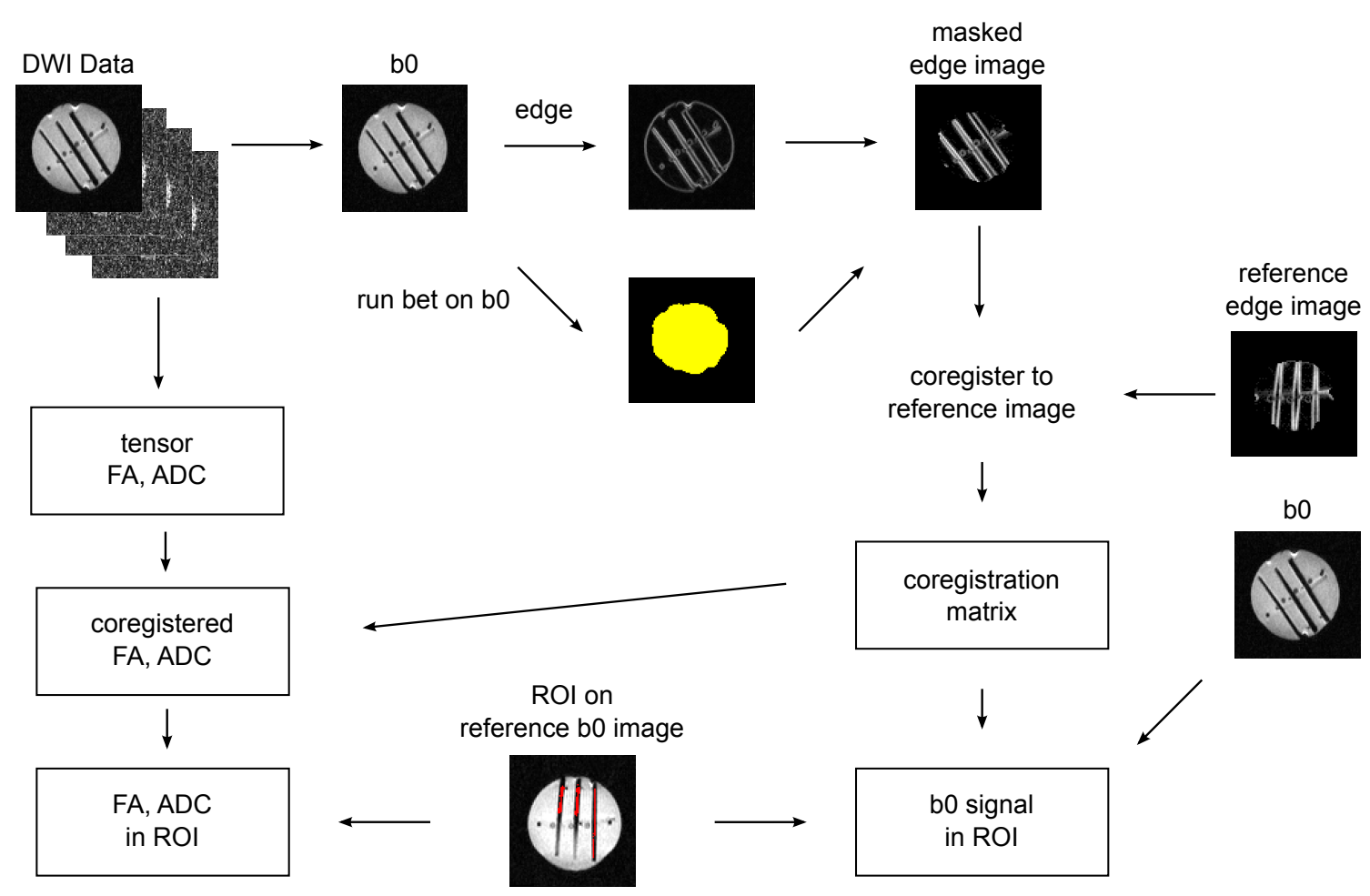

Figure 3.4: Overview of the data analysis procedure.
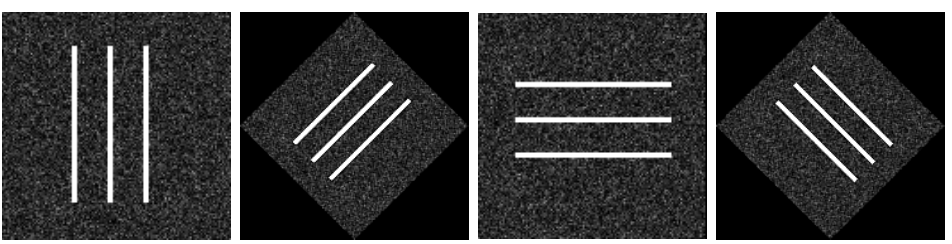

(a) Simulated phantom images. From left to right: o, 45, 90 and 135 degrees rotation.

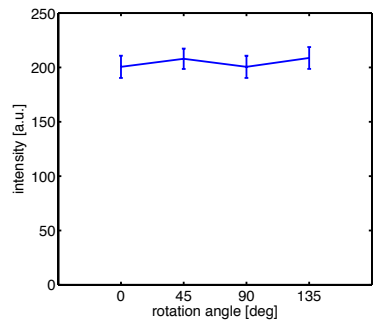

(b) Signal deviation in the simulated phantom as a function of the rotation angle.

Figure 3.5: Simulated effect of interpolation on signal intensity. Signal deviation is around $5 \%$ wrt the original intensity when the image is rotated.
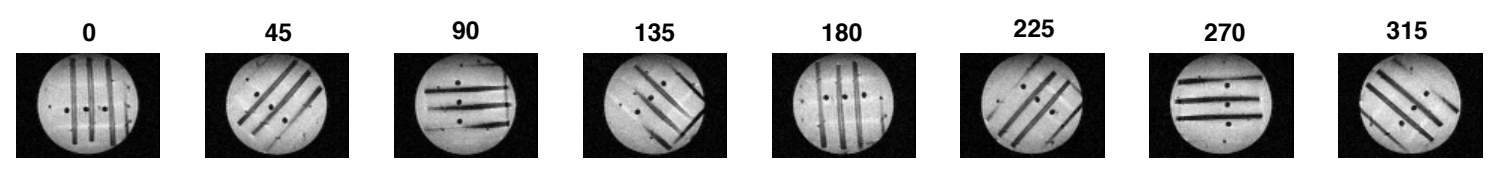

Figure 3.6: $b 0$ images of the phantom at different orientations. 


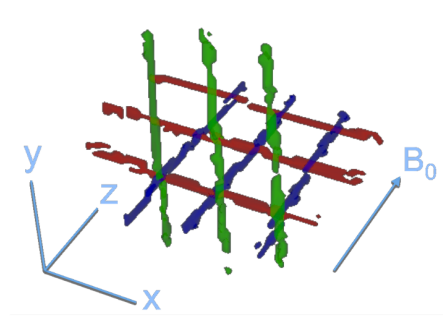

Figure 3.7: Rendering of the 9 tubular phantoms in 3 near-orthogonal planes. Color coding is according to the orientation of the tubes. X-phantom in red, Y-phantom in green and Z-phantom in blue.
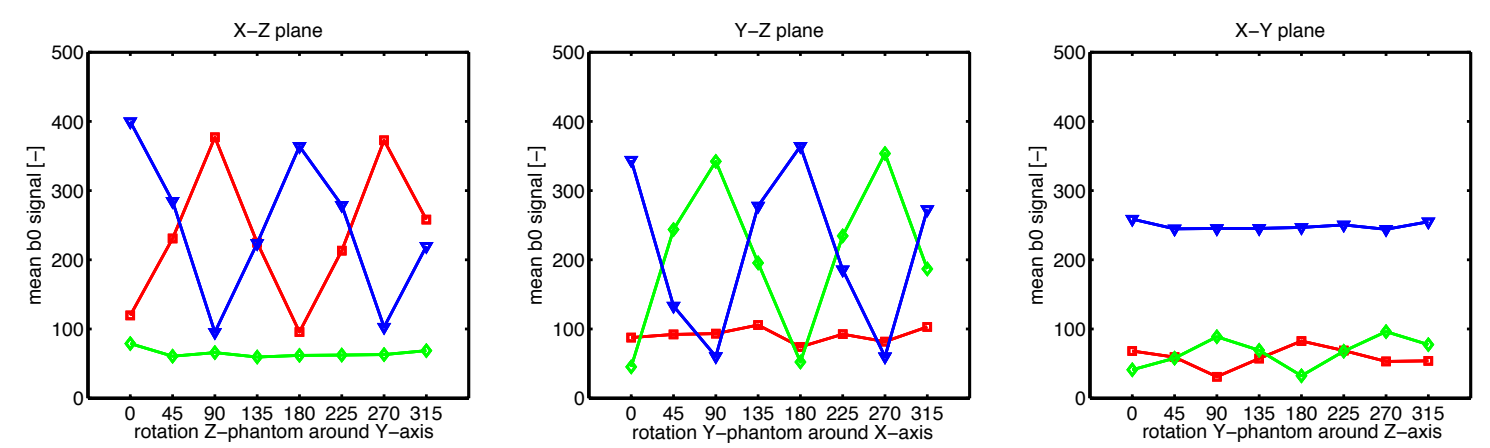

Figure 3.8: $b 0$ signal intensity as function of the phantom orientation. Left: rotation around $y$ axis, middle rotation around $x$ axis and right: rotation around $z$ axis.

Y-phantoms and blue Z-phantoms. From this point on we will refer to 3 tubular phantoms with the same orientation as one phantom.

b0 signal

Striking large variations of $b 0$ signal intensity between different orientations were observed, see figure 3.8. $b 0$ signal intensity is lowest when any of the 3 phantoms is oriented along the $y$ axis, slightly higher when oriented along the $x$ axis and is highest when any of the 3 phantoms is oriented along the $z$ axis. If we look at the initial position (X-phantom $\| x$ axis, Y-phantom $\| y$ axis, Zphantom $\| z$ axis) of the three global rotations, the $b 0$ signal intensity in the $Z$-phantom is largest, followed by the X-phantom and the Y-phantom. The $b 0$ signal intensities in the $X-Z$ plane and the $Y-Z$ plane rotations vary similarly, but the $b 0$ signal in the $X-Y$ plane rotation is considerably lower in $X$-phantom and Y-phantom, while the $b 0$ signal in Z-phantom is lower, but not as substantial.

The mean $b 0$ signal intensity varies in a sinusoidal way in the three experiments. The $b 0$ signal in the phantom that rotates around its own long axis parallel to the global rotation axis shows little variation. Mean $b 0$ signal intensity, see 


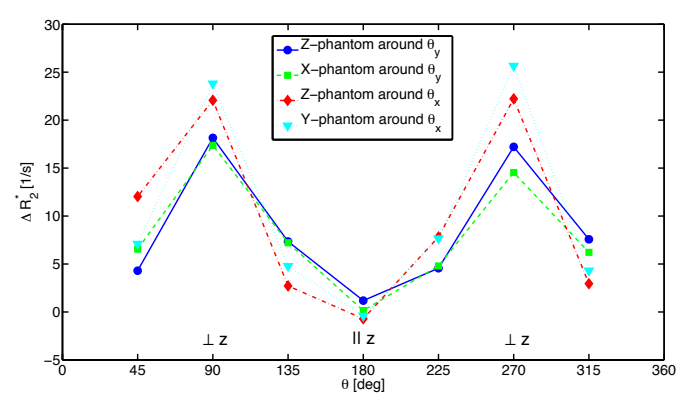

(a) $\Delta R_{2}^{*}$ in the $b 0$ signal as a function of the rotation angle, as calculated by equation 3.7 .

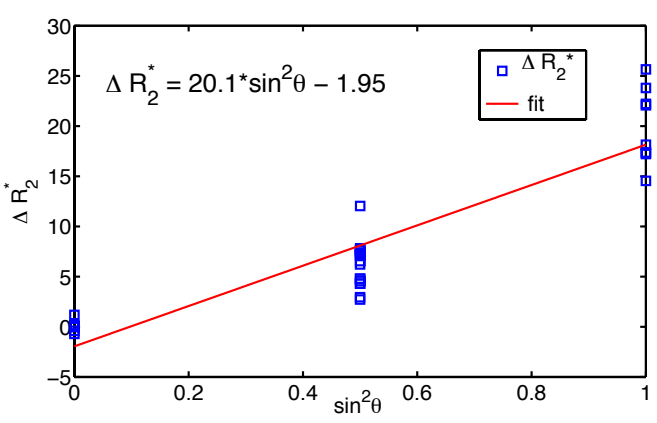

(b) $\Delta R_{2}^{*}$ vs $\sin ^{2}(\theta)$. Squares: calculated values from equation 3.7. Continuous line: linear fit $\Delta R_{2}^{*}=20.1 \cdot \sin ^{2}(\theta)-1.95$.

Figure 3.9: Relation of $\Delta R_{2}^{*}$ and phantom orientation.

table 3.1, of the $X$-phantom in the $X-Z$ plane rotation varies between 95.6-376.9, in the Y-phantom between 59.3-78.8 and in the Z-phantom between 95.3-399.6 [a.u]. Rotated in the $Y-Z$ plane, $b 0$ signal in the $X$-phantom varies between 74.0-105.7, the Y-phantom between 45.1-353.4 and the Z-phantom varies between 59.9-364.0. In the X-Y plane, $b 0$ signal intensities in the X-phantom vary between 31.0-82.5, in the Y-phantom between 32.3-96.1 and in the Z-phantom between 244.0-258.7.

\section{Susceptibility variation}

Susceptibility variations as characterized by $\Delta R_{2}^{*}$ were calculated for the $b 0 \mathrm{im}$ ages with equation 3.7 and shown in figure 3.9. We observe considerable variations in $\Delta R_{2}^{*} . \Delta R_{2}^{*}$ is smallest when the phantom is aligned parallel to the $z$-axis at $\theta=180$ between -0.7-1.2 [1/s]. When the phantom is aligned perpendicular to the $z$-axis, $\Delta R_{2}^{*}$ is largest, between $17.4-23.8$ at $\theta=90$ and at $\theta=270$ between 14.5-25.6. At the positions $\theta=45, \Delta R_{2}^{*}$ varies between 6.6-12.0, at $\theta=135$ between 2.7-7.2, at $\theta=225$ between 4.6-7.8, and $\theta=315$ between 2.9-7.6. The observed rotational dependence of $\Delta R_{2}^{*}$ varies linearly with $\sin ^{2}(\theta)$, and can be characterized by the function $\Delta R_{2}^{*}=20.1 \cdot \sin ^{2}(\theta)-1.95$, see figure $3.9 \mathrm{~b}$.

\section{Diffusion measures}

Figure 3.10 shows diffusion measures $\lambda_{1}, \mathrm{MD}$ and FA. The rotations in the $\mathrm{X}-\mathrm{Z}$ plane (around $y$ axis), $Y-Z$ plane (around $x$ axis) and in the $X-Y$ plane (around $z$ axis) are organised in columns. Mean and standard deviations of $b 0$ signal intensity and FA are shown in table 3.1 and mean and standard deviations of MD and $\lambda_{1}$ in table 3.2 . 

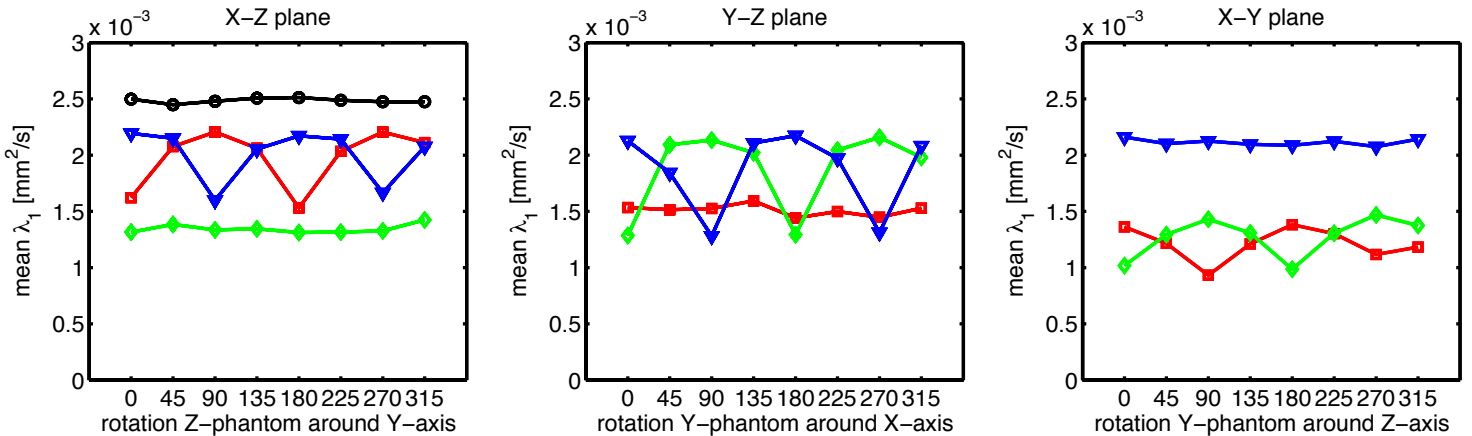

(a) First eigenvalue $\lambda_{1}$ as function of the phantom orientation. Left: rotation around $y$ axis, middle rotation around $x$ axis and right: rotation around $z$ axis. The $\lambda_{1}$ in the ROI containing water is shown in black. As expected, $\lambda_{1}$ of water remains stable wrt phantom orientation.
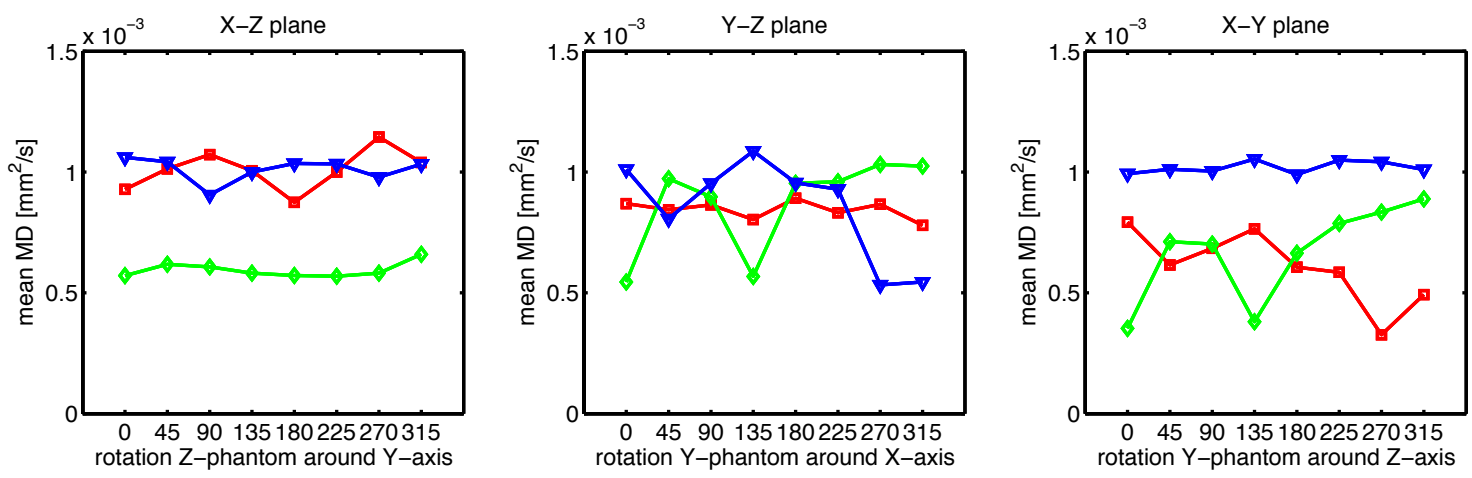

(b) Mean Diffusivity (MD) as function of the phantom orientation. Left: rotation around $y$ axis, middle rotation around $x$ axis and right: rotation around $z$ axis.
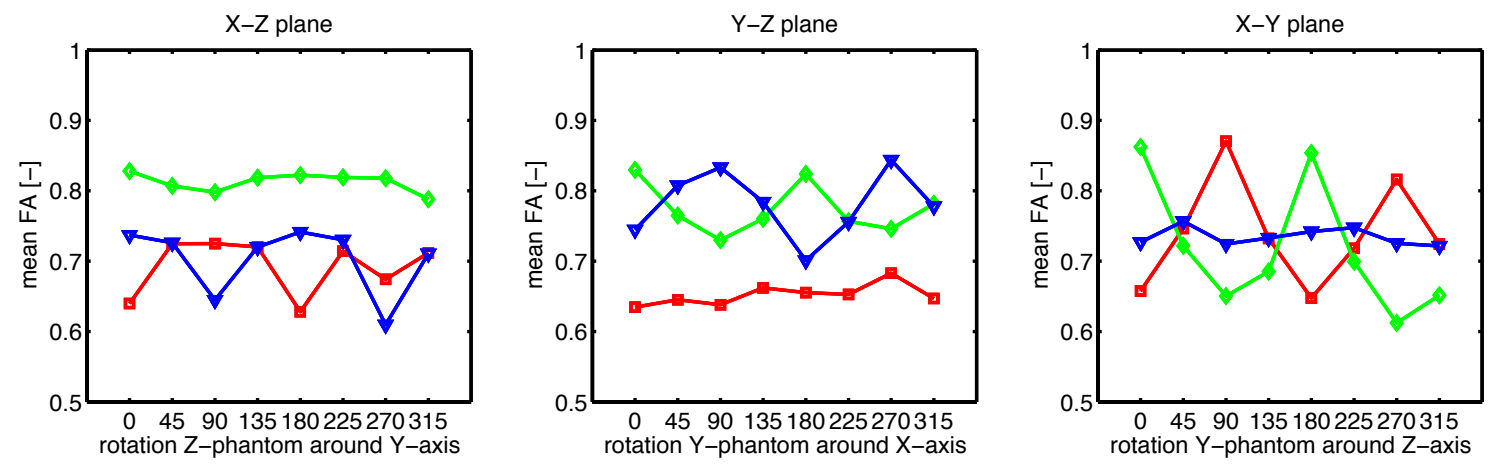

(c) Fractional Anisotropy (FA) as function of the phantom orientation. Left: rotation around $y$ axis, middle rotation around $x$ axis and right: rotation around $z$ axis.

Figure 3.10: The $b 0$ signal and diffusion measures $\lambda_{1}, \mathrm{MD}$ and FA. The rotations in the $X-Z$ plane (around $y$ axis), Y-Z plane (around $x$ axis) and in the $X-Y$ plane (around $z$ axis) are organised in columns. The color coding is equal in each graph and according to initial orientation: red: X-phantom, green Y-phantom and blue Z-phantom. 
Similar behaviour as for the $b 0$ signal is observed for $\lambda_{1} ; \lambda_{1}$ is largest for any of the phantoms aligned parallel to the $z$ axis, and is lower in case any of the phantoms is oriented along the $x$ or $y$ axis.

The highest mean FA is found if any of the phantoms is aligned along the $y$ axis. FA in a phantom aligned to the $z$ axis is lower, and the lowest mean FA values are observed in any of the phantoms aligned to the $x$ axis.

The rotational dependence of the first eigenvalue $\lambda_{1}$ is shown in fig. 3.1oa and table 3.2. $\lambda_{1}$ in the initial position across the 3 experiments is highest in the Zphantom, followed by the X-phantom and is lowest in the Y-phantom. Again, a sinusoidal pattern arises with respect to the rotation angle. $\lambda_{1}$ is similar in the $X-Z$ plane versus the $Y-Z$ plane rotation, and is lower in the $X-Y$ plane rotation for the $X$ and $Y$-phantoms, but similar for the Z-phantom parallel to the $z$ axis in the three experiments.

Mean $\lambda_{1}$ in the $X$-phantom in the $X-Z$ plane rotation varies between $1.531-$ 2.206, in the Y-phantom between $1.312-1.424$ and in the Z-phantom between $1.599-2.194\left[10^{-3} \mathrm{~mm}^{2} / \mathrm{s}\right]$. Rotated in the $\mathrm{Y}-\mathrm{Z}$ plane, $\lambda_{1}$ in the X-phantom varies between $1.441-1.593$, the Y-phantom between $1.284-2.158$ and the Zphantom varies between $1.281-2.172$. In the X-Y plane, $\lambda_{1}$ in the X-phantom varies between $0.932-1.382$, in the Y-phantom between $0.986-1.469$ and in the Z-phantom between $2.075-2.142$.

Mean MD as a function of rotation angle is shown in figure 3.1ob and table 3.2. MD shows a sinusoidal variation when phantoms are rotated in the $X-Z$ plane, but follows a more random pattern in the $Y-Z$ and $X-Y$ planes. The MD shows little variability if a phantom is rotated around its long axis. We observe that MD is highest when a phantom is aligned along the $z$ axis, followed by alignment along the $x$ axis and alignment along the $y$ axis.

Mean MD in the X-phantom in the X-Z plane rotation varies between $0.875-$ 1.145 , in the Y-phantom between $0.569-0.659$ and in the Z-phantom between $0.906-1.061\left[10^{-3} \mathrm{~mm}^{2} / \mathrm{s}\right]$. Rotated in the $\mathrm{Y}-\mathrm{Z}$ plane, MD in the X-phantom varies between $0.779-0.892$, the Y-phantom between $0.545-1.031$ and the Zphantom varies between $0.533-1.086$. In the X-Y plane, MD in the X-phantom varies between $0.327-0.792$, in the Y-phantom between $0.353-0.889$ and in the Z-phantom between $0.990-1.054$.

FA also follows a sinusoidal pattern with respect to rotation angle, as shown in figure 3.10c and table 3.1. In the initial position in the 3 experiments, FA of the Y-phantom is highest, followed by the Z-phantom and X-phantom. We again observe that FA stays at a rather constant level when the phantom is rotated around its own long axis.

Mean FA in the X-phantom in the $X-Z$ plane rotation varies between $0.628-$ 0.725 , in the Y-phantom between $0.798-0.828$ and in the Z-phantom between $0.610-0.742[-]$. Rotated in the $Y-Z$ plane, FA in the $X$-phantom varies between $0.635-0.683$, the Y-phantom between $0.730-0.830$ and the Z-phantom varies 
between $0.701-0.844$. In the $X-Y$ plane, FA in the $X$-phantom varies between $0.648-0.870$, in the Y-phantom between $0.612-0.862$ and in the Z-phantom between $0.724-0.757$.

\section{Reproducibility}

Figure 3.10 demonstrates that the phantoms have a uniform structure and highly similar diffusive properties. The diffusion measures $M \in\left\{F A, b 0, \lambda_{1}\right\}$ have similar values if the phantom has a similar orientation, but with a 'phase' difference. For instance, looking at the X-phantom (red) in figure 3.8, it can be seen that $b 0$ intensity is highly similar in the o versus 180 degrees, 90 vs 270 degrees and 45 , 135, 225 and 315 degrees orientations.

The diffusion measures $M$ are very similar if phantoms swap orientation, that is if the X-phantom is oriented parallel along the $z$-axis, it shows highly similar $b 0$ signal, FA and $\lambda_{1}$ compared to the Z-phantom oriented along the $z$-axis, see figure $3.10(a, b, d)$. This holds for the following orientations:

$$
\begin{aligned}
& \text { for } M \in\left\{F A, b 0, \lambda_{1}\right\}: \\
& M(\mathrm{X} \text {-phantom } \| x \text { axis }) \approx M(\mathrm{Z} \text {-phantom } \| x \text { axis }) \text { rotation in the } \mathrm{X} \text {-Z plane } \\
& M(Z \text {-phantom } \| z \text { axis }) \approx M(X \text {-phantom } \| z \text { axis }) \quad \text { idem. } \\
& M(\mathrm{Y} \text {-phantom } \| y \text { axis }) \approx M(\mathrm{Z} \text {-phantom } \| y \text { axis }) \quad \text { rotation in the } \mathrm{Y} \text {-Z plane } \\
& M(Z \text {-phantom } \| z \text { axis }) \approx M(Y \text {-phantom } \| z \text { axis }) \quad \text { idem. } \\
& M(\mathrm{X} \text {-phantom } \| x \text { axis }) \approx M(\mathrm{Y} \text {-phantom } \| x \text { axis }) \text { rotation in the } \mathrm{X} \text {-Y plane } \\
& M(\text { Y-phantom }|| y \text { axis }) \approx M(X \text {-phantom }|| y \text { axis }) \quad \text { idem. }
\end{aligned}
$$

Mean Diffusivity is very similar if phantoms swap orientation in the $X-Z$ plane, see figure 3.10b. In the other two planes MD follows a more random pattern.

We also observe consistency in the diffusion measures across experimental conditions: The rows in figure 3.10 show the 3 performed rotations and we consistently see that the patterns are similar across experiments, but that values of the diffusion measures change according to orientation of the phantom in the scanner.

\subsection{DISCUSSION}

In this paper we explored the influence of orientation on diffusion measures of an anisotropic diffusion phantom containing tubular phantoms in three nearorthogonal planes. The phantom was rotated in steps of 45 degrees in three planes. We found striking large deviations in the $b 0$ signal intensity, the first 


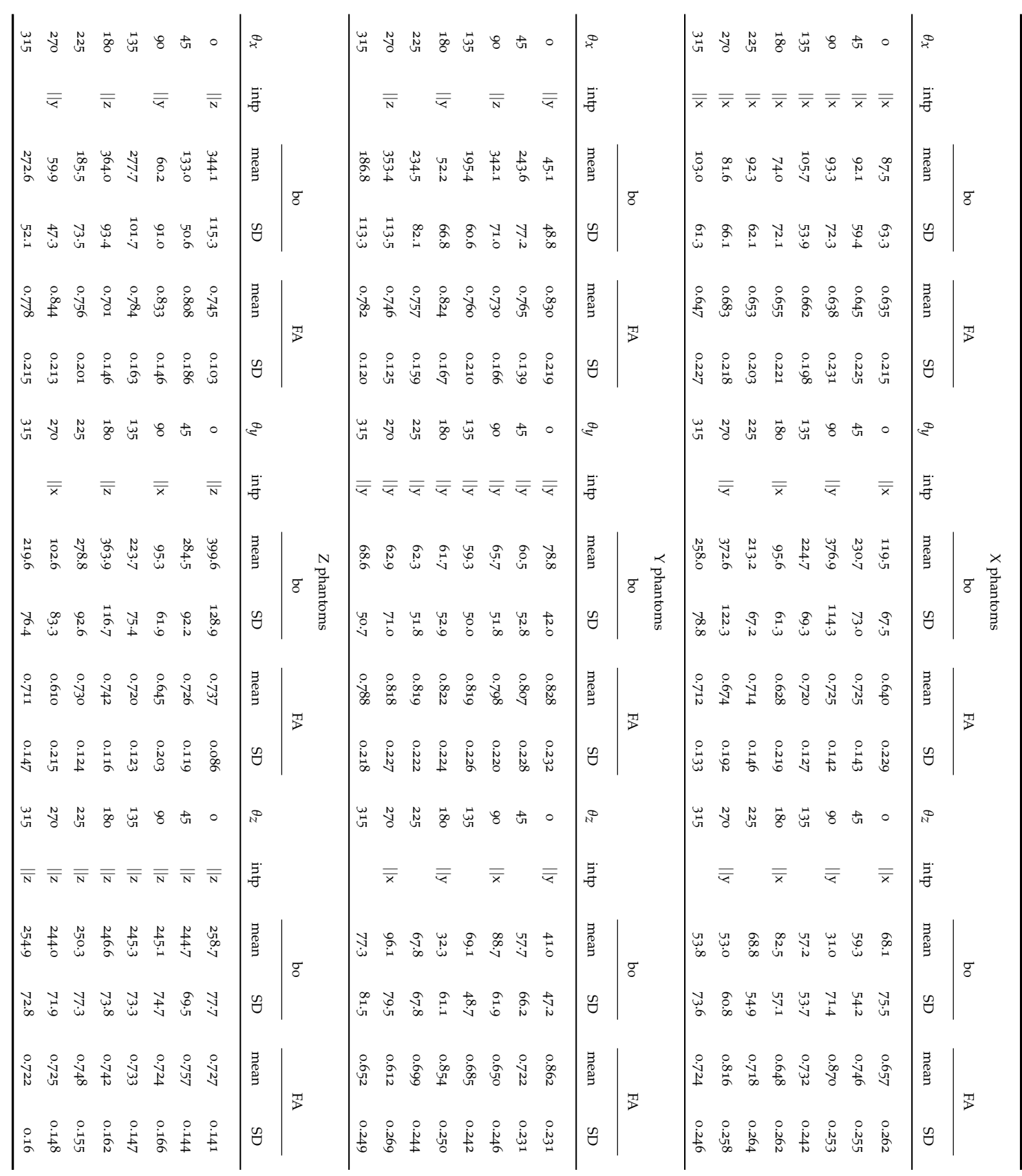




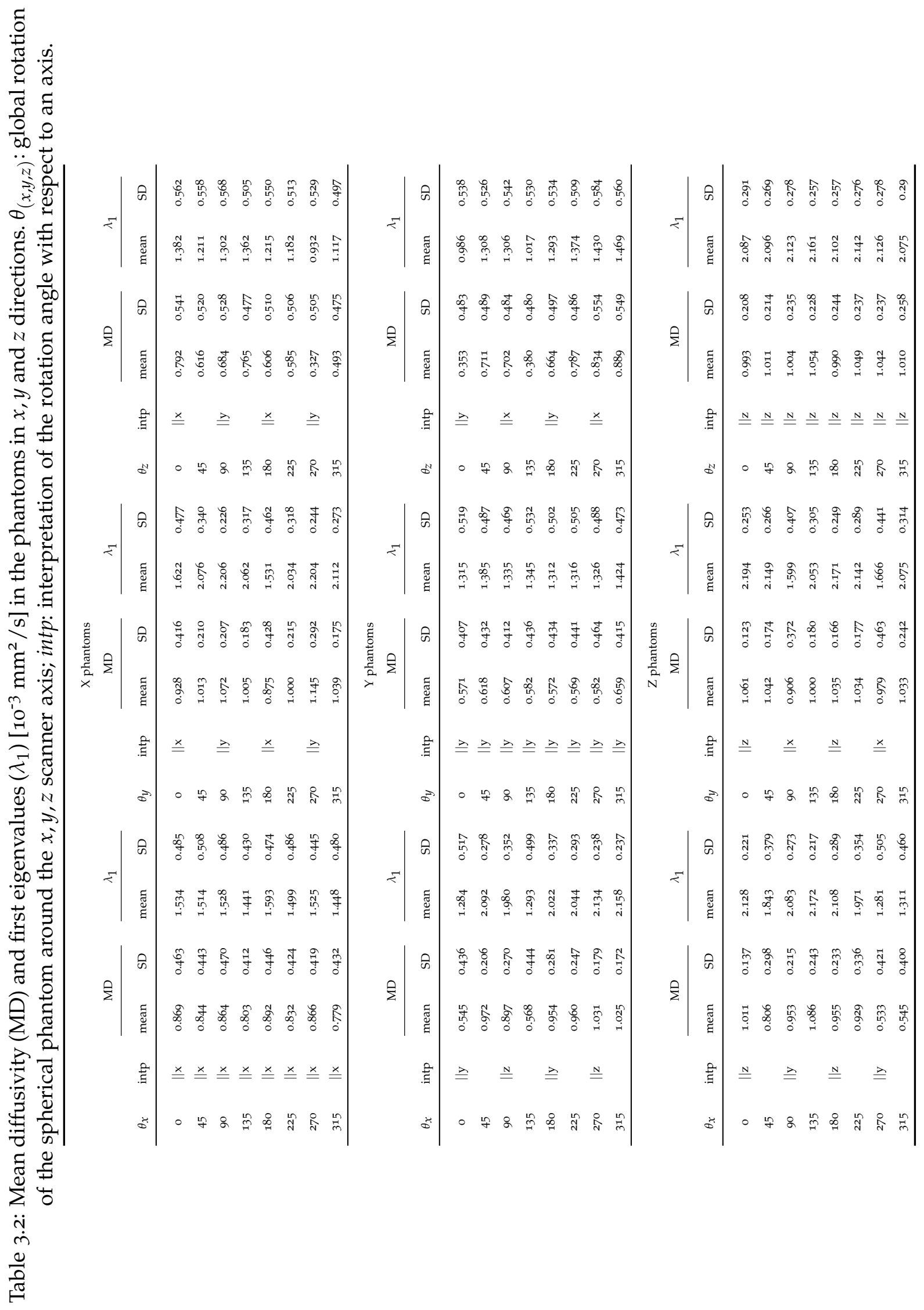


eigenvalue of the diffusion tensor, mean diffusivity and fractional anisotropy with respect to orientation of the phantom in the scanner.

The $b 0$ signal intensity, $\lambda_{1}$ and FA show a sinusoidal variation with respect to orientation in the scanner, while MD shows a more random variation in the $Y-Z$ and $\mathrm{X}-\mathrm{Y}$ plane rotations.

\section{Origin of the variations in diffusion measures: magnetic susceptibility effects}

Given the change in susceptibility when anisotropic white matter tissue is oriented differently in the magnetic field $[12,7,5]$, we hypothesise that the signal variations we observe in the $b 0$ signal (high signal when the phantom is parallel to $B_{0}$ and low when the phantom is perpendicular to $B_{0}$ ) is largely caused by an anisotropic susceptibility effect. We have established the relation $\Delta R_{2}^{*}=20.1 \sin ^{2}(\theta)$.

In brain white matter, a susceptibility change can be induced by deoxygenated blood running through a capillary or vein. The susceptibility change $\Delta R_{2}^{*}$ is around $2.5\left[\mathrm{~s}^{-1}\right]$ which corresponds to a frequency change of approximately $45 \mathrm{~Hz}$ [13] at $3 \mathrm{~T}$. The orientation-dependent susceptibility change in white matter reported in [7] is $\Delta R_{2}^{*}=2.68\left[\mathrm{~s}^{-1}\right]$. The $\Delta R_{2}^{*}$ changes in the phantom are therefore around 4-7 higher than in white matter. Deoxygenated blood induced susceptibility increases when the main magnetic field strength increases [13]. The same is to be expected for orientation-dependent susceptibility changes at higher field strengths.

The origin of local susceptibility changes in WM is not yet clearly understood. Theoretical work attributed the effect to the Lorentzian cavity concept [14] but experimental evidence showed that the main contribution to this effect is the orientation of anisotropic structures relative to $B_{0}$ [12]. In this study, a positive shift in magnetic resonance frequency in excised WM tissue oriented perpendicular to the magnetic field was observed relative to $\mathrm{WM}$ tissue oriented parallel to the main magnetic field. Secondly, in WM perpendicular to $\mathrm{B}_{0}$, susceptibility is higher than in WM tissue oriented parallel to the magnetic field.

The susceptibility effect might be enlarged by gradient-echo effects during the lengthy EPI readout window caused by local magnetic field inhomogeneities $\left(T_{2}^{\prime}\right.$ effects) [15]. The signal decay occurs mainly at the edges of the readout window or the high frequency components in the image [15]. This effect can show large SE-EPI signal changes as was demonstrated experimentally [16]. Parallel imaging can be used to shorten the EPI acquisition tima and could potentially reduce the susceptibility effects.

The influence of susceptibility (an internal gradient) on the signal attenuation in a standard diffusion-weighted pulse gradient echo (PGSE) experiment was demonstrated in [3]. It was shown that, in the presence of an internal gradient, the signal is dominated by slower moving spins and therefore the apparent 
diffusion constant $D_{\text {app }}$ is lower than the real diffusion constant $D$. Large deviations in FA as a result of background gradients were also reported recently [8]. The $b$-value experienced locally in the phantom is therefore likely to be different depending of the orientation of the anisotropic structure relative to the main magnetic field, as indicated by equation 3.11.

The relation between WM fiber orientation, $R_{2}^{*}$ and fractional anisotropy was investigated in [5]. The authors found no specific correlation between $R_{2}^{*}$ and FA. However, in some WM tracts a positive trend between $R_{2}^{*}$ and FA was observed. Accordingly, this might be interpreted as an orientation-dependent variation of FA due to anisotropic susceptibility effects. Our results indicate that a rotationdependent variation in diffusion measures $\mathrm{MD}, \lambda_{1}$, FA exists, but the results obtained from the phantom are likely to be an over-estimation of these measures compared to white matter tissue, since the susceptibility variations are 4-7 times higher than found in white matter.

In order to robustly investigate the observed orientation-dependent change of diffusion measures, numerical simulations should be performed. The PGSE diffusion signal can be simulated by monte-carlo simulations, as in $[17,18,19]$. The influence of additional susceptibility on the spin-echo signal was described in [13]. By combining the two a simulation of the observed effects should be feasible.

\section{Methodological Issues}

The phantom was manipulated by hand in order to rotate it in the scanner. Small additional rotations were unavoidable. We could eliminate additional rotations by fixating the phantom in a frame allowing for one degree of freedom (rotation around one axis).

A preparation phase was performed before each acquisition in a new orientation. Consequently, a new shimming procedure was performed, which could lead to slight variations in the magnetic field inside the phantom between acquisitions. Because the phantom is spherical, shimming effects can be considered small, but it would be interesting to investigate contributions from shimming in the future.

The estimated apparent diffusion constant (ADC) is affected if the scanner's gradient system is not properly calibrated [20]. The offset in ADC is in the order of $0.5-4 \%[20]$, but is scanner dependent. Our system was not calibrated for gradient offsets, which might explain why we see a difference in the $X-Y$ plane diffusive measures with respect to the measures found in the $X-Z$ and $Y-Z$ planes in figure 3.10.

ROI definition was done only in the reference orientation. This ensures an equal amount of voxels in every orientation. If ROI definition is done on each 
orientation separately in native space, the number of voxels in each ROI is very likely to be different in each experiment, thereby introducing a bias in the results.

Partial volume effects may arise when a voxel contains multiple tissue types or materials. When selecting the voxels for the ROIs in the reference image, we carefully selected voxels inside the phantom tubes. However, we cannot be sure that voxels in the other orientations contain only voxels inside the phantom tubes. On the other hand, we only observe only small variations in mean and standard deviations of diffusion measures when phantoms swap positions (i.e. $X$-phantom along $z$ axis versus Z-phantom along $z$ axis). This indicates that there is little variation in ROI voxel position relative to the true spatial position in the rotated phantom tubes.

If the hypothesized relation between anisotropic susceptibility and diffusion measures holds, the found variations in $b 0$ signal, $\lambda_{1}$, MD and FA may be exaggerated if compared to the brain, since the observed susceptibility changes are higher than reported for white matter.

\section{Conclusions}

Using an anisotropic diffusion phantom we have explored a striking effect of orientation of anisotropic structure on diffusion measures such as FA, $\lambda_{1}$ and MD. The measures show a sinusoidal variation with respect to orientation in the scanner. The $b 0$ signal without diffusion weighting is affected in a similar manner and shows large variations when the phantom is rotated. We hypothesise that the observed variations are caused by orientation-dependent susceptibility changes. The results found are likely to be an over-estimation compared to in-vivo white matter measurements, but the phantom provides a highly suitable and easy to manipulate way to investigate these effects.

The observed effects may have large implications on studies employing DWMRI and scalar diffusion measures in multiple human subjects. It shows that very accurate subject positioning is necessary and that, even if structures are aligned to each other, a difference in diffusion measures might be found which is not related to underlying anatomical differences.

\section{REFERENCES}

[1] P Basser and C Pierpaoli. Microstructural and Physiological Features of Tissues Elucidated by Quantitative-Diffusion-Tensor MRI. J. Magn. Reson., 111:209-219, 1996.

[2] DK Jones. The effect of gradient sampling schemes on measures derived from diffusion tensor MRI: a Monte Carlo study. Magn Reson Med, 51:807815,2004 . 
[3] J Zhong, RP Kennan, and JC Gore. Effects of Susceptibility Variations on NMR Measurements of Diffusion. J Magn Reson, 95:267-280, 1991.

[4] DA Yablonskiy, WR Reinus, H Stark, and EM Haacke. Quantitation of T2' Anisotropic Effects on Magnetic Resonance Bone Mineral Density Measurement. Magn Reson Med, 36:214-221, 1996.

[5] A Cherubini, P Péran, GE Hagberg, AE Varsi, G Luccichenti, C Caltagirone, U Sabatini, and G Spalletta. Characterization of white matter fiber bundles with $\mathrm{T}^{*}$ relaxometry and diffusion tensor imaging. Magn Reson Med, 61:1066-72, 2009.

[6] CJ Wiggins, V Gudmundsdottir, D Le Bihan, V Lebon, and M Chaumeil. Orientation Dependence of White Matter $\mathrm{T}^{*}$ Contrast at $7 \mathrm{~T}$ : A Direct Demonstration. Proc ISMRM, 16:237, 2008.

[7] B Bender and U Klose. The in vivo influence of white matter fiber orientation towards $\mathrm{B}(\mathrm{o})$ on $\mathrm{T}^{*}$ in the human brain. NMR in biomedicine, 23:10711076, 2010.

[8] C Rossi, A Boss, P Martirosian, G Steidle, S Capuani, CD Claussen, B Maraviglia, and F Schick. Influence of steady background gradients on the accuracy of molecular diffusion anisotropy measurements. Magn reson imaging, 26:1250-8, 2008.

[9] Anisotropic hardware phantom for DW-MRI applications. http://www. brainvoyager.com/diffusionphantoms. Brain Innovation B.V., Maastricht, NL.

[10] P Pullens, A Roebroeck, and R Goebel. Ground Truth Hardware Phantoms for Validation of Diffusion-Weighted MRI Applications. J Magn Reson Imag, 488:482-488, 2010.

[11] SM Smith et al. Advances in Functional and Structural MR Image Analysis and Implementation as FSL. NeuroImage, 23(S1):208-219, 2004.

[12] J Lee, K Shmueli, M Fukunaga, P Van Gelderen, H Merkle, AC Silva, and JH Duyn. Sensitivity of MRI resonance frequency to the orientation of brain tissue microstructure. PNAS, 107:5130-5, 2010.

[13] K Uludag, B Müller-Bierl, and K Ugurbil. An integrative model for neuronal activity-induced signal changes for gradient and spin echo functional imaging. NeuroImage, 48(1):150-65, 2009.

[14] X He and DA Yablonskiy. Biophysical mechanisms of phase contrast in gradient echo MRI. PNAS, 106:13558-13536, 2009. 
[15] R Birn and PA Bandettini. The Effect of T2' Changes on Spin-Echo EPIderived Brain Activation Maps. Proc ISMRM, 10, 2002.

[16] RM Birn, JA Bodurka, N Petridou, and PA Bandettini. Experimental determination of the effect of T2' changes in spin-echo EPI. Proc ISMRM, 11:997, 2004 .

[17] MG Hall and DC Alexander. Convergence and Parameter Choice for MonteCarlo Simulations of Diffusion MRI. IEEE Trans. Med. Imag., 28:1354-1364, 2009 .

[18] FB Laun, S Huff, and B Stieltjes. On the effects of dephasing due to local gradients in diffusion tensor imaging experiments: relevance for diffusion tensor imaging fiber phantoms. Magnetic resonance imaging, 27:541-8, 2009.

[19] E Fieremans, Y De Deene, S Delputte, MS Ozdemir, Y D'Asseler, J Vlassenbroeck, K Deblaere, E Achten, and I Lemahieu. Simulation and experimental verification of the diffusion in an anisotropic fiber phantom. Journal of Magnetic Resonance, 190:189-99, 2008.

[20] Z Nagy, N Weiskopf, DC Alexander, and R Deichmann. A method for improving the performance of gradient systems for diffusion-weighted MRI. Magn Reson Med, 58:763-768, 2007. 
this Chapter is BASed on Pim Pullens, Joost Mulders, Alard Roebroek and Rainer Goebel. An iterative method to correct for motion and eddy currents in DW-MRI data. In preparation 


\section{ABSTRACT}

Gradient-echo echo planar imaging (GE-EPI) commonly used for diffusion weighted magnetic resonance imaging (DW-MRI) can suffer from i) eddy-current (EC) induced distortions and ii) bulk-motion artefacts.

We present an iterative method to correct for both slice-specific affine EC distortions and volume bulk-motion in DW-MRI data. Our method avoids the problem of overcorrection in volume-based affine registration methods, as well as the problem of bulk motion for slice-based EC-correction methods, and does not rely on phantom calibration. The method iterates a two-step procedure of intensity-gradient based motion correction and a cross correlation method for EC correction. Validation using an in-vivo high-angular resolution DWI data set shows superior performance of the proposed method over existing methods for affine image registration and EC distortion correction. We discuss interpolation and iteration convergence for the method.

The improved alignment and correction of DW-MRI images could benefit many applications such as comparative white matter micro-structure investigations, fiber tractography and clinical diagnostics.

\subsection{INTRODUCTION}

Diffusion-weighted magnetic resonance imaging (DW-MRI) has found many applications in recent years, such as comparative white matter microstructure investigations, fiber tractography and clinical diagnostics. In DW-MRI the crucial contrast is that between images without diffusion weighting (the "bo image") and images with diffusion weighting ("DW images"). Therefore, all calculation results depend upon an optimal registration of the DW images to the bo images. Pulsed gradient spin echo (PGSE) diffusion weighting with a gradient-echo echo planar imaging (GE-EPI) readout is by far the most used acquisition method for DW-MRI. In DW-EPI in particular, the problems of i) eddy-current (EC) induced distortions and ii) bulk-motion artefacts are prominent.

Diffusion contrast is achieved by applying large and fast-changing gradient pulses (DW gradients) with a directional encoding. Eddy currents in DW-MRI are formed in conductive parts of the MR scanner induced by the strong DW gradients. Consequently, the eddy currents themselves create additional magnetic gradients in the system, which are not accounted for in the image reconstruction and create artefacts in the data [1]. Thus, eddy current induced distortions arise from an interaction of the image acquisition gradients (which are slice specific) and the DW gradients applied. The effect of eddy currents can be reduced during the acquisition by using adapted pulse sequences such as a twice-refocussed spin-echo sequence [2], a dual spin-echo sequence [3] or adapting the gradient waveform [3]. However, EC distortions can never be fully eliminated, necessitat- 
ing post-processing of the DW-MRI data to remove distortions. As DW-MRI experiments typically take 10-45 minutes, subject motion is unavoidable (especially in a clinical setting where patients may not be compliant to the MRI procedure) and needs to be corrected for as well.

There are currently two main approaches to correcting EC distortions. First, a $3_{\mathrm{D}}^{\mathrm{D}}$ volume based affine registration of the DW images to the bo images [4]. This method has been shown to sometimes over-correct the distortions in the readout direction [5]. Second, a brute-force slice-based cross correlation approach (ICC) [6]. This slice-based method takes into account that DW distortions are, in principle, slice specific and are only present in the phase encoding direction. The method uses phantom-based calibration to estimate the distortions, which requires regular re-calibration when the scanner is updated or when a new sequence is used. A second issue with this method is that the performance of this method is greatly affected by bulk motion and does not perform well at higher b-values $\left(>300 \mathrm{~s} / \mathrm{mm}^{2}\right)$, because of the large contrast differences between low and high $b$-value images. If we use the contribution of each gradient direction to correct the distortions, this method has been demonstrated to perform well at higher b-values [3].

We present an iterative method to correct for both slice-specific affine EC distortions and volume bulk-motion in DW-MRI data. Our method avoids the problem of overcorrection in volume-based affine registration methods, as well as the problem of bulk motion for slice-based EC-correction methods, and does not rely on any type of calibration.

\subsection{METHODS}

We propose a procedure which calculates motion parameters and eddy current distortions independently on the acquired data set. The resulting parameters are combined in a final transformation matrix and therefore require only a single interpolation step. The procedure may be run iteratively if necessary.

The motion correction step (Normalized Gradient Field Motion Correction) operates on a 3-D data volume, while the eddy current correction (Iterative CrossCorrelation using diffusion gradient information) operates on a slice-by-slice level.

\section{Normalized gradient field motion correction algorithm}

The motion correction algorithm is a volume-based method, minimizing a distance measure. The 'Normalized Gradient Field' distance measure is proposed by Haber and Modersitzki [7] as an alternative to Mutual Information. It is based on the notion that two images can be considered similar, if intensity changes occur at the same locations. Encoding intensity changes by normalized image gra- 
dients minimizes the effect of the absolute image magnitude. Since the gradient direction gives purely geometric information, this distance measure is very suitable for the registration of images with different contrast such as the registration of DW images to bo images.

Since the gradient fields are normalized, the norm of the vector product (crossproduct) of the two gradient vectors is only related to the sine of the angle between the vectors: $|a \times b|=|a||b| \sin \varphi$. For two related points, $p$ in reference image $R$ and $q$ in transforming image $T$, we look at the vectors $\mathbf{n}(R, p)$ and $\mathbf{n}(T, q)$. To register the images, the square of this cross product needs to be minimized:

$$
D(T, R)=\|\mathbf{n}(R, x) \times \mathbf{n}(T, y)\|^{2} .
$$

To avoid problems with areas where the image is constant and highly sensitive to small intensity changes like noise, the following regularized normalized gradient field is defined.

$$
\begin{array}{ll}
\mathbf{n}_{\varepsilon(I, x)} & :=\frac{\Delta I(x)}{\|\Delta I(x)\|_{\varepsilon}} \\
\|\Delta I(x)\|_{\varepsilon} & :=\sqrt{\Delta I(x)^{T} \Delta I(x)+\varepsilon^{2}},
\end{array}
$$

where $\Delta I(x)$ is the local intensity change.

The choice of parameter $\varepsilon$ determines what what is considered as a significant gradient and what is considered as insignificant variations. Here, volume based $(3 \mathrm{D})$ rigid body registration (translations and rotations) was used, which means the registration only corrects for the subject motion and not for the EC distortions. A Gauss-Newton optimization method was used to iteratively find the optimal rotation and translation parameters. The image gradients $\Delta I$ were calculated using Gaussian derivatives at the scales $(\sigma)$ of 4,3 and 2.4 millimeter [8].

\section{Eddy current correction by cross correlation using diffusion gradient information (gICC)}

Assuming a Cartesian $k$-space encoding of a slice we define the phase-encoding direction as the $y$ direction, the readout direction as $x$ and the slice normal as $z$. Shear in the slice is a result of excess magnetic gradients induced by eddy currents in the $x$-direction; scaling is the result of excess gradients in the $y$ direction; and translation is the result of excess gradients in the $z$ direction.

Eddy currents distort the image in the phase-encoding direction, and result in a magnification, a shear or a translation in the image. The ICC algorithm estimates the magnification, shear and translation $(M, S, T)$ in a phase encoding column of the data by cross-correlating columns of the distorted data with corresponding columns in a reference image. The algorithm as described in [9], operates as follows: 
1. Take each column $C d$ of the distorted image and scaling it around its center point with a scaling factor $m \in[0.85,1.15]$, with steps of 0.005 , to obtain $\mathrm{Cdm}$. The normalized cross-correlation series $\mathrm{CCm}$ between a column $\mathrm{Cu}$ from the reference image, which is the bo image in this algorithm, and $\mathrm{Cdm}$ is then calculated containing all relative shifts. Interpolation of both columns prior to cross-correlation computation allows detection of subpixel shifts.

2. The maximum correlation across all scales $m$ leads to the optimal scaling factor $M^{\prime}$. The shift of the maximum in $\mathrm{CCm}$ is taken to be the optimal translation $T^{\prime}$ for that column.

3. $M^{\prime}$ and $T^{\prime}$ are calculated for all phase-encoding columns in the image. A linear regression of all $T^{\prime}$ s over all columns is performed. The constant of the regression is the translation $T$ and the slope of the regression line is the shear $S$. The final scale $M$ is found by averaging the scales across all phase encoding lines.

The major drawback of the ICC method is that it only performs well in images with low $b$-values. In higher $b$ value images, the contrast in the reference $b 0$ image and the contrast in the diffusion-weighted image differ too much for the method to work properly. To address this issue, the gradient directions and strength can be used to model the distortion parameters [3].

The total distortion in an image is equal to the linear sum of the three distortions induced by the $x, y$ and $z$ components of the diffusion gradients [3]. In a given slice, the gICC algorithm first finds the translation $D t$, scale $D m$ and shear $D s$ for each gradient direction using the original ICC algorithm [6] as described before. However, the reference image is now a DW-image, having a similar contrast to the DW-images that need correction. The parameters for this slice are stored in vectors $\mathbf{D}_{t}, \mathbf{D}_{s}$ and $\mathbf{D}_{m}$ respectively.

The distortion parameters are then adjusted for the influence of each diffusion gradient $g_{i}$. The first step is to construct three intermediate matrices T, $\mathbf{S}, \mathbf{M}$ which contain 'global' information of all translations, shears and magnifications, see eqs 4.3-4.5. Then, the gradient-adjusted distortions $D t_{g}, D m_{g}$ and $D s_{g}$ for all gradients (including the reference) are calculated using eqs 4.6-4.8.

$$
\begin{aligned}
\mathbf{T} & =\left(\mathbf{G}^{\prime T} \cdot \mathbf{G}^{\prime}\right)^{-1} \cdot \mathbf{G}^{\prime T} \cdot \mathbf{D}_{t} \\
\mathbf{S} & =\left(\mathbf{G}^{\prime T} \cdot \mathbf{G}^{\prime}\right)^{-1} \cdot \mathbf{G}^{\prime T} \cdot \mathbf{D}_{s} \\
\mathbf{M} & =\left(\mathbf{G}^{\prime \prime T} \cdot \mathbf{G}^{\prime \prime}\right)^{-1} \cdot \mathbf{G}^{\prime \prime T} \cdot \mathbf{D}_{m} \\
D t_{g i} & =\mathbf{G}_{i} \cdot \mathbf{T} \\
D s_{g i} & =\mathbf{G}_{i} \cdot \mathbf{S} \\
D m_{g i}-1 & =\mathbf{G}_{i} \cdot \mathbf{M},
\end{aligned}
$$


where the rows of matrices $\mathbf{G}^{\prime}$ and $\mathrm{G}^{\prime \prime}$ are formed by

$$
\begin{aligned}
\mathbf{G}^{\prime} & =\left[G_{i x}-G_{\mathrm{ref}_{x}} G_{i y}-G_{\mathrm{ref}_{y}} G_{i z}-G_{\mathrm{ref}_{z}}\right] \\
\mathbf{G}^{\prime \prime} & =\left[G_{i x}-D m_{i} G_{\mathrm{ref}_{x}} G_{i y}-D m_{i} G_{\mathrm{ref}_{y}} G_{i z}-D m_{i} G_{\mathrm{ref}_{z}}\right],
\end{aligned}
$$

$i \neq$ the reference gradient "ref" and where $G_{i j}$ is the gradient direction $\mathbf{g}$ in units of $\mathrm{mT} / \mathrm{m}$.

\section{Implementation and adaptations}

The gICC and the motion correction method were implemented in Matlab and as a plug-in for BrainVoyagerQX 2.2 (Brain Innovation BV, Maastricht NL) in $\mathrm{C} / \mathrm{C}++$.

We adapted the motion correction algorithm such that the user is given the choice to use only the bo volumes (if these are regularly inter-dispersed between the diffusion weighted volumes) for estimation of motion correction parameters, or that the complete data set (bo + diffusion weighted images) is used. The motion correction parameters are used to correct the complete data set. When motion is only detected on the bo volumes, linear interpolation is used to find the motion correction parameters belonging to the diffusion weighted images.

In the original gICC algorithm by Zhuang et al [3], the first non-zero gradient was set as the reference gradient. This might not always be the best choice, since the distortions induced by the first non-zero gradient are arbitrary. We assign as reference the image that is affected in an equal amount by eddy currents in the $x, y$ and $z$ direction, where we assume for the moment that the amount of distortion caused by the $x, y$ and $z$ gradients is equal. In practice, we search for the gradient direction which is closest to a vector pointing at 45 degrees from each axis $\mathbf{v}_{r e f}=\left[\frac{1}{3} \sqrt{3} \frac{1}{3} \sqrt{3} \frac{1}{3} \sqrt{3}\right]^{T}$ by finding the gradient direction with a minimal inner product with $\mathbf{v}_{r e f}$, that is $\min \left(\mathbf{v}_{r e f} \cdot \mathbf{g}_{i}\right)$, and taking the corresponding volume as the reference image.

To speed up the calculations we calculated the distortions $D t, D m$, Ds only in the most severely affected volumes, that is the volumes with a gradient direction close to $[ \pm 1,0,0],[0, \pm 1,0]$ or $[0,0, \pm 1]$. The distortions for the rest of the volumes follow from the gradient adjustment step.

We have also found that the gICC algorithm is highly susceptible to background noise. We therefore estimate the background noise by calculating the signal intensity in non-brain voxels (mean signal intensity in rows 2 to 7 of the image) and apply this threshold to the data before calculating the normalized cross-correlation between $C d$ and $C u$ and use only those columns with enough relevant data points (brain voxels).

The parameters estimated from the motion correction (whole volume rotation and translation) and eddy current distortion calculation (slice by slice translation, 
scaling and shear) were combined into one transformation matrix. We use Bspline interpolation to correct the data.

The rotation information from the motion correction step was used to rotate the gradient directions to the new orientation of the data as is required, that is $\mathbf{g}_{i}^{\prime}=\mathbf{R}_{i} \cdot \mathbf{g}_{i} \cdot\left(\mathbf{R}_{i} \cdot \mathbf{g}_{i}\right)^{T}$, with $\mathbf{g}_{i}^{\prime}$ the reoriented gradient for diffusion direction $i[10]$.

The iterative algorithm describing the correction method is displayed in Procedure 1 . The \% variance of the first principal component is used as a convergence criterion. Since a better motion correction will be possible after each EC correction, the scale at which the Normalized Gradient Field registration operates in the motion correction is chosen to become more detailed after each iteration.

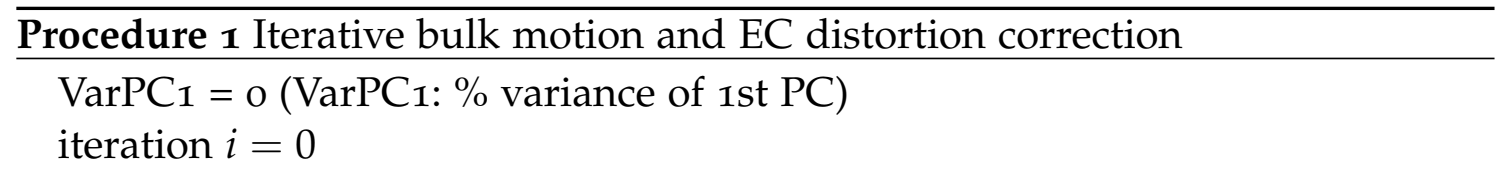

Require: Select to use complete data set or bo volumes only for motion correction

for iteration $i$ do

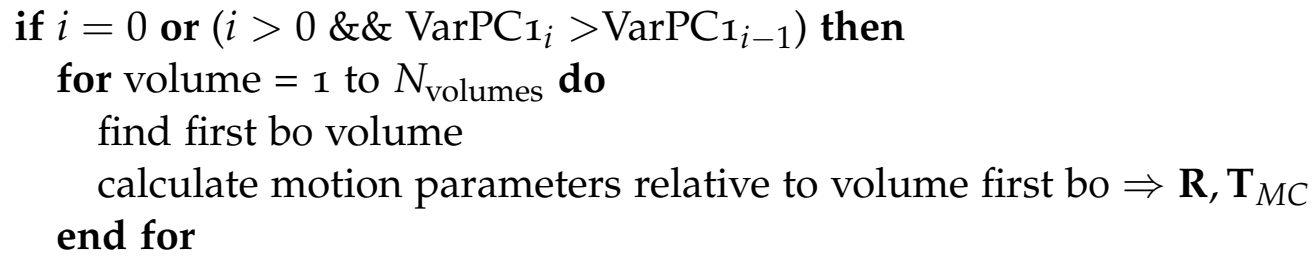




\section{Performance measures}

PRINCIPAL COMPONENT ANALYSIS To test the performance of our method, we use principal component analysis (PCA), which has been demonstrated to be a powerful tool to check co-registration accuracy in DW-MRI data $[5,11]$. PCA transforms the original data set into a new set of uncorrelated images. The first component (image) is the one explaining most of the variance in the data, the second component the second largest variance and so forth. PCA can show interesting features which are not directly visible in the original data set [11].

PCA as implemented in Matlab R2008b (The Mathworks, USA) was performed on a $N^{2} \times P$ matrix of the diffusion-weighted data (excluding the bo images), where the columns are formed by the $N \times N$ voxels in an image and $P$ is the number of diffusion-weighted volumes. To normalize, each image is divided by its standard deviation [5]. The performance measure (percentage of variance in each component $[i=1, \ldots, P])$ is calculated from the eigenvalues of the covariance matrix $\left[\lambda_{1}, \ldots, \lambda_{P}\right]$

$$
\% \text { Variance }=100 \cdot \frac{\lambda_{i}}{\sum_{i=1}^{P} \lambda_{i}} .
$$

STANDARD DEVIATION ACROSS IMAGES The standard deviation across the volumes of the data is a powerful way to visualize possible misalignments between images [12]. Any misalignment in the diffusion-weighted volumes will show up as a bright rim or spot in the standard deviation image $\left(S_{D W I}\right.$-image $)$ of these volumes. The standard deviation across the $b 0$ volumes ( $S_{b 0}$ image) provides an efficient way to detect pulsation artefacts [12]. This measure can only be used qualitatively, since the standard deviation across the images has no physical value.

\section{Data acquisition}

A high-angular resolution DW-MRI data set was acquired on a healthy subject: male 26 years old on a $3 \mathrm{~T}$ Siemens Allegra system with Gmax $=40 \mathrm{mT} / \mathrm{m}$. 80 slices, 109 diffusion directions and 10 interleaved bos were acquired using a twice refocussed spin-echo echo-planar imaging sequence; $b=1000 \mathrm{~s} / \mathrm{mm}^{2}$. FOV $256 \times 256,128 \times 128$ matrix, resulting in $2 \mathrm{~mm}^{3}$ iso-voxels. TR/TE 980o/82 ms, making up for a total scanning time of 19 mins $26 \mathrm{~s}$.

\section{Corrections}

The data set was corrected for motion and distortions. We investigate five options: 
МС-во motion correction using only the bo volumes

MC-CM motion correction using the complete data set

MC-ECC-во motion correction using the bo volumes and eddy current correction using the complete data set

MC-ECC-CM motion and eddy current correction using the complete data set.

FSL as a reference the data was processed in FMRIB's Diffusion Toolbox in FSL 4.1.5 [4] with the eddy_correct command. This procedure corrects for the distortions, and for simple head motion, using affine registration to a reference volume [4]. We used the second volume (the first bo) as the refence volume. Note that motion and eddy current correction are processed simultaneously on a volume-by-volume basis.

Secondly, we corrected the data set using the diffusion weighted volumes for motion and eddy currents with multiple iterations of the algorithm. The most extensive correction procedure MC-ECC-CM takes approximately 10 minutes on a MacBook Pro $2.8 \mathrm{GHz}$ Intel Core 2 Duo with 4 Gb of RAM, using a single core.

\section{$4 \cdot 3$ RESULTS}

Amount of motion and eddy current distortion

In figure 4.1 the amount of motion and distortions due to eddy currents are shown. The amount of motion is small in this data set, with displacements smaller than $1 \mathrm{~mm}$ and rotation below 2 degrees. The motion parameters show a step-wise behaviour, with a plateau between each $b 0$ volume. The contrast difference between successive DWI images is probably too low, therefore it is hard for the NGF method to detect motion between successive DWI volumes.

\subsubsection{Principal component analysis}

SINGLE ITERATION CORRECTION FOR MOTION AND EDDY CURRENTS PCA of the uncorrected and corrected data sets reveals properties of the data which are not directly visible. In the original uncorrected data (figure 4.2a), a misregistration error appears in PC 7 and 8 as a white rim, indicated by an arrow. The motion corrected data using only the bo images in figure $4.2 \mathrm{~b}$ shows a reduction of this misregistration error in PC's 7 and 8, and a further improvement is shown in figure $4.2 \mathrm{C}$, where we used the complete data set for motion correction. There is no white rim visible, so the misregistration error in $\mathrm{PC}_{7}$ has almost completely disappeared and in PC 8 it was completely removed by the correction. 

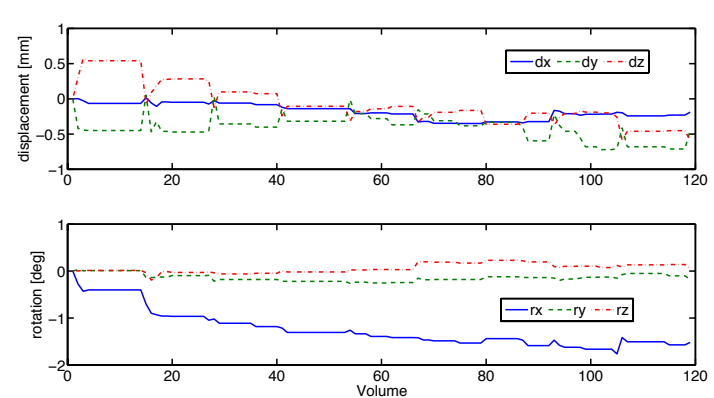

(a) Motion correction parameters found using the complete dataset.

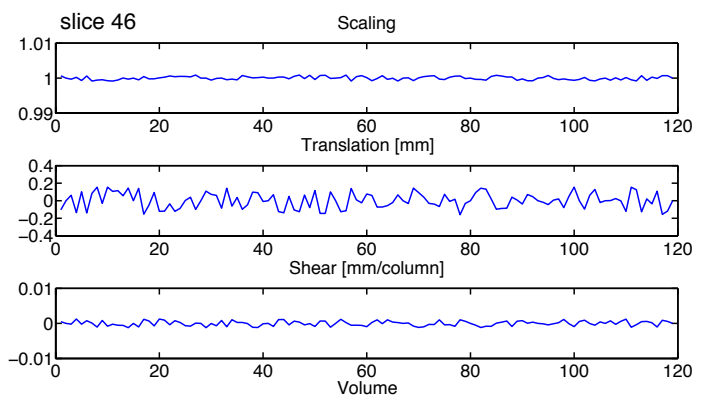

(b) Eddy Current distortions for slice 46.

Figure 4.1: Motion and distortion parameters calculated with our method using the complete data set.

In figure $4.3 \mathrm{a}$ the quantitative reduction in variance is shown. The variance of the first PC is highest $(77.79 \%)$ compared to the uncorrected data set $(73.93 \%)$, when the complete data set is used for motion correction. When only the bo volumes are used for motion correction, the first PC variance is $76.98 \%$.

When we look at the higher components, a clear reduction in PC variance is seen in components 7 and higher with respect to the uncorrected data. There is only a small reduction in variance when the data is corrected for eddy currents, but in figure $4.3 \mathrm{~b}$ it is shown that the mis-registration error is reduced in the lower part of the image and therefore, eddy current correction will still be necessary for optimal image registration.

MULTiPle iterations of THE Algorithm Secondly, we corrected the data set using the diffusion weighted volumes for motion and eddy currents with multiple iterations of the algorithm until the second and higher PCs did not change significantly. Figure 4.6 shows that if two iterations of the algorithm are used, the percent variance of the first PC is higher than when it is ran once (83.15\% versus $77.79 \%)$. The 2nd and higher PCs are lower when the algorithm is ran twice than when one iteration is used. Running the algorithm for a third iteration does not change the percentage of variance of the components significantly.

FSL CORRECTION When the data set is processed in FSL, the percent variance in the first component is $90.10 \%$, and the percent variance in the 2 nd and higher components is slightly lower than the percent variance after two iterations of our algorithm. At first sight, FSL's method seems to perform better, but if we take a closer look at the individual components, we see an overestimation of the distortions in PC 7 of the data in figure $4.6 \mathrm{~b}$. 

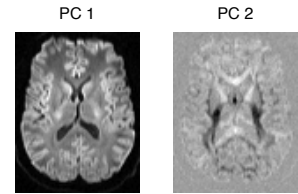

PC 5

PC 6

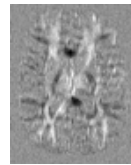

PC 9
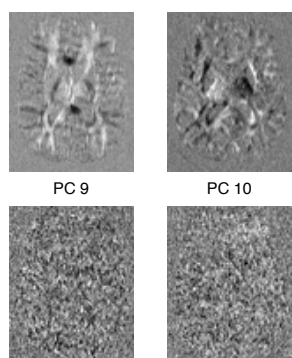

PC 10

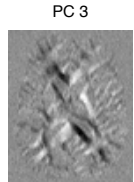

PC 7

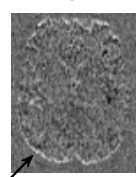

PC 11
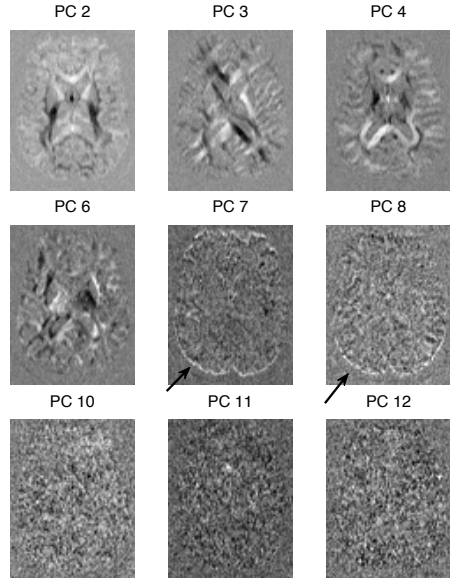

PC 8

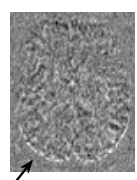

PC 12

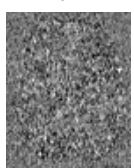

(a) PCA decomposition of the original data set.
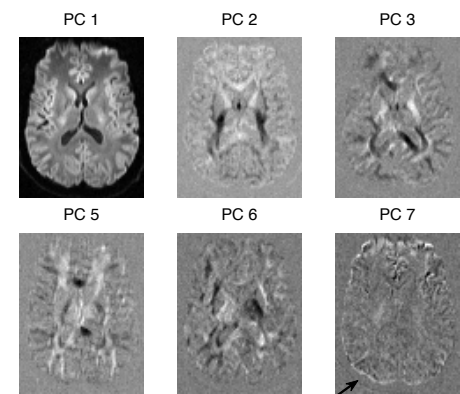

PC 9
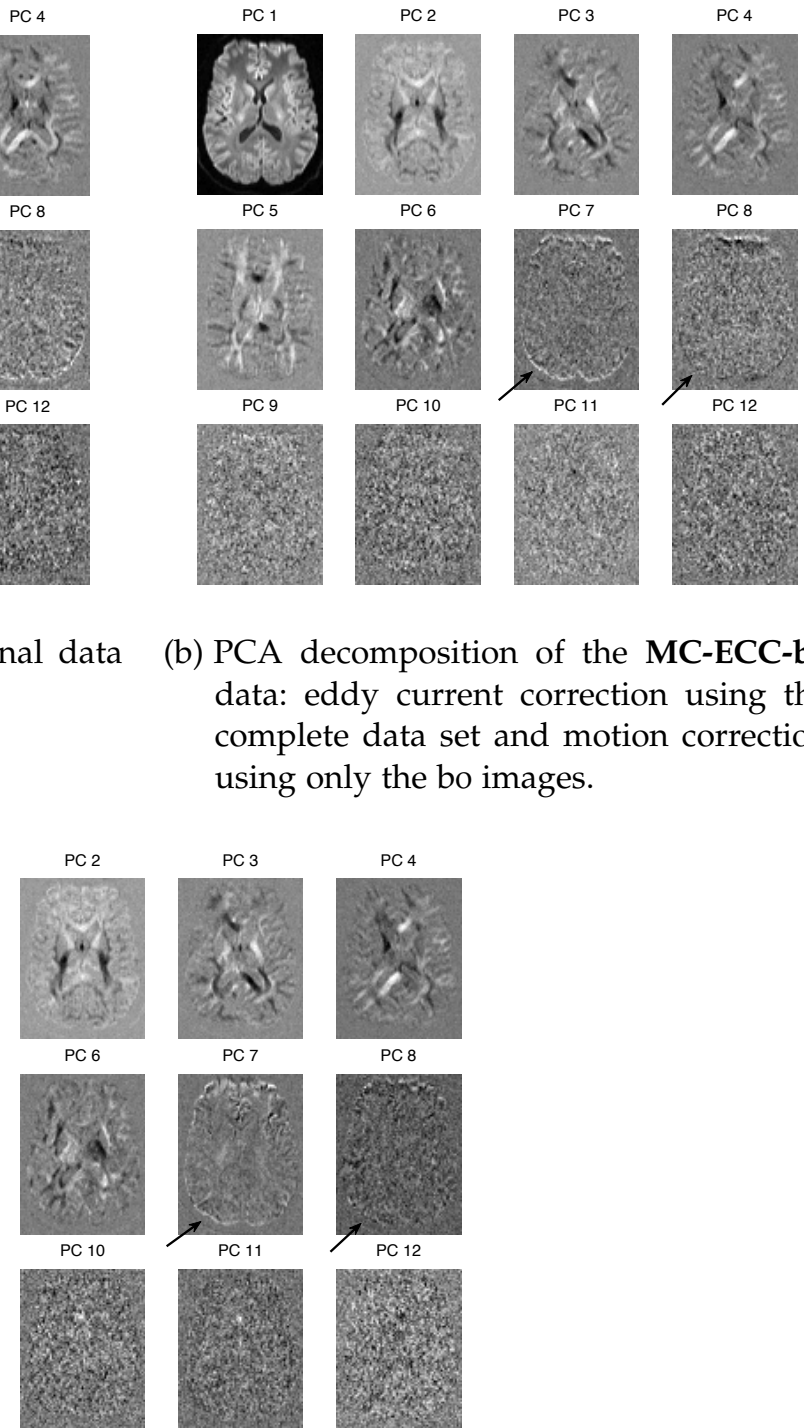

$\mathrm{PC} 7$

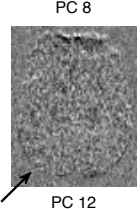

PC 9

PC 10

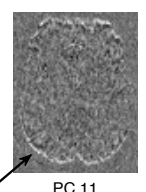

PC 11
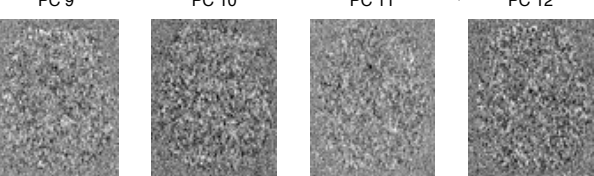

(b) PCA decomposition of the MC-ECC-bo data: eddy current correction using the complete data set and motion correction using only the bo images.
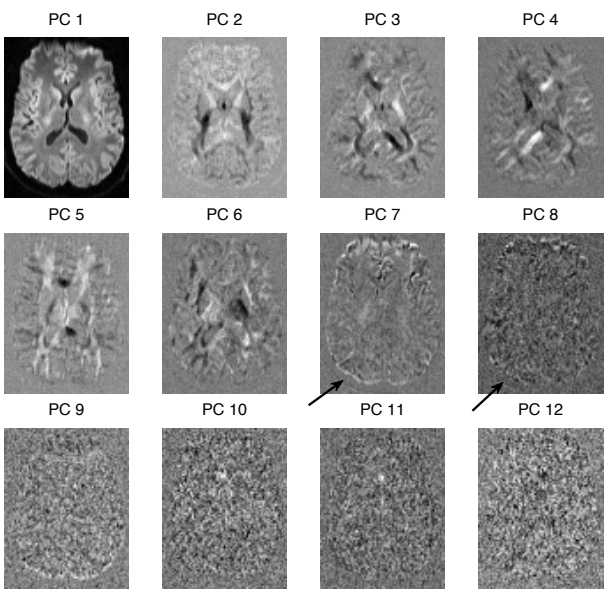

(c) PCA decomposition of the MC-ECC-CM data: motion and eddy current correction using the complete data set.

Figure 4.2: First 12 PCA components (PC) in data set A, slice 46 of a) the original data, b) data corrected for motion with the bo volumes and corrected for eddy currents using the complete data set and c) data corrected for motion and eddy currents using the complete data set.

\subsubsection{Standard deviation across b0 and DWI images}

Standard deviation maps were calculated for the uncorrected data, MC-bo, MCECC-bo and FSL, across $b 0$ images $\left(S_{b 0}\right)$ and across diffusion weighted images $\left(S_{D W I}\right)$. In the uncorrected data in figure 4.4 there are posterior and anterior re- 


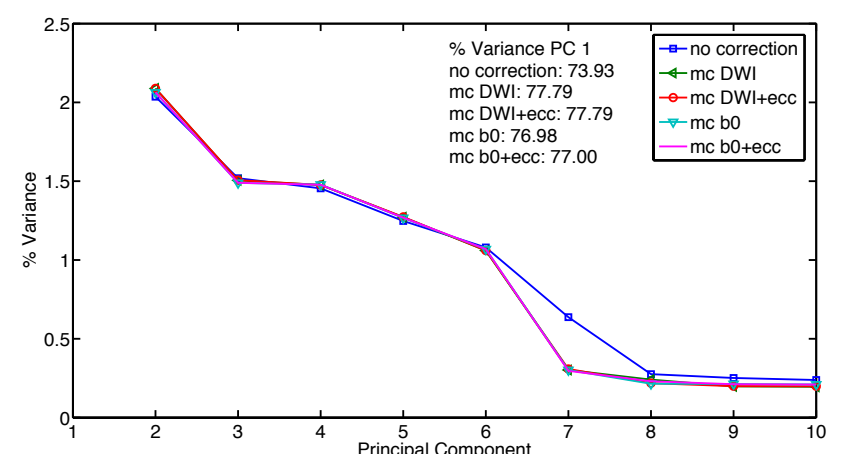

(a) Variance of the first 10 components. MC DWI: motion correction using the complete data set. $\mathrm{MC}$ bo: motion correction using the bos. ECC: eddy current correction. The increase in registration accuracy is most clear in PC 7 and higher. Motion correction using the complete data set is slightly better than motion correction using the bo volumes as revealed by the contribution of the first principal component. Eddy Current correction does not reduce PC significantly, but if we look at figure b), it does improve mis-registration errors in PC 8.

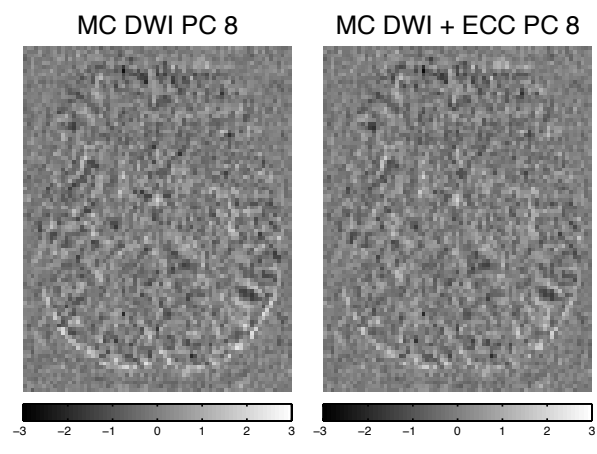

(b) Improvement of the registration by removing the bright edge in the lower part of the brain as shown in PC 8 by the eddy current correction step. Figure intensities are equally scaled.

Figure 4.3: Left: \% Contribution of PCA variance. Right: although not visible as PCA variance decrease in the left figure, eddy current correction increases registration accuracy in PC 8.

gions showing high $S_{b} 0$ (yellow and white colors), as well as increased $S_{b 0}$ in the ventricles. After motion correction $\mathrm{MC}-\mathrm{bo}, S_{b 0}$ decreases, indicating that registration across $b 0$ images has improved. In MC-ECC-bo there is no improvement to be observed because the $b 0$ images are not used for the eddy current correction step. The FSL $S_{b 0}$ images show slightly higher values in the CSF and temporal regions (see arrows).

In the $S_{D W I}$ maps a reduction in $S_{D W I}$ can be observed in frontal regions, see arrows in figure 4.5. The MC-bo method reduces the standard deviation already and there is little visible difference between MC-bo and MC-ECC-bo. There is however a visible difference between MC-ECC-bo and FSL, see the box in figure 4.5. The FSL image shows an overall higher $S_{D W I}$ than our implementation.

\subsection{DISCUSSION}

We propose a method to iteratively correct for eddy current-induced distortions and bulk motion in DW-MRI data. Correction for bulk motion and EC distortions is a crucial pre-processing step in DW-MRI analysis, because later compu- 


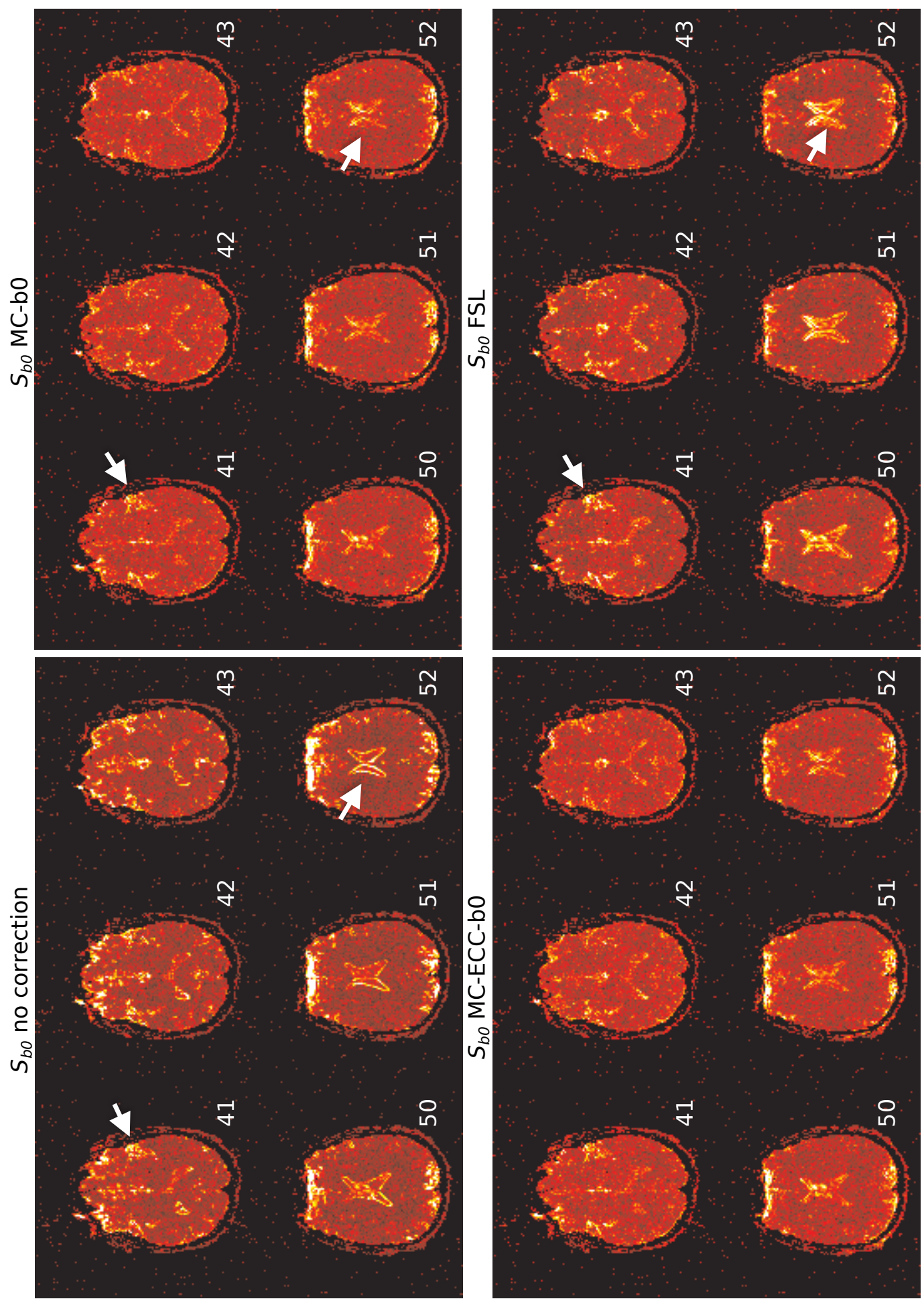

Figure 4.4: Standard deviation across bo images. Red colors: low $S_{b 0}$, yellow-white colors: high $S_{b 0}$. Arrows indicate the changes and differences between methods, see text. 

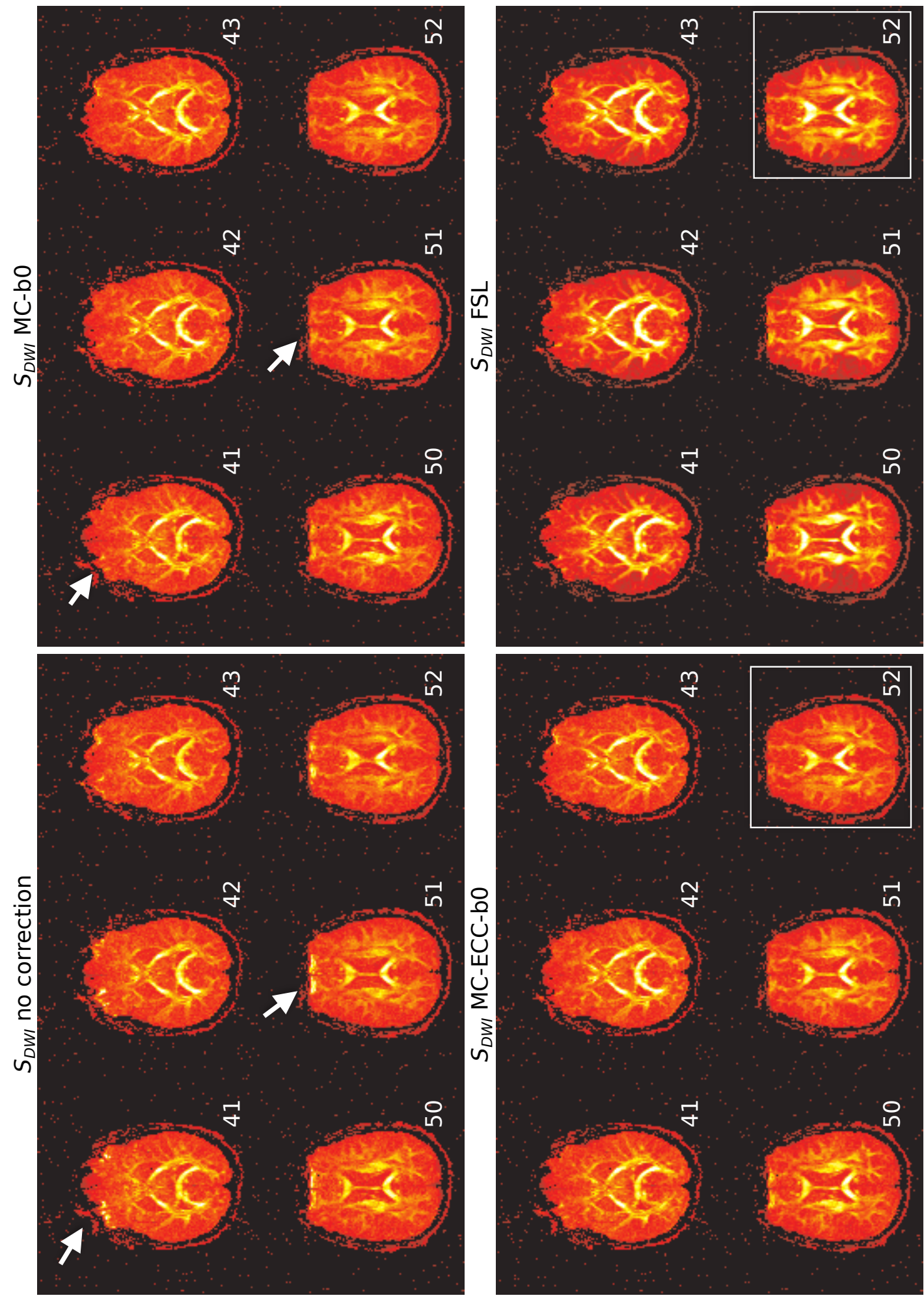

Figure 4.5: Standard deviation across DWI images. Red colors: low $S_{b 0}$, yellow-white colors: high $S_{b 0}$. Arrows and the boxes indicate the changes and differences between methods, see text. 


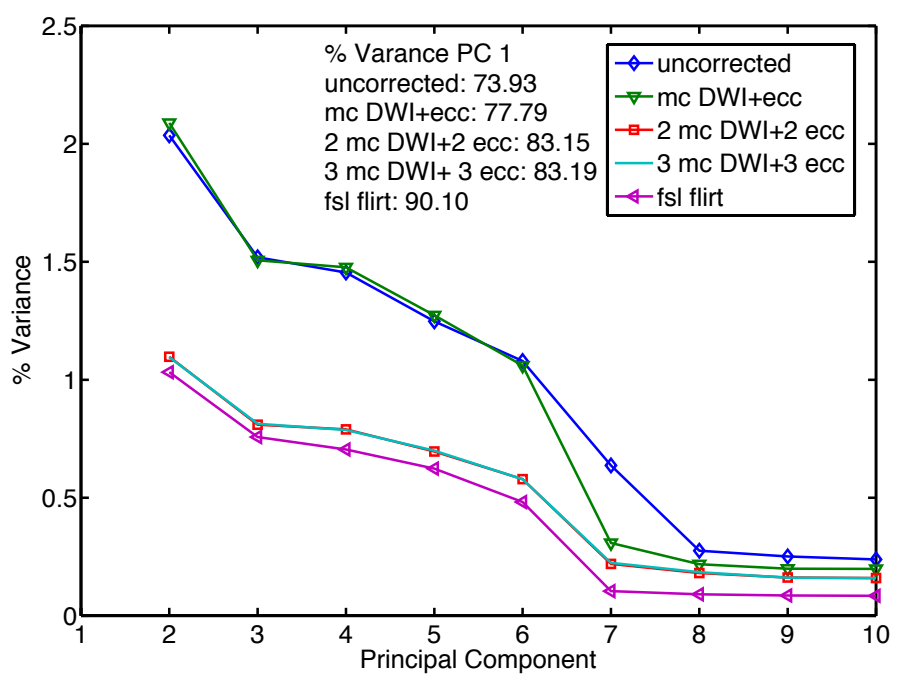

(a) Iterative correction. For two iterations of the algorithm using the complete data set (' $\left.2 \mathrm{mc} \mathrm{DWI}+2 \mathrm{ecc}^{\prime}\right)$, the variance of the first PC is significantly higher and the variance of the second and higher components is lower than when only one iteration is used. A third iteration (' $3 \mathrm{mc}$ DWI+3 ecc') does not further improve registration accuracy. The data set corrected in FSL shows a higher percentage of variance in the first component and lower percent of variance in the 2nd and higher components.

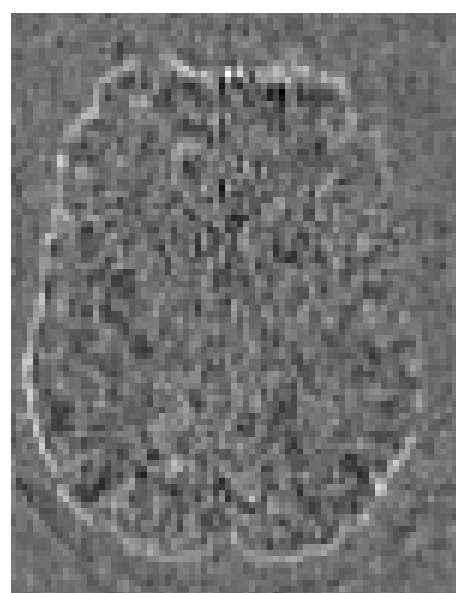

(b) FSL overestimation of the distortions as revealed by a hyper-intense edge in PC 7 .

Figure 4.6: Iterative correction for motion and eddy currents and eddy current correction in FSL.

tations on the data rely on and assume perfect registration across the imaging volumes. We used the normalized gradient field method [7] as a motion correction procedure, and "brute force" cross-correlation and correction for the diffusion gradient direction [3] of a reference volume and a distorted volume to find translation, shear and scaling in individual slices. This combination of methods optimally uses prior knowledge to usefully constrain the various detection and correction steps. EC distortion is corrected per slice with an affine transformation (translation, scaling and shear), whereas motion is corrected in $3 \mathrm{D}$ over the whole volume with a rigid body transformation. We have found that using the complete DW-MRI dataset improves registration as compared to using only the bo images for estimation of motion correction parameters. Moreover, by iterating the combination of these two steps they mutually improve each other's results, as shown by continued improvement of the alignment of the first iteration in the second iteration.

Affine $3 \mathrm{D}$ registration methods, such as FSL-FLIRT, which register the full DW volumes to the bo volume, tend to overestimate distortions in the read-out direction of the image [5]. This is a consequence of i) the lack of constraints on the registration and ii) the correction of a slice-based EC distortion by a 
global volume transformation. The cross-correlation method by Haselgrove [6] does not suffer from this problem, since distortions are only estimated in the phase-encoding direction of individual slices. However, this method fails when bulk-motion has put slices in the reference bo volume and DW volumes out of alignment and the original algorithm depends on phantom calibration methods, which need to be redone when the scanner hardware or diffusion MRI sequence have changed. The proposed MC-ECC procedure avoids both these problems by its two step iterative approach and the implementation of diffusion gradient modelling of the distortions and thus achieves superior distortion correction and alignment.

Previously a quantitative comparison of the ICC method with phantom calibration and FSL's eddy current correction was made [5]. In this investigation, the reduction of PCA variance in the second and higher components was larger for the ICC method then for FSL-FLIRT, indicating a better performance of the ICC method with phantom calibration. In the method as we have implemented it, the percentage of variance in the and and higher PC components was slightly higher than in FSL-FLIRT. However, FSL-FLIRT does show an overestimation of the distortions, while our method does not.

The standard deviations across $b 0$ and DWI images can only be used quantitatively. By eyeballing the $S_{b 0}$ and $S_{D W I}$ images, the improvement because of motion correction can be clearly seen and small but detectable differences between FSL and our method are visible.

In contradistinction to the phantom calibration based implementation of the ICC algorithm, our method is more generally usable as it can be used on every data set already acquired.

In conclusion, we have implemented a fast algorithm to correct for eddy currents and motion in DW-MRI data. The method can be run iteratively if needed, thereby optimizing the registration quality. Finally, the method can be used on any acquired DW-MRI data set without the need for phantom calibration.

\section{REFERENCES}

[1] D Le Bihan, C Poupon, A Amadon, and F Lethimonnier. Artifacts and pitfalls in diffusion MRI. Journal of Magnetic Resonance Imaging, 24(3):478$88,2006$.

[2] TG Reese, O Heid, RM Weisskoff, and VJ Wedeen. Reduction of EddyCurrent-Induced Distortion in Diffusion MRI Using a Twice-Refocused Spin Echo. Magnetic Resonance in Medicine, 182:177-182, 2003.

[3] J Zhuang, J Hrabe, A Kangarlu, D Xu, R Bansal, CA Branch, and BS Peterson. Correction of Eddy-Current Distortions in Diffusion Tensor Images 
Using the Known Directions and Strengths of Diffusion Gradients. Journal of Magnetic Resonance Imaging, 1193:1188 -1193, 2006.

[4] SM Smith et al. Advances in Functional and Structural MR Image Analysis and Implementation as FSL. NeuroImage, 23(S1):208-219, 2004.

[5] S Muñoz Maniega, ME Bastin, and PA Armitage. A quantitative comparison of two methods to correct eddy current-induced distortions in DT-MRI. Magn Reson Imaging, 25(3):341-9, 2007.

[6] JC Haselgrove and JR Moore. Correction for distortion of echo-planar images used to calculate the apparent diffusion coefficient. Magnetic Resonance in Medicine, 36(6):960-964, December 1996.

[7] E Haber and J Modersitzki. Intensity gradient based registration and fusion of multi-modal images. Med Image Comput Comput Assist Interv., 9(Pt 2):726$733,2006$.

[8] BM Ter Haar Romeny. Front-End Vision and Multi-Scale Image AnalysisMulti-Scale Computer Vision Theory and Applications, Written in Mathematica. Springer, 2003.

[9] ME Bastin. Correction of eddy current-induced artefacts in diffusion tensor imaging using iterative cross-correlation. Magnetic Resonance Imaging, 17(7):1011-24, 1999.

[10] A Leemans and DK Jones. The B-matrix must be rotated when correcting for subject motion in DTI data. Magnetic Resonance in Medicine, 61(6):133649, 2009.

[11] GK Rohde, AS Barnett, PJ Basser, S Marenco, and Cs Pierpaoli. Comprehensive approach for correction of motion and distortion in diffusion-weighted MRI. Magnetic Resonance in Medicine, 51(1):103-114, 2004.

[12] J-D Tournier, S Mori, and A Leemans. Diffusion tensor imaging and beyond. Magn Reson Med, 2011. 

Part II APPLICATIONS OF DW-MRI 

LETTER-SOUND INTEGRATION IN RECOVERED

LANDAU-KLEFFNER SYNDROME: AN FMRI CASE STUDY

this Chapter is baSed on Pullens P, Pullens W, Blau V, Sorger B, Jansma B and Goebel R. Letter-sound integration in recovered Landau-Kleffner Syndrome: an fMRI case study under review. 


\section{ABSTRACT}

Landau-Kleffner Syndrome (LKS) is a rare form of acquired aphasia in children, characterized by epilepsy, mostly during sleep, and epileptiform discharges in the electroencephalogram. After initial normal speech and language development, aphasia develops at age of 3 to 7 , in a period of days to months. After the active epileptic phase, which usually ends when patients reaches adulthood, language outcome is usually poor if no treatment focused on restoration of communication is given. Patients often appear deaf-mute, but auditory information is still processed up to the primary auditory cortex. Sign Language, as part of the treatment, may lead to recovery of communication.

The neural mechanisms underlying poor language outcome in LKS are not yet understood. It is hypothesized that the deficit underlying LKS is generalized auditory amnesia instead of a phonological decoding deficiency, so an impairment in the auditory circuit might exist. In this first detailed functional MRI study audiovisual multi-sensory processing was investigated, since LKS patients are often proficient in reading, but not in speech perception.

In the recovered LKS patient a striking large difference in the cortical area activation to auditory stimuli was found in the left versus the right auditory hemisphere, which cannot be attributed to hearing loss. Compared to healthy proficient readers investigated earlier with the same fMRI experiment, lettersound integration in the patient resembles normal letter-sound integration in the superior temporal gyrus as demonstrated by the multi-sensory interaction index, indicating that language deficits in LKS occur in higher regions responsible for word and sentence processing.

\subsection{INTRODUCTION}

\section{Landau-Kleffner Syndrome}

Landau-Kleffner Syndrome (LKS) was first described in 1957 [1]. It is a form of acquired auditory aphasia in children, characterized by either partial or total loss of auditory comprehension or environmental sounds [2]. After initial typical speech and language development, children with LKS develop (semi-) acute aphasia in a period of days to months at the age of three to seven years. Aphasia is mostly receptive (phonological decoding) and develops into expressive impairments at a later stage [3].

Auditory agnosia, i.e. the inability to distinguish sounds is also reported in LKS. While it is unclear whether this inability to distinguish sounds reflects a specific phonological decoding deficiency or a generalized form of auditory amnesia, ample data support the hypothesis that symptoms of LKS are related toan impairment in the auditory cortical processing circuitry [4]. 
Shortly before or after the onset of aphasia, symptoms of epilepsy start to occur. These may be expressed by visible epileptic seizures, but are mostly only visible as epileptiform discharges in the EEG over bi-temporal, tempo-parietal and parieto-occipital regions [5]. Patients suffer from paroxysmal uni-or bilateral spike-and-wave discharges with a maximum over temporal regions, that become almost continuous during slow-wave sleep [2]. The severity of this seizure activity is variable and most symptoms tend to disappear when the patients enter adolescence.

In addition, and as a result of the epileptic seizures and possibly associated treatment, other higher-order cortical functions such as intelligence and nonverbal learning ability can be affected as well. These cognitive dysfunctions are often associated with dramatic changes on the behavioural level. Two-thirds of the studied LKS patients show signs of behavioural change including aggression, attention and concentration disorders, as well as autism-related behaviours such as resistance against change, echolalia, echopraxia, and even psychotic behaviours [6]. It remains unclear whether these behavioural changes are caused by the severe lack of communication and/or have neurological underpinnings. When treatment focussed on any form (auditory, visual) of communication is offered to a patient, a reduction of problematic behaviour is observed $[7,8]$.

When symptoms of LKS first arise, the patient seems to be deaf, but audiograms are usually normal [5]. In contrast to electrophysiological measures, the structural MRI typically does not show abnormalities [9].

LKS Treatment commonly consists of anti-epileptic drugs or corticosteroids. Neurosurgery in form of multiple subpial transection [10], ketogen-diet combined with treatment focussed on improving communication is also applied [11]. However, in all of these treatments, prognosis is hard to determine and full recovery is unlikely. Whereas some patients may have permanent language disorder, some regain much of their language capabilities.

\section{Neural Mechanisms underlying LKS}

Research into the mechanisms underlying the LKS syndrome is mostly done on patients during the active epileptic phase. EEG studies have shown that auditory information (speech and natural sounds) is processed normally up to the primary auditory cortex [2]. As for the higher level in the auditory processing stream however, LKS patients in the active and recovered phase suffer from permanent dysfunction of associative auditory cortex as measured in a dichotic listening task during EEG recordings [12, 13, 14]. This dysfunction is expressed as a unilateral dichotic extinction, contralaterally to the epileptic focus [12]. Furthermore, short-term phonological memory is consistently found to be impaired in LKS, even in patients who have recovered fairly well [15]. A positron emission tomography (PET) study (short-term memory word-recalling task) in patients 
six to ten years after the active phase has demonstrated long-lasting dysfunction of superior-temporal gyri and perisylvian areas [15]. Similarly, a PET study in active-phase patient has shown that the tip of the temporal lobe is dysfunctional in LKS patients, and the epileptic discharges possibly affects nearby temporal regions [16].

Importantly, a failure to transmit incoming auditory information beyond early sensory cortices in the temporal lobe leaves LKS patients unable to access meaning from spoken words. Nevertheless, many of the patients with LKS show astonishing recovery of communication using alternate means, such as non-verbal signing. Since LKS has its onset during a critical period of language acquisition, the brain still shows a remarkable ability for plastic change. The critical question therefore is: what is the neural mechanism that allows LKS patients to compensate for their sudden loss of language functions? If the typical route to access meaning about objects/events via spoken language is blocked, how does the brain accommodate the plastic changes needed to recover communication skills? Since LKS patients are often proficient in reading, one possibility is that the brain creates an alternate pathway for communications through non-verbal modalities such as vision (Deonna et al., 2009). Hence, the goal of the present investigation was to examine the neural substrates of restored communication in LKS using functional magnetic resonance imaging (fMRI).

\section{Integration of letters and speech sounds}

The neural mechanism for integration of speech sounds and written letters has previously been studied in healthy subjects [17] and subjects with developmental dyslexia $[18,19]$. In healthy subjects, auditory cortex and auditory association areas in the superior temporal cortex (STS) have been implicated in the integration of letters and speech sounds. Greater neural responses were observed when the letters and the sounds were matching (congruent) as opposed to mismatching (incongruent), indicating specialized processing for the learned audiovisual association between letters and sounds. In contrast, dyslexic adults and children have been shown to be impaired in their integration of letters and speech sounds in those same temporal brain regions $[18,19]$.

\section{Aim of this study}

We used functional magnetic resonance imaging (fMRI) to study the case of an adult female LKS patient whose communication abilities were largely restored following long-term multi-sensory intervention training (see case description). In contrast to previous neuroimaging studies, which have focussed on studying the processing of auditory information, the present study simultaneously examined auditory, visual and audiovisual processing abilities in LKS using the smallest 
possible units of spoken and written language (speech sounds and letters). This allowed us to directly examine the basic compensatory cortical network that may enable visual forms of communication in LKS, such as signing or finger-spelling, and its interaction with previously-established auditory dysfunctions.

\subsection{CASE DESCRIPTION}

We present a case of a 27-year old female, diagnosed with LKS. At present she lives a full live as an educated married woman with a full time job. In the cause of this investigation she had an audiometric evaluation (Viataal Audiometric Centre NL 2009), her audiogram was found to be in a normal range.

\section{Early Childhood}

She was born from a full-term gestation with uncomplicated pregnancy and delivery. First development was normal. At the age of 4 , her parents and the preschool teacher observed problems in language comprehension. At the time, she was perceived to suffer from progressive hearing loss. Neurological examination ( 24 hour EEG) lead to the diagnose Landau-Kleffner Syndrome. At the age of five years, language comprehension and language production became increasingly disrupted. A year later, she was examined by a neuropsychologist, who did not observe any cognitive or visuo-motor impairments. However her speech-language disorders seemed to be caused by sensory aphasia. Additionally, language perception was severely disrupted on a word/sentence level and she was diagnosed with auditory agnosia and jargon-aphasia. Specifically, the neuropsychologist diagnosed a mixed-type aphasia, which is not solemnly caused by auditory agnosia, combined with a very weak auditory memory. Expressive language was severely impaired as well as was the abstraction of inner language. Memory for rhythm was pathologically weak, as were fine motor skills and memory for order of motor tasks. No evidence for dyspraxia was reported, but there was a highly related pattern. Visual memory was strong, she had developed a coding strategy for communication. Lastly, non-verbal abstraction was weak and hence dys-symbolic problems were to be expected.

At the age of seven, she was tested by a psychologist and a speech-therapist. On the executive part of an intelligence test, she scored in the average to aboveaverage (IQ 115). Performance on visual memory was equal or above average compared to her age group. In a non-verbal auditory discrimination task, five out of ten sounds were classified correctly. Spontaneous speech was characterised by jargon-aphasia. Some meaningful two- and multiple-word sentences are produced, at the articulation test, almost all words were correctly pronounced. Errors consisted of word reduction. However, she was severely impaired on audi- 
tory discrimination and, comprehension of sentence structure, fluency of speech and auditory memory.

A second investigation by a psychologist, a month later, showed poor language development: rhythmic jargon including some recognizable words, often a correct vowel, combined with a consonant. Comprehension of spoken language was very limited and occurred only when enough cues were presented. Speechreading was difficult. Learning conditions speech and language comprehension: results for sequential tasks were better than $t-6$ months at the Kaufmann Hand Movements test[20]. Results based on rhythm indicate, again, dyspraxia. Melody and rhythm have been kept, jargon is melodious. She was communicative, and focussed on the face to obtain additional information. However, it was not to be expected at this point in time that oral communication would be possible. Learning conditions written language: results indicate positive skills with respect to inner language, concept forming and symbolism. She had a good visual memory and developed some forms of abstraction. The strategy she used and the mistakes she made did not point to a visual imprinting strategy. Based on these investigations, she was admitted into a specialized school for the deaf. The school curriculum, developed for deaf children with learning disabilities is based on visualised and written conversation and the use of finger spelling. In the first evaluation,the Kaufmann Intelligence Test [20] indicates that she fits the profile of the school's pupils. Conditions for language development were good. Her learning conditions for oral language, however, were so weak that communication by speech was not possible during this time. Simultaneous memory was strong and written language was predicted to play an important role in language development. Successive memory was weak causing problems when reading finger-spelled language. However, after an introduction period active use of finger-spelling was possible. Understanding spoken language was poor; almost no words were being recognized based on hearing only.

\section{School life}

At the age of 13 she started secondary schooling at a school for children with specific language impairment (SLI). After an intermediate year in practice based education she reached a level sufficient to start high-school.

Hereafter she was educated as a teaching assistant on Dutch MBO level (comparable US Associate Degree) in a regular institute. She passed the theoretical part without problems given that reading materials were available. Oral instructions/teaching were only possible given that the teacher made eye-contact with her. It turned out to be impossible for her to do a standard internship as teaching assistant in a classroom, since she could only understand children when they made eye-contact. In a group, she was found to be unable to understand a child and to select a single voice in a group. Therefore, she did an internship in a 
school for children with severe communication disorders, which she completed successfully. Recently (2009), she was awarded a bachelor's degree as a teaching assistant.

\section{Present}

At present, she has a full-time job as a teaching assistant at a school for the deaf. In order to fully understand colleagues and children, she still needs to make eye contact. She continues to make mistakes when speaking, in word form, sentence construction as well as melody and accent. During work-related meetings, a strict communication policy is used: each speaker has to indicate when he/she is speaking and speakers have to speak in concession, not simultaneously. In bigger meetings, a writing interpreter is used in order to help her understand the Dutch language in full.

School practitioners developed the hypothesis that her language disorder can be overruled by using the motor system (finger spelling and gestures) instead of verbal auditory input, which is in agreement with positive results obtained when using finger spelling in LKS [8]. In this way spoken language skills were partly restored and understanding of spoken language based on lip reading and gestures became possible. As adults all former students who had followed a similar program, expressed motor involvement -visible hand twitches- while understanding spoken or written language.

Previous single case evaluations indicated the importance of alternative visual communication systems in order to prevent behavioural problems, emotional disturbances and delayed cognitive development [8]. It was stated that the use of Sign Language or signed oral language, including finger spelling, enabled, not only in severe cases of LKS, a successful recovery of communication functions. In this article the following suggestion was made: "Important issues like successful delayed restoration of unused cortical functions or the occasional occurrence of good reading without phonology or the reorganisation of brain functions should be studied in patients with LKS" [8].

\section{$5 \cdot 3$ METHODS}

A 27-year-old female, diagnosed LKS at age five years (see case description), was invited to participate in the experiment, which was voluntary and in accordance with the Maastricht University ethical guidelines. Informed consent was obtained from the subject.

The stimuli and presentation design were adapted from Blau and colleagues [18]. Stimuli were visual letters and auditory speech sounds corresponding to Dutch single letters. Stimuli were presented with Presentation software (Neurobehavioral Systems, Inc.) in blocks corresponding to four experimental condi- 
tions: unisensory letters (L), unisensory speech sounds (S), multisensory congruent LS pairs, and multisensory incongruent LS pairs. One block was $20.8 \mathrm{~s}$ long, divided into four mini-blocks (5.2 s). During multisensory stimulation, stimuli were presented simultaneously. Subject passively listened to and/or viewed the stimuli. The experiment included four experimental runs, each composed of eight blocks and alternating fixation periods. Each condition (40 trials) was repeated twice per run. The order of blocks was pseudo-randomized within runs, and the order of runs was counterbalanced across subjects.

The experiment was explained and guided by her former teacher and present colleague using speech and finger spelling, to make sure that there were no communication problems while instructing the subject.

MR imaging was performed on a $3 \mathrm{~T}$ Allegra MR scanner (Siemens, Erlangen, Germany) using a birdcage single coil. Blood Oxygenation Level-Dependent (BOLD) signal was acquired with a $\mathrm{T}_{2}{ }^{*}$ sensitive Echo-Planar Imaging (EPI) sequence and $\mathrm{TR} / \mathrm{TE}=5200 \mathrm{~ms} / 30 \mathrm{~ms}$. We acquired $244.5 \mathrm{~mm}$ thick slices covering the whole brain, in a $64 \times 64$ matrix with Field of View (FOV) $192 \times 192$, resulting in $3 \times 3 \times 4.5 \mathrm{~mm}$ voxels. The volume acquisiton time was $1500 \mathrm{~ms}$, followed by a silent period of $3700 \mathrm{~ms}$, during which stimuli were presented. Additionally, a high quality $\mathrm{T}_{1}$ weighted anatomical image was acquired using an ADNI MPRAGE sequence (TR/TE 2250/2.6 ms) with 192 slices and a matrix of $256 \times 256$. The FOV was $256 \times 256 \mathrm{~mm}$, resulting in $1 \times 1 \times 1 \mathrm{~mm}$ voxels.

Data analysis was performed in BrainVoyagerQX 2.1 (Brain Innovation BV, Maastricht, NL). Functional data were first corrected for slice scan time differences, subject motion artefacts, linear drifts and low-frequency drifts using default settings. The fMRI data were then coregistered to the anatomical data and converted into standard Talairach space. Due to severe artefacts, the third run had to be discarded from the fMRI data set. Statistical analysis on fMRI data were performed for the remaining three runs by computing a fixed-effects (FFX) general linear model (GLM) normalized by the z-transform. To correct for multiple comparisons, we estimated a cluster threshold according to the method described in [21] at a $p$-value of 0.005 , which reduces the probability of type II errors commonly found when using the False Discovery Rate [22]. The cluster threshold was estimated at 3 voxels $\left(81 \mathrm{~mm}^{3}\right)$. Percent BOLD signal change was computed by normalizing the predictors to 1 and performing a GLM with percent transform. The extracted BOLD response in selected Regions of Interest (ROIs) was further processed in Matlab R2007b (The Mathworks, USA) using the BVQXtools toolbox, available on htpp: //wiki . brainvoyager. com/BVQXtools. Because of non-normality of the data (tested with a probability plot using the normplot function in Matlab), statistical tests on the \% BOLD signal change were done by a Wilcoxon rank sum test for equal medians, with $p=0.05$. 
Table 5.1: Regions activated by the unisensory conditions with $p<0.005$ and cluster size 3 voxels

\begin{tabular}{|c|c|c|c|c|c|c|}
\hline \multicolumn{7}{|c|}{ Left Hemisphere } \\
\hline \multirow[t]{2}{*}{ Region } & \multicolumn{3}{|c|}{ Center of gravity } & \multirow[t]{2}{*}{ Area } & \multirow[t]{2}{*}{ stim } & \multirow[t]{2}{*}{ size } \\
\hline & $x$ & $y$ & $z$ & & & \\
\hline Cuneus & -24 & -70 & 17 & BA 18 & VIS & 196 \\
\hline Fusiform Gyrus & -43 & -64 & -14 & BA 37 & VIS & 2992 \\
\hline Inferior Occipital Gyrus & -25 & -90 & $-3 \cdot 3$ & BA 18 & VIS & 271 \\
\hline Medial Frontal Gyrus & $-3 \cdot 4$ & -6.3 & 53 & BA 6 & AUD & 83 \\
\hline Middle Occipital Gyrus & -31 & -82 & 6.9 & BA 19 & VIS & 87 \\
\hline Precentral Gyrus & -37 & -12 & 41 & BA 6 & VIS & 112 \\
\hline Precentral Gyrus & -49 & -10 & 46 & $\mathrm{BA}_{4}$ & AUD & 369 \\
\hline Precentral Gyrus & -54 & -6 & 23 & $\mathrm{BA}_{4}$ & AUD & 117 \\
\hline Superior Temporal Gyrus & -35 & -35 & 15 & $\mathrm{BA}_{41}$ & AUD & 132 \\
\hline Superior Temporal Gyrus & -47 & -25 & 6.9 & $\mathrm{BA}_{41}$ & AUD & 305 \\
\hline Superior Temporal Gyrus & -50 & -41 & 16 & BA 13 & AUD & 134 \\
\hline Superior Temporal Gyrus & -62 & -31 & 8.3 & $\mathrm{BA}_{42}$ & AUD & 146 \\
\hline \multicolumn{7}{|c|}{ Right Hemisphere } \\
\hline Region & \multicolumn{3}{|c|}{ Center of gravity } & Area & stim & size \\
\hline & $x$ & $y$ & $z$ & & & \\
\hline Fusiform Gyrus & 43 & -48 & -17 & BA 37 & VIS & 2232 \\
\hline Fusiform Gyrus & 28 & -52 & -13 & BA 37 & VIS & 88 \\
\hline Fusiform Gyrus & 23 & -83 & -19 & BA 19 & VIS & 157 \\
\hline Fusiform Gyrus & 28 & -74 & -23 & BA 19 & VIS & 563 \\
\hline Inferior Occipital Gyrus & 40 & -69 & $-7 \cdot 3$ & BA 19 & VIS & 308 \\
\hline Lingual Gyrus & 27 & -79 & $-4 \cdot 1$ & BA 18 & VIS & 1516 \\
\hline Precentral Gyrus & 47 & $-7 \cdot 5$ & 42 & $\mathrm{BA}_{4}$ & AUD & 330 \\
\hline Precuneus & 26 & -70 & 21 & $\mathrm{BA}_{31}$ & VIS & 741 \\
\hline Precuneus & 24 & -61 & 49 & $\mathrm{BA}_{7}$ & VIS & 138 \\
\hline Superior Temporal Gyrus & 49 & -24 & 7.8 & $\mathrm{BA}_{41}$ & AUD & 2546 \\
\hline Superior Temporal Gyrus & 41 & -32 & 3.1 & $\mathrm{BA}_{41}$ & AUD & 208 \\
\hline
\end{tabular}


From the Talairach normalized anatomical data, cortical reconstructions were created using an automatic cortex reconstruction procedure. The GLM results were projected on the $3 \mathrm{D}$ cortical representation sheet and ROIs were defined on the cortical sheet. Talairach coordinates of significantly active regions were visually inspected and subsequently verified using the Talairach deamon (http: //www. talairach.org/).

\subsection{RESULTS}

\section{Response to unisensory stimuli}

Figure 5.1 shows the brain regions in which neural responses were driven by speech sounds as opposed to visual letters. A more detailed overview of the brain regions is given in Table 5.1. Regions activated by the auditory stimulus include the primary auditory cortex (PT/HG BA 41/42) bilaterally, which shows a remarkable difference in the activation pattern between the right and left hemisphere. The activation in the left hemisphere shows a patch-like pattern, while the activation in the right hemisphere shows a more equally distributed pattern. The size of the right hemispheric activation is significantly larger than the left hemispheric region, see Table 5.1. Additional regions preferentially activated by speech sounds vs. visual letters include the superior temporal sulcus/gyrus (STS/STG, BA 41/42, BA 13) and pre-central gyrus (preCG, Brodmann Area BA 4) bilaterally. In the left hemisphere a region in the precentral gyrus (BA 6) was also activated.

Brain areas that preferentially responded to the presentation of visual letters as opposed to speech sounds include the primary visual cortex, lateral occipital regions BA 18/19, as well as the fusiform gyrus (BA 37 ) bilaterally. In the left hemisphere, a region in the precentral gyrus (BA 6), and two regions in the precuneus (BA 7, 31) were also active in response to the visual stimulus.

We investigated the response in the primary auditory cortex more closely with a ROI analysis. The ROIs encompassed the primary auditory cortex BA 41, center of gravity $x, y, z=(42,-35,14)$ and $(-38,-35,14)$ and visual cortex BA 18 , $x, y, z=(26,-76,-8)$ and $(-35,-81,-8)$. Figure 2 shows the BOLD response in primary auditory and visual cortex to the unimodal stimuli (only visual/only auditory). The primary auditory cortex shows a typical activation BOLD response to the auditory stimulus, while the BOLD response to the visual stimulus did not significantly differ from baseline. In the visual cortex, we observe a response to the visual stimulus, while the response to the auditory stimulus remains at baseline level. Combined with the results of the audiogram (normal range) it can be stated that this subject has the capacity to detect sounds, including vocals and language. 


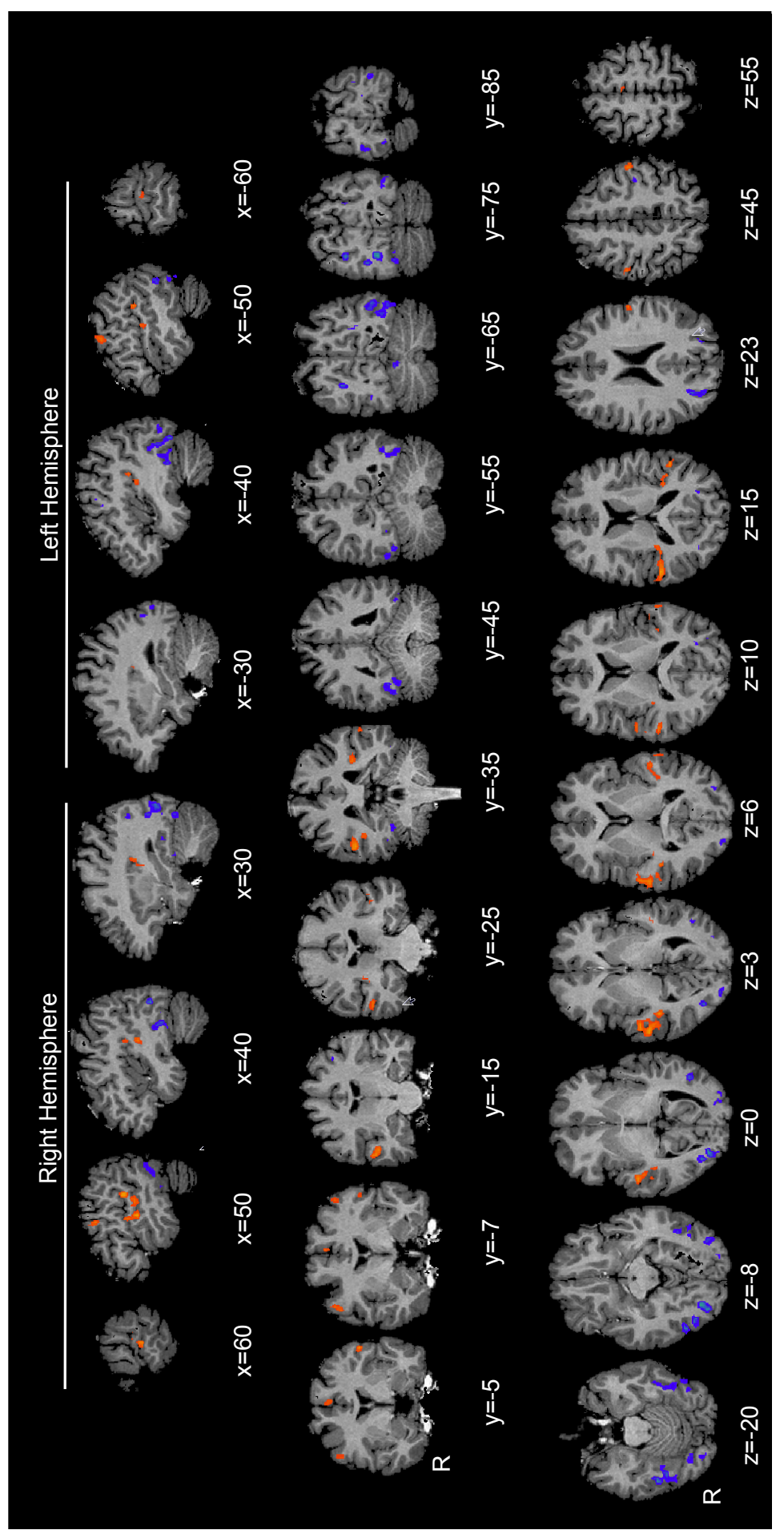

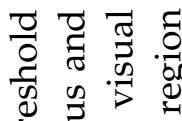

至企

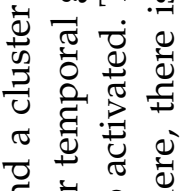

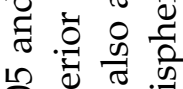

용.

$\checkmark$ 远

$\checkmark$ 永志

動 80

के 오의

売 总

즈류

.

$\wedge$.

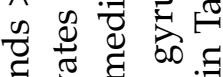

จ․ㄹ छ

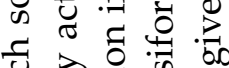

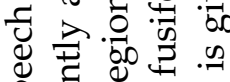

की चु 03

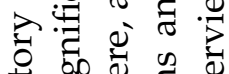

न की

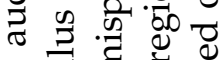

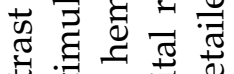

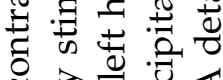

슈ㅇㅝㅠ

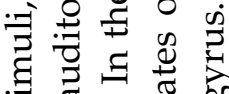

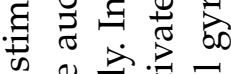

륰휴

焉入艺

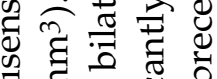

घ 00

히릴

a 0.50 .5

.

: 0 ป

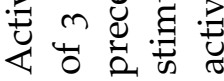

$\ddot{\dot{H}}$

$\overbrace{0}^{0}$ 

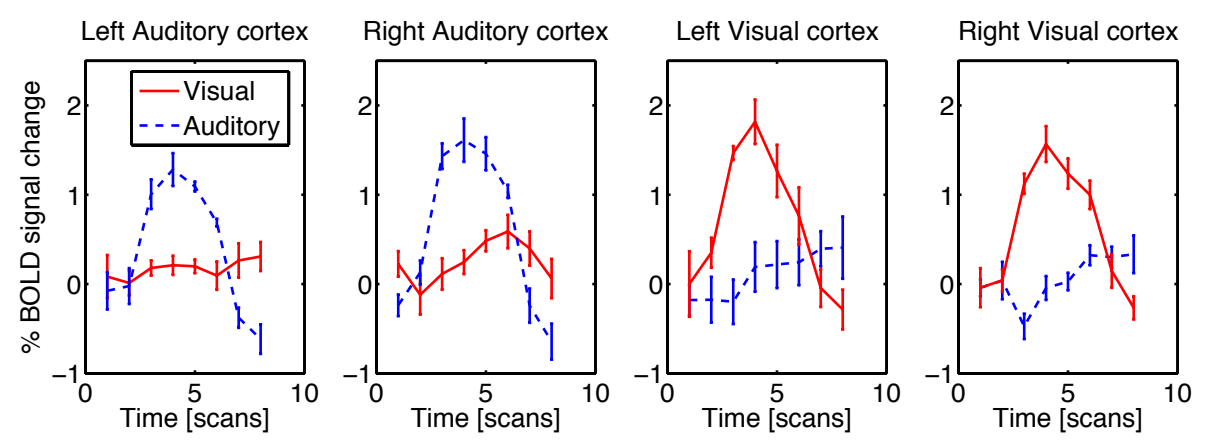

Figure 5.2: BOLD signal change response to unisensory auditory (dashed line) and visual stimuli in primary auditory cortex and visual cortex. In the auditory cortex the response is stronger to the auditory stimulus, while in the visual cortex the response is stronger to the visual stimulus and response to the auditory stimulus stays at baseline level.

In figure 5.3 the relative contribution of auditory and visual stimuli is shown on the inflated cortical sheet. The contribution of the auditory stimulus is shown in red, the contribution of the visual stimulus in green and the areas where both stimuli illicit a response are colored yellow. The response pattern to unisensory auditory and visual stimuly is discussed earlier, so the focus is on the regions that are activated by both unisensory stimuli. In the right hemisphere, these are sup occipital gyrus (associative visual, BA 19), Middle temporal gyrus (BA 21), and superior temporal gyrus STG, as well as cingulate gyrus and sup frontal gyrus (motor planning) on the medial part of the cortex. In the left hemisphere, we also find regions involved in motor planning: medial and middle frontal gyrus, which are activated by both unisensory stimuli.

Since STG is assumed to be involved in integration of letters and sounds [17, 18], we investigated the \% BOLD signal change more closely.

The \%BOLD signal change was extracted from two ROIs, encompassing left and right STG around previously reported [18] coordinates $(-46,-26,6),(45,22,7)$, see figure 5.4a. The ROI was defined on the cortical sheet and projected back onto the $2 \mathrm{D}$ slices. The extracted values are not normally distributed, as assessed by the Matlab normplot command, see figure 5.4b. Therefore, we used a non-parametric Wilcoxon rank sum test for equal medians to infer on differences between the conditions.

Figure 5.5 shows the \%BOLD signal change in the superior temporal gyrus, which is associated with letter sound integration $[17,18]$. Looking at the unisensory stimuli, there is a higher response to the auditory than to the visual stimulus. The spread of BOLD signal change, represented by the 1.5 times interquartile range (whiskers in figure 5.5) in the left hemisphere STG is smaller than in the right STG, which is possibly related to the more concentrated focus of activation (smaller ROI) in the left versus the right STG. When looking at the con- 


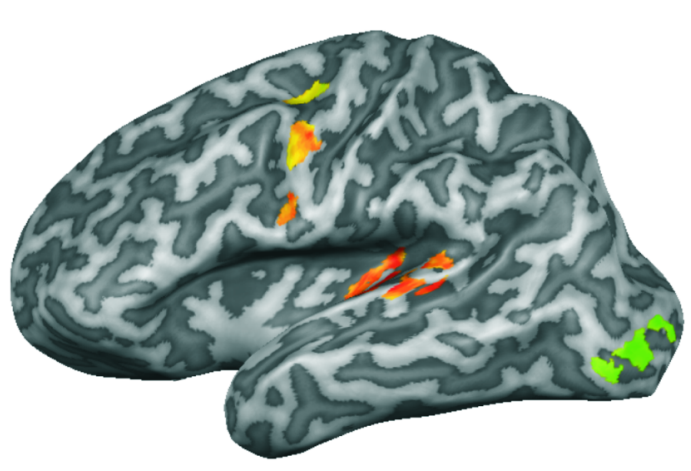

Left hemisphere

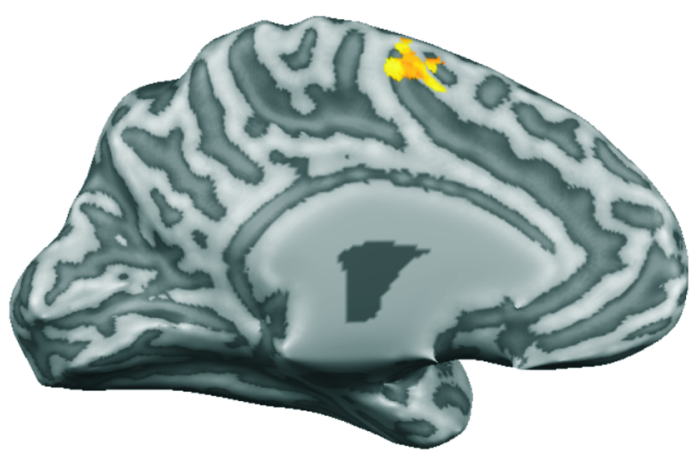

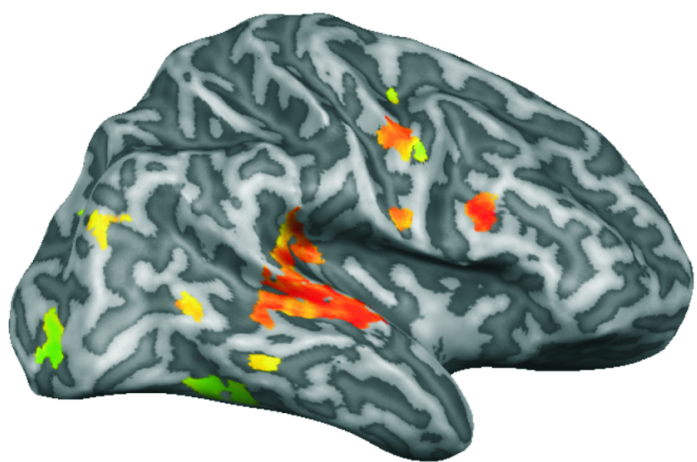

Right hemisphere

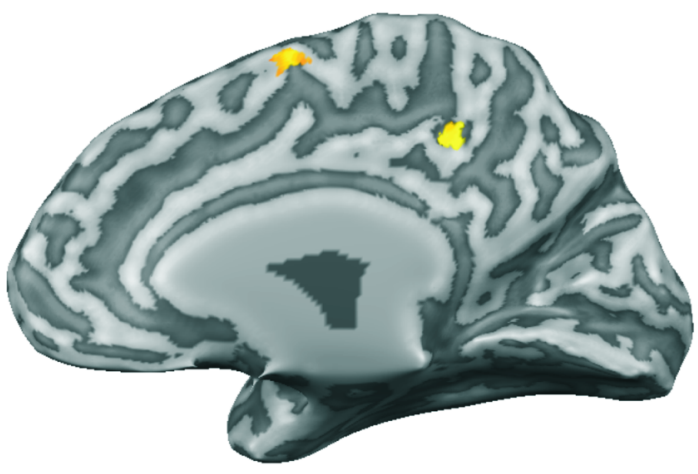

IHIHIHIHIHIH

Auditory
Visual

Figure 5.3: Relative contribution GLM map superimposed on inflated cortical sheet. Color coding of significantly activated regions relative to the presented unisensory stimulus. Red indicates auditory, yellow activation by both stimuli, and green indicates significant activation by visual stimuli. Not only regions related to processing of auditory and visual information are significantly activated, but also regions related to planning of complex hand movements (see text).

gruent versus the incongruent condition; that is, congruent versus incongruent letter-sound pairs, we find that there is a significant higher response (Wilcoxon rank sum test for equal medians, $(p<1 e-30)$ for the congruent condition than for the incongruent letter-sound pair.

The Multisensory Interaction index (MSI) is a measure for the amount of integration of the multisensory stimuli relative to the maximum unisensory response

$$
M S I=\frac{\left(M S-U S_{\max }\right)}{U S_{\max }}
$$

where $M S=$ median value of the multisensory stimulus (= congruent or incongruent stimulus), $U S_{\max }=$ median of the largest unisensory response. A positive MSI value indicates a response enhancement with respect to the unisensory 


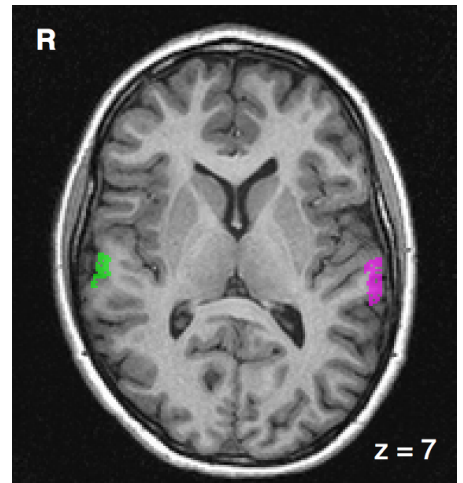

(a) Left and right STG ROI definition. The ROIs were defined around TAL coordinates $(-46,-26,6),(45,22,7)$ on the cortical sheet and projected back onto the $2 \mathrm{D}$ slices. Left STG is 1108 $\mathrm{mm}^{3}$, right STG $894 \mathrm{~mm}^{3}$.
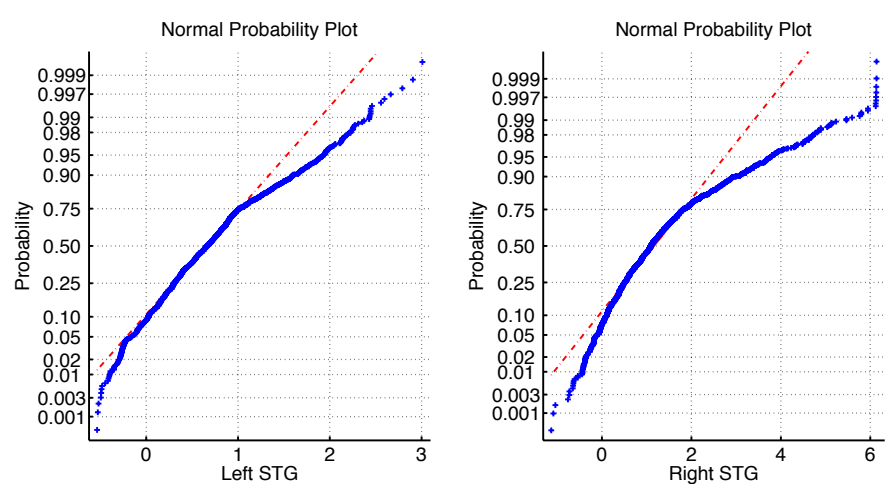

(b) Normal probability plots for \% BOLD signal change for the congruent condition in left and right STG. The data deviates from the straight line and is therefore not normally distributed.

Figure 5.4: STG ROI definition and normal probability plots of the \% BOLD signal change in left and right STG.
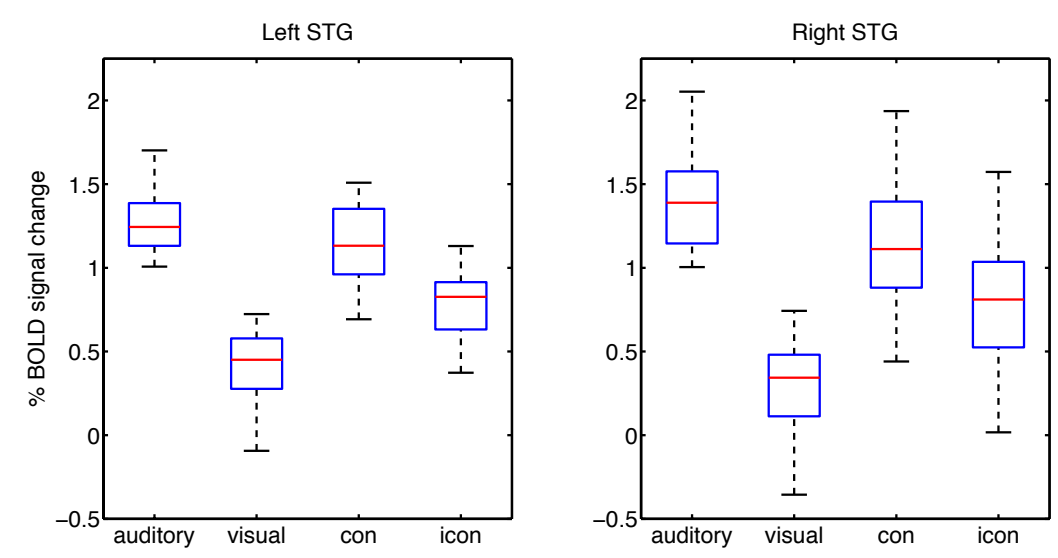

Figure 5.5: Box-and-whisker plot of \%BOLD signal change in the STG. The box is limited by lower, median and upper quartile values, while the whiskers extend to 1.5 times the interquartile range from the ends of the box. The response to congruent vs incongruent letter-sound pairs is significantly different in both hemispheres (Wilcoxon rank sum test for equal medians $p<1 e-30, \alpha=0.05$. 

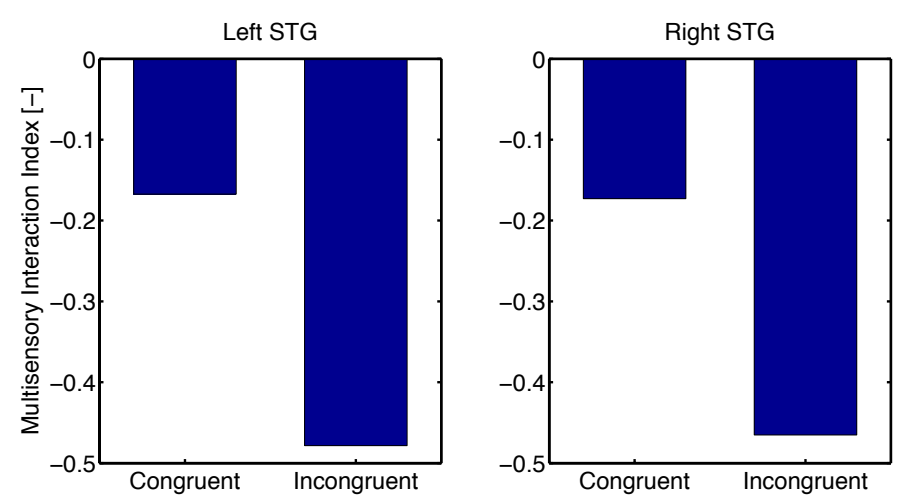

Figure 5.6: Multi Sensory Interaction (MSI). The MSI is different for the congruent vs incongruent condition in both hemispheres, indicating a multi-sensory integration effect.

stimulus, while a negative MSI represents suppression of the response. In figure 5.6, the MSI in the left and right STG (same ROI as used for figure 5.5) for this subject is shown. Bilaterally, the MSI is more negative for the incongruent as for the congruent condition, indicating a modulation of the response, which is possibly representing integration of letters and sounds. Interestingly, this difference in MSI is not found in the right STG, where the MSI is large and negative in both the congruent and incongruent condition.

\section{$5 \cdot 5$ Discussion}

Right lateralized response to auditory speech sounds

In healthy adults, the response to unisensory speech sounds is bilaterally similar and spread out over the PT/HG [17]; We find in the current subject a larger response area in the right hemisphere compared to the left hemisphere, which is reflected in a larger, coherent area of activation $\left(2754 \mathrm{~mm}^{3}\right)$ in the right hemisphere and a more scattered and in total smaller area of activation $\left(583 \mathrm{~mm}^{3}\right)$ in the left hemisphere. The deviating response in the left hemisphere cannot be related to a hearing deficit, since the audiogram is normal and the BOLD response in the primary auditory cortex is comparable to that in healthy subjects studied with the same experiment [17]. A previous single case fMRI experiment, evaluating the response to spoken words in a left-handed post-epileptic phase LKS patient [23] reveiled a similar, although less outstanding, right lateralization: right auditory cortex showed an increase response relative to the left hemisphere, despite the fact that the epileptic foci were on the right side in the reported case.

The BOLD responses to the unisensory stimuli in auditory and visual cortex are in line with results obtained earlier in healthy subjects, indicating that early auditory and visual cortex function normally and are able to relay information 
up to higher cortical areas for further processing. Since the subject is proficient in reading, the processing of visual letters is therefore considered to be effective.

\section{Pre-central gyrus and pre-SMA activation in response to auditory speech sounds}

Activation of pre-motor areas has been reported for speech sound perception [24]. These regions are related to speech production using the mouth and lips and have been found to be robustly activated in healthy subjects while passively listening to meaningless monosyllables [24]. The reported MNI coordinates from left precentral gyrus $(-50,-6,47)$ and right precentral gyrus $(55,-3,45)$ correspond to TAL coordinates: left $(x, y, z)=(-49.5,-3.7,43.5)$, right $(54.5,-1,41.5)$ and overlap the premotor areas in this subject, which are centered around $(-49,-10$, 46) and $(47,-7 \cdot 5,42)$.

Considering the fact that finger spelling is the native language of this subject, it is surprising that the pre-motor areas for finger/hand movements are not recruited in this experiment. The motor areas related to finger movements lie a bit more superior $(-35.5,-14.6,65.3)$, as reported in [25]. Finger-twitching is observed frequently in LKS patients, who use sign language/finger spelling, when listening to spoken language [23] and personal observations with multiple patients (WP). It could therefore very well be that for more complex words and sentences, rather than the single letters presented in the current study, finger spelling or sign language is adopted as an aid to understanding.

\section{Integration of letter-sound pairs in the STG}

In healthy, proficient reading adults, letter-sound integration is found to take place in the STS/STG and the STS/STG responds stronger to the auditory than to the visual stimuli. In our subject, we observe similar behaviour in the STG. When looking at the MSI index, we observe that bilaterally, a modulation of the BOLD response between congruent and incongruent condition is observed, and this is indicative for multi-sensory integration [17, 18]. It might indicate that in LKS, processing of single letters is functioning properly and problems occur at a higher language level.

\section{Conclusions and Hypothesis}

In this study, the integration of visual letters and auditory sounds in a recovered, post-epileptic phase Landau-Kleffner patient was investigated. To our knowledge this is one of the first fMRI studies investigating the underlying mechanisms of LKS at such a fundamental, basic part of language processing, which is vital for day-to-day communication. 
The results from the letter-sound integration in the STG suggest that in recovered LKS, higher language processing up to single letters is probably organised in the same way as in normals, suggesting the language system is still in tact, however not accessible in the same way as in normal adults with full language skills. Problems in understanding language should therefore be located in higher auditory regions, responsible for processing larger language element such as words or sentences. This phenomenon was suggested earlier by [26].

We observe considerable lateralization in the early auditory areas (right lateralized), which was also observed in an earlier study [23]; it remains unknown what causes the lateralization and what cognitive implications it might have.

Although this article is based on fMRI data of a single subject, the outcome seems to be in line with observations and data from researchers in the field of LKS. Until now there was no evidence, but anecdotal, over the use of visual supported communication systems in the treatment of this syndrome. Further investigation on al larger scale, including more subjects and a lager test battery will give us more clarity over the importance of language acquisition through visual information channels.

\section{REFERENCES}

[1] WM Landau and FR Kleffner. Syndrome of acquired aphasia with convulsive disorder in children. Neurology, 7:523-530, 1957.

[2] OK Steinlein. Epilepsy - aphasia syndromes. Expert Rev Neurother, 9(6):825833,2009 .

[3] G Lanzi, P Veggiotti, S Conte, E Partesana, and C Resi. A Correlated Fluctuation of Language and EEG Abnormalities in a Case of the Landau-Kleffner Syndrome. Brain E Development, 16(4):329-334, 1994.

[4] VM Pedro and G Leisman. Hemispheric integrative therapy in LandauKleffner Syndrome: Applications for rehabilitation sciences. International Journal of Neuroscience, 115(8):1227-1238, 2005.

[5] CJ Feekery, B Parryfielder, and IJ Hopkins. Landau-Kleffner Syndrome 6 Patients Including Discordant Monozygotic Twins. Pediatric Neurology, 9(1):49-53, 1993.

[6] A Zivi, G Broussaud, S Daymas, J Hazard, and C Sicard. Epilepsia-acquired aphasia syndrome with psychosis - report of a case. Annales De Pediatrie, 37(6):391-394, 1990.

[7] RE Appleton. The landau-kleffner syndrome. Archives of Disease in Childhood, 72(5):386-387, 1995 . 
[8] T Deonna, AC Prelaz-Girod, C Mayor-Dubois, and E Roulet-Perez. Sign language in Landau-Kleffner syndrome. Epilepsia, 50(suppl 7):77-82, 2009.

[9] M Bureau. Continuous spikes and waves during slow sleep (CSWSS): Definition of a syndrome. In M.B.A. Beaumanoir, T. Deonna, L. Mira, and C. A. Tassinari, editors, Continuous spikes and waves during slow sleep, pages 17-26. Libbey, London, UK, 1995.

[10] K Irwin, J Lees, C Polkey, G Alarcon, C Binnie, M Smedley, G Baird, and RO Robinson. Multiple subpial transection in Landau-Kleffner syndromes. Developmental Medicine and Child Neurology, 43(4):248-252, 2001.

[11] L Lagae. Rational treatment options with AEDs and ketogenic diet in Landau-Kleffner syndrome: still waiting after all these years. Epilepsia, 5o(suppl 7):59-62, 2009.

[12] N Wioland, G Rudolf, and MN Metz-lutz. Electrophysiological evidence of persisting unilateral auditory cortex dysfunction in the late outcome of Landau and Kleffner syndrome. Clin. Neurophysiol., 112:319-323, 2001.

[13] M Plaza, MT Rigoard, C Chevrie-Muller, H Cohen, and A Picard. Shortterm memory impairment and unilateral dichotic listening extinction in a child with Landau-Kleffner syndrome: Auditory or phonological disorder? Brain and Cognition, 46(1-2):235-240, 2001.

[14] MN Metz-Lutz, A de Saint Martin, E Hirsch, P Maquet, and C Marescaux. Impairment in auditory verbal processing and dichotic listening after recovery of epilepsy in Landau and Kleffner syndrome. Brain and Cognition, 40(1):193-197, 1999.

[15] S Majerus, S Laureys, F Collette, G Del Fiore, C Degueldre, A Luxen, M Van Der Linden, P Maquet, and MN Metz-Lutz. Phonological short-term memory networks following recovery from Landau and Kleffner syndrome. Hum. Brain Mapp., 19:133-144, 2003.

[16] H Shiraishi, K Takano, T Shiga, M Okajima, A Sudo, N Asahina, S Kohsaka, M Fukuhara, and S Saitoh. Possible involvement of the tip of temporal lobe in landau-kleffner syndrome. Brain E Development, 29(8):529-533, 2007.

[17] NM Van Atteveldt, E Formisano, R Goebel, and L Blomert. Integration of letters and speech sounds in the human brain. Neuron, 43:271-282, 2004.

[18] VC Blau, NM Van Atteveldt, M Ekkebus, R Goebel, and L Blomert. Reduced neural integration of letters and speech sounds links phonological and reading deficits in adult dyslexia. Current Biology, 19:503-508, 2009. 
[19] V Blau, J Reithler, N Van Atteveldt, J Seitz, P Gerretsen, Goebel R, and Blomert L. Deviant processing of letters and speech sounds as proximate cause of reading failure: a functional magnetic resonance imaging study of dyslexic children. Brain, 133(Pt 3):868-879, 2010.

[20] AS Kaufman and NL Kaufman. Kaufman Test of Educational Achievement: Comprehensive Form manual. American Guidance Service, Circle Pines, MN, 1985.

[21] R Goebel, F Esposito, and E Formisano. Analysis of functional image analysis contest (FIAC) data with Brainvoyager QX: From single-subject to cortically aligned group general linear model analysis and self-organizing group independent component analysis. Human Brain Mapping, 27:392-401, 2006.

[22] MD Lieberman and WA Cunningham. Type I and Type II error concerns in fMRI research: re-balancing the scale. SCAN, 4:423-428, 2009.

[23] JS Sieratzki, GA Calvert, M Brammer, A David, and B Woll. Accessibility of spoken, written, and sign language in landau-kleffner syndrome: a linguistic and functional mri study. Epileptic Disorders, 3(2):79-89, 2001.

[24] S Wilson, A Pinar Saygin, MI Sereno, and M Iacoboni. Listening to speech activates motor areas involved in speech production. Nature Neuroscience, 7(7):701-702, 2004.

[25] CI Moore, CE Stern, S Corkin, B Fischl, AC Gray, BR Rosen, and Dale AM. Segregation of somatosensory activation in the human rolandic cortex using fMRI. J Neurophysiol, 84:558-569, 2000.

[26] MN Metz-Lutz. The assessment of auditory function in CSWS: Lessons from long-term outcome. Epilepsia, 50:73-76, 2009. 

WHITE MATTER LANGUAGE PATHWAYS IN RECOVERED LANDAU-KLEFFNER SYNDROME: A CASE STUDY

this chapter is based on Pim Pullens, Will Pullens, Milene Bonte, Bernadette M Jansma and Rainer Goebel. White matter language pathways in recovered Landau-Kleffner Syndrome: A case study In preparation. 


\section{ABSTRACT}

Landau-Kleffner Syndrome (LKS) is a rare form of acquired aphasia in children due to temporal lobe epilepsia, leading to semi-acute aphasia in a short period of time in the age of 3-10 years old. After the epileptic phase, which usually ends when the child reaches adulthood, severe language deficits remain. The underlying neuronal mechanisms in LKS are not yet understood.

In this paper, the fiber tracts of the language system, the arcuate fasciculi and the fiber tracts originating from the primary auditory cortex were investigated by DTI based fiber tracking in a recovered LKS patient.

The results indicate a rightward lateralisation of the short segments of the arcuate fasciculus and fibers originating from primary auditory cortex. More specifically, we did not find evidence for a connection from the arcuate fasciculus to inferior parietal lobe in the left hemisphere. The inferior parietal lobe, part of the associative cortex, is critical for language acquisition and auditory-motor interactions. We hypothesise that a deficit in the pathways to the associative cortex is one of the neural factors in LKS.

\subsection{INTRODUCTION}

\section{Landau-Kleffner Syndrome}

Landau-Kleffner Syndrome (LKS) is a form of acquired aphasia in children, characterized by epilepsy and epileptiform discharges in the electroencephalogram (EEG) [1]. Initial speech and language development is normal in affected children, but at an age of 3 to 10 years, these children develop (semi-) acute aphasia in a period of days to months. Aphasia is mostly receptive (phonological decoding) and develops into expressive disorders at a later stage [2]. Auditory agnosia is also reported [3]. Patients may appear deaf/mute and often suffer from severe behavioural problems due to lack of means of communication [4].

During the active epileptic phase, the EEG is abnormal and rare nocturnal focal motor or secondarily generalized seizures or atypical absences may be observed. The awake EEG shows focal or multi-focal spikes-and-waves predominantly over temporal regions, while sleep EEG shows an activation of interictal EEG abnormalities and during the acute epileptic phase NREM (Non Rapid Eye Movement sleep) sub-continuous or continuous spikes-and-waves (CSWS) are observed [3].

In LKS patients, the audiogram is usually normal [5] and auditory information is processed normally up to the primary auditory cortex as demonstrated by EEG recordings [6]. However in LKS patients short term verbal memory is severely disrupted, even years after the active epileptic phase [7]. This specific 

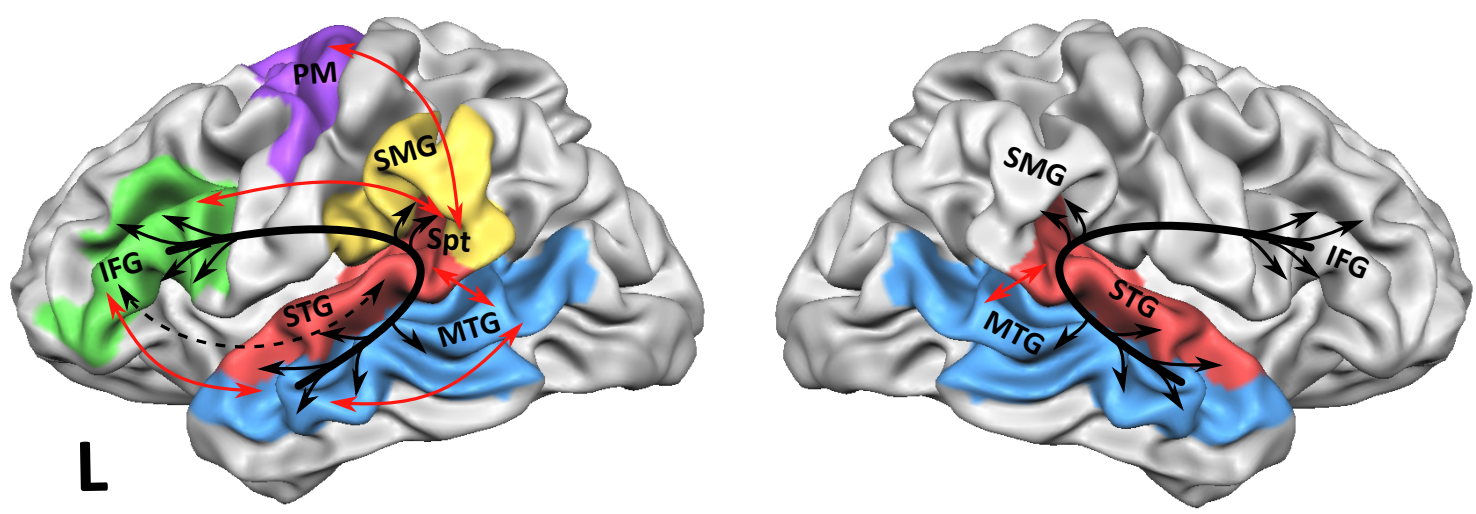

Figure 6.1: Functional anatomy of language processing (the dual stream model, see text) in the brain shown in red. Anatomical structures as found by DTI tractography are shown in black. The main connection is the Arcuate fasciculus (AF) connecting IFG with STG/MTG. Secondary pathways (dashed black line) may also exist but are hard to delineate with current DTI methods. IFG: Inferior Frontal Gyrus STG/MTG: Superior/Middle Temporal Gyrus SMG: Supramarginal Gyrus PM: premotor cortex Spt:Sylvian parietal temporal region.

impairment in short-term verbal memory is thought to arise from focal dysfunctions in the temporal cortex bilaterally [3].

To date, there is no anatomical evidence from structural MRI explaining the deficits in LKS. Structural MRI is usually normal [8, 9, 10]. In some rare, atypical cases of LKS specific pathologies such as cysticercosis or astrocytomas have been reported [9].

After reaching adolescence, the EEG typically returns to normal and the patient is considered to be recovered from LKS [11]. However, in most cases, severe speech and/or language deficits remain when the epileptic phase has terminated. Spontaneous recovery may occur but is unlikely. When treatment focussing on restoring communication is offered using visuo-motor therapy part of the language capabilities may be restored [12].

Most of the research conducted on LKS is done in a clinical context and uses EEG as its primary modality. A recent review of the EEG-associated literature in LKS is available in [11], where it is suggested that the cortex and the U-shaped fibers in the white matter are possible related to language deficits. In this paper, we will focus on detailed neuroanatomical white matter structures in a recovered LKS patient, and we will attempt to link neuroanatomy to language deficits in LKS. We will first review functional and white matter anatomy of language networks in the healthy human brain. 


\section{Functional Anatomy of Language and Language Working Memory}

The functional neuroanatomy of language in the healthy brain is becoming better and better understood in recent years. Starting from the signal in the primary auditory cortex, functional MRI data shows that speech sounds invokes activation in Heschl's gyrus (HG), areas in anterior superior temporal gyrus (STG) and superior temporal sulcus (STS), as well as regions in inferior frontal gyrus (IFG) [13], suggesting that HG is connected to the aforementioned regions.

The recent "dual stream model" [14] argues that early speech processing beyond the primary auditory cortex occurs bilaterally in the dorsal STG and STS, see figure 6.1 in red. Later processing is split up in two pathways: There is a bilateral ventral stream for speech comprehension and recognition, involving structures in superior and middle temporal lobe and a strongly left-dominant dorsal stream for sensory-motor integration or the translation of speech signals into articulatory representations in the frontal lobe [15]. The dorsal stream includes a sylvian parietal temporal region (Spt) at the parietal-temporal junction and areas in the posterior frontal lobe [16]. This area is densely connected to the pars opercularis as revealed by DTI-based fiber tracking [17].

Since LKS patients have severe dysfunction of verbal short-term memory, it is sensible to look into networks for working memory and language. These networks are anatomically partially overlapping [18]. Some areas in the language network (especially IFG) are thought to be involved in both language and working memory. The IFG is assumed to assemble strings of information into meaningful messages [18]. Area Spt, located closely to supramarginal gyrus -which is involved in general short term memory- is thought to link motor information from the vocal tract with auditory signals and thereby supporting verbal short-term memory [15].

\section{Anatomy of the Language Network}

White matter structure can be investigated non-invasively with DW-MRI. By probing for microscopic water diffusion in white matter axons with MRI and following the main direction of the diffusion tensor (diffusion-tensor based fiber tracking "DTI-based fiber tracking"), this technique allows for reconstruction of WM fiber tracts in the brain (for an overview of the technique, see e.g. [19]).

The main white matter language tracts (arcuate fasciculi AF, see figure 6.1) connecting IFG with the STG and MTG have been investigated to a large extend in healthy subjects with DW-MRI (e.g. [20, 21, 22, 23]). The AF is found in the left hemisphere and an analogue to the AF can also be found in the right hemisphere. Howerever, the AF is reported to be highly left-lateralized, that is, the number of reconstructed fiber tracts of the AF is larger in the left hemisphere than in the right hemisphere [21]. Not only the AF is connecting IFG with STG/MTG: 
Additional white matter pathways connecting IFG with STG/MTG in the left hemisphere have been reported. A dorsal pathway runs from IFG via the longitudinal superior fasciculus (SLF) to the posterior temporal lobe and STG/MTG. However, using current DTI tractography techniques this pathway is hard to separate from the AF [24]. A ventral pathway runs from IFG to the anterior STG via the ventral part of the extreme capsule and uncinate fasciculus. Again, this is a hard to delineate fiber tract with current DTI tractography techniques [24]. The dorsal and ventral streams might be functionally similar to the dual stream model described before, but is still under discussion.

The tracts originating from the primary auditory cortex (Heschl's Gyrus HG in particular) have not been studied extensively with DTI tractography. One study reports that HG is connected with one pathway to posterior STG and to anterior STG via a second pathway [23]. Another study reports more extensive pathways; left hemispheric pathways from HG connecting to IFG and insular cortex, while in the right hemisphere fiber tract reconstructions run from $\mathrm{HG}$ and terminate in parietal and occipital lobe and insular cortex [25].

\section{Goal of this Paper}

The neural mechanisms behind LKS and the anatomical deficit (if present) causing aphasia and agnosia are not yet understood. It is hypothesized that the deficit underlying LKS is generalized auditory amnesia and that therefore an impairment in the auditory circuit might exist [26]. But, to our knowledge, there are no studies investigating the white matter language pathways in LKS, partly due to lack of patient data and because LKS research is usually done in a clinical context where DW-MRI protocols are not always available.

In this paper, we investigate the auditory and language related white matter (WM) circuits, specifically the connections arising from the primary auditory cortex to investigate if HG is connected to higher language areas. We investigate the arcuate fasciculus to look into connections between IFG and superior STG and STS in a subject with LKS after the epileptic phase with DTI-based fiber tracking. We will describe the tracts and deviations from results found in healthy control subjects.

\subsection{METHODS}

\section{Case Description}

We present the case of a 27 year old female I., where the language capabilities have recovered after the epileptic phase in great extend due to intensive visuomotor communication therapy. 
I. was diagnosed LKS at 5 years old (a complete case history can be found in [27]). At that time, language production and language comprehension is severely disrupted. Her speech-language disorders seemed to be caused by sensory aphasia. Additionally, language perception was severely disrupted on a word/sentence level and she was diagnosed with auditory agnosia and jargonaphasia. She was diagnosed a mixed-type aphasia, which is not solemnly caused by auditory agnosia, combined with a very weak auditory memory. Expressive language was severely impaired as well as was the abstraction of inner language. Memory for rhythm was pathologically weak, as were fine motor skills and memory for order of motor tasks. No evidence for dyspraxia was reported, but there was a highly related pattern. Visual memory was strong, she had developed a coding strategy for communication. Lastly, non-verbal abstraction was weak and hence dys-symbolic problems were to be expected.

On the executive part of an intelligence test, she scored in the average to aboveaverage (IQ 115) at the age of seven. Performance on visual memory was equal or above average compared to her age group. In a non-verbal auditory discrimination task, five out of ten sounds were classified correctly. Spontaneous speech was characterised by jargon-aphasia. Some meaningful two- and multiple-word sentences are produced, at the articulation test, almost all words were correctly pronounced. Errors consisted of word reduction. However, she was severely impaired on auditory discrimination and, comprehension of sentence structure, fluency of speech and auditory memory.

Comprehension of spoken language was very limited and occurred only when enough cues were presented. Speech-reading was difficult. Her learning conditions for oral language, however, were so weak that communication by speech was not possible during this time. Simultaneous memory was strong and written language was predicted to play an important role in language development. Successive memory was weak causing problems when reading finger-spelled language. However, after an introduction period active use of finger-spelling was possible. Understanding spoken language was poor; almost no words were being recognized based on hearing only

I. was admitted to a school for the deaf where therapy was focussed on restoring communication using visuo-motor therapy and finger spelling. The therapy was highly successful and I. is currently (at the age of 27) able to communicate verbally while keeping eye contact. She continues to make mistakes when speaking in word form, sentence construction, as well as melody and accent. I. has difficulty acquiring new vocabulary if presented verbally. In a group, she is able to follow the conversation if each speaker is speaking in concession. In work-related meetings a writing interpreter is used, which enables I. to understand the Dutch language to the full. Reading and writing skills are at a normal level. She got a degree as a teaching assistant on Dutch MBO level (comparable 
US Associate Degree). Currently she lives a full life as a married women with a full-time job.

\section{DW-MRI acquisition}

DW-MRI data were acquired at the Maastricht Brain Imaging Center. The experimental procedure was in accordance with Maastricht University ethical guidelines and participation was voluntary. Written consent was obtained from the subject.

Data was acquired on a $3 \mathrm{~T}$ Siemens Allegra system (Siemens, Erlangen, Germany) using a birdcage single coil. A double-refocussed diffusion weighted SE-EPI sequence [28] was used to minimize the effects of eddy current distortions. $752 \mathrm{~mm}$ slices covering the whole brain were acquired with matrix size $128 \times 128$, field of view $256 \times 256$, resulting in $2 \times 2 \times 2 \mathrm{~mm}$ voxels. 72 isotropically distributed diffusion directions at a b-value of $1000 \mathrm{~mm}^{2} / \mathrm{s}$ were acquired, as well as 7 interleaved bo volumes. TR/TE $9400 / 83 \mathrm{~ms}$, acquisition time was $12 \mathrm{~min} 53 \mathrm{~s}$.

For anatomical reference, a TI weighted anatomical data set (ADNI-MPRAGE) was acquired in the same session, with FOV $256 \times 256$, 192 slices at a $1 \times 1 \times$ $1 \mathrm{~mm}$ voxel size.

\section{DW-MRI data processing}

The DWI data was checked for motion by an affine registration of the bo volumes. No significant motion was detected (the registration error was well below I voxel), so no motion correction was performed. The data was checked for eddy current distortions using a modified version of the ICC algorithm [29]. Very little distortion was found due to the double-refocussed SE-EPI sequence and therefore eddy current correction was omitted.

The DWI data was co-registered to the anatomical data set in BrainVoyagerQX 2.2 (Brain Innovation BV, Maastricht NL). The diffusion tensors were calculated in BrainVoyagerQX and exported to in-house developed $\mathrm{C} / \mathrm{C}++$ fiber tracking software. For fiber tracking, an FA threshold of 0.15 was set. The angular threshold was set at 30 degrees and the step size at $0.5 \mathrm{~mm}$. Reconstructed fibers were visualized in BrainVoyagerQX on the anatomical data set.

From the anatomical data, the gray matter cortical sheet was reconstructed in BrainVoyagerQX. On the cortical sheet, Heschls gyrus was marked as a ROI in accordance with the delineation in [30]. The ROI was then back-projected onto the DWI data set in order to create a 2-D ROI. The ROIs needed to reconstruct the arcuate fasciculus were created on axial slices of the DW-MRI images according to the ROI definitions in $[31,21,32]$. 


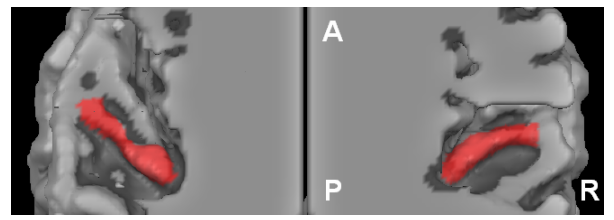

Figure 6.2: ROI definition of Heschl's gyrus on the cortical sheet.

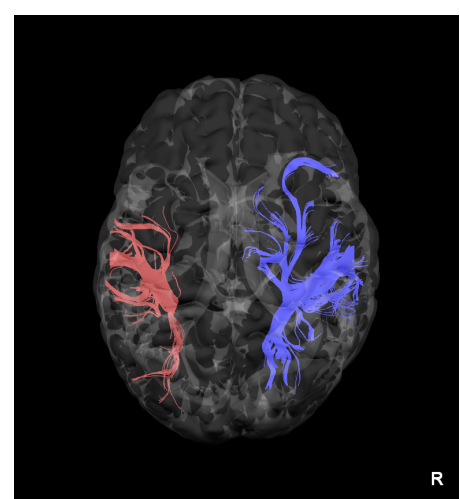

(a) HG fiber reconstructions seen from the top.

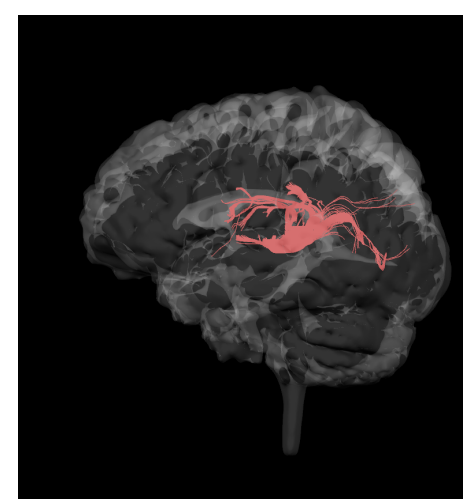

(b) HG fiber reconstructions in the left hemisphere.

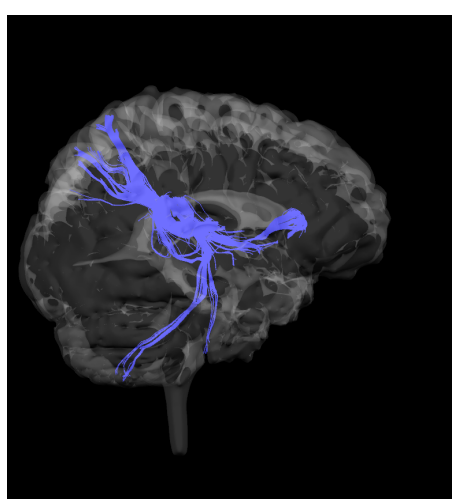

(c) HG fiber reconstructions in the right hemisphere.

Figure 6.3: Tractography reconstructions of pathways originating from Heschl's gyrus in both hemispheres. In both hemispheres, HG connects to the upper bank of the sylvian fissure. In the left hemisphere a segment projects into the occipital lobe. In the right hemisphere, HG tracts project to superior parietal cortex, a segment projects into Broca's area and a segment projects into the cerebellum via the amygdala.

For comparison of the reconstructed fiber tracts in this single case to control data, we used the arcuate fibers supplied in the white matter atlas from [33], which is composed of major fiber tracts in 40 right-handed healthy control subjects (20 male and 20 female) ageing 18-22 years. The anatomical image was co-registered to the average atlas brain anatomy using FSL-FLIRT [34].

The reconstructed AF fibers were back-projected on the subjects anatomical image, and then converted to Nifti using the Nifti-converter v1.08 for BrainVoyagerQX [35]. The registration matrix obtained from FSL-FLIRT was used to convert the reconstructed fibers of the arcuate fasciculus in I. into the atlas brain space. 


\subsection{RESULTS}

Fiber reconstructions from Heschl's gyrus

The reconstructed fiber tracts originating from left and right HG are shown in figure 6.3. From the left HG, see figure 6.3b, reconstructed fibers curve upwards around the sylvian fissure and project into the superior bank of the sylvian fissure and inferior frontal gyrus. A small portion of the fibers projects anteriorly into the tip of the temporal lobe, and posterior segment projects into the occipital lobe.

In the right hemisphere, see figure $6.3 \mathrm{c}$, a proportion of the reconstructed fibers curve around the posterior part of the sylvian fissure. A segment projects into superior parietal cortex, while another segment projects forwards medial to the sylvian fissure into the inferior frontal lobe. A third segment projects into the cerebellum.

\section{Arcuate fasciculus fiber reconstructions}

Fiber reconstructions of the arcuate fasciculus in both hemispheres are shown in figure 6.4. The left hemisphere AF reconstruction in figure 6.4a runs from the posterior parts of the superior and middle temporal gyri, then arches around the anterior part of the sylvian fissure and ends posterior of the inferior frontal gyrus.

The right hemisphere's AF main segment (blue in figure 6.4c) follows a similar path as in the left hemisphere, from the superior MTG/STG around the posterior part of the sylvian fissure to parts posterior of the inferior frontal gyrus. Two other segments were found in the tractography results; one posterior segment (green in figure 6.4c) running from superior MTG/STG to inferior parietal lobe, and a segment running from inferior parietal lobe towards parts posterior of the inferior frontal gyrus, which is the yellow segment in figure 6.4c.

In figure 6.5 the overlap of the left and right AF in I. with the WM atlas AF is shown. The reconstructed fibers in I. are in good agreement with the atlas. In the left hemisphere however, the segment connecting the temporal and inferior parietal lobe is not found in I., while it is present in the WM atlas of control subjects. In the right hemisphere, AF reconstruction is similar to the controls and we did find a segment connecting inferior parietal lobe with STG/MTG.

\subsection{DISCUSSION}

To the best of our knowledge, this is the first study to present white matter language pathways in a recovered LKS patient. LKS is a rare form of acquired aphasia in children, characterized by epilepsy and epileptiform discharges in the 


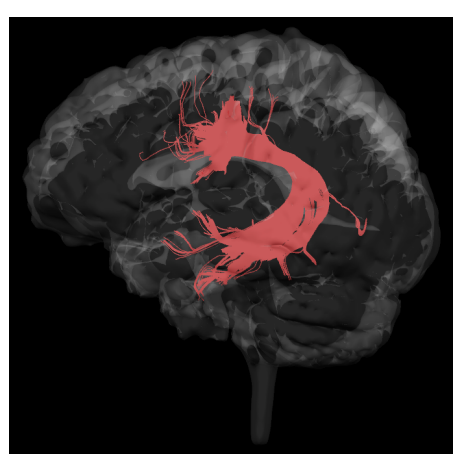

(a) Left $\mathrm{AF}$

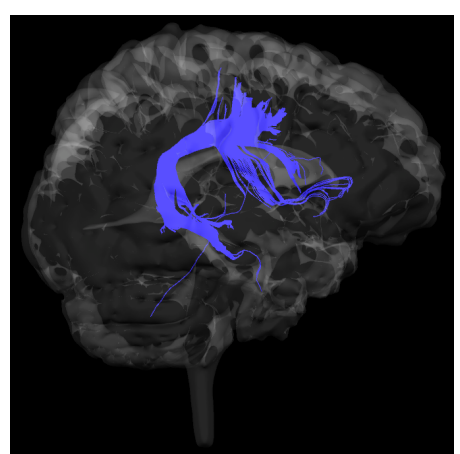

(b) Right AF

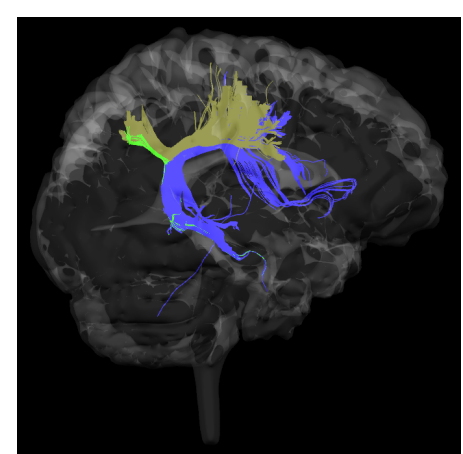

(c) Right AF segments.

Figure 6.4: Left and right arcuate fasciculus reconstruction.

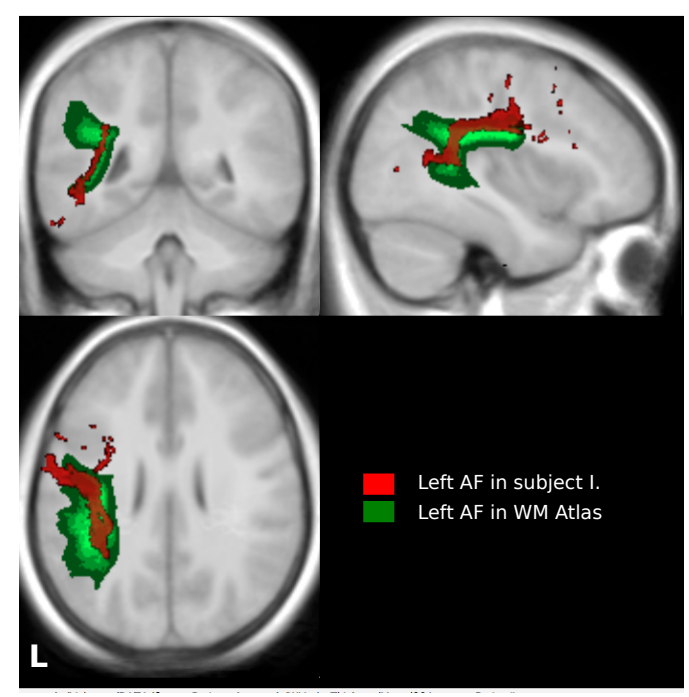

(a) Left AF in subject I. overlayed on the WM atlas.

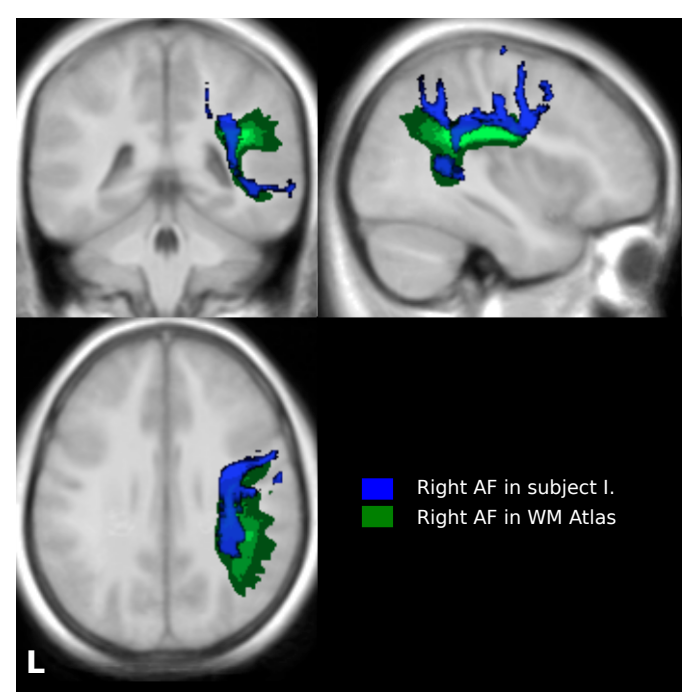

(b) Right AF in subject I. overlayed on the WM atlas.

Figure 6.5: The reconstructed AF fibers (red in the left hemisphere, blue in the right) in I. are in good agreement with the AF from the atlas (in green). In the right hemisphere we found a projection into the inferior parietal lobe, which is present in the atlas in both hemispheres, but was not found in the left hemisphere of I. 
electroencephalogram (EEG), resulting in severe language deficits. Language outcome of the syndrome, after the EEG has returned to normal when the patient reaches adulthood, is usually poor, but extensive visuo-motor therapy increases restoration of language capabilities. The neural mechanisms behind LKS are not yet understood and anatomical evidence for the language deficits have not been found with structural MRI. In this study, we investigated the white matter pathways originating from Heschl's gyrus as well as the main white matter structure in language, the arcuate fasciculus, with DTI-based fiber tracking. Summarizing the first finding in the current study, fibers originating from Heschl's gyrus seem to be left-right inverted with respect to HG fibers found in a control study [25]. As a second finding, the long segment of the arcuate fasciculus was found in both hemispheres. We did not find the short segments in the left arcuate fibers reported in healthy subjects [21,33], but we did find short arcuate segments in the right hemisphere.

Data on the white matter pathways originating from HG investigated with DW-MRI is sparse. One study found a posterior pathway between posterior regions and Heschls gyrus (HG) and posterior Superior Temporal Gyrus (STG) using DTI based probabilistic fiber tracking [36]. An anterior pathway, connecting anterior HG and anterior STG, was also found, but this pathway is less dense than the anterior pathway [36]. Another study using DTI based deterministic fiber tracking reports asymmetrical pathways [25] in 30 healthy subjects; in the left hemisphere reconstructed fibers pass beneath the planum temporale terminate in the insular cortex and inferior frontal gyrus. In the right hemisphere, fibers pass beneath planum temporale and terminate in parietal and occipital lobes. Evidence from functional MRI shows that HG is connected to anterior STG, STS and IFG in the left hemisphere [13], which seem to complement the white matter connection pattern.

In our results portions of the fibers originating from HG are projecting into anterior parts of the STG in both hemispheres, as well as additional fibers connecting HG in the left hemisphere with the pole of the temporal lobe and the occipital lobe. In the right hemisphere, we found additional fibers projecting towards inferior parietal lobe and inferior frontal lobe, which seem to be integrated with the arcuate fasciculus to some degree. Fibers descending into the cerebellum which might be part of the auditory nerve were also found. Interestingly, in I., the fiber tracts originating from right $\mathrm{HG}$ projecting to inferior frontal areas seem to resemble the left inferior frontal fibers found in healthy controls [25].

If we look at the arcuate fasciculus, it was shown in a study with 40 healthy subjects [21], in all subjects that STG/STS is connected to IFG via the long segment of the arcuate fasciculus (AF) in the left hemisphere. In the right hemisphere, the majority $(62.5 \%)$ of subjects did not have a homologue AF connection. In $20 \%$ of the subjects, the right AF was found to be weaker, and in $17.5 \%$ of subjects equal to the left AF. This indicates a leftward lateralization of the arcuate 
fasciculus. However, another study [31] reports the presence of a right AF homologue in $12 / 12$ subjects, but does not report a lateralization index, since only the right AF was investigated. A very recent study in 40 healthy subjects [33] reports again a strong leftward lateralization of the AF long segment. Our results show the presence of the long segment of the AF in both hemispheres. Additional to the long segment of the AF connecting superior STS and STG to IFG in the left hemisphere, there are two shorter segments reported to connect superior STS and STG to the inferior parietal lobule (associative auditory cortex) and connecting the inferior parietal lobule to IFG $[21,33]$. The shorter segments were also found in the right hemisphere $[21,31,33]$. We did not find evidence for these short segments of AF in the left hemisphere, while these segments are consistently found in the left hemisphere of healthy subjects. In the current study, the short segments projecting to the inferior parietal lobe were found to exist only in the right hemisphere.

These short segments in the left hemisphere are possible connecting the arcuate fasciculus with a section on the border of the left inferior parietal lobe and sylvian fissure, termed the sylvian-parietal temporal area (Spt) [14, 15], which is regarded to be crucial as a sensory-motor circuit for integration of speech or nonspeech sounds (e.g. music) and motor interaction. This circuit is also involved in phonological short-term memory. Consequently, this area is essential when learning to speak, online guidance of speech sequences, and for acquiring new vocabulary [14].

LKS patients in the active and recovered phase suffer from permanent dysfunction of associative auditory cortex as measured in a dichotic listening task during EEG recordings [37, 38, 39]. Furthermore, short-term phonological memory is consistently found to be impaired in LKS, even in patients who have recovered fairly well [7]. Short term phonological memory was found to be weak in I. in the early active stage of LKS [27]. Combined with the fact that I. currently continues to make mistakes in sentence construction, word form, melody and accent and has troubles with learning new vocabulary when presented verbally (observations by WP) might indicate that there is a dysfunction in online feedback of speech and short term phonological memory. Although speculative, the absence in I. of the short segments of the AF connecting AF with left inferior parietal and sylvian areas is a possible neuro-anatomical explanation for the observed language deficits.

The current study is limited in the sense that fiber tracts from an individual subject are investigated. To our knowledge, post-mortem data from LKS patients is not available and clearly we cannot resort to animal studies. Therefore, we need to rely on white matter atlases obtained in healthy subjects. A dissimilarity in fiber tracts found here and the atlas might also arise from differences in the regions of interest used to seed the fibers. The data in the atlas we used [33] was 
obtained on a $1.5 \mathrm{~T}$ system with a $b$-value of $1300 \mathrm{~s} / \mathrm{mm}^{2}$, and was processed with different software, which might influence the comparison.

The results presented here in fibers originating from Heschl's gyrus and the arcuate fasciculus suggest a rightward lateralisation of language-related WM pathways. Currently we can only speculate about the re-organization of these fibers in the right hemisphere. There is some evidence that in deaf signers, the right hemisphere is more involved in sign language processing than the left hemisphere [40], but this is being debated, see [41] for an overview. Since I's "native language" is sign language, the right hemisphere has possibly taken over language processing functions from the left hemisphere. A second possibility is that the left temporal lobe was more severely affected during the epileptic phase, causing the right hemisphere to take over some functions.

We hypothesise that the absence of arcuate fibers directed towards the left inferior parietal lobe as is the case in I. causes auditory information not to interact with the motor system, thereby restricting phonological short-term memory, (speech) sound-motor interaction and online feedback of speech and might be one of the neuronal factors underlying LKS.

Future research into the mechanisms underlying LKS should involve studies on lateralization of language tracts in multiple patients with DW-MRI and future functional MRI studies should be focussed on auditory sensory-motor interactions. Ideally, a long-term follow up study should be conducted on multiple patients to be able to follow white matter changes over time.

\section{REFERENCES}

[1] WM Landau and FR Kleffner. Syndrome of acquired aphasia with convulsive disorder in children. Neurology, 7:523-530, 1957.

[2] G Lanzi, P Veggiotti, S Conte, E Partesana, and C Resi. A Correlated Fluctuation of Language and EEG Abnormalities in a Case of the Landau-Kleffner Syndrome. Brain E Development, 16(4):329-334, 1994.

[3] E Hirsch, M Paola, G Rudolf, C Seegmuller, A De Saint, P Maquet, N Wioland, C Marescaux, and A Arzimanoglou. Landau - Kleffner syndrome is not an eponymic badge of ignorance. Epilepsy Research, pages 239-247, 2006.

[4] A Zivi, G Broussaud, S Daymas, J Hazard, and C Sicard. Epilepsia-acquired aphasia syndrome with psychosis - report of a case. Annales De Pediatrie, 37(6):391-394, 1990.

[5] CJ Feekery, B Parryfielder, and IJ Hopkins. Landau-Kleffner Syndrome 6 Patients Including Discordant Monozygotic Twins. Pediatric Neurology, 9(1):49-53, 1993 . 
[6] OK Steinlein. Epilepsy - aphasia syndromes. Expert Rev Neurother, 9(6):825833, 2009.

[7] S Majerus, S Laureys, F Collette, G Del Fiore, C Degueldre, A Luxen, M Van Der Linden, P Maquet, and MN Metz-Lutz. Phonological short-term memory networks following recovery from Landau and Kleffner syndrome. Hum. Brain Mapp., 19:133-144, 2003.

[8] M Bureau. Continuous spikes and waves during slow sleep (CSWSS): Definition of a syndrome. In M.B.A. Beaumanoir, T. Deonna, L. Mira, and C. A. Tassinari, editors, Continuous spikes and waves during slow sleep, pages 17-26. Libbey, London, UK, 1995.

[9] M Van Hirtum-Das, EA Licht, S Koh, JY Wu, WD Shields, and R Sankar. Children with ESES: Variability in the syndrome. Epilepsy Research, 70:S248S258, 2006.

[10] M Fandiño, M Connolly, L Usher, S Palm, and FK Kozak. Landau-Kleffner syndrome: A rare Auditory Processing Disorder Series of cases and review of the literature. Int J Pediatric Otorhinolaryngology, 75:33-38, 2011.

[11] GM Overvliet, RMH Besseling, JSH Vles, PAM Hofman, WH Backes, MHJA van Hall, S Klinkenberg, J Hendriksen, and AP Aldenkamp. Nocturnal epileptiform EEG discharges, nocturnal epileptic seizures, and language

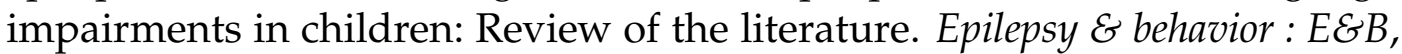
19:550-558, 2010.

[12] T Deonna, AC Prelaz-Girod, C Mayor-Dubois, and E Roulet-Perez. Sign language in Landau-Kleffner syndrome. Epilepsia, 50(suppl 7):77-82, 2009.

[13] SK Scott and IS Johnsrude. The neuroanatomical and functional organization of speech perception. Trends in Neurosciences, 26:100-107, 2003.

[14] G Hickok and D Poeppel. The cortical organization of speech processing. Nature Reviews Neuroscience, 8:393-402, 2007.

[15] G Hickok. The functional neuroanatomy of language. Physics of life reviews, 6(3):121-43, September 2009.

[16] G Hickok, J Houde, and F Rong. Sensorimotor Integration in Speech Processing: Computational Basis and Neural Organization. Neuron, 69:407-422, 2011.

[17] BR Buchsbaum, J Baldo, K Okada, KF Berman, N Dronkers, M D'Esposito, and G Hickok. Conduction aphasia, sensory-motor integration, and phonological short-term memory - An aggregate analysis of lesion and fMRI data. Brain and language, pages 1-10, January 2011. 
[18] I Timmers, J van den Hurk, F Di Salle, ME Rubio-Gozalbo, and BM Jansma. Language production and working memory in classic galactosemia from a cognitive neuroscience perspective: future research directions. Journal of inherited metabolic disease, 2011.

[19] S Mori, W E Kaufmann, C Davatzikos, B Stieltjes, L Amodei, K Fredericksen, G D Pearlson, E R Melhem, M Solaiyappan, and G V Raymond. b. Imaging cortical association tracts in the human brain using diffusion-tensor-based axonal tracking. Magn. Reson. Med., 47:215-223, 2002.

[20] M Catani, DK Jones, and DH fFytche. Perisylvian language networks of the human brain. Annals of neurology, 57(1):8-16, January 2005.

[21] M Catani, MPG Allin, M Husain, L Pugliese, MM Mesulam, RM Murray, and DK Jones. Symmetries in human brain language pathways correlate with verbal recall. PNAS, 43(23):17163-17168, 2007.

[22] S Frey, JSW. Campbell, GB Pike, and M Petrides. Dissociating the human language pathways with high angular resolution diffusion fiber tractography. Journal of Neuroscience, 28(45):11435-11444, 2008.

[23] MF Glasser and JK Rilling. DTI Tractography of the Human Brain's Language Pathways. Cerebral Cortex, 18:2471-2482, 2008.

[24] AD Friederici. Pathways to language : fiber tracts in the human brain. Trends in Cognitive Sciences, 13:175-181, 2009.

[25] TR Barrick, IN Lawes, and CA Clark. White Matter Pathway Asymmetry Corresponds to Auditory Spatial and Language Lateralisation. Proc ISMRM, 11:334, 2004.

[26] VM Pedro and G Leisman. Hemispheric integrative therapy in LandauKleffner Syndrome: Applications for rehabilitation sciences. International Journal of Neuroscience, 115(8):1227-1238, 2005.

[27] WJLM Pullens. [Landau-Kleffner Syndrome; the effect of fingerspelling on language processing] (in Dutch). Master's thesis, University of Tilburg, The Netherlands, 1995.

[28] TG Reese, O Heid, RM Weisskoff, and VJ Wedeen. Reduction of EddyCurrent-Induced Distortion in Diffusion MRI Using a Twice-Refocused Spin Echo. Magnetic Resonance in Medicine, 182:177-182, 2003.

[29] J Zhuang, J Hrabe, A Kangarlu, D Xu, R Bansal, CA Branch, and BS Peterson. Correction of Eddy-Current Distortions in Diffusion Tensor Images Using the Known Directions and Strengths of Diffusion Gradients. Journal of Magnetic Resonance Imaging, 1193:1188 -1193, 2006. 
[3o] IS Sigalovsky, B Fischl, and JR Melcher. Mapping an intrinsic MR property of gray matter in auditory cortex of living humans: a possible marker for primary cortex and hemispheric differences. NeuroImage, 32:1524-37, 2006.

[31] A Gharabaghi, F Kunath, M Erb, R Saur, S Heckl, M Tatagiba, W Grodd, and H-O Karnath. Perisylvian white matter connectivity in the human right hemisphere. BMC Neuroscience, 6:10-15, 2009.

[32] M Catani and M Mesulam. The arcuate fasciculus and the disconnection theme in language and aphasia : History and current state. Neurology, 44:953-961, 2008.

[33] M Thiebaut de Schotten, DH Ffytche, A Bizzi, F Dell'Acqua, M Allin, M Walshe, R Murray, SC Williams, DGM Murphy, and M Catani. Atlasing location, asymmetry and inter-subject variability of white matter tracts in the human brain with MR diffusion tractography. NeuroImage, 54:49-59, 2011.

[34] SM Smith et al. Advances in Functional and Structural MR Image Analysis and Implementation as FSL. NeuroImage, 23(S1):208-219, 2004.

[35] H Breman and R. Goebel. Nifti converter plugin manual for Brainvoyager QX. Brain Innovation BV, 2010.

[36] J Upadhyay, A Silver, T Knaus, K Lindgren, M Ducros, D-S Kim, and $\mathrm{H}$ Tager-Flusberg. Effective and structural connectivity in the human auditory cortex. The Journal of neuroscience, 28:3341-9, 2008.

[37] N Wioland, G Rudolf, and MN Metz-lutz. Electrophysiological evidence of persisting unilateral auditory cortex dysfunction in the late outcome of Landau and Kleffner syndrome. Clin. Neurophysiol., 112:319-323, 2001.

[38] M Plaza, MT Rigoard, C Chevrie-Muller, H Cohen, and A Picard. Shortterm memory impairment and unilateral dichotic listening extinction in a child with Landau-Kleffner syndrome: Auditory or phonological disorder? Brain and Cognition, 46(1-2):235-240, 2001.

[39] MN Metz-Lutz, A de Saint Martin, E Hirsch, P Maquet, and C Marescaux. Impairment in auditory verbal processing and dichotic listening after recovery of epilepsy in Landau and Kleffner syndrome. Brain and Cognition, 40(1):193-197, 1999.

[40] HJ Neville, D Bavelier, D Corina, J Rauschecker, A Karni, A Lalwani, A Braun, V Clark, P Jezzard, and R Turner. Cerebral organization for language in deaf and hearing subjects: biological constraints and effects of experience. Proceedings of the National Academy of Sciences of the United States of America, 95:922-9, 1998. 
[41] M MacSweeney, CM Capek, R Campbell, and B Woll. The signing brain: the neurobiology of sign language. Trends in Cognitive Sciences, 12:432-440, 2008. 

this Chapter is BASEd on Marco Tamietto*, Pim Pullens*, Lawrence Weiskrantz, Rainer Goebel and Beatrice de Gelder. Subcortical anatomical connectivity to human amygdala and its changes following early visual cortex lesion, Current Biology, accepted.

*both authors contributed equally to this paper. 


\section{ABSTRACT}

There is extensive evidence that emotional signals can be processed without being consciously perceived. neuroimaging studies have demonstrated that the subcortical structures amygdala, superior colliculus, basal ganglia and pulvinar are activated when non-conscious emotional stimuli are perceived. Moreover, when these emotional stimuli are perceived consciously, they elicit a similar or even decreased response compared to the non-conscious responses.

Investigations into the neural underpinnings of non-consciously perceived emotional stimuli are hard to perform on healthy subjects, since the application of the non-consciously 'visible' stimuli makes them spatially and temporally different from the consciously visible stimulus.

To investigate this phenomenon we can however revert to patients suffering from affective blindsight. These patients with cortical blindness following destruction of the primary visual cortex are nevertheless able to make remarkably accurate judgements about the emotional expression of stimuli projected in the portion of the visual field region affected by the lesion (scotoma).

We have investigated the subcortical pathways between superior colliculus, pulvinar and amygdala in five healthy matched controls and in blindsight patient GY. We have found evidence for a superior colliculus-pulvinar-amygdala pathway bilaterally in GY. This pathway was not present in the left hemisphere of the controls, while a right hemisphere pathway was found in 2/5 subjects.

\subsection{INTRODUCTION}

There is extensive evidence that emotional signals can be processed without being consciously perceived [1]. Visual stimuli with emotional significance that are non-consciously perceived still evoke responses in subcortical nuclei [2]. Using backward-masking e.g. [3, 4] or binocular rivalry $[5,6,7]$, neuroimaging studies have demonstrated that the subcortical structures amygdala, superior colliculus, basal ganglia and pulvinar are activated when non-conscious emotional stimuli are perceived. Moreover, when these emotional stimuli are perceived consciously, they elicit a similar or even decreased response compared to the non-conscious responses.

Investigations into the neural underpinnings of non-consciously perceived emotional stimuli are hard to perform on healthy subjects, since the application of the non-consciously 'visible' stimuli makes them spatially and temporally different from the consciously visible stimulus.

To investigate this phenomenon we can however revert to patients suffering from affective blindsight. These patients with cortical blindness following destruction of the primary visual cortex are nevertheless able to make remarkably 
accurate judgements about the emotional expression of stimuli projected in the portion of the visual field region affected by the lesion (scotoma).

Investigations of the neural underpinnings of affective blindsight have so far used functional MRI methods and revealed a functional connectivity between superior colliculus, pulvinar and amygdala as shown in the overview of visual and emotional pathways in figure 7.1. It was shown that this functional network is positively activated in response to non-consciously perceived fearful facial expressions in both patients with lesions in the extrastriate cortex [3] using PET as in healthy subjects [4] using fMRI based functional connectivity analysis. These results suggest the existence of totally subcortical $\mathrm{V}_{1}$-independent pathway to process visual emotional signals. We have investigated the subcortical pathways between superior colliculus, pulvinar and amygdala and have found bilaterally evidence for such a pathway in blindsight patient GY. This putative subcortical pathway is strikingly parallel with analogous evidence in animal studies indicating direct anatomical connections between the superior colliculus and the pulvinar and between the pulvinar and the amygdala that bypass $\mathrm{V}_{1}$.

A subcortical pathway exists in birds and rats for fast processing of coarse emotional stimuli. In birds, the analogue of the superior colliculus (the optic tectum), is directly connected to the analogue of the pulvinar (the nucleus rotundus) and both relay information to the amygdala analogue (the taenia) [8].

In rats visual information is transferred to the amygdala through direct anatomical connections from the superior colliculus to the lateral nucleus of the amygdala via the thalamus [9].

In non-human primates the presence of these direct anatomical connections has not yet been demonstrated. A connection between superior colliculus and inferior parts of the pulvinar and a connection from the medial pulvinar to the amygdala have been shown to exist but evidence for direct connections from inferior to medial parts of the pulvinar has yet not been established.

In the studies performed on humans with fMRI, covariation of functional responses in neuroimaging data does not indicate direct anatomical connectivity amongst the implicated regions. So, the existence of anatomical colliculuspulvinar-amygdala connections in the human brain is still unknown.

Moreover, it is unclear whether the ability to process emotional signals in the absence of $V_{1}$ results from the recruitment and strengthening of pathways existing also in the healthy brain, as fMRI data on non-conscious processing of emotions in neurologically intact observers seem to suggest, or are due to massive post-lesion neuronal reorganization that induce the formation of new pathways not otherwise present.

To study anatomical connectivity non-invasively in the brain, we can use diffusion-weighted magnetic resonance imaging (DW-MRI). DW-MRI is sensitive to diffusion of water molecules and it allows for quantification of the amount and directionality of water diffusion using the diffusion tensor [10] and can 

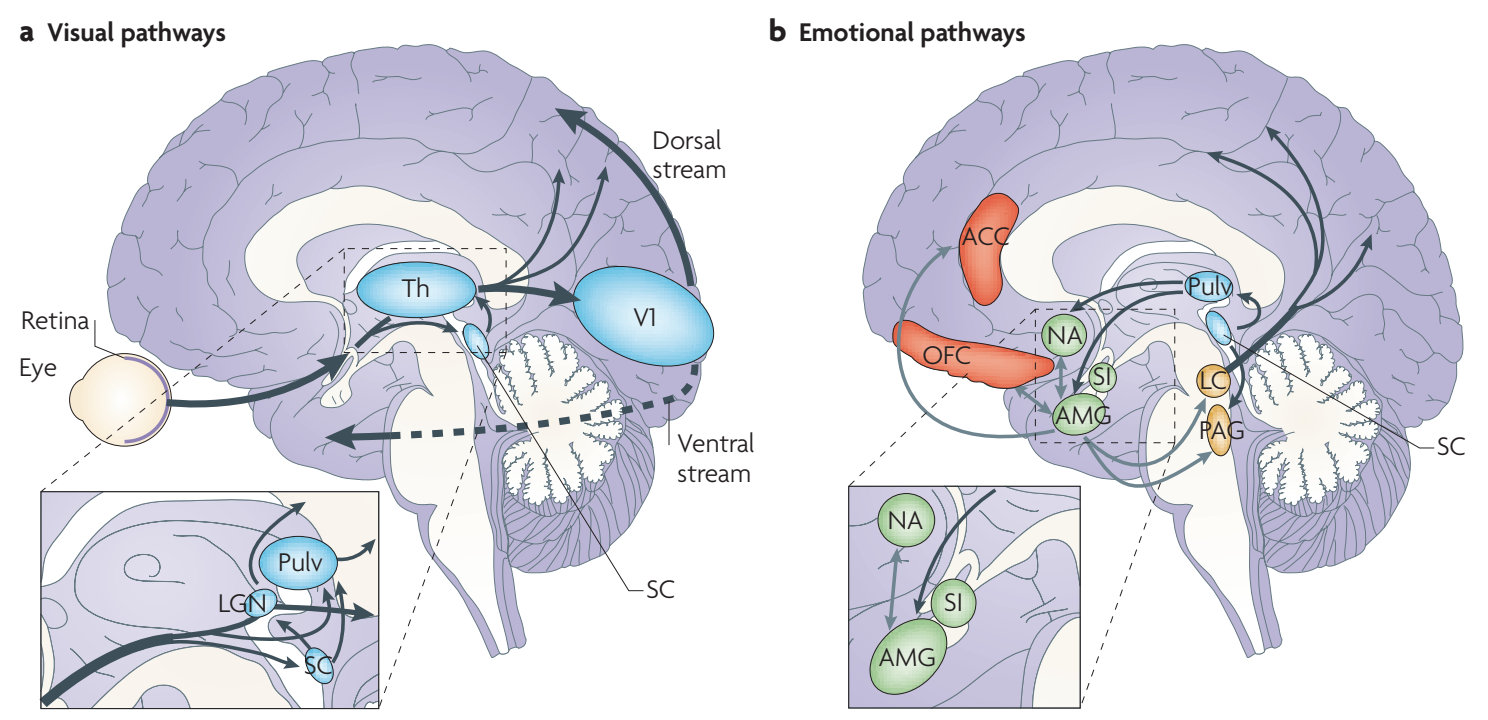

Figure 7.1: a The primary visual pathway (shown by thick arrows) originates from the retina and projects to the primary visual cortex (V1) in the occipital lobe via an intermediate station in the lateral geniculate nucleus (LGN) of the thalamus (Th). From V1, visual information reaches the extrastriate cortex along the ventral (occipitotemporal) and the dorsal (occipitoparietal) stream. However, a minority of fibres originating from the retina take a secondary route (shown by thin arrows) and reach both the superior colliculus (SC) and the pulvinar (Pulv). These two subcortical sites are connected and also send direct projections to the extrastriate visual cortex, bypassing V1. Another V1-independent visual pathway consists of the direct projections between the superior colliculus and the LGN that, in turn, send efferents to extrastriate cortices in the dorsal stream. $\mathbf{b}$ The 'emotion system' includes several cortical and subcortical areas. Among the subcortical structures are the amygdala (AMG) and the substantia innominata (SI; shown in green), which are buried deeply in the temporal lobe and in the basal forebrain, respectively, the nucleus accumbens (NA) in the basal ganglia (shown in green) and brainstem nuclei (shown in yellow), such as the periaqueductal grey (PAG) and the locus coeruleus (LC). Among cortical areas (shown in red) are the orbitofrontal (OFC) and the anterior cingulate cortex (ACC). The visual and emotional systems are extensively interconnected, especially at the subcortical level, where the superior colliculus is connected to the amygdala via the pulvinar. Direct connections also exist between subcortical and cortical emotion regions (for example, between the amygdala and OFC or ACC), between subcortical structures for emotions and cortical visual areas (for example, between the amygdala and temporal cortex) (not shown) and between brainstem nuclei and the cortex via diffuse projections (shown only from the LC). Grey arrows indicate connections within the emotion system. Reprinted by permission from Macmillan Publishers Ltd: Nature Neuroscience 11:697-709, (C) (2010). 
therefore probe for brain tissue microstructure. By following the main diffusion direction in the tissue, diffusion tensor imaging (DTI) based tractography enables us to make in-vivo virtual dissections of white matter structures in the brain non-invasively e.g. [11, 12].

In the present study we have used DTI based tractography to investigate direct anatomical connections between anatomically defined superior colliculus, pulvinar and amygdala in affective blindsight patient GY and 5 age-matched neurologically intact controls.

\subsection{METHODS}

\section{Subjects}

Five age (age 48, 49, 53, 6o and 62 years) and gender matched male control subjects who had no history of neurological disorders were asked to participate in the study. The subjects agreed on participation in accordance to the ethical guidelines set by Maastricht University and gave informed consent in accordance with the Declaration of Helsinki. GY's age at time of scanning was 50 years.

\section{Data acquisition}

Data for all subjects were acquired at Maastricht University, NL on a $3 \mathrm{~T}$ Siemens Allegra system (Siemens, Erlangen, Germany). Diffusion Weighted MRI data was acquired, using a single-channel birdcage coil, with a double-refocused echoplanar imaging sequence [13] to reduce the effect of eddy current distortions. In each subject, $752 \mathrm{~mm}$ thick axial slices with a matrix size of $128 \times 128$ and a Field-of-View of $256 \times 256 \mathrm{~mm}$ were acquired, resulting in $2 \times 2 \times 2 \mathrm{~mm}$ isotropic voxels. At a b-value of $1000 \mathrm{~s} / \mathrm{mm}^{2}, 48$ isotropically distributed diffusion gradient directions [14] were acquired. The diffusion scans were interleaved with $6 \mathrm{~b}=\mathrm{o}$ scans. Acquisition time for the diffusion scan was $8 \mathrm{~m} 27 \mathrm{~s}$. In the same session, a $1 \times 1 \times 1 \mathrm{~mm}$ T1 weighted ADNI-MPRAGE anatomical scan was performed.

\section{Diffusion data analysis}

Data was analysed in BrainVoyagerQX 2.1 (Brain Innovation, Maastricht, NL). The diffusion data were co-registered to the anatomical data using a normalized gradient fields approach [15]. The diffusion tensor [10] was calculated in each voxel. The data was exported to an in-house developed $\mathrm{C} / \mathrm{C}++$ tool for fiber tracking. Streamline fiber tracking using the local tensor as a projection operator [16] was performed. The data for each subject was checked for motion and 
eddy current artefacts. Both motion (see table 7.1) and eddy current distortions were well below $1 \mathrm{~mm}$, so no correction was applied.

Table 7.1: Mean $(\bar{x})$ and standard deviation (SD) of motion parameters found in all subjects. Translations $\mathrm{dx}, \mathrm{dy} \mathrm{dz}$, in [mm], rotations $\mathrm{rx}, \mathrm{ry}, \mathrm{rz}$ in [degrees]

\begin{tabular}{lllccccc} 
Subject & & $\mathbf{d x}$ & $\mathbf{d y}$ & $\mathbf{d z}$ & $\mathbf{r x}$ & $\mathbf{r y}$ & $\mathbf{r z}$ \\
\hline FK & $\bar{x}$ & 0.00090 & 0.00130 & 0.00210 & -0.00020 & 0.00100 & -0.00350 \\
& SD & 0.00060 & 0.00080 & 0.00120 & 0.00010 & 0.00060 & 0.00230 \\
FS & $\bar{x}$ & 0.00040 & 0.00080 & 0.00200 & -0.00020 & -0.00060 & 0.00140 \\
& SD & 0.00020 & 0.00060 & 0.00130 & 0.00010 & 0.00030 & 0.00090 \\
GL & $\bar{x}$ & 0.01280 & -0.01600 & 0.00890 & -0.00060 & -0.00090 & 0.00050 \\
& SD & 0.00010 & 0.00120 & 0.00310 & 0.00040 & 0.00060 & 0.00030 \\
PB & $\bar{x}$ & 0.00110 & 0.00170 & 0.00120 & 0.00010 & 0.00030 & -0.00200 \\
& SD & 0.00060 & 0.00080 & 0.00060 & 0.00010 & 0.00020 & 0.00110 \\
TH & $\bar{x}$ & 0.00003 & 0.00050 & 0.00054 & -0.00010 & 0.00016 & -0.00018 \\
& SD & 0.00001 & 0.00027 & 0.00029 & 0.00005 & 0.00011 & 0.00009 \\
GY & $\bar{x}$ & 0.00005 & 0.00184 & 0.00152 & -0.00017 & -0.00066 & 0.00007 \\
& SD & 0.00003 & 0.00135 & 0.00091 & 0.00009 & 0.00035 & 0.00009
\end{tabular}

Seed regions (ROIs) were defined by a skilled neuro-anatomist (MT) on the native space anatomical data. The seed regions included left and right amygdala (AMG), pulvinar (PLV) and superior colliculus (SC). The diffusion weighted MRI data was co-registered to the anatomical data. The ROIs were re-sampled to match DW-MRI data resolution. Per voxel, $7 \times 7 \times 7$ seed points were defined. The stopping threshold for fiber tracking was set at $F A=0.15$. Secondary ROIs were used to constrain the streamlines from the fiber tracking (AND operation). Fiber tracking was performed backward and forward between AMG-PLV, AMGSC and PLV-SC and SC-PLV-AMG. Unless noted otherwise, reported number of fibers are the sum of forward and backward reconstructed fibers.

A measure of connectivity $C_{A \rightarrow B}$ as proposed by [17] was calculated to quantify connection strength. This measure takes the size of the region of interest into account, and therefore provides a normalized connectivity measure. The measure reflects the "mean neuronal activity" $N_{A}$ of a region $A$ with size $R_{A}$, over the "mean neuronal activity" $N_{B}$ of another region $B$ with size $R_{B}$ and is defined as

$$
C_{A \rightarrow B}=\frac{N_{B}}{N_{A}} \propto \frac{T_{B}}{T_{A}} \cdot \frac{R_{A}}{R_{B}},
$$

where $T_{A}$ is the number of reconstructed tracts from region $A$ alone, and $T_{B}$ the number of reconstructed tracts originating from $A$ and reaching region $B$. For 
each set of ROIs $(A, B)$, the total connectivity $\left(C_{A \rightarrow B}+C_{B \rightarrow A}\right)$ was computed. For calculation of the connection strength between two regions $(A, B)$ via a third region $Q$, equation 7.1 was adapted as follows:

$$
C_{A \rightarrow B \text { via } Q}=\frac{T_{Q B}}{T_{A}}\left(\frac{R_{A}}{R_{Q}}+\frac{R_{Q}}{R_{B}}\right),
$$

with $T_{Q B}$ the number of fibers from $A$ reaching $B$ via $Q$ and $R_{Q}$ the size of region $Q$. This connectivity was calculated for each combination of ROIs, and then added up to get to a connectivity value for the three regions combined.

The connectivities proved to be normally distributed across subjects, as assessed by a Lilliefors test ("lillietest" function in Matlab) and therefore a onesample $t$-test was used for statistical evaluation of connection strength.

To enable cross-subject reconstructed fiber tract comparison, the anatomical data of each subject was transformed into Talairach (TAL) space [18] and the same TAL transformation was applied to the back-projected fibers in anatomical space. The resulting reconstructed fiber tracts in TAL space were back-projected into 2-D space to create a probabilistic map of the overlapping fibers.

\section{$7 \cdot 3$ RESULTS}

\section{Seed regions}

DTI based fiber tracking was initiated from voxels in the anatomically defined left and right amygdala, pulvinar and superior colliculus ROIs in GY, as shown in figure 7.2. ROI definitions in GY closely match the definitions in the controls. Similar ROIs were defined in all five controls. These ROIs to a high degree as is shown in figure 7.2 for the Superior Colliculus, Pulvinar and Amygdala ROIs in the control subjects.

The volumes of the seed regions, see Table $7 \cdot 2$, are quite similar across all subjects. The absolute number of fibers found from connection in each subject can be found in table $7 \cdot 3$.

\section{Superior colliculus - pulvinar - amygdala connections}

We reconstructed the fiber tracts between SC-PLV-AMG in both hemispheres using a combined AND operation: (SC \& PLV) \& AMG, (PLV \& SC) \& AMG, and finally (AMG \& PLV) \& SC. The other combinations of the three ROIs yielded exactly the same results and were therefore omitted from the procedures.

Figure 7.3A shows the reconstructed fiber tracts in GY . Fiber tracts in the left hemisphere are shown in light green, right hemisphere fibers are shown in blue. The fiber tracts for subject $X X$ are shown in figure $7 \cdot 3 \mathrm{~B}$. In two of the control subjects (FK, 114 fibers and TH, 171 fibers), we found a direct SC-AMG-PLV 


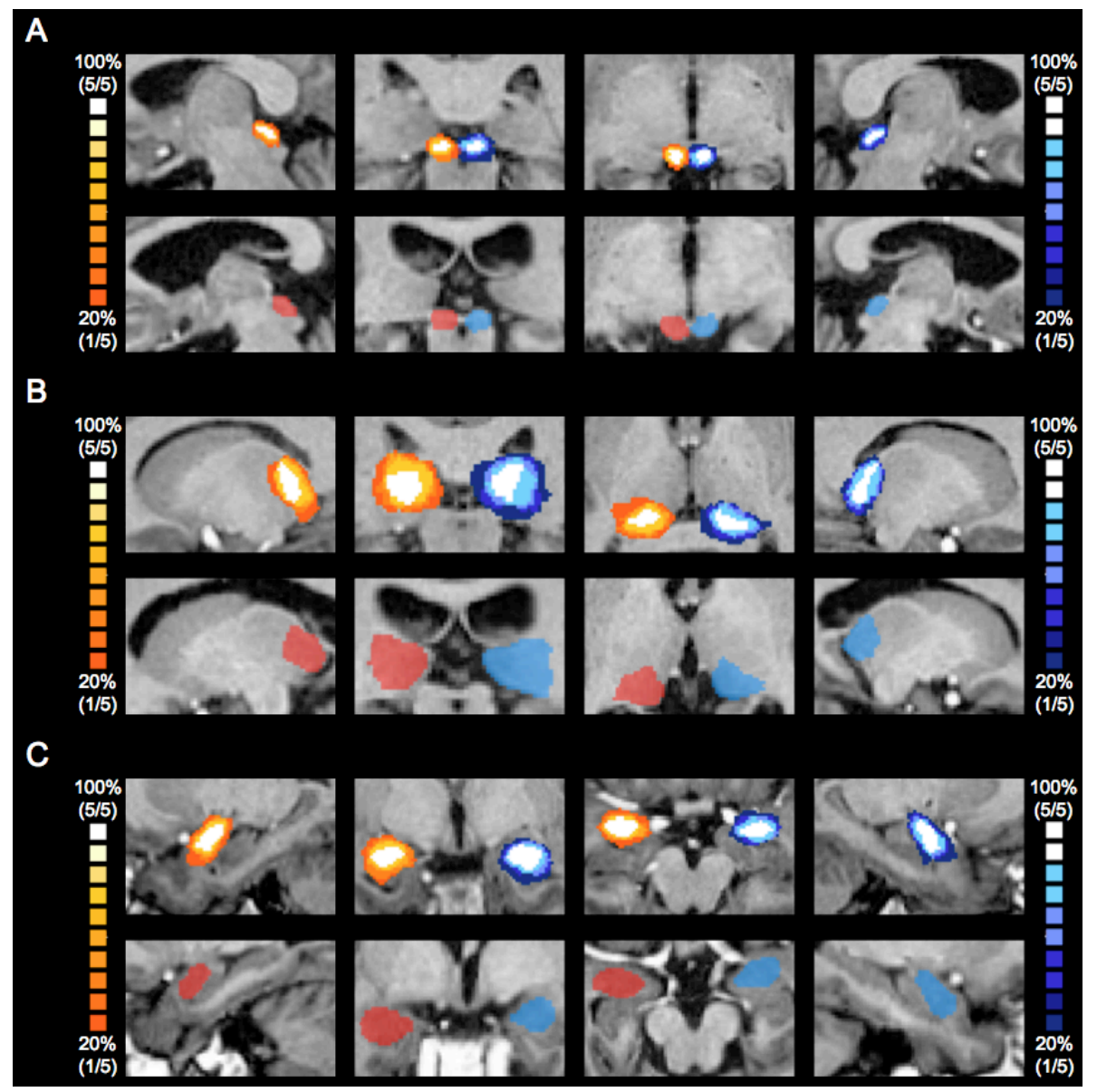

Figure 7.2: ROI definitions in control subjects (top rows in $\mathrm{A}, \mathrm{B}, \mathrm{C}$, left in orange, right in blue) and in GY (bottom row in A, B, C, left in red, right in blue). The ROI definitions are overlapping to a high degree in control subjects. A lighter color indicates a higher degree of overlap. A: Superior Colliculus B: Pulvinar C: Amygdala.

Table 7.2: Volume of manually defined seed regions for fiber tracking in $\mathrm{mm}^{3}$. AMG: amygdala, PLV: pulvinar, SC: superior colliculus.

\begin{tabular}{ccccccc} 
Subject & L AMG & R AMG & L PLV & R PLV & L SC & R SC \\
\hline FK & 1160 & 1528 & 1529 & 1535 & 163 & 176 \\
FS & 1328 & 1896 & 1571 & 1550 & 216 & 227 \\
GL & 1648 & 998 & 1449 & 1471 & 185 & 188 \\
PB & 1136 & 867 & 1441 & 1618 & 167 & 178 \\
TH & 1632 & 2040 & 1433 & 1518 & 188 & 188 \\
GY & 1624 & 1720 & 1506 & 1504 & 255 & 236
\end{tabular}


Table 7.3: Absolute number of fibers found in each connection. SC: superior colliculus, PLV: pulvinar, AMG: amygdala.

Right Hemisphere

\begin{tabular}{lccc} 
Subject & R SC PLV & R PLV AMG & R AMG PLV SC \\
\hline FK & 61187 & 83631 & 114 \\
FS & 37275 & 74022 & 0 \\
GL & 40037 & 70468 & 2 \\
PB & 49111 & 75070 & 8 \\
TH & 33240 & 69154 & 171 \\
GY & 60921 & 91138 & 25 \\
& \multicolumn{2}{c}{ Left Hemisphere } \\
Subject & L SC PLV & L PLV AMG & L AMG PLV SC \\
\hline FK & 47781 & 67418 & o \\
FS & 42027 & 58139 & 4 \\
GL & 39422 & 57297 & 1 \\
PB & 51241 & 70690 & o \\
TH & 25268 & 49591 & 1 \\
GY & 63194 & 92662 & 79
\end{tabular}

connection in the right hemisphere. In TH we found that the connection continued from the pulvinar into the right frontal area. In the other three controls, we found very little or no fibers. In FS we found o, in GL 2 and in PB 8 fibers. In GY, we found 25 fibers connecting SC, PLV and AMG in the right hemisphere.

In the left hemisphere, remarkably, we found little or no fibers in the healthy controls. In FS, GL and TH we found 4, 1 and 1 fiber(s) respectively, while in GY we found 79 fibers connecting SC, PLV and AMG in the left damaged hemisphere.

\section{Overview of separate fiber tracts}

SUPERIOR COLLICULUS-PULVINAR FIBER TRACTS In the control group we found connections in both hemispheres projecting from the superior colliculus to the pulvinar. The superior colliculus connects to the superior colliculus and the pulvinar in the other hemisphere, see figure 7.5 R SC \& PLV and L SC \& PLV.

A 3 -D overview of fiber reconstructions in representative control FS and GY is shown in figure 7.4. The amount of overlap found in the control group is shown in figure $7 \cdot 5$. Only fibers present in at least $40 \%$ of the control group $(2 / 5$ 


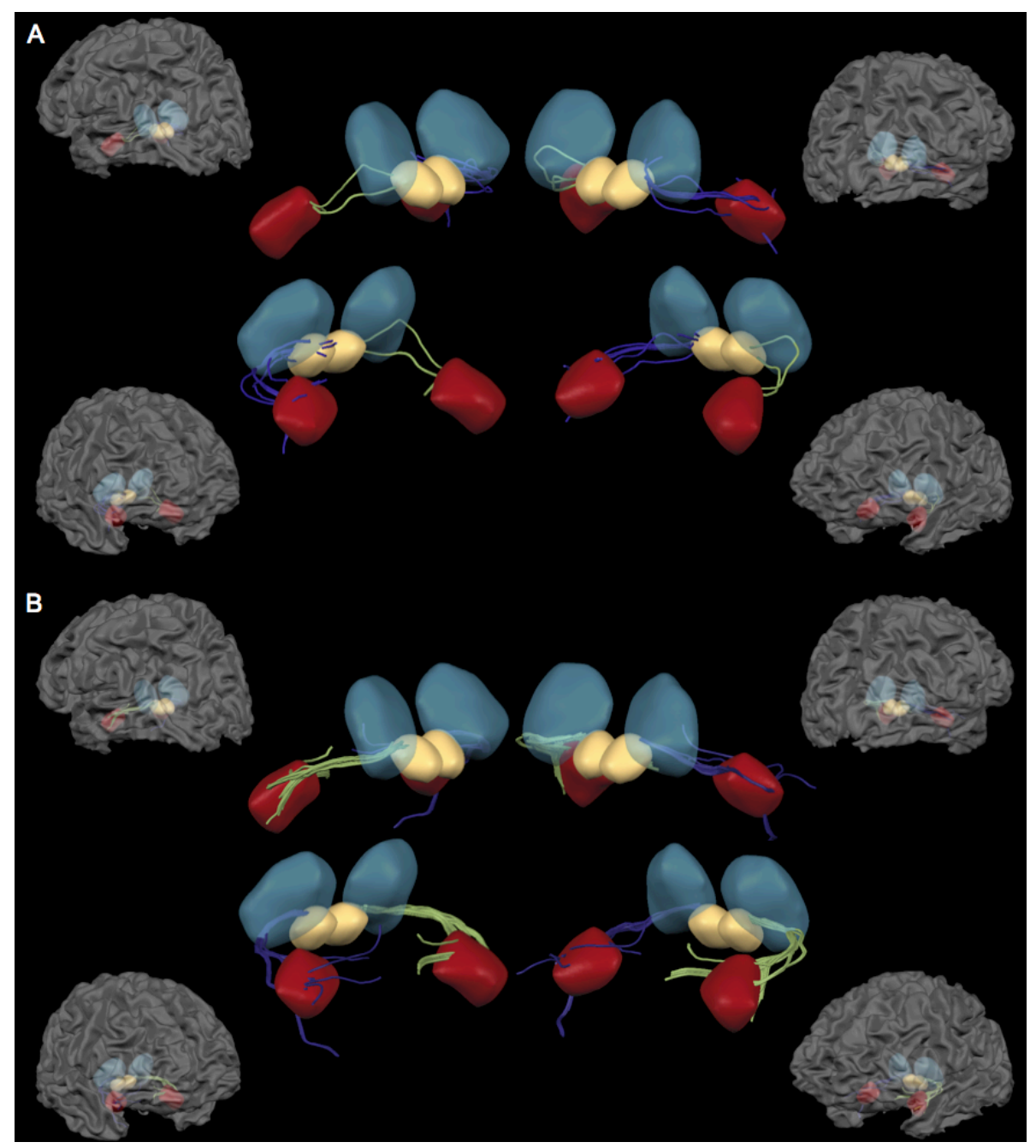

Figure 7.3: Amygdala-pulvinar-superior colliculus fiber reconstructions in A: one representative age-matched control subject and B: GY. Fibers in the left hemisphere in light green, right hemispheric fibers in blue. The superior colliculus is shown in light yellow, pulvinar in light blue and amygdala in red. In the right hemisphere fibers connecting the three regions were found in two of the control subjects, while little or no fibers were found in the right hemisphere. In GY, we found a considerable number of fibers in both the left and the right hemisphere connecting superior colliculus, pulvinar and amygdala.

subjects) are shown. If we look closer within the right hemisphere fibers from the pulvinar project to frontal and pre-frontal areas (caudate and orbitofrontal cortex), posterior parietal areas and occipital visual areas $\mathrm{V}_{1}, \mathrm{~V}_{2}, \mathrm{~V}_{3}, \mathrm{~V}_{4}$ and $\mathrm{MT} / \mathrm{V}_{5}$ and fibers project upwards to primary motor cortex (M1), frontal eye fields (FEF) and dorsal prefrontal cortex. In the left hemisphere, fibers project to occipital visual areas $\mathrm{V}_{1}, \mathrm{~V}_{2} / 3, \mathrm{~V}_{4}$, and to $\mathrm{MT} / \mathrm{V}_{5}$, amygdala, frontal areas cau- 


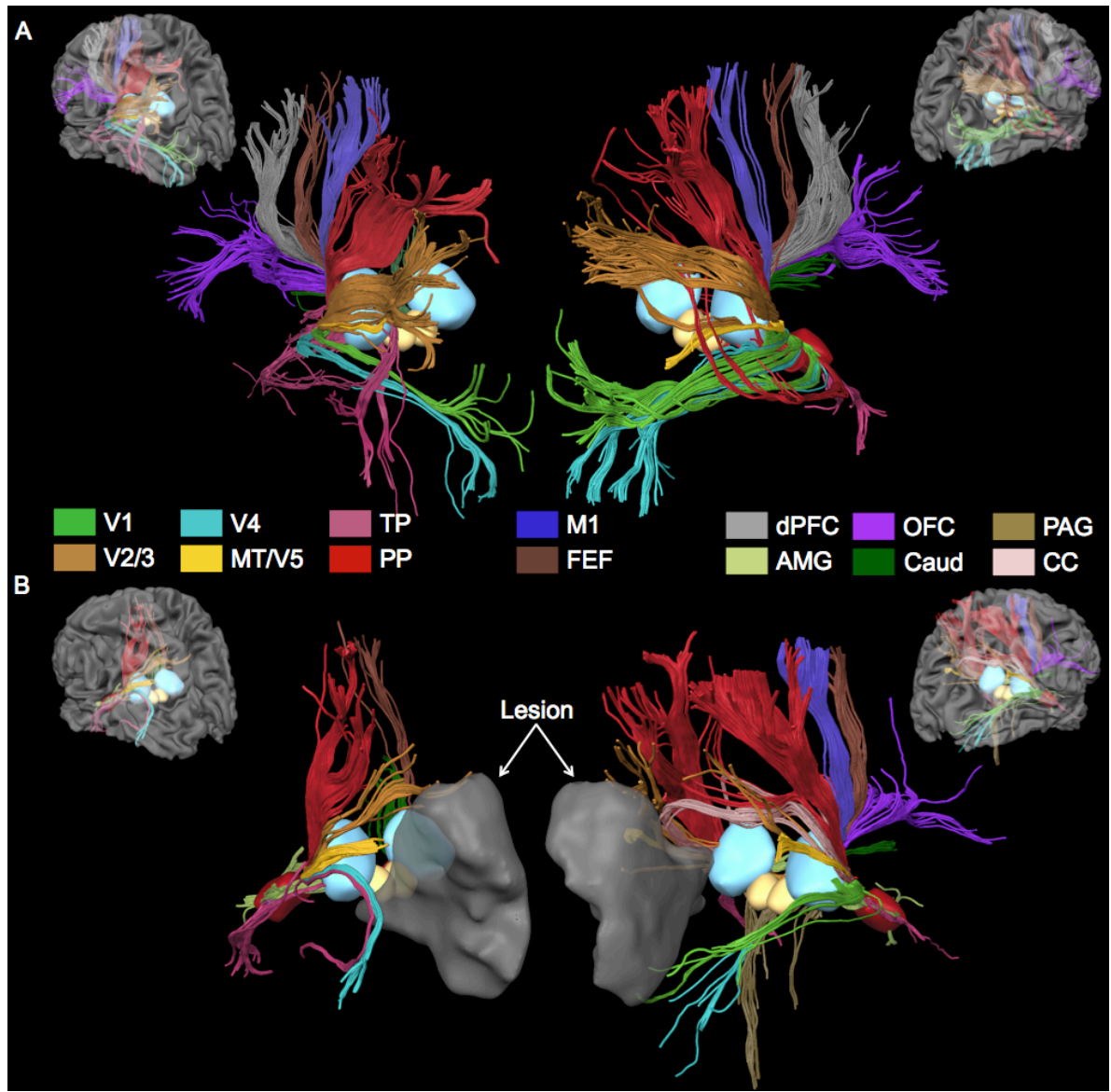

Figure 7.4: Superior colliculus-pulvinar reconstructed fibers in A: control subject FS and B: GY. Reconstructed fibers are color coded according to the areas they project to. $V_{1} / 2 / 3 / 4 / 5$ : Visual areas $V_{1}$ to $V_{5}$ TP: temporal pole PP: posterior parietal cortex Mi: primary motor cortex FEF: frontal eye fields dPFC: dorsal prefrontal cortex AMG: amygdala OFC: orbitofrontal cortex Caud: caudate PAG: periacqueductal gray CC: corpus callosum.

date and orbitofrontal cortex, posterior parietal cortex and upwards to primary motor cortex, FEF and dorsal prefrontal cortex.

Figure $7 \cdot 4 \mathrm{~B}$ shows a 3 -D overview of reconstructed superior colliculus-pulvinar pathways in GY. In GY's right hemisphere, fibers from the superior colliculus project to posterior parietal and frontal areas via the pulvinar. The right superior colliculus connects to the left superior colliculus, left pulvinar and from the pulvinar to left posterior parietal areas. Fibers originating from superior colliculus to pulvinar connect to $\mathrm{V}_{1}, \mathrm{~V}_{2} / 3, \mathrm{~V}_{4}$ and $\mathrm{MT} / \mathrm{V}_{5}$ and periacqueductal gray matter, upwards to posterior parietal areas, FEF and M1. Fibers travel anteriorly into the temporal pole, orbitofrontal cortex, caudate and amygdala. A portion of the fibers travel to the opposite hemisphere via the corpus callosum. The left superior colliculus connects to the left pulvinar, left amygdala and left posterior 


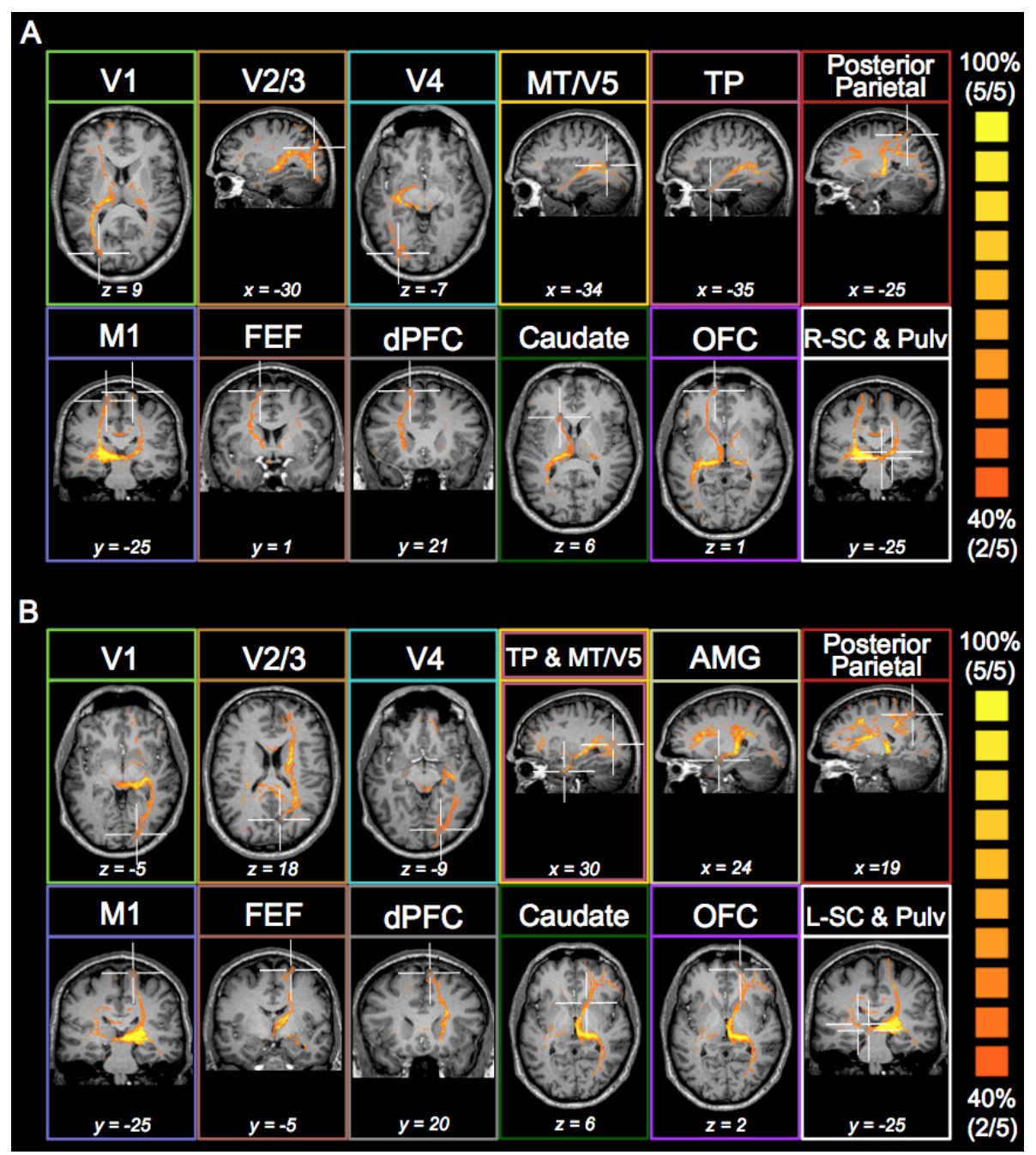

Figure 7.5: Superior colliculus-pulvinar fiber overlap in the control group. Color indicates the amount of overlap, ranging from $40 \%$ (fibers found in at least 2 out of 5 subjects) to $100 \%$ (fibers found in all control subjects). The white cross-hair indicates the termination area of the fibers.

parietal areas. Fibers connect upwards to FEF and frontal into the temporal pole and caudate. Via the pulvinar fibers project towards visual areas $\mathrm{V}_{2} / 3, \mathrm{~V}_{4}$ and $\mathrm{MT} / \mathrm{V}_{5}$.

AMYGDALA - PULVINAR FIBER TRACTS A three-dimensional representation of the fiber tracts found when tracing amygdala-pulvinar connections is shown in figure $7.6 \mathrm{~A}$ for representative subject PB. Figure 7.7 shows the overlap map for all five subjects, only showing fibers present in at least $40 \%$ of the control group (2/5 subjects).. Amygdala-pulvinar fiber tracts in the left hemisphere of the controls terminate in temporal pole, dorsal prefrontal cortex, caudate and orbitofrontal cortex. In the right hemisphere, fiber tracts run towards the tem- 


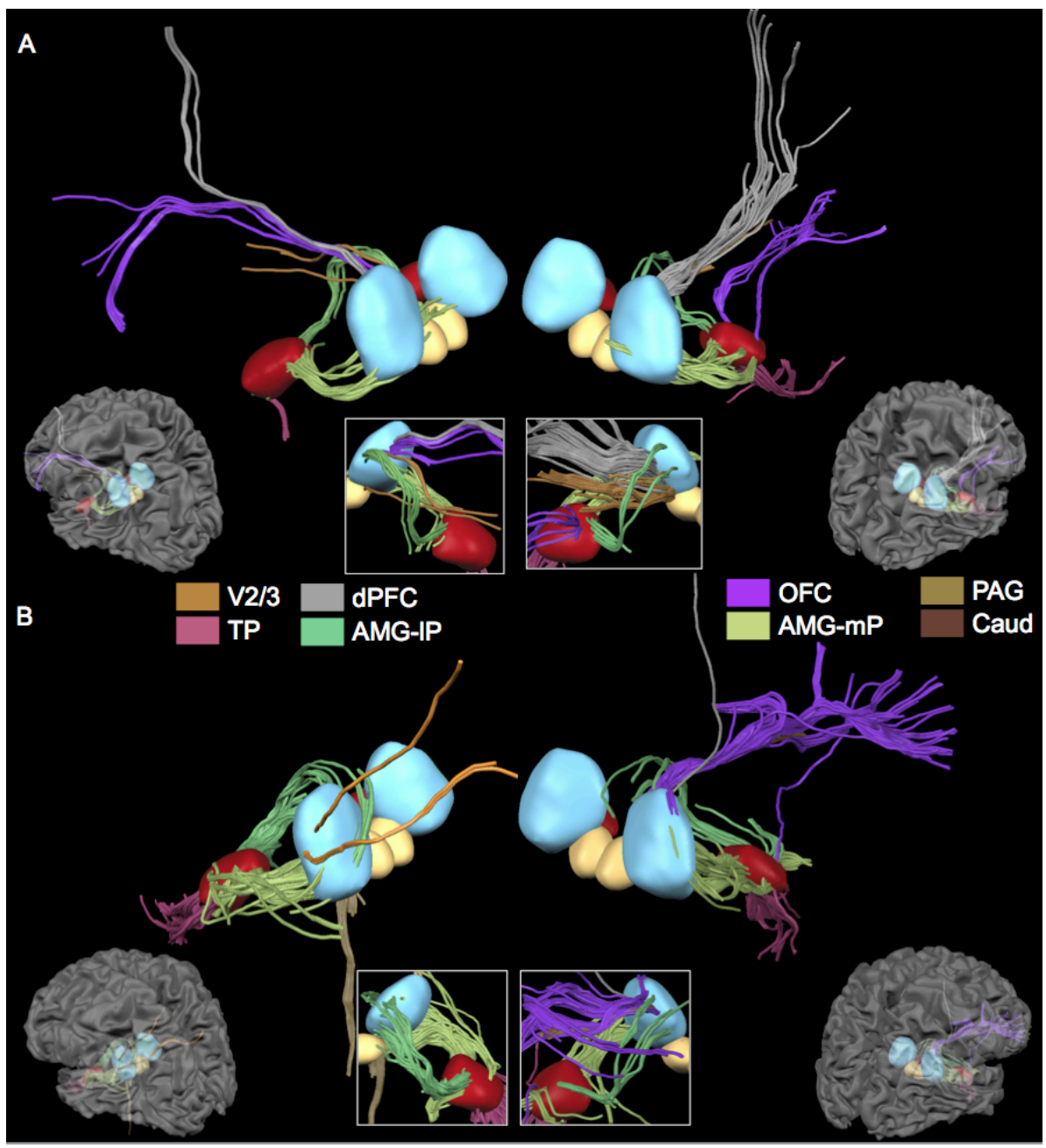

Figure 7.6: Amygdala-Pulvinar fiber reconstructions in A: control subject PB and B: GY. Reconstructed fibers are color coded according to the areas they project to. V2/3: visual areas 2/3 TP: temporal pole dPFC: dorsal prefrontal cortex AMGIP: amygdala projections to inferior-lateral portions of the pulvinar OFC: orbitofrontal cortex AMG-mP: amygdala projections from medial portions of pulvinar PAG: posterior angular gyrus Caud: caudate.

poral pole, dorsal prefrontal cortex, caudate, orbitofrontal cortex and superior colliculus. In two subjects projections from inferior lateral and medial portions of the pulvinar to the amygdala were found in both hemispheres.

Amygdala-pulvinar connections in GY are shown in figure 7.6B. In the left hemisphere, fibers are found projecting into $\mathrm{V}_{2} / 3$, temporal pole periacqueductal gray and to the amygdala from the inferior-lateral and medial portions of the pulvinar via separate routes. In the right hemisphere, fibers connect to orbitofrontal cortex, dorsal prefrontal cortex, temporal pole, and to the amygdala from inferior-lateral and medial portions of the pulvinar. Interestingly, we do 
find fibers projecting from the pulvinar to frontal areas in the right hemisphere of GY but not in the left hemisphere.

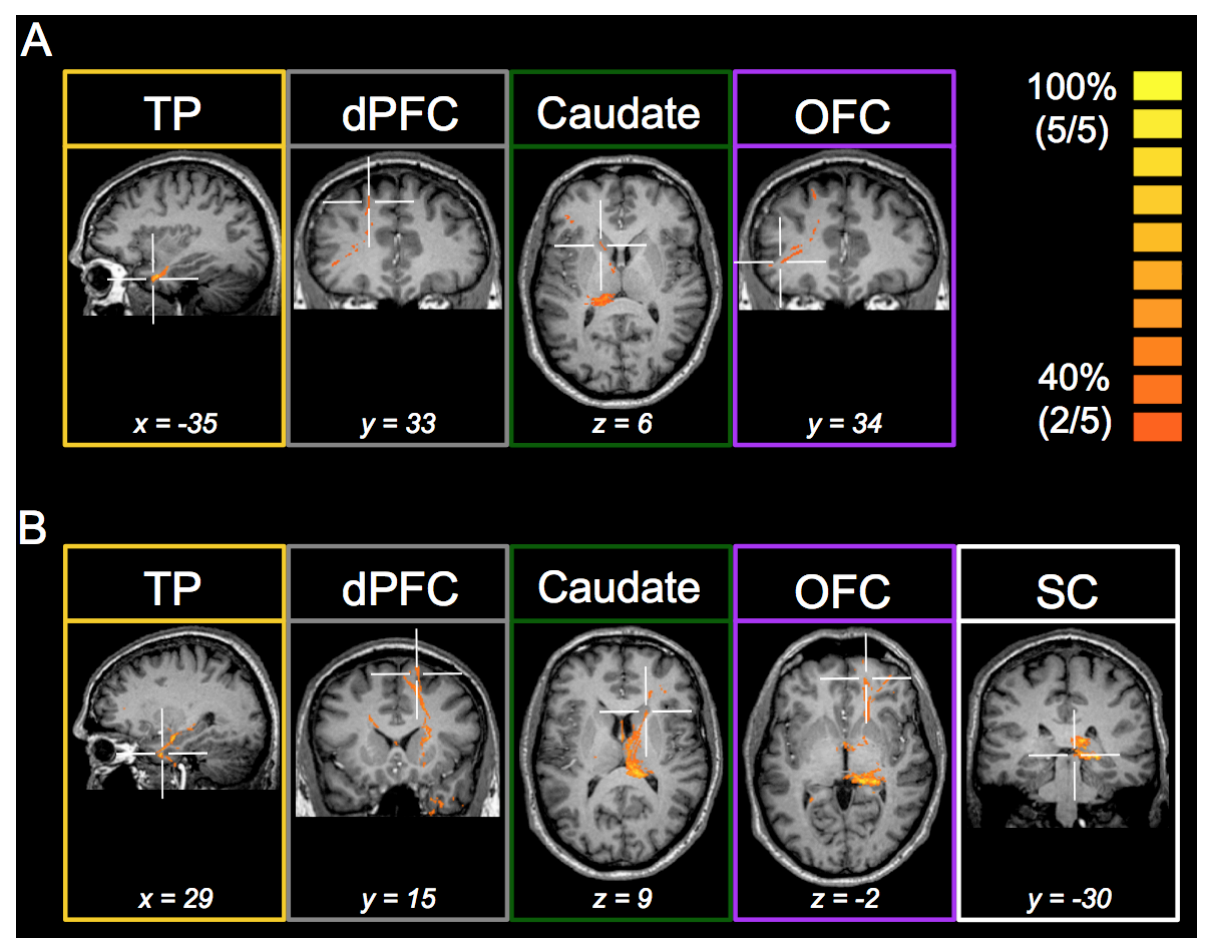

Figure 7.7: Amygdala-pulvinar fiber overlap in the control group.

\section{Connectivity analysis}

The connection strength values for each connection were tested for normality with a Lilliefors test, and were found to fit in a normal distribution (data not shown), so a one-sample $t$-test between the mean value of the controls and GY was appropriate to use.

Figure 7.8 shows that in GY compared to controls, the connection strength in right superior colliculus to/from pulvinar (o.832) is not significantly different from the mean connection strength in the controls (0.813). In the left SC-PLV, GY's connection strength (0.298) is lower, but not significantly different from the mean connection strength in the control group (0.703).

In the right pulvinar to/from amygdala connection strength in GY (0.0236) is not significantly different from than the mean of the control group (0.0392). In the left PLV-AMG, the connection strengths in GY: 0.0569 is significantly larger than the mean controls $0.00842(p<0.05)$.

When we look at the combination of the three regions SC, PLV and AMG in the left hemisphere, the connectivity in GY is significantly larger (0.236) than the mean connectivity in controls (0.0231) at $(p<0.05)$. In the right hemisphere, the 


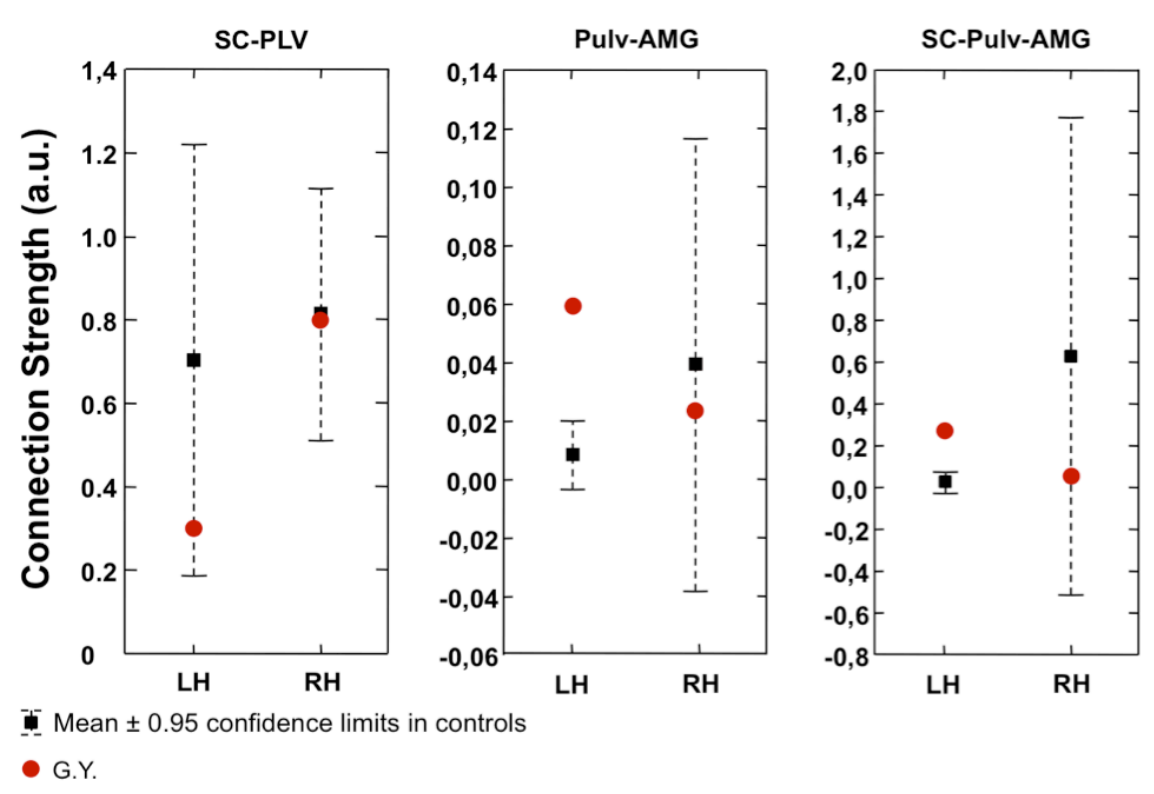

Figure 7.8: Connectivities as calculated by Equation 1. See text for explanation of the box and whisker. The Right Superior Colliculus to/from Pulvinar, Left Pulvinar to/from Amygdala and Left superior colliculus - pulvinar - amygdala connections in GY differ significantly from the controls (one sample $t$-test at $p<0.05)$.

connectivity in GY (o.0237) is not significantly different from the mean connectivity value in controls (o.629).

\section{$7 \cdot 4$ DISCUSSION}

Ample evidence exists for non-consciously perceived emotional visual stimuli in humans [1]. Patients with affective blindsight caused by destruction of the visual cortex (VI) are nevertheless able to correctly judge emotional visual stimuli. In rats [9] and birds [8] there exists a subcortical V1-independent visual pathway from the superior colliculus via the pulvinar to the amygdala. This pathway has not yet been identified in (non-human) primates.

DTI based fiber tracking was used to investigate this supposed subcortical pathway non-invasively in affective blindsight patient GY and 5 healthy control subjects. Direct connections between SC, PLV and AMG in both hemispheres, as well as each individual segment SC to PLV, SC to AMG and PLV to AMG were investigated.

We have found evidence for the existence of a superior colliculus-pulvinaramygdala pathway in the right (non-damaged) hemisphere of GY and 2 of the control subjects. The pathway appears like a loop starting from the SC, connect- 
ing to PLV and AMG and then projecting back from AMG to PLV as we have found by identifying each segment SC-PLV, SC-AMG and PLV-AMG. In the left hemisphere, we have not found such a pathway in the control subjects, but we have been able to identify it in the damaged left hemisphere of GY and it has a similar appearance as in the right undamaged hemisphere. The connection strength of each SC-PLV-AMG segment was computed and this showed that in the left hemisphere of GY, SC-AMG and PLV-AMG connection strengths are significantly higher than in the control subjects. In the right hemisphere, the connection strength between SC and PLV in GY is significantly higher than in the control group.

The connections found from superior-colliculus to pulvinar are in high agreement with connections reported earlier using probabilistic DW-MRI fiber tracking in healthy subjects [19, 20]. SC was found to be connected to visual association areas, parietal-occipital cortex, FEF and prefrontal cortex [19]. Pulvinar was found to have connections to superior colliculus, caudate, FEF, prefrontal areas, posterior parietal areas and $V_{1}, V_{2} / 3, V_{4}$ and $V_{5}$ [20]. In this study, we have focussed on a combined tractography of superior colliculus and pulvinar and we have demonstrated the existence of all before-mentioned tracts in our healthy control group.

In DW-MRI, several pitfalls may arise in acquiring and processing the data [21]. Eddy currents which cause additional gradients on top of the diffusion gradients, cause distortions in spin-echo EPI diffusion weighted images. To reduce the eddy currents and the resulting distortion, we used a double-refocussed spinecho EPI sequence [13]. Additionally, subject motion will affect the data. In our experiments, scan time was $8 \mathrm{~min} 27 \mathrm{~s}$ so subject motion is unlikely to have a large effect. Estimation of the motion parameters in the data did not reveal motion large enough to be corrected for.

Regions of interest defined on the anatomical data of each subject individually show substantial overlap. We have traced the fiber tracts in each subject's individual space and transformed the tracts later into a common Talairach space, thereby avoiding issues that may arise when diffusion data is transformed and interpolated into a common space (e.g. deformation of the shape of the diffusion tensor [22]).

DTI based deterministic fiber tracking has shown its great potential and use in various studies where it is compared with known anatomy e.g. in [23, 24, 25, 26]. It shows that we can achieve good agreement with white matter structures. The error propagating in the fiber tract increases as it extends towards the cortex [27]. In our study we looked at sub-cortical structures deep in the white matter, so we expect the error in the fiber tracts to be relatively low. In comparison to the probabilistic fiber tracking method used in previous studies investigating SC and pulvinar connectivity, deterministic tractography is more conservative, 
that is, determistic fiber tracking finds relatively fewer connections compared to probabilistic FT but with higher connectivity values [17].

Multi-subject comparison of derived parameters of DW-MRI is not a trivial case. To determine the "strength" of a white matter connection, a comparison of the number of fiber tracts found by the algorithm is not reliable, since the number of fibers found directly follows from the number of seedpoints used. We therefore corrected for the size of the seed ROIs [17] to enable a direct comparison of "connection strength" between subjects.

Another possible confound that might explain the differences in the SC-PLVAMG pathway found in the left hemisphere of the controls and the left (damaged) hemisphere of GY is that the presence of a lesion could bias tractography algorithm. Nevertheless, the bias, if present, is not equally distributed over the entire space but is specific for tracts adjacent to, or entering in, the lesion, which is located in left V1. In the present case this bias can be safely discarded because we investigated fiber tracts connecting subcortical structures far away from the cortical lesion. Moreover, to investigate this further we performed additional analyses. First, for each investigated pathway we performed both deterministic tractography from a seed region to the target region as well as the reversed analysis. This showed great convergence in the identified fiber tracts. Second, when a comparison was made between tracts different from the SC-PLV-AMG one, such as the SC-PLV in the left hemisphere, we found remarkable overlapping between the fiber bundles identified in GY and in the control subjects as shown in figure $7 \cdot 5$ and figure $7 \cdot 7$.

Previous work using functional MRI methods revealed a functional connectivity between superior colliculus, pulvinar and amygdala $[3,4]$. It was shown that this functional network is positively activated in response to non-consciously perceived fearful facial expressions in both patients with lesions in the extrastriate cortex. These results suggested the existence of totally subcortical V1independent pathway to process visual emotional signals. In conclusion, we have investigated the subcortical pathways between superior colliculus, pulvinar and amygdala and have found evidence for such a pathway bilaterally in blindsight patient GY.

\section{REFERENCES}

[1] M Tamietto and B de Gelder. Neural bases of the non-conscious perception of emotional signals. Nature reviews. Neuroscience, 11:697-709, 2010.

[2] JE LeDoux. The Emotional Brain. Simon and Shuster, New York, 1996.

[3] JS Morris, A Ohman, and RJ Dolan. A subcortical pathway to the right amygdala mediating "unseen" fear. PNAS, 96(4):1680-5, 1999. 
[4] LM Williams, P Das, BJ Liddell, AH Kemp, CJ Rennie, and E Gordon. Mode of functional connectivity in amygdala pathways dissociates level of awareness for signals of fear. The Journal of neuroscience, 26(36):9264-71, 2006.

[5] BN Pasley, LC Mayes, and RT Schultz. Subcortical discrimination of unperceived objects during binocular rivalry. Neuron, 42:163-172, 2004.

[6] MA Williams, AP Morris, F McGlone, DF Abbott, and JB Mattingley. Amygdala responses to fearful and happy facial expressions under conditions of binocular suppression. J Neurosci, 24:2898-2904, 2004.

[7] KL Yoon, SW Hong, J Joormann, and P Kang. Perception of facial expressions of emotion during binocular rivalry. Emotion, 9:172-182, 2009.

[8] ED Jarvis et al. Avian brains and a new understanding of vertebrate brain evolution. Nature Rev. Neurosci, 6:151-159, 2005.

[9] R Linke, AD De Lima, H Schwegler, and HC Pape. Direct synaptic connections of axons from superior colliculus with identified thalamo-amygdaloid projection neurons in the rat: possible substrates of a subcortical visual pathway to the amygdala. J Comp. Neurol, 403:158-170, 1999.

[10] PJ Basser, J Mattiello, and D Lebihan. MR Diffusion Tensor Spectroscopy and Imaging. Biophysical Journal, 66(1):259-267, 1994.

[11] P Basser, S Pajevic, C Pierpaoli, J Duda, and A Aldroubi. In vivo fiber tractography using DT-MRI data. Magnetic Resonance in Medicine, 44:625$632,2000$.

[12] S Mori, Van Zijl, and P.c. Fiber tracking: principles and strategies-A technical review. NMR Biomed., 15:468-480, 2002.

[13] TG Reese, O Heid, RM Weisskoff, and VJ Wedeen. Reduction of EddyCurrent-Induced Distortion in Diffusion MRI Using a Twice-Refocused Spin Echo. Magnetic Resonance in Medicine, 182:177-182, 2003.

[14] DK Jones, MA Horsfield, and A Simmons. Optimal strategies for measuring diffusion in anisotropic systems by magnetic resonance imaging. Magn. Reson. Med., 42:515-525, 1999.

[15] E Haber and J Modersitzki. Intensity gradient based registration and fusion of multi-modal images. Med Image Comput Comput Assist Interv., 9(Pt 2):726733, 2006.

[16] C Westin, SE Maier, H Mamata, A Nabavi, FA Jolesz, and R Kikinis. Processing and visualization for diffusion tensor MRI. Medical Image Analysis, 6:93-108, 2002. 
[17] T-S Yo, A Anwander, M Descoteaux, and P Fillard. Quantifying Brain Connectivity : A Comparative Tractography Study. Lecture Notes in Computer Science, 5761:886-893, 2009.

[18] J Talairach and P Tournoux. Co-Planar Stereotaxic Atlas of the Human Brain. Thieme Medical Publishers, New York, 1988.

[19] SE Leh, H Johansen-Berg, and A Ptito. Unconscious vision: new insights into the neuronal correlate of blindsight using diffusion tractography. Brain, 129(Pt 7):1822-32, 2006.

[20] SE Leh, MM Chakravarty, and A Ptito. The Connectivity of the Human Pulvinar : A Diffusion Tensor Imaging Tractography Study. Int J Biomedical Imaging, 2008:ID 789539, 2008.

[21] DK Jones and M Cercignani. Twenty-five pitfalls in the analysis of diffusion MRI data. NMR in biomedicine, 23(7):803-20, 2010.

[22] V Arsigny, P Fillard, X Pennec, and N Ayache. Log-Euclidean Metrics for Fast and Simple Calculus on Diffusion Tensors. Magnetic Resonance in Medicine, 421:411-421, 2006.

[23] M Catani, R J Howard, S Pajevic, and DK Jones. Virtual in vivo interactive dissection of white matter fasciculi in the human brain. Neuroimage, 17:77$94,2002$.

[24] S Wakana, Hi Jiang, LM Nagae-Poetscher, PCM van Zijl, and S Mori. Fiber tract-based atlas of human white matter anatomy. Radiology, 230(1):77-87, 2004 .

[25] S Wakana, A Caprihan, MM Panzenboeck, JH Fallon, M Perry, RL Gollub, K Hua, J Zhang, H Jiang, P Dubey, A Blitz, PCM van Zijl, and S Mori. Reproducibility of quantitative tractography methods applied to cerebral white matter. NeuroImage, 36:630-644, 2007.

[26] K Oishi et al. NeuroImage Human brain white matter atlas : Identification and assignment of common anatomical structures in superficial white matter. NeuroImage, 43:447-457, 2008.

[27] M Lazar. Mapping brain anatomical connectivity using white matter tractography. NMR in biomedicine, 23(7):821-35, 2010. 

Since its introduction, DW-MRI and DTI have offered great insight in the white matter organization of the human brain. However, the complex issue of data collection and processing is not free from problems and errors.

DW-MRI is highly sensitive to noise and other artefacts. Typical artefacts include subject motion and eddy current artefacts, but many more artefacts can be present, such as signal dropouts, ghosting artefacts, scanner vibration artefacts and susceptibility related distortions at air-tissue interfaces [1]. Because DWI acquisitions are sensitized to microscopic motions of water molecules, small head movements may induce large artefactual signal changes. Eddy current distortions are mainly the result of the fast switching of large gradients, but they can be reduced by applying double-refocussed gradients [2].

There are many degrees of freedom during the acquisition and analysis of DWI data. As there is currently no standard acquisition protocol for DWI experiments, among the choices the experimenter must make are the b-value, the gradient direction scheme and number of bo weighted images to include. All these factors have their influence on data quality and usability for a certain purpose. For instance, data acquired with a low number of gradient directions (i.e. 3-12) is only suitable for calculation of ADC maps, while at least 20-30 unique gradient directions are needed to reliably estimate the diffusion tensor and fractional anisotropy [3]. For more complex non-gaussian diffusion models the data requirements are more demanding as more than one-hundred gradient directions might be required and there is no consensus on which b-value should be used [4].

The analysis of DWI data requires amongst others assessing data quality, motion and/or eddy current induced distortion correction, estimation of the diffusion tensor, calculating derived scalar measures, region-of-interest analysis and finally fiber tracking. All these steps are sensitive to errors -at least 25 pitfalls in DWI analysis were identified [5]- which in the end will lead to ambiguities and mis-interpretations of the data. The lack of a ground-truth is an important issue in DWI and validation is crucial.

\subsection{VALIDATION AND CORRECTION OF DW-MRI DATA}

The first part of this thesis is dedicated to the validation and correction of DWI data. The goal of Chapter 2 was to develop a hardware phantom platform for validation of DWI acquisition, diffusion models and fiber tracking techniques. Such 
a phantom enables researchers to investigate many of the aforementioned issues and pitfalls in the acquisition and analysis pipeline of DWI data. The phantom was constructed by constraining fibers in shrink tube, similar to the phantom described in [6]. Compared to other hardware phantoms, such as fibers compressed between a positive and negative manifold [7] our type of phantom offers higher diffusion anisotropy. Furthermore, when the fibers are compressed between the positive and negative manifold, it is hard to remove air-bubbles from the phantom. This needs to be done by applying a vacuum to the phantom, which is not necessary in the design presented in this thesis, because when the shrink tube is heated in water to shrink it, air bubbles are removed. A third phantom design, which uses a large number of fibers wrapped around a spool [8] does achieve high packing density but lacks the possibility of creating complex geometries such as kissing or crossing fiber configurations. The downside of the presented design is that the phantom cross-section is relatively small and that susceptibility artefacts may arise near the fiber-shrink tube and tube-water interfaces, which is less of a problem in phantoms that achieve fiber compression without the shrink tubes. Secondly, it has turned out that the true, exact crossing angle of the fibers is hard to control exactly (within single degrees of the angle), because the second piece of shrink tube needed for compression of the center of the phantom will cause the fibers to slightly bend away from the initial position by its compressing force (see figure 2.1). This effect can be controlled by fixating the legs of the phantom during the shrinking stage of the second piece of shrink wrap, but the true local crossing angle could possibly only be determined by using another distortion-free imaging modality with high resolution, e.g. X-ray or CT.

In Chapter 3 we investigated orientation of the phantom fiber bundles relative to the main magnetic field and its influence on diffusion measures. It was demonstrated that there is an orientation-dependent susceptibility effect of white matter, e.g. in $[9,10,11,12]$, but the influence of this phenomenon on diffusion measures is unclear. In human white matter, a change of FA with respect to the angle between fibers and main magnetic field was found, but no clear relation could be established [11]. Using an anisotropic diffusion phantom, orientation-dependent susceptibilities can be easily induced by changing the physical orientation of the spherical phantom container with respect to the main magnetic field. We have found a change in bo signal intensity and diffusion measures as a function of phantom orientation, but it is likely that these changes are exaggerated compared to white matter. The change in signal intensity in the DWI measurement can also be caused by gradient-echo susceptibility effects during the lengthy EPI readout window. Since fast imaging is not necessary in the phantom we could use non-EPI sequences to investigate the changes in diffusion measures. Furthermore, sensitivity for susceptibility increases with higher fields [13], so it will be interesting to study these effects now that high fields ( $7 \mathrm{~T}$ and beyond for human use) are becoming more and more available. 
However despite the issues, this study provides evidence that there is indeed a change in diffusion measures if a fiber bundle is oriented differently with respect to the main magnetic field, which is therefore likely to exist in white matter as well. A carefully designed study in human subjects should be performed to assess the variation of diffusion measures with respect to fiber orientation in the brain.

Chapter 4 focusses on the correction of eddy-current distortions and motion correction in DWI data. As stated before, eddy currents arise due to rapid changes in large amplitude gradient fields, inducing currents in the scanner's metal parts and thereby generating additional gradients. These gradients distort the image. Unlike global affine methods which co-register the DWI data to the bo image, we implemented the slice-based iterative cross-correlation (ICC) algorithm and made some improvements which make the algorithm better suited for general usage. The original ICC algorithm could only be used for small bvalues $\left(<300 \mathrm{~s} / \mathrm{mm}^{2}\right)$ [14]. In this thesis, the ICC algorithm could be extended to be used to model distortions for data collected at higher b-values by using the parameters for gradient directions and gradient strength to model distortions.

The eddy-current correction method was combined with a motion correction procedure and can be run iteratively. The correction quality was checked with a PCA decomposition of the corrected data. In comparison with FSL-FLIRT, the first PCA component of our method was slightly lower, but upon visual inspection of the higher components FSL-FLIRT showed an overestimate of the distortions in the readout direction as was reported earlier [15].

\subsection{APPLICATIONS OF DW-MRI IN LKS AND BLINDSIGHT}

In the second part of this thesis, we used fMRI, DTI and fiber tracking to investigate a rare condition in children, Landau-Kleffner Syndrome, which is defined by acquired epileptic aphasia. After initial normal development, language capabilities degenerate rapidly in a period of weeks to months due to epileptic seizures (mostly during sleep). If no treatment or therapy is given, language outcome is poor and children are very likely to develop behavioural problems due to a lack of communication. The underlying mechanisms in LKS are not yet understood, since there is not enough neuroanatomical and neurophysiological knowledge to explain the language deterioration. In Chapter 5 multi-modal integration of speech sounds and letters was investigated in a recovered LKS patient. This is, to our knowledge, the first detailed fMRI study in LKS on this early level of language processing. It was found that letter-sound integration is similar to that in normal subjects, but that the right auditory cortex has a much lower response to speech sounds than the left auditory cortex, while the audiogram is normal in both left and right ears. 
In Chapter 6 we investigated the white matter structures involved in hearing and language in a recovered LKS patient. The white matter language tracts (arcuate fasciculi) have been studied to great extend in healthy subjects $[16,17,18$, $19,20]$, and the AF is present in healthy subjects in both the left and in a minority of the subjects in the right hemisphere [17]. Secondly, a segment of the left AF projects into the inferior parietal lobe in healthy subjects. A homologue connection is also present in the right hemisphere of some of the subjects [21]. In the investigated case however, this particular segment was not found in the left hemisphere, but only in the right hemisphere. The cortical region in the inferior-parietal area is related to motor-sensory integration and short-term verbal memory. LKS patients usually have great difficulties acquiring new vocabulary and perform poorly in a dichotic listening task. Since there is no evidence for a connection to this area, as the data indicates in this case, this observation might explain the clinical deficits in LKS. A greater understanding of the functional and neuro-anatomical changes in LKS patient will hopefully contribute to better therapies and reduce the need for highly invasive subpial transection surgery, which in any case does not necessarily lead to restoration of language functions [22].

A major issue in fiber tracking is the exact definition of seed points. The seed region needed to reliably track the AF is well documented and relatively easy to delineate on a color-coded FA map. Since this is a single-case study, we have to rely on information on the AF in the literature. One way to accurately compare the reconstructed tracts to a healthy group of subjects is to use atlases. The atlas we used is based on 40 healthy subjects [23] and can therefore be considered as a reliable atlas if we investigate large well known fiber tracts, although there are differences in data acquisition and analysis software. Furthermore, the atlas data is available and can be directly compared with our single case data. A superior approach to atlas-based comparisons is to obtain DWI data from a homogeneous group of (recovered) LKS patient and compare this to a matched control group.

Such a more direct comparison between five healthy controls and a patient suffering from blindsight, was used in Chapter 7 to study fiber tracts between superior colliculus, pulvinar and amygdala. These structures show higher activation, that is, an increased BOLD response, when emotional visual signals are non-consciously perceived [24]. In animals a direct pathway between amygdala, pulvinar and superior colliculus is shown to exist, but has not been found in humans until now [24]. Since it is hard for healthy subjects to non-consciously perceive visual emotional stimuli, we have conducted a study in a blindsight patient. In this patient, the visual cortex is destroyed, but he is still able to make accurate judgements about emotional stimuli presented visually. We have demonstrated the existence of a direct pathway bilaterally in the blindsight patient, which was not present in the left hemisphere of the control subjects. In the right hemisphere we have found the pathway in two out of five controls. We have also evaluated 
the "connection strength" of the fiber bundles between the three subcortical regions and have found that it is larger in the blindsight patient for the superior colliculus-amygdala and amygdala-pulvinar connections in the left hemisphere, and superior colliculus-pulvinar connections in the right hemisphere.

The key issue in this study is the exact placement of the seed ROIs, which were defined on the T1-weighted dataset instead of the DWI data set. The connectivity calculations are directly influenced by the size of the defined seed regions, but are a better measure than taking the number of fiber tracts originating from the region, since the number of fiber tracts directly follows from the amount of seed points.

While we observed substantial differences between a patient and healthy controls in both fiber tracking studies there is the risk to report false positives (discovering tracts that actually do not exist) or false negatives (existing white matter fibers are not detected). This is a general problem of fiber tracking and is hard to resolve, especially when we look at unknown or undocumented white matter structures and the problem becomes even larger if it involves diseased or damaged brains.

\subsection{RECOMMENDATIONS \& FUTURE DIRECTIONS}

The process of acquiring and processing of DWI data is not error-free, suffers from low SNR and has a lot of degrees of freedom during all steps in the process. Recently, 25 pitfalls were defined on the analysis and acquisition alone [5]. DWI data contains a wealth of information and requires expert knowledge to process. However, it remains to be an elegant methodology that produces new insights in brain anatomy and constitutes the only way to study the microstructural directionality of white matter in-vivo. Some key points to address in the future are:

STANDARDIZATION OF ACQUISITION PROTOCOLS When DWI data is acquired, the $b$ value and the number of diffusion gradients have to be specified. The b-value is usually taken to be $600-1200 \mathrm{~s} / \mathrm{mm}^{2}$, but is dependent on the SNR, echo time and eddy currents [1]. However, an optimal acquisition protocol is highly dependent on technological advances such as better MR pulse sequences, improved hardware (particularly high gradient strength) and scanners with higher magnetic fields (7 T and beyond). Based on simulations, around 2030 unique gradient directions have to be used to correctly estimate FA [3]. The rest of the imaging time may be filled with more gradient directions, more bo images or repetitions of the measurement. The phantom described in chapters 2 and 3 will be of great help to test different acquisition protocols in order to select the one with highest quality in a given setup. A first use of the phantom to 
calibrate optimal acquisition parameters in HARDI is demonstrated in [4] but has not lead to uniform protocols for HARDI yet.

AN ANAlysis of the differences between scanners Comparing the performance of different scanners is of great importance in multi-centre studies using devices from different manufacturers, with different RF coils. In addition to differences in hardware between centres, slight difference between DWI pulse sequences will also influence the measurement. Again, a phantom will be of great help balance differences between sites.

DEVELOPMENT OF QUALITY ASSURANCE (QA) PROTOCOLS QA is necessary to evaluate a scanner's stability over time. A commonly used QA protocol [25] for fMRI includes gradient-echo EPI measurements to test temporal stability, which is crucial for use in fMRI. For DWI, the scanner hardware, particularly the gradients, is driven closer to its limits which makes it more susceptible to acquisition errors and stability problems. Therefore, it is relevant to create specific QA procedures for DWI, where again phantom measurements could play a crucial role. At ultra-high fields such as 7 or $9.4 \mathrm{~T}$, scanner stability is both more essential and at higher risk, so it becomes even more important to develop good DWI QA procedures.

UNIFORMITY OF SOFTWARE ANALYSIS STEPS. UNIFORM ANALYSIS BETWEEN DIFFERENT SOFTWARE PACKAGES There are numerous DWI data analysis softwares around. Each software package for DWI data analysis (BrainVoyager ${ }^{1}$, FSL $^{2}$, DTIStudio ${ }^{3}$, ExploreDTI ${ }^{4}$, MedInria ${ }^{5}$, DTITool ${ }^{6}$ to name a few) has its own approach on processing the data. Although differences may be subtle, there is currently no in-depth analysis in results. There is much to gain from a general standard pipeline, starting with data inspection, distortion and motion correction at least up to the point of tensor estimation.

IMPROVEMENTS IN CO-REgISTRATION AND TEMPLATE MATCHING Coregistration between subjects is commonly done by either aligning the DWI images themselves or to align the DWI images to a TI-weighted anatomical data set. However, these methods were not specifically developed to be used with DWI data and may cause white matter misalignment [5]. If for instance the FA map of each subject is used for co-registration, better alignment may be achieved [5]. The same holds for template matching, to Talairach or MNI space. If templates

\footnotetext{
1 http://www.brainvoyager.com/

2 http://www.fmrib.ox.ac.uk/fsl/

3 https://www.dtistudio.org/

4 http://www.exploredti.com/

5 http://www-sop.inria.fr/asclepios/software/MedINRIA/

6 http://bmia.bmt.tue.nl/software/dtitool/
} 
are used that were specifically developed for DWI, like FSL's FMRIB58_FA7 or the JHU DTI database ${ }^{8}$, better results will be achieved.

DEVELOPING A CLEAR DEFINITION OF FIBER-TRACT BASED CONNECTION STRENGTH An interesting topic is whether or not the "strength of a connection" between two cortical areas based on DTI fiber tractography can be computed. There are thousands of axons penetrating a typical DWI voxel of $8 \mathrm{~mm}^{3}$ and only a single diffusion tensor represents these axons. The more axons in an axonal bundle connecting one cortical region to the other, the more information can be transferred between those regions. This may be reflected in neighbouring DWI voxels if they have similar tensors pointing in the same direction, which then can be reconstructed into a fiber bundle. The question is then if more reconstructed bundles also mean that there is a stronger connection or whether the bundle is able to tranfer more information.

On the other hand, a single tensor might also represent less axons in a voxel, but with a larger diameter. A larger axonal diameter increases the conduction velocity and information might transfer faster, which could be identified as a "stronger" connection.

Secondly the length of a reconstructed fiber might have consequences for the connection strength. The chance of a connection terminating further away from its starting point is less than when it terminates closer to its origin.

The third factor contributing to connection strength is the amount of seed points or the area or volume of the seed region and the number of reconstructed fiber tracts that actually reaches the termination area. A higher number of tracts originating from the seed region will likely give a higher chance of at least some tracts reaching the termination region. The inverse holds as well: the size of the termination area determines how many fiber tracts reach it from the source seed region.

THE USE OF MUltiple models (GaUssian AND NON-GAUSSian) in THE SAME DATA SET Use of non-gaussian diffusion models side-by-side with the conventional gaussian diffusion model will help identifying complex structures in the brain and also will aid the researcher in understanding why the fiber tracking method has chosen a certain path or was prematurely stopped because of for instance low FA values. Combined visualisation of both DTI and nongaussian models is crucial for this technique to be useful and was presented resently [26]. 


\subsection{CONCLUSIONS}

In this thesis we have shown that there is a need for accurate validation of DWI acquisition and processing. The developed hardware phantom can be of great potential to achieve this goal. We have demonstrated for the first time some of the neural correlates underlying Landau-Kleffner Syndrome and we have shown evidence for the existence of a subcortical white matter pathway for non-conscious detection of visual emotional stimuli in a patient suffering from blindsight. However, as pointed out before, the data is complex to acquire and analyse and the possibility of reporting fibers that do not exist or missing fibers that do exist cannot be completely ruled out. Therefore it is appropriate to say in conclusion that, "despite the improvement of diffusion weighted MRI available to us nowadays, there is still more than one obscure point to clarify.." (modified from Déjérine, 1895).

\section{REFERENCES}

[1] J-D Tournier, S Mori, and A Leemans. Diffusion tensor imaging and beyond. Magn Reson Med, 2011.

[2] TG Reese, O Heid, RM Weisskoff, and VJ Wedeen. Reduction of EddyCurrent-Induced Distortion in Diffusion MRI Using a Twice-Refocused Spin Echo. Magnetic Resonance in Medicine, 182:177-182, 2003.

[3] DK Jones. The effect of gradient sampling schemes on measures derived from diffusion tensor MRI: a Monte Carlo study. Magn Reson Med, 51:807815,2004 .

[4] V Prčkovska, A Roebroeck, W Pullens, A Vilanova, and H. Ter Haar Romeny. Optimal Acquisition Schemes in High Angular Resolution Diffusion Weighted Imaging. MICCAI, LNCS 5242:9-17, 2008.

[5] DK Jones and M Cercignani. Twenty-five pitfalls in the analysis of diffusion MRI data. NMR in biomedicine, 23(7):803-20, 2010.

[6] E Fieremans, Y De Deene, S Baete, and I Lemahieu. Design of Anisotropic Diffusion Hardware Fiber Phantoms. Diffusion Fundamentals, 10:3-5, 2009.

[7] C Poupon, B Rieul, I Kezele, M Perrin, F Poupon, and J-F Mangin. New Diffusion Phantoms Dedicated to the Study and Validation of High-AngularResolution Diffusion Imaging ( HARDI ) Models. Magnetic Resonance in Medicine, 1283(October 2007):1276-1283, 2008.

[8] FB Laun, B Stieltjes, S Huff, and LR Schad. Investigations of a dti -phantom with properties similar to in vivo neuronal tissue. Proc Intl Soc Mag Reson Med, page 1526, 2007. 
[9] CJ Wiggins, V Gudmundsdottir, D Le Bihan, V Lebon, and M Chaumeil. Orientation Dependence of White Matter $\mathrm{T}^{*}$ Contrast at $7 \mathrm{~T}$ : A Direct Demonstration. Proc ISMRM, 16:237, 2008.

[10] J Lee, K Shmueli, M Fukunaga, P Van Gelderen, H Merkle, AC Silva, and JH Duyn. Sensitivity of MRI resonance frequency to the orientation of brain tissue microstructure. PNAS, 107:5130-5, 2010.

[11] A Cherubini, P Péran, GE Hagberg, AE Varsi, G Luccichenti, C Caltagirone, U Sabatini, and G Spalletta. Characterization of white matter fiber bundles with $\mathrm{T}_{2}^{*}$ relaxometry and diffusion tensor imaging. Magn Reson Med, 61:1066-72, 2009.

[12] B Bender and U Klose. The in vivo influence of white matter fiber orientation towards $\mathrm{B}(\mathrm{O})$ on $\mathrm{T}_{2}{ }^{*}$ in the human brain. NMR in biomedicine, 23:10711076, 2010.

[13] K Uludag, B Müller-Bierl, and K Ugurbil. An integrative model for neuronal activity-induced signal changes for gradient and spin echo functional imaging. NeuroImage, 48(1):150-65, 2009.

[14] JC Haselgrove and JR Moore. Correction for distortion of echo-planar images used to calculate the apparent diffusion coefficient. Magnetic Resonance in Medicine, 36(6):960-964, December 1996.

[15] S Muñoz Maniega, ME Bastin, and PA Armitage. A quantitative comparison of two methods to correct eddy current-induced distortions in DT-MRI. Magn Reson Imaging, 25(3):341-9, 2007.

[16] M Catani, DK Jones, and DH fFytche. Perisylvian language networks of the human brain. Annals of neurology, 57(1):8-16, January 2005.

[17] M Catani, MPG Allin, M Husain, L Pugliese, MM Mesulam, RM Murray, and DK Jones. Symmetries in human brain language pathways correlate with verbal recall. PNAS, 43(23):17163-17168, 2007.

[18] S Frey, JSW. Campbell, GB Pike, and M Petrides. Dissociating the human language pathways with high angular resolution diffusion fiber tractography. Journal of Neuroscience, 28(45):11435-11444, 2008.

[19] MF Glasser and JK Rilling. DTI Tractography of the Human Brain's Language Pathways. Cerebral Cortex, 18:2471-2482, 2008.

[20] AD Friederici. Pathways to language : fiber tracts in the human brain. Trends in Cognitive Sciences, 13:175-181, 2009. 
[21] A Gharabaghi, F Kunath, M Erb, R Saur, S Heckl, M Tatagiba, W Grodd, and H-O Karnath. Perisylvian white matter connectivity in the human right hemisphere. BMC Neuroscience, 6:10-15, 2009.

[22] JH Cross and B Neville. The surgical treatment of Landau-Kleffner syndrome. Epilepsia, 5o(suppl 7):63-67, 2009.

[23] M Thiebaut de Schotten, DH Ffytche, A Bizzi, F Dell'Acqua, M Allin, M Walshe, R Murray, SC Williams, DGM Murphy, and M Catani. Atlasing location, asymmetry and inter-subject variability of white matter tracts in the human brain with MR diffusion tractography. NeuroImage, 54:49-59, 2011.

[24] M Tamietto and B de Gelder. Neural bases of the non-conscious perception of emotional signals. Nature reviews. Neuroscience, 11:697-709, 2010.

[25] RM Weisskoff. Simple measurement of scanner stability for functional NMR imaging of activation in the brain. Magn Reson Med, 36(4):643-5, 1996.

[26] V Prčkovska, THJM Peeters, M van Almsick, B Ter Haar Romeny, and A Vilanova I Bartroli. Fused DTI/HARDI Visualization. IEEE T. Vis. Comput. Gr., pages 1-13, 2010. 
The white matter in the human brain is highly organized. Distant brain areas are connected to each other by bundles of axons organized in fascicles. Before the advent of diffusion-weighted MRI, brain scientists, anatomists and neurosurgeons had to rely on brain dissection techniques or axonal staining to study the white matter architecture which is tedious time-consuming work and can only be done ex-vivo.

Diffusion-weighted MRI (DW-MRI) can be used to study the white matter architecture non-invasively and thus enables us to study the brain structure invivo. In this thesis approaches for validation, quality assurance and correction of DW-MRI data are proposed and DW-MRI based tractography is applied in conjunction with fMRI to study Landau-Kleffner Syndrome, and to investigate sub-cortical pathways in blindsight with tractography.

To investigate the problems arising in DW-MRI acquisition, post-processing and fiber tracking it is very useful to have a gold standard to validate against. We present an anisotropic diffusion phantom, which can be used, among others, to test DW-MRI sequences, to do quality control and to test models for the reconstruction of diffusion profiles. The phantom contains tightly packed polyester fibers wrapped in a shrink tube aimed at mimicking the diffusive properties of human white matter, and was designed to be measured with a clinical DW-MRI protocol. We have shown that the phantom indeed possesses these properties and that it is possible to do fiber tracking on the data obtained from the phantom.

While analysing the data of such a phantom we discovered a signal deviation which seemed to be orientation-dependent. The signal of the non-diffusion weighted image is higher when the phantom is oriented parallel to the magnetic field than when the phantom is oriented perpendicular to the magnetic field. The diffusion-weighted signal is also affected by this phenomenon and the diffusion measures such as mean diffusivity (MD) and fractional anisotropy (FA) show a dependence on the orientation of the phantom to the magnetic field. It is known from previous work that the orientation of an object containing any structure has an influence on the magnetic field. The local magnetic field (susceptibility) a spin experiences is affected by the surrounding structure or tissue. It is also known that a susceptibility change affects the diffusion signal, so we hypothesise that an orientation-dependent susceptibility change is the main cause of the observed effects.

Due to the large gradients and the rapid switching of these gradients additional currents are induced in the metal parts of the MRI scanner. These currents, 
called Eddy Currents, actually change the shape of the gradient waveform and as a result of that distortions are introduced in the diffusion-weighted images. The eddy currents may be reduced by using a double-refocussed spin-echo diffusion weighted sequence, but post-processing might still be needed to find and correct for distortions in the images. A second issue in the DW-MRI acquisition is subject motion. Since the signal obtained is originating from very small motions of the spins, even small head-movements might influence or corrupt the signal. In this thesis an iterative post-processing method is presented to correct for both motion and eddy current distortions. The method was compared to a standard eddy current and motion correction method and showed to have a small but significant improvement in performance.

In this thesis two application studies of DW-MRI are presented. In the first study a rare syndrome, Landau-Kleffner Syndrome, is investigated. After initial normal development, patients suffering from the syndrome lose their ability to use and understand language and sometimes even to understand natural sounds in a very short period of time (weeks to months). The syndrome presents itself between the age of 3-7 years. Patients suffer from epilepsy, also often during sleep, which disappears when the patient reaches adulthood. Verbal communication may be restored using visual communication consisting of pictograms, finger spelling and/or sign language. Not much is known to date about the neural mechanisms of the syndrome, but it is hypothesized that there is an impairment in the auditory circuit.

The presented case is a 27 year old woman after the epileptic phase. An fMRI study was performed investigating the integration of written letters and speech sounds in the brain. It was found that in the left primary auditory cortex the response to auditory letter sounds is much less pronounced than in the right primary auditory cortex, which is not found in healthy controls. Moreover, we found that the integration of letters and sounds in the superior temporal gyrus and sulcus is comparable to that found in normal healthy subjects, which suggest that the impairment exists in higher language areas. The white matter connections in the patient were also investigated using DW-MRI. In both hemispheres the arcuate fasciculus connecting the primary auditory cortex to language interpretation areas was found. However, in the left hemisphere we were not able to reconstruct the short segments of the arcuate fasciculus, which connect sensorymotor integration areas and verbal short term memory areas to the language centers. This connection is consistently found in healthy subject in the left hemisphere. In the right hemisphere of the patient, we did find the short segments, which are typically not found in the majority of healthy subjects. The results might therefore indicate that the impairment in short-term verbal memory found in Landau-Kleffner patients has its origin in a disconnection in the white matter connecting language areas with sensory-motor integration and short term verbal memory areas. 
In the last study presented in this thesis we investigate the white matter connections presumably related to unconscious perception of emotional visual stimuli. In blindsight patients, in whom the visual cortex is lesioned by an accident or infarct, it was shown that they are able to unconsciously perceive emotional visual stimuli, bypassing the visual cortex. It is hypothesized that instead they are using a sub-cortical system constituting of the amygdala, pulvinar and superior colliculus. This system was earlier found to exist in non-human primates but not yet in humans. In a blindsight patient and five healthy controls we found evidence for the existence of an amygdala-pulvinar-superior colliculus connection using DW-MRI based fiber tracking. Interestingly this connection in the blindsight patient was stronger than in the healthy subjects. The results might indicate possible sub-cortical plasticity and reorganization between these subcortical regions as a result of visual cortex destruction. 
De witte stof in de menselijke hersen is zeer goed georganiseerd. Hersengebieden zijn met elkaar verbonden door fasciculi ofwel bundels van axonen.

Voordat diffusie-gewogen MRI ten tonele kwam moesten neurowetenschappers, anatomen en neurochirgurgen het doen met dissecties van de hersen of het aankleuren van axonen om de architectuur van de witte stof te doorgronden, wat zeer veel tijd kost en alleen maar ex-vivo gedaan kan worden.

Diffusie-gewogen MRI (DW-MRI) kan gebruikt worden om de witte stof in de hersenen non-invasief te bestuderen en zo in-vivo de structuur van de hersenen bloot te leggen. In dit proefschrift worden methoden voorgesteld om DW-MRI data te valideren, kwaliteitscontrole te doen en te corrigeren. Op DW-MRI data gebaseerde tractography wordt toegepast, samen met fMRI, om het LandauKleffner syndroom te bestuderen en om sub-corticale wittestofbanen in blindsight te onderzoeken.

Om de problemen die ontstaan in de acquisitie, nabewerking en fiber tracking van DW-MRI data te onderzoeken is het zeer waardevol om een gouden standaard te hebben waartegen gevalideerd kan worden. In dit proefschrift wordt een kunstmatig model, een anisotroop diffusiefantoom gepresenteerd, dat onder andere gebruikt kan worden voor het testen van diffusiesequenties, om kwaliteitscontroles uit te voeren en om de nieuwe modellen voor het reconstrueren van diffusieprofielen te testen. Het fantoom bevat dichtgepakte polyester vezels omhuld door een krimpkous en er is geprobeerd de diffusie-eigenschappen van witte stof te benaderen. Het kan gebruikt worden met een klinisch DW-MRI scanprotocol. We hebben aangetoond dat het fantoom deze eigenschappen inderdaad bezit en dat het mogelijk is fiber tracking te doen op de data afkomstig van het fantoom.

Toen we de data afkomstig van het fantoom analyseerden, ontdekten we een afwijking in het signaal die orientatie-afhankelijk leek te zijn. Het signaal in het niet-diffusiegewogen beeld is hoger als het fantoom parallel aan het magnetisch hoofdveld ligt, dan wanner het loodrecht daarop ligt. Het diffusie-gewogen signaal wordt ook beïnvloed door dit fenomeen en de diffusiematen "mean diffusivity" (gemiddelde diffusiviteit) en fractionele anisotropie (FA) laten een verandering zien als gevolg van de orientatie ten opzichte van het magnetisch hoofdveld. Uit de literatuur is het bekend dat de oriëntatie van een object dat enige structuur bezit invloed heeft op het magnetisch veld. Het locale magnetisch veld (susceptibiliteit) dat een spin voelt wordt beïnvloed door de omliggende structuren of weefsel. Het is ook bekend dat een verandering in susceptibiliteit de diffusiewaarde verandert, dus we stellen dat de oriëntatie-afhankelijke susceptibiliteit de voornaamste oorzaak is van de waargenomen effecten. 
Door de grote gradiënten en het snel schakelen van deze gradiënten worden er extra stromen geïnduceerd in de metalen delen van de MRI scanner. Deze stromen worden eddy currents genoemd en ze veranderen de golfvorm van de gradiënten en daardoor worden vervormingen gevormd in de diffusie-gewogen beelden. De eddy currents kunnen worden verminderd door een zogenaamde double-refocussed spin-echo diffusie gewogen sequentie te gebruiken, maar er is waarschijnlijk nog steeds nabewerking van de beelden nodig om de vervormingen te vinden en ervoor te corrigeren. Het bewegen van een proefpersoon in de scanner is een tweede probleem in de acquisitie van DW-MRI data. Het signaal wordt gevormd op basis van zeer kleine bewegingen van de spins en daarom kunnen zelfs kleine hoofdbewegingen van de proefpersoon het signaal beïnvloeden of zelfs vernietigen. In dit proefschrift presenteren we een iteratieve nabewerkingsmethode om te corrigeren voor zowel beweging als eddy current vervormingen. De methode werd vergeleken met een standaard eddy current en bewegingscorrectie methode en liet een kleine maar waarneembare verbetering zien.

In dit proefschrift presenteren we ook twee toepassingen van DW-MRI. In de eerse studie werd een zeldzaam syndroom, het Landau-Kleffner Syndroom, onderzocht. Na aanvankelijk een normale ontwikkeling verliezen patiënten lijdend aan het syndroom in een zeer korte periode van weken to maanden de mogelijkheid taal te begrijpen en te gebruiken, en soms zelfs de mogelijkheid natuurlijke geluiden te begrijpen. Het syndroom presenteert zichzelf in de leeftijd van 3 tot 7 jaar. Patiënten lijden aan epilepsie, vaak ook gedurende slaap, die weer verdwijnt zodra de patiënt volwassen wordt. Verbale communicatie kan worden hersteld door het gebruik van visuele communicatie zoals pictogrammen, vingerspelling en/of gebarentaal. Er is nog steeds niet veel bekend over de neurale mechanismen van het syndrooom, maar de hypothese is dat er een beschadiging van het auditieve circuit is.

De hier gepresenteerde casus is een 27-jarige vrouw na de epileptische fase. Er werd een functionele MRI studie uitgevoerd om de integratie van geschreven letters en lettergeluiden te onderzoeken. De gevonden reactie van de linkse primaire auditieve cortex op aangeboden lettergeluiden is veel lager dan de reactie in de rechter primaire auditieve cortex. Ook vonden we dat de integratie van geschreven letters en lettergeluiden in de gyrus en sulcus temporalis superior vergelijkbaar zijn met die gevonden in gezonde proefpersonen. Dit resultaat suggereert dat er een beschadiging bestaat in de hogere taalgebieden. De wittestofbanen in de patiënt werden ook onderzocht met DW-MRI. In beide hersenhelften werd de fasciculus arcuatus gevonden, die de primaire auditieve cortex met taalgebieden verbindt. In de linker hersenhelft konden de korte segmenten van de fasciculus arcuatus, die sensorimotorische integratiegebieden en verbale kortetermijngeheugen gebieden met elkaar verbinden niet gereconstrueerd worden, terwijl deze verbindingen consistent gevonden worden in de linkse hersenhelft 
van gezonde proefpersonen. In de rechtse hersenhelft van de patiënt werden de korte segmenten wel teruggevonden, terwijl deze meestal niet kunnen worden gevonden in de meerderheid van gezonde proefpersonen. De resultaten kunnen er daarom op wijzen dat de stoornis in het verbale kortetermijngeheugen dat geconstateerd wordt in Landau-Kleffner patiënten zijn oorsprong vindt in een desintegratie in de wittestofbanen die de taalgebieden met sensorimotorische integratiegebieden en het verbale kortetermijngeheugen.

In de laatste studie gepresenteerd in dit proefschrift onderzoeken we de verbindingen die mogelijk verantwoordelijk zijn voor onbewuste waarnememing van visuele stimuli met een emotionele waarde. In blindsight patiënten, waarbij de visuele cortex is beschadigd door een ongeluk of een infarct, is aangetoond dat ze emotionele visuele stimuli kunnen waarnemen en de hypothese is dat ze daarbij de visuele cortex overslaan en in plaats daarvan een sub-corticaal systeem gebruiken dat bestaat uit de amygdala, pulvinar en superior colliculus. Dit subcorticale systeem werd eerder gevonden in primaten maar nog niet in mensen. Met behulp van op DW-MRI gebaseerde fiber tracking hebben we in een blindsight patiënt en vijf gezonde proefpersonen bewijs gevonden voor het bestaan van een amygdala-pulvinar-superior colliculus verbinding. Interessant daarbij is dat in de blindsight patiënt de verbinding sterker was dan in de gezonde proefpersonen. De resultaten wijzen er mogelijk op dat er sub-corticale plasticiteit en reorganisatie van de witte stof bestaat tussen deze sub-corticale gebieden als gevolg van destructie van de visuele cortex. 